\title{
Compilation of Thermal Properties of Hydrogen in Its Various Isotopic and Ortho-Para Modifications
}

\author{
By Harold W. Woolley, Russell B. Scott, and F. G. Brickwedde
}

New developments in science and industry are aided by accurate knowledge of the behavior of important substances. The great abundance of chemical processes and compounds in which hydrogen is involved make it of particular interest. The experimental and derived data presented here for hydrogen extend over a large range of temperature. Low temperatures are required for the liquid and solid, and moderate and high temperatures occur in chemical reactions.

The available thermal data for $\mathrm{H}_{2}, \mathrm{HD}$, and $\mathrm{D}_{2}$ in solid, liquid, and gaseous states have been brought together, including the distinctive properties of ortho and para forms of $\mathrm{H}_{2}$ and $\mathrm{D}_{2}$. Some data not previously published have been added. The thermal data include thermodynamic functions for the ideal gas state, equilibrium constants, data of state, viscosity, and thermal conductivity with dependence on the pressure, vapor pressure, solid-liquid equilibria, specific heats, and latent heats. Values of state derivatives useful in thermodynamic calculations have been given for normal hydrogen, and the related differences between thermodynamic functions for real and ideal gas states have been evaluated. A temperature entropy diagram for normal $\mathrm{H}_{2}$ in the range of experimental data is also given. The compiled thermal properties of hydrogen are presented in 38 tables, 33 graphs, and numerous equations. The sources of the data have been given in an extensive bibliography.

\section{Introduction}

It was recommended by the National Research Council Committee on Thermal Data for Chemical Industries ${ }^{12}$ and by others that the thermal data on substances of industrial importance should be reexamined with the intention of preparing consistent tables of thermal data of especial interest to chemical engineers and investigators.

In this paper thermal data on hydrogen in its various isotopic and ortho-para modifications are compiled and correlated. Data on properties of the gaseous, liquid, and solid states are presented in tables and graphs, and by use of formulas. Thermodynamic properties are given for the ideal gas state. In addition, tables based on the PVT data for the real gas furnish the additional information required for the calculation of the thermodynamic properties of the real gas. For the con-

\footnotetext{
${ }^{1}$ Division of Chemistry and Chemical Technology, National Research Council.

${ }^{2}$ F. Russell Bichowsky, Chairman, 1938 to 1947.
}

densed phases, directly observable properties are given. Because of the industrial importance of flow and heat-transfer problems, correlations of viscosity and of thermal conductivity are included and their dependence upon pressure discussed briefly. A number of topics are discussed in detail to explain the fundamental principles involved. Most of the data included were taken from published papers. However, a small proportion are based on unpublished measurements made at the Bureau.

The following are the symbols and values of physical constants and conversion factors used in this paper.

\section{Symbols}

Many symbols that are not used extensively in this paper have been omitted from thís list. $A$, constant in an equation for a PV'T isotherm. $B$, second virial coefficient in equation of state of gas.

$B_{v}$, rotational spectroscopic constant. 
$b, b$, constant in an equation for a PVT isotherm; also, a constant in an equation of state.

$C, C$, constant in an equation for a PVT isotherm; also, the Sutherland constant in a viscosity formula.

$C^{\prime}$, constant in an equation for a PVT isotherm.

$C_{p}^{\circ}$, molar heat capacity (molar specific heat) at constant pressure for ideal gas.

$C_{s}$, molar heat capacity (molar specific heat) along a saturation curve.

$C_{v}^{\circ}$, molar heat capacity (molar specific heat) at constant volume for ideal gas.

$c, c$, velocity of light; also a constant in an equation for a PVT isotherm.

$c_{2}$, radiation constant $h c / k$.

$D_{v}$, rotational spectroscopic constant.

$E$, a thermodynamic function, internal energy per mole.

$E^{\circ}, E$ for a substance in the ideal gaseous state.

$E_{0}^{\circ}, E^{\circ}$ at the absolute zero of temperature when for each molecule the energy associated with internal degrees of freedom is at its lowest quantized value.

$F$, a thermodynamic function, molar free energy $F=E+P V-T S$.

$F^{\circ}, F$ for a substance in the ideal gaseous state at

a pressure of 1 atmosphere.

$F_{v}$, rotational spectroscopic constant.

$F_{v, J}$, or $F$, term value.

$f$, a thermodynamic function, fugacity:

$G_{v}$, vibrational term value.

$g$, statistical weight of a quantum level.

$H$, a thermodynamic function, molar heat content or enthalpy, $H=E+P V$.

$H^{\circ}, H$ for a substance in the ideal gaseous state.

$H_{v}$, rotational spectroscopic constant.

$h$, Planch's constant.

$i$, nuclear spin.

$J$, rotational quantum number.

$K$, equilibrium constant.

$k, k$, Boltzmann constant; also, thermal conductivity.

$L_{v}$, latent heat of vaporization.

$M$, molecular weight.

$m$, reduced mass for molecule.

$N$, total number of molecules considered.

$N_{j}$, number of molecules in a given quantum level.

$N_{0}$, Avogadro's number.

$\mathrm{P}$, pressure.

$\mathrm{P}_{c}$, pressure at the critical point.
$P_{0}$, pressure of 1 standard atmosphere, $1.01325 \times$ $10^{6}$ dynes $\mathrm{cm}^{-2}$ by definition.

$p$, momentum corresponding to generalized coordinate $q$.

$q$, a generalized coordinate.

$R$, molar gas constant.

$r$, atomic separation.

$r_{e}$, atomic separation $r$ for minimum potential energy.

$S$, a thermodynamic function, molar entropy.

$S^{\circ}, S$ for a substance in the ideal gaseous state at a pressure of 1 atmosphere.

$T$, absolute temperature on the Kelvin scale.

$T_{c}$, temperature $T$ at critical point.

$T_{0}$, Kelvin temperature $T$ of the ice point, that is, of $0^{\circ} \mathrm{C}$.

$U$, intramolecular potential energy.

$U_{11}$, ratio of mean free path lengths for diffusion and viscosity.

$V$, molar volume.

$V_{c}$, molar volume at the critical point.

$V_{0}$, molar volume of gas at 1-atmosphere pressure and the ice point.

$v_{0}$, molar volume of liquid at zero pressure.

$v$, vibrational quantum number.

$Z$, abbreviation for $P V / R T$.

$\gamma$, ratio of specific heats, $C_{p} / C_{r}$.

$\epsilon$, energy for a quantum state.

$\eta$, viscosity.

$\Theta$, a characteristic Kelvin temperature for a crystal lattice in Debye's theory of specific heats.

$\Lambda$, length of mean free path.

$\mu$, Joule-Thomson coefficient.

$\xi$, fractional increase in atomic separation beyond that for minimum potential energy.

$\rho$, density in Amagat units.

$\sigma$, a correlation function for PVT data.

$\chi$, a function in one equation of state.

$\varphi$, a correlation function for PVT data.

\section{Values Used for Some Physical Constants and Conversion Factors}

Numbers in parentbeses refer to the references given below)

$c$ (velocity of light $=2.99776 \times 10^{10} \mathrm{~cm} \mathrm{sec}^{-1}$ (1).

$c_{2} \quad($ radiation constant $)=\frac{h c}{k}=\frac{N_{0} h c}{R}=1.4384 \mathrm{~cm}$ deg (2).

$h$ (Planck's constant $=6.624 \times 10^{27} \mathrm{~cm} \mathrm{sec} \mathrm{(1).}$

$N_{0}$ (Avogadro number $)=6.0228 \times 10^{23} \mathrm{~mole}^{-1} \cdot(1)$. 
$P_{0}$ (pressure of standard atmosphere $)=1.01325 \times$ $10^{6}$ dynes $\mathrm{cm}^{-2}(3)$

$R$ (molar gas constant $)=N_{0} k=8.3144 \times 10^{7}$ erg mole $^{-1} \operatorname{deg}^{-1}(1)$.

$=1.98714$ thermochemical cal mole ${ }^{-1} \mathrm{deg}^{-1}(4)$.

$T_{0}$ (Kelvin temperature of ice point $)=273.16^{\circ} \mathrm{K}$ (5).

Atomic weight of hydrogen $\left(\mathrm{H}^{1}\right)$ on chemical scale $=1.000786(1)$.

Atomic weight of deuterium $\left(\mathrm{D}\right.$ or $\left.\mathrm{H}^{2}\right)$ on chemical scale $=2.01418$ (1).

1 thermochemical calorie $=4.1833$ international joules (5).

1 international joule $(\mathrm{NBS})=1.000165$ absolute joules (6).

(1) Raymond T. Birge, Rev. Modern Phys. 13, 233 (1941).

(2) Birge's value (Rev. Modern Phys. 13, 233 (1941)) adjusted for later NBS value of the ratio international coulomb/absolute coulomb $=0.99985$; see also reference $(7)$.

(3) Definition.

(4) Birge's value (Rev. Modern Phys. 13, 233 (1941)) adjusted to thermochemical calorie and NBS value for ratio international joule/absolute joule.

(5) Definition.

(6) NBS Technical News Bulletin 31, 49 (1947).

(7) R. W. Curtis, R. L. Driscoll, and C. L. Critchfield, J. Research NBS 28, 133 (1942).

\section{Thermodynamic Properties for the Hydrogens in the Ideal Gas State}

\section{General Principles of Calculation}

For a gas in a state of extreme rarefaction the energy of interaction between molecules forms a minute part of the total energy of the gas. At such low pressures the thermodynamic properties of the gas may be calculated from the spectroscopically determined energies of the single molecules and the general physical constants without considering the energy of interaction of one molecule with another. Some thermodynamic properties, as for example molar entropy and free energy, do not approach a definite value as the pressure of the gas goes to zero. For this reason, values of thermodynamic functions of a gas at low pressure are often indicated by giving values for a pressure of $1 \mathrm{~atm}$ for a fictitious ideal gas having in the limit of low pressure the same thermodynamic functions as the actual gas. The result is then said to be for the gas at a pressure of 1 atmosphere in the hypothetical ideal gas state. Data of state may be used to calculate the differ- ences between properties in the real and ideal gas states.

The procedure for calculating the thermodynamic properties of a substance in the ideal gas state has been discussed by many writers $[3,30$, $31,32] .^{3}$

In outline, it involves the following ideas: The average number $n_{1}$ of molecules in a quantum state of energy $\epsilon_{1}$ is related to the average number, $n_{2}$ of molecules in another state of energy $\epsilon_{2}$ by the Boltzmann distribution law

$$
n_{1} / n_{2}=e^{-\epsilon_{1} / k T} / e^{-\epsilon_{2} / k T}=e^{-\left(\epsilon_{1}-\epsilon_{2}\right) / k T},
$$

where $k$ is the Boltzmann constant, and $T$ is the absolute temperature.

As there are often several states having the same energy, the number of molecules in a given energy level ${ }^{4}$ is also proportional to the number of states, g. If $N_{1}, N_{2}, N_{3}, \ldots$ are the numbers of molecules in the levels $\epsilon_{1}, \epsilon_{2}, \epsilon_{3}, \ldots$., respectively, the number of molecules in any one level is

$$
N_{j}=\frac{N g_{j} e^{-\epsilon_{j} / k T}}{g_{1} e^{-\epsilon_{1} / k T}+g_{2} e^{-\epsilon_{2} / k T}+\ldots .}=\frac{N g_{j} e^{-\epsilon_{j} / k T}}{\sum g_{j} e^{-\epsilon_{j} / k T}},
$$

where $N$, the total number of molecules being considered, is equal to $\Sigma N_{j}$. If properties are to be expressed on the basis of 1 mole, $N$ is taken equal to Avogadro's number, $N_{0}$.

The quantum states are specified by means of quantum numbers, the integer values which certain natural variables have when a molecule has a stationary value of energy. The magnitude of the energy is generally expressed in terms of these numbers. In diatomic molecules, the quantum numbers of interest are $J$, the rotational quantum number, $K$, the rotational quantum number apart from spin, and $v$, the vibrational quantum number. The electronic state is also similarly quantized, and quantum numbers appropriate to it may likewise be assigned. The nuclear spins of the two constituent atoms are designated by $i_{1}$ and $i_{2}$. In terms of these numbers, the statistical weight, $g$, of a level of a diatomic molecule composed of unlike atoms, as for example HD, is $g_{e}\left(2 i_{1}+1\right)\left(2 i_{2}+1\right)(2 J+1)$, where $g_{e}$ is the weight of the electronic level of the mole-

\footnotetext{
${ }^{3}$ Figures in brackets indicate the literature references at the end of this

${ }_{4}^{4}$ The term state is used in the sense that two states differ if any of all the quantum numbers associated with the states are different. The term level is used to express the idea that the energy has a definite value. The statistical weight, $g$, of a level is the number of states having the energy which define the level. A level with more than one state is said to be degenerate.
} paper. 
cule. The ground electronic level of HD, and of $\mathrm{H}_{2}$ and $\mathrm{D}_{2}$, also, is a singlet state, and accordingly $g_{e}$ is 1 .

The proton and deuetron spins are $1 / 2$ and 1 , respectively. For diatomic molecules composed of like atoms, as for example, $\mathrm{H}_{2}$ and $\mathrm{D}_{2}$, there is a division of the rotational levels of the molecule into two groups referred to as the ortho and para series, one of which is composed of the even numbered and the other of the odd numbered rotational levels. Ordinarily, transitions between ortho and para levels are relatively rare, so that the gas can be considered as a mixture of two distinct components. The high temperature equilibrium mixture of the two forms is called the normal mixture, and the more abundant component of the normal mixture is called the ortho component. The statistical weights of the two series depends upon the quantum statistics applicable to the nuclei. For $\mathrm{H}_{2}$ it is the Fermi-Dirac statistics, for $\mathrm{D}_{2}$ the Bose-Einstein statistics.

\section{Fermi-Dirac statistics:}

$$
\begin{aligned}
& g(\text { para series, even } J \text { 's })= \\
& g_{e}(2 i+1) i(2 J+1) \\
& g(\text { ortho series, odd } J \text { 's })= \\
& g_{e}(2 i+1)(i+1)(2 J+1)
\end{aligned}
$$

Bose-Einstein statistics:

$$
\begin{aligned}
& g(\text { ortho series, even } J \text { 's })= \\
& g_{e}(2 i+1)(i+1)(2 J+1) \\
& g\left(\text { para series, odd } J^{\prime} s\right)= \\
& g_{e}(2 i+1) i(2 J+1)
\end{aligned}
$$

The energy per mole due to molecular rotation and intramolecular vibration is

$$
E_{v+r}=\Sigma N_{j} \epsilon_{j}=\frac{N_{0} \Sigma g_{j} \epsilon_{j} e^{-\epsilon_{j} / k T}}{\Sigma g_{j} e^{-\epsilon_{j} / k T}},
$$

where the $\epsilon$ 's are the energies of the rotationalvibrational levels relative to the lowest energy level of the molecule. The translational energy, $3 / 2 N_{0} k T$ or $3 / 2 R T$, is added to this to get $E^{\circ}-E_{0}^{\circ}$, the total internal energy per mole for the ideal gas above the chosen zero in which there would be no translational energy and each mole- cule would be in the lowest energy state available to any form of the molecule. ${ }^{5}$

$$
E^{\circ}-E_{0}^{\circ}=3 / 2 R T+N_{0} \frac{\underset{j}{\sum} g_{j} \epsilon_{j} e^{-\epsilon_{j} / k T}}{\sum g_{j} e^{-\epsilon_{j} / k T}} .
$$

The superscript zero is used to indicate the ideal gas state.

The enthalpy $H^{\circ}$, the specific heats $C_{v}^{\circ}$ and $C_{p}^{\circ}$, the entropy $S^{\circ}$, and the free energy $F^{\circ}$ for the ideal gas state are derivable in accordance with familiar methods of thermodynamics from (1) the internal energy $E^{\circ}-E_{0}^{\circ}$, (2) the equation of state $P V=R T$, and (3) the translational entropy $S_{t}^{\circ}$ of an ideal gas of molecular weight $M$. The equations for these properties as functions of $\left(\epsilon_{j} / k T\right)$ are

$$
\begin{gathered}
\frac{E^{\circ}-E_{0}^{\circ}}{R T}=\frac{\sum_{j} g_{j}\left(\epsilon_{j} / k T\right) e^{-\epsilon_{j} / k T}}{\sum_{j} g_{j} e^{-\epsilon_{j} / k T}}+\frac{3}{2} . \\
\frac{H^{\circ}-E_{0}^{\circ}}{R T}=\frac{E^{\circ}-E_{0}^{\circ}}{R T}+1 .
\end{gathered}
$$

$\frac{C_{v}^{\circ}}{R}=\frac{\sum_{j} g_{j}\left(\epsilon_{j} / k T\right)^{2} e^{-\epsilon_{j} / k T}}{\sum_{j} g_{j} e^{-\epsilon_{j} / k T}}-\left(\frac{\underset{j}{\sum} g_{j}\left(\epsilon_{j} / k T\right) e^{-\epsilon_{j} / k T}}{\underset{j}{\sum} g_{j} e^{-\epsilon_{j} / k T}}\right)^{2}+\frac{3}{2}$.

$$
\frac{C_{p}^{\circ}}{R}=\frac{C_{v}^{\circ}}{R}+1
$$

$$
\begin{gathered}
\frac{S^{\circ}}{R}=\ln \sum_{j} g_{j} e^{-\epsilon_{j} / k T}+\frac{\sum_{j} g_{j}\left(\epsilon_{j} / k T\right) e^{-\epsilon_{j} / k T}}{\sum g_{j} e^{-\epsilon / k T}}+\frac{S_{t}^{\circ}}{R} \cdot(2.11) \\
\frac{S_{t}^{\circ}}{R}=\frac{5}{2} \ln T+3 / 2 \ln M-\ln \left(P / P_{0}\right)+\ln \frac{(2 \pi)^{3 / 2} R^{5 / 2}}{h^{3} N_{0}^{4} P_{0}}+\frac{5}{2} .
\end{gathered}
$$

$$
\frac{S_{t}^{\circ}}{R}=\frac{3}{2} \ln T+3 / 2 \ln M+\ln V+\ln \frac{(2 \pi R)^{3 / 2}}{h^{3} N_{0}^{4}}+\frac{5}{2}
$$

$\frac{F^{\circ}-E_{0}^{\circ}}{R T}=\frac{H^{\circ}-E_{0}^{\circ}-T S^{\circ}}{R T}=-\ln \Sigma_{j} g_{j} e^{-\epsilon_{j} / k T}+\frac{5}{2}-\frac{S_{t}^{\circ}}{R}$.

\footnotetext{
5 Accordingly for orthohydrogen and paradeuterium $E_{0}^{\circ}$ is not the internal energy at $0^{\circ} \mathrm{K}$. For these substances at $0^{\circ} \mathrm{K}$ the internal energy above the chosen zero $(J=0, v=0)$ is the rotational energy per mole of molecules in the rotational level $J=1$. At $0^{\circ} \mathrm{K}$ internal energies of normal hydrogen and normal deuterium are respectively three-fourths the internal energy of orthohydrogen and one-third the internal energy of paradeuterium.
} 
In eq 2.12, $P$ and $P_{0}$ are the pressure of the gas and standard atmospheric pressure, respectively, with both expressed in dynes $\mathrm{cm}^{-2}$. The ratio $P / P_{0}$ is the pressure expressed in atmospheres.

For a monatomic gas in which the ground state is so far below the others in energy that it alone makes appreciable contribution to the state-sum, $\sum_{j} g_{j} e^{-\epsilon_{j} / k T}$, eq 2.7 to 2.14 are simplified considerably. With $\epsilon_{1}$, the energy of the ground state, taken as zero, the state-sum reduces to the constant $g_{1}$

As a result, $\left(E^{\circ}-E_{0}^{\circ}\right) / R T=3 / 2 ;\left(H^{\circ}-E_{0}^{\circ}\right) / R T=$ $5 / 2 ; \quad C_{v}^{\circ} / R=3 / 2 ; \quad C_{p}^{\circ} / R=5 / 2 ; S^{\circ} / R=\ln g_{1}+S_{t}^{\circ} / R$, and $\left(F^{\circ}-E_{0}^{\circ}\right) / R T=-\ln g_{1}+5 / 2-S_{t}^{\circ} / R$. When the nuclear spin is included, $g_{1}$ contains $(2 i+1)$ as a factor.

Normal hydrogen is a mixture 75 percent of orthohydrogen and 25 percent of parahydrogen, and normal deuterium $66 \frac{2}{3}$ percent of orthodeuterium, and $33 \frac{1 / 3}{3}$ percent of paradeuterium. The molar entropy and free energy of a mixture of ideal gases present in the mole fractions $x_{1}, x_{2}$, are

$$
\begin{aligned}
& S_{\text {mixture }}=\sum_{j} x_{j} S_{j}^{\circ}-R \sum_{j} x_{j} \ln x_{j} \\
& F_{\text {mixture }}=\sum_{j} x_{j} F^{\circ}{ }_{j}+R T \underset{j}{\sum} x_{j} \ln x_{j},
\end{aligned}
$$

where $S^{\circ}{ }_{j}$ and $F^{\circ}{ }_{j}$, the molar entropy and free energy of the ideal gas $j$ in a pure state at the pressure of the mixture, are given by eq 2.11 and 2.14, using eq 2.12 for the evaluation of $S^{\circ}{ }_{t}$. The summation $-R \Sigma x_{j} \ln x_{j}$ is called the entropy of mixing. Using eq 2.13 for the evaluation of $S_{t}$, and setting $V$ equal to the molar volume of the constituent, that is, the volume of the mixture divided by the moles of constituent present, is equivalent to using partial pressures in eq 2.12, in which case the entropy and free energy of the mixture are equal simply to $\Sigma x_{j} S^{\circ}{ }_{j}$ and $\Sigma x_{j} F^{\circ}{ }_{j}$.

\section{Energy Values From Spectroscopic Data}

The values of $\epsilon_{j}$ to be used in evaluating the equations of the preceding section are derived from analysis of molecular spectra. In general, banded electronic absorption and emission spectra, infrared, rotation-vibration absorption spectra, and Raman spectra are considered. But as the $\mathrm{H}_{2}$ and $\mathrm{D}_{2}$ molecules have no electric dipole moments in their normal states, they have no rotation-vibration absorption spectra. Similarly, no such spectra have been observed for HD, although lack of symmetry permits it to have a very weak dipole moment.

The spectroscopic energy level data for hydrogen are represented by a series in which the energies of the levels relative to the ground level, $v=0$, $J=0$, divided by $h c$ are expressed as a function of the rotational and vibrational quantum numbers $J$ and $v$, see eq 2.17. The quantity $\epsilon_{j} / h c$ is called the term value of the level and is designated by the symbol $F$. Term values are determined experimentally from differences between the wave numbers of spectrum lines and are expressed in terms of reciprocal centimeters as a unit. Here $F_{v, J}$ is the term value for the level $v, J ; F_{0,0}$ for the ground state being zero.

Up to $25,000 \mathrm{~cm}^{-1}$, the term values on which tables 4,7 , and 8 are based, can be represented by

$$
\begin{gathered}
F_{v, J}=G_{v}-G_{0}+B_{v} J(J+1)+D_{v} J^{2}(J+1)^{2}+ \\
F_{v} J^{3}(J+1)^{3}+H_{v} J^{4}(J+1)^{4}+ \\
F_{v} J^{3}(J+1)^{3}-H_{v} J^{4}(J+1)^{4}
\end{gathered}
$$

where the subscripts used indicate the quantum numbers on which the different symbols depend for their values.

The functions $G_{v}, B_{v}, D_{v}, F_{v}$, and $H_{v}$ for $\mathrm{H}_{2}, \mathrm{HD}$ and $\mathrm{D}_{2}$ are as follows:

For $\mathrm{H}_{2}$ :

$$
\begin{aligned}
& G_{v}=4405.3\left(v+\frac{1}{2}\right)-125.325\left(v+\frac{1}{1}\right)^{2}+1.9473\left(v+\frac{1}{2}\right)^{3}-0.11265\left(v+\frac{1}{1 / 2}\right)^{4} \\
& B_{v}=60.8483-3.06635(v+1 / 2)+0.068361\left(v+\frac{1}{2}\right)^{2}-0.0065\left(v+\frac{1}{2}\right)^{3} \\
& D_{v}=-0.046435+0.0014904(v+1 / 2)-0.000063648\left(v+\frac{1}{1}\right)^{2} \\
& F_{v}=4.93203 \times 10^{-5}+0.02800 \times 10^{-5}(v+1 / 2) \\
& H_{v}=-6.7217 \times 10^{-8}
\end{aligned}
$$


For HD:

$$
\begin{aligned}
& G_{v}=3817.09(v+1 / 2)-94.958(v+1 / 2)^{2}+1.4569\left(v+\frac{1}{1 / 2}\right)^{3}-0.07665(v+1 / 2)^{2} \\
& E_{v}=45.6549-1.992721(v+1 / 2)+0.038482(v+1 / 2)^{2}-0.00316885(v+1 / 2)^{\circ} \\
& D_{v}=-0.026136+0.00072661(v+1 / 2)-0.0000268773\left(v+\frac{1}{2}\right)^{2} \\
& F_{v}=2.0827 \times 10^{-5}+0.01024 \times 10^{-5}(v+1 / 2) \\
& H_{v}=-2.1295 \times 10^{-8}
\end{aligned}
$$

For $\mathrm{D}_{2}$ :

$$
\begin{aligned}
& G_{v}=3118.46\left(v+\frac{1}{2}\right)-64.10\left(v+\frac{1}{2}\right)^{2}+1.2514\left(v+\frac{1}{2}\right)^{3}-0.10612(v+1 / 2)^{4}+0.00034\left(v+\frac{1}{2}\right)^{5} \\
& E_{v}=30.4286-1.04917(v+1 / 2)+0.0057934\left(v+\frac{1}{2}\right)^{2}-0.00027486(v+1 / 2)^{3} \\
& D_{v}=-0.011586+0.000151(v+1 / 2)+0.000058\left(v+\frac{1}{1}\right)^{2} \\
& F_{v}=6.22 \times 10^{-6}+0.105 \times 10^{-6}\left(v+\frac{1}{1}\right) \\
& H_{r}=-0.442 \times 10^{-8}
\end{aligned}
$$

The numerical values of the coefficients in eq 2.18 to 2.20 are based on the latest available spectroscopic measurements due principally to Rasetti [2], Hyman [5, 6], Jeppesen [6, 7, 12, 15, 24], Beutler [20, 21], and Teal and Mac Wood [22]. The data of Fujioka and Wada [23] were not used and the data of Mie [16] on HD only through its influence on the formula for $G_{v}$. The equations $G_{v}$ for $\mathrm{H}_{2}$ and $\mathrm{HD}$ are those given by Teal and Mac Wood [22], and that for $\mathrm{D}_{2}$ by Jeppesen [24]. The equations for $B_{v}$ are essentially Jeppesen's $[12,24]$ equations expressed for use with $J(J+1)$. The constants in the equations for $D_{v}, F_{v}$, and $H_{v}$ were obtained from theory using the equations for $G_{v}$ and $B_{v}$ and the formulas of Dunham [10] without his correction terms.

In the case of hydrogen as for many other substances, extrapolations of spectroscopic formulas have to be made into regions of large rotational quantum numbers for which no wavelength measurements are available in order to obtain values for the energies $\epsilon_{j}$ of the higher quantum states. The energy values for large rotational and vibrational quantum numbers are influenced by the law of internuclear force of the molecule for large separations of the nuclei. Special consideration has been given to this point in the present work and two methods were developed whereby more reliable values of the energies of the unobserved higher rotational levels were obtained.

The first improvement was the addition of the final term in eq $2.17,\left[H_{r} J^{4}(J+1)^{4}\right]^{2} /\left[\left(F_{\imath} J^{3}(J+1)^{3}-\right.\right.$ $\left.\left.H_{v} J^{4}(J+1)^{4}\right)\right]$. Without the final term, eq 2.17 is of the form in which spectroscopic data have heretofore been represented, but in that form it is not a good approximation for large values of $J$. The third, fourth, fifth, and sixth terms of eq 2.17 are of alternate sign and for $\mathrm{H}_{2}$ the third, fourth, and fifth terms are approximately equal for $J=28$. This suggested that the series be extended with successive terms in constant ratio. The final term of eq 2.17 is the sum of the geometric series of added terms in which the term to term ratio is that between the fifth and sixth terms of eq 2.17.

This change in the formula for the energies of the rotational-vibrational levels of the normal $\left(1 s^{1} \Sigma\right)$ electronic state of hydrogen has only a small effect on the energy values of the observed spectrum lines. Thus the mean difference between Jeppesen's [12] observed and calculated term values for the $2 p^{1} \Sigma-1 s^{1} \Sigma$ band for $\mathrm{H}_{2}$ was $1.032 \mathrm{~cm}^{-1}$, whereas using eq 2.17 in place of Jeppesen's equation for the $1 s^{1} \Sigma$ state the mean difference between observed and calculated values is $1.030 \mathrm{~cm}^{-1}$.

As a second improvement, for the calculation of thermodynamic properties above $2,000^{\circ} \mathrm{K}$, an alternative determination of the highest rotational levels was made. Instead of using the power series eq 2.17, the energies corresponding to any degree of rotation and vibration were determined from the potential energy. This was 
carried out in effect by (1) determining the potential energy $U$ of the nonrotating $\mathrm{H}_{2}$ molecule as a function of the internuclear separation, (2) adding the rotational energy $h^{2} J(J+1) / 8 \pi^{2} I_{e}\left(r / r_{e}\right)^{2}$ to $U$ to obtain an effective potential energy, $U^{\prime}$, for a molecule with rotational quantum number $J$, and (3) using the quantum condition $\oint p d q=\int(2 m)^{1 / 2}$ $\left(\boldsymbol{\epsilon}_{v, J}-U^{\prime}\right)^{1 / 2} d r=(v+1 / 2) h_{\iota}$ to determine the energy $\boldsymbol{\epsilon}_{v, J}$ of the quantum state $v, J$.

The coefficients of a power series used to represent the molecular potential energy were evaluated for the $\mathrm{H}_{2}$ molecule using Dunham's [10] theoretical relations and the rotational and vibrational data for $\mathrm{H}_{2}$ :

$U=79734 \xi^{2}\left(1-1.6082 \xi+1.8598 \xi^{2}-1.8882 \xi^{3}+\right.$

$$
\left.1.7118 \xi^{4}-1.450 \xi^{5}+1.421 \xi^{6}\right),
$$

where $\xi$ is $\left(r-r_{\epsilon}\right) / r_{e}, r_{e}$ being the equilibrium value of the internuclear separation, and $U$ is expressed in reciprocal centimeters. Although this series is a poor representation of $U$ for internuclear separations twice the equilibrium value (i. e., at $\xi=1$ ), it is very good for small values of $\xi$. Therefore, this series was not used for the potential energy function finally accepted for internuclear separations much greater than the equilibrium value, but it was used for internuclear separations less than the equilibrium value. At dissociation the minimum value of $r$ for classical motion is more than half of $r_{e}$ (i. e., $|\xi|<0.5$ ), and the series determines the inner portion of the potential energy curve with sufficient reliability for the present purposes.

The ranges of internuclear oscillation, $\xi_{\max }-\xi_{\min }$, for different values of the energy needed to fix the outer portion of the potential energy curve, were determined from (1) the vibrational levels of the nonrotating molecule, symbolized by $G_{v}$ in eq 2.17 to eq 2.20 , which have been accurately measured to within $140 \mathrm{~cm}^{-1}$ of dissociation $[5,12,20,21]$ and (2) the quantum condition.

$$
\oint p d q=\left(2 m r_{e}^{2} h c\right)^{1 / 2} \oint\left(G_{v}-U\right)^{1 / 2} d \xi=(v+1 / 2) h .
$$

The method used to obtain $\left(\xi_{\max }-\xi_{\min }\right)$ by satisfying eq 2.22 was essentially that of Rydberg [8] and Klein [9]. Calculated values of the potential energy $U$ in wave numbers are given in table 1 .

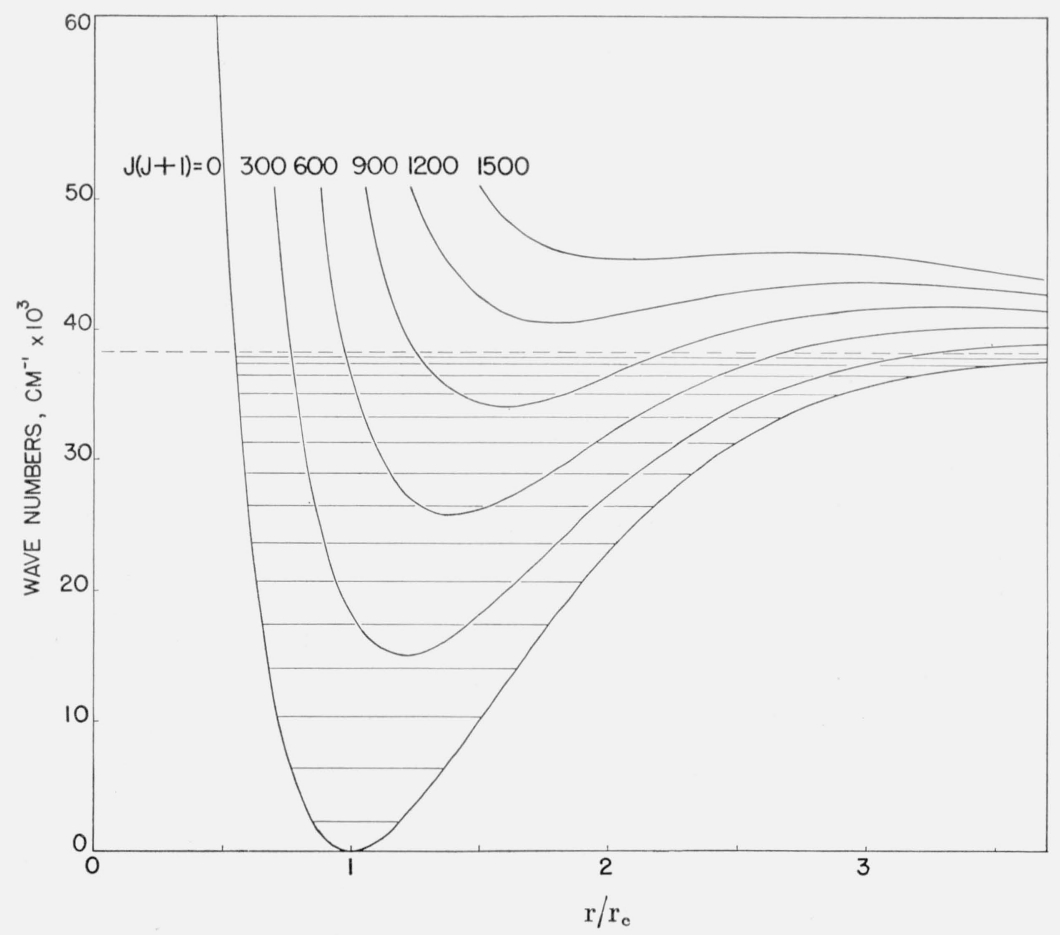

Figure 1. Potential-energy curves for $\mathrm{H}_{2}$. 
TABLE 1. Molecular potential energy $U$ for $\mathrm{H}_{2}$ as a function of $\xi=\left(r-r_{e}\right) / r_{e}$, the change in internuclear separation

\begin{tabular}{|c|c|c|c|c|c|}
\hline$\xi$ & $U$ & $\xi$ & $U$ & $\xi$ & $U$ \\
\hline & $c m-1$ & & $c m-1$ & & $\mathrm{cm-1}$ \\
\hline-0.5 & 53,648 & 0.9 & 20,540 & 2. 3 & 36,828 \\
\hline-.4 & 27,150 & 1.0 & 22,822 & 2. 4 & 37,100 \\
\hline-.3 & 12,338 & 1.1 & 24,915 & 2. 5 & 37,322 \\
\hline-.2 & 4,511 & 1. 2 & 26,810 & 2. 6 & 37,503 \\
\hline-.1 & 942 & 1.3 & 28,505 & 2.7 & 37,650 \\
\hline 0 & 0 & 1.4 & 30,009 & 2. 8 & 37,770 \\
\hline .1 & 683 & 1.5 & 31,329 & 2. 9 & 37,867 \\
\hline .2 & 2,360 & 1.6 & 32,472 & 3.0 & 37,946 \\
\hline .3 & 4,628 & 1.7 & 33,454 & 3.1 & 38,009 \\
\hline .4 & 7,223 & 1.8 & 34,292 & 3. 2 & 38,061 \\
\hline .5 & 9,968 & 1.9 & 35,001 & 3.3 & 38,102 \\
\hline .6 & 12,744 & 2. 0 & 35,599 & 3.4 & 38,136 \\
\hline .7 & 15,466 & 2. 1 & 36,092 & 3.5 & 38,163 \\
\hline .8 & 18,079 & 2. 2 & 36,496 & $\infty$ & 38,296 \\
\hline \multicolumn{6}{|c|}{$r_{e}=0.7414 \times 10^{-8} \mathrm{~cm}$} \\
\hline
\end{tabular}

The effective potential energy curves for rotating molecules obtained by adding to $U$ for the nonrotating molecule the energy of rotation, $J(J+1) B_{e} /(1+\xi)^{2}$, in $\mathrm{cm}^{-1}$, are illustrated in figure 1. By applying the quantum integral,

$\oint p d q=\left(2 m r_{e}^{2} h c\right)^{1 / 2} \oint\left(F-U^{\prime}\right)^{1 / 2} d \xi=(v+1 / 2) h$,

to the effective potential energy curves, $U^{\prime}$, a set of corresponding values of energy $(F)$ and vibrational quantum number was determined for each

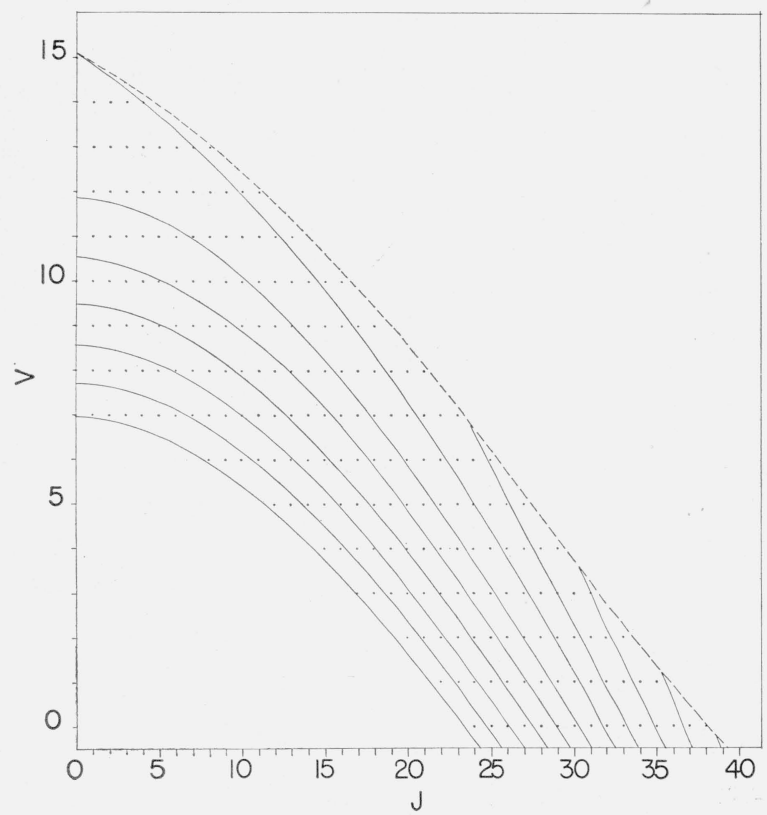

Figure 2. Energy contour diagram for $\mathrm{H}_{2}$. of a few large values of the rotational quantum number. In table 2 these corresponding values are given together with the maximum and minimum values of the energy $(F)$ for different values of $J(J+1)$. The data were used to determine the constant energy lines in the $v$ versus $J$ diagram in figure 2.

TABLE 2. Corresponding values of $v, J(J+1)$, and $F$ obtained by evaluating $\oint p d q=(v+1 / 2) h$

[The values of $v$ and $J$ are not integral and so do not represent stationary states, yet the table values indicate how $F$ depends on $v$ and $J$ over a range including many stationary states.]

\begin{tabular}{|c|c|c|}
\hline $\begin{array}{c}F \\
\text { (above } U \\
\text { at } \xi=0 \text { ) }\end{array}$ & $J(J+1)$ & $v$ \\
\hline $\mathrm{cm}^{-1}$ & & \\
\hline 38,269 & 300 & 8. 8483 \\
\hline 34,269 & 300 & 6. 2874 \\
\hline 30,269 & 300 & 4. 5015 \\
\hline 26,269 & 300 & 2. 9881 \\
\hline 22,269 & 300 & 1. 6461 \\
\hline 38,269 & 600 & 4. 8378 \\
\hline 34,269 & 600 & 2. 7292 \\
\hline 30,269 & 600 & 1. 0757 \\
\hline 38,269 & 900 & 1. 4032 \\
\hline 42,269 & 1,200 & 0.4845 \\
\hline \multicolumn{3}{|c|}{$\begin{array}{l}\text { Maximum values of } F \text { and } v \text { for } \\
\text { given values of } J(J+1)\end{array}$} \\
\hline 38,288 & 0 & 15.053 \\
\hline 39,098 & 300 & 9.919 \\
\hline 40,323 & 600 & 6.615 \\
\hline 41,858 & 900 & 3.929 \\
\hline 43,712 & 1,200 & 1. 703 \\
\hline 45,989 & 1,500 & -0.072 \\
\hline \multicolumn{3}{|c|}{$\begin{array}{c}\text { Minimum values of } F \text { and } v \text { for } \\
\text { given values of } J(J+1)\end{array}$} \\
\hline 0 & 0 & -0.5 \\
\hline 15,027 & 300 & -.5 \\
\hline 25,847 & 600 & -.5 \\
\hline 34,111 & 900 & -.5 \\
\hline 40,606 & 1,200 & -.5 \\
\hline 45,601 & 1,500 & -.5 \\
\hline
\end{tabular}

Table 3 shows that over a wide range of $J$ values the results of the numerical integration just described are in good agreement with the rotational energy formula (eq 2.17) when the last term, corresponding to a geometric series continuation, is included. For the larger values of $J$ there are appreciable differences; yet, when it 
is observed how large the final term of eq 2.17 is in these cases, it seems surprising that the discrepancies between $F$ (table 2) and $F$ (eq 2.17 are as small as they are. In another publication [27] a more rapidly converging series representing $J(J+1)$ as a function of the rotational energy has been suggested.

TABLE 3. Comparison of rotational-vibrational energies $F$ from table 2 and from equation 2.17

\begin{tabular}{|c|c|c|c|c|}
\hline$J(J+1)$ & $v$ & $\begin{array}{c}F \text { (table } \\
2 \text { ) }\end{array}$ & $\begin{array}{c}F \text { (table } \\
2)-F \\
(\mathrm{eq} 2.17)\end{array}$ & $\begin{array}{c}\text { Final term } \\
\text { of eq } 2.17\end{array}$ \\
\hline & & $\mathrm{cm}-1$ & $c m^{-1}$ & $c m^{-1}$ \\
\hline 300 & 4. 5015 & 30,269 & -54 & 155 \\
\hline 300 & 6. 2874 & 34,269 & -34 & 154 \\
\hline 600 & 1. 0757 & 30,269 & -78 & 3,904 \\
\hline 900 & 1. 4032 & 38,269 & -300 & 24,192 \\
\hline 1,200 & 0.4845 & 42,269 & 761 & 86,345 \\
\hline
\end{tabular}

\section{Details of the Calculations and Results}

In the evaluation of the series of section II, 1 for the calculation of the thermal properties, direct summation was employed for temperatures below $2,000^{\circ} \mathrm{K}$. The resulting values to $2,000^{\circ}$ $\mathrm{K}$ for the various thermodynamic functions $S^{\circ}$, $H^{\circ}-E_{0}^{\circ}, \quad-\left(F^{\circ}-E_{0}^{\circ}\right) / T$, and $C_{p}^{\circ}$ for the ideal gas state at one atmosphere pressure are tabulated in tables 4,5 , and 6 , for $\mathrm{H}_{2}, \mathrm{HD}$, and $\mathrm{D}_{2}$. For $n-\mathrm{H}_{2}$ for temperatures above $2,000^{\circ} \mathrm{K}$, the contributions due to levels below $25,000 \mathrm{~cm}^{-1}$ were calculated by direct summation, whereas for levels above $25,000 \mathrm{~cm}^{-1}$ a less laborious method was used involving the determination of the number of levels within successive equal steps of $2,000 \mathrm{~cm}^{-1}$ in the rotational vibrational energy, using the results of the calculations of the last section which led to figures 1 and 2. For these

TABLE 4. Thermodynamic functions for $\mathrm{H}_{2}$ in the ideal gaseous state

Values for $S^{\circ}$ and $-\left(F^{\circ}-E_{0}^{\circ}\right) / T$ include nuclear spin

\begin{tabular}{|c|c|c|c|c|c|c|c|c|c|c|c|c|}
\hline \multirow{2}{*}{ Temperature } & \multicolumn{3}{|c|}{$S^{\circ}$, cal mole ${ }^{-1} \mathrm{deg}-1$} & \multicolumn{3}{|c|}{$H^{\circ}-E_{0}^{\circ}$, cal mole -1} & \multicolumn{3}{|c|}{$-\frac{F^{\circ}-F_{0}^{\circ}}{T}$, cal mole -1 deg -1} & \multicolumn{3}{|c|}{$C_{p}^{\circ}$ cal mole ${ }^{-1}$ deg -1} \\
\hline & $p-\mathrm{H}_{2}$ & $0-\mathrm{H}_{2}$ & $n-\mathrm{H}_{2}$ & $p-\mathrm{H}_{2}$ & $0-\mathrm{H}_{2}$ & $n-\mathrm{H}_{2}$ & $p-\mathrm{H}_{2}$ & $0-\mathrm{H}_{2}$ & $n-\mathrm{H}_{2}$ & $p-\mathrm{H}_{2}$ & $0-\mathrm{H}_{2}$ & $n-\mathrm{H}_{2}$ \\
\hline${ }^{\circ} K$ & & & & & & & & & & & & \\
\hline $10 \ldots \ldots$ & 11. 215 & 15. 581 & 15. 607 & 49.6785 & 388.327 & 303.665 & 6.247 & -23.252 & -14.760 & 4. 968 & 4. 968 & 4. 968 \\
\hline $20 \ldots$ & 14. 658 & 19. 024 & 19. 050 & 99.357 & 438.006 & 353.344 & 9.690 & -2.876 & 1. 382 & 4. 968 & 4. 968 & 4. 968 \\
\hline 20.39 & 14. 754 & 19. 120 & 19. 146 & 101.295 & 439. 943 & 355.281 & 9. 786 & -2.457 & 1. 721 & 4. 968 & 4. 968 & 4. 968 \\
\hline $30 \ldots$ & 16. 672 & 21. 039 & 21. 064 & 149. 036 & 487.684 & 403.022 & 11. 705 & 4. 783 & 7. 630 & 4. 968 & 4. 968 & 4. 968 \\
\hline $33.1 \ldots$ & 17. 161 & 21.527 & 21.553 & 164. 437 & 503.085 & 418.423 & 12.193 & 6.328 & 8. 911 & 4. 968 & 4. 968 & 4. 968 \\
\hline $40 \ldots$ & 18. 102 & 22.468 & 22. 494 & 198. 729 & 537.363 & 452. 705 & 13. 134 & 9.034 & 11. 176 & 4. 973 & 4. 968 & 4. 969 \\
\hline $50 \ldots$ & 19. 214 & 23.576 & 23. 603 & 248.581 & 587.041 & 502.426 & 14. 243 & 11.836 & 13.554 & 5.007 & 4. 968 & 4. 978 \\
\hline $60 \ldots$ & 20.135 & 24.492 & 24.513 & 299. 106 & 636.722 & 552.318 & 15.150 & 13.870 & 15. 307 & 5.115 & 4. 969 & 5.005 \\
\hline $70 \ldots$ & 20. 938 & 25. 248 & 25. 288 & 351.222 & 686.422 & 602.622 & 15. 921 & 15. 442 & 16. 679 & 5. 330 & 4. 972 & 5. 061 \\
\hline $80 \ldots$ & 21.669 & 25. 913 & 25. 969 & 406. 015 & 736.179 & 653.638 & 16. 594 & 16. 710 & 17. 799 & 5. 646 & 5. 982 & 5. 148 \\
\hline $90 \ldots$ & 22. 356 & 26.500 & 26.581 & 464.385 & 786.085 & 705.660 & 17.197 & 17. 766 & 18. 741 & 6. 036 & 5. 003 & 5. 261 \\
\hline $100 \ldots$ & 23. 014 & 27.029 & 27.142 & 526.837 & 836.277 & 758. 916 & 17. 745 & 18.667 & 19.554 & 6.455 & 5. 039 & 5. 393 \\
\hline $120 \ldots$ & 24. 259 & 27. 959 & 28.151 & 663.752 & 938. 227 & 869. 609 & 18. 729 & 20.140 & 20.904 & 7. 204 & 5. 170 & 5. 678 \\
\hline $150 \ldots$ & 25.945 & 29.143 & 29. 461 & 890.605 & $1,097.78$ & $1,045.99$ & 20.007 & 21.825 & 22. 488 & 7.807 & 5. 487 & 6.067 \\
\hline $200 \ldots$ & 28. 202 & 30.808 & 31. 275 & $1,282.70$ & $1,387.90$ & $1,361.61$ & 21. 788 & 23.869 & 24.466 & 7. 742 & 6.110 & 6. 518 \\
\hline $250 \ldots$ & 29. 889 & 32.225 & 32. 758 & $1,660.49$ & $1,705.80$ & $1,694.47$ & 23. 246 & 25. 402 & 25.981 & 7. 380 & 6. 565 & 6. 770 \\
\hline $298.16 \ldots$ & 31.168 & 33.404 & 33. 963 & $2,009.99$ & $2,028.34$ & $2,023.75$ & 24.426 & 26. 602 & 27.175 & 7. 158 & 6. 803 & 6. 891 \\
\hline 300 & 31.212 & 33.446 & 34.005 & $2,023.16$ & $2,040.87$ & $2,036.44$ & 24.468 & 26.643 & 27. 217 & 7. 152 & 6. 809 & 6. 894 \\
\hline $350 \ldots$ & 32.306 & 34.505 & 35.073 & $2,377.84$ & $2,384.39$ & $2,382.75$ & 25. 512 & 27.693 & 28. 265 & 7.049 & 6.917 & 6. 951 \\
\hline $400 \ldots$ & 33.244 & 35.432 & 36.003 & $2,729.19$ & $2,731.54$ & $2,730.95$ & 26.421 & 28.603 & 29.175 & 7. 010 & 6. 963 & 6. 975 \\
\hline 500 & 34.806 & 36. 990 & 37.561 & $3,429.24$ & $3,429.53$ & $3,429.46$ & 27. 948 & 30.131 & 30. 702 & 6. 998 & 6. 992 & 6. 993 \\
\hline 600 & 36.083 & 38. 266 & 38.838 & $4,129.48$ & $4,129.52$ & $4,129.51$ & 29. 200 & 31.383 & 31.955 & 7. 010 & 7. 009 & 7. 009 \\
\hline 700 & 37.165 & 39.348 & 39.920 & $4,831.65$ & $4,831.66$ & $4,831.66$ & 30.263 & 32.446 & 33.018 & 7. 037 & 7. 036 & 7. 036 \\
\hline $1,000 \ldots$ & 39. 701 & 41.884 & 42.455 & & & $6,966.23$ & 32.735 & 34.918 & 35.490 & $\ldots$ & & 7. 219 \\
\hline $1,500 \ldots$ & 42. 720 & 44. 903 & 45.475 & - & - & $10,697.20$ & 35. 589 & 37. 770 & 38. 343 & ..... & & 7. 720 \\
\hline $2,000 \ldots$ & 45.007 & 47.190 & 47. 762 & -...- & -......... & $14,679.2$ & 37.668 & 39.851 & 40.422 & $\ldots$ & & 8. 195 \\
\hline $3,000 \ldots$ & -...... & -..... & 51. 221 & ....... & .......... & $23,230.9$ & -..... & -...... & 43.478 & $\ldots$ & & 8. 859 \\
\hline $4,000 \ldots$ & - n...- & - n- & 53. 839 & $\ldots$ & - n. & 32,345 & -.... & - & 45. 753 & $\ldots$ & & 9. 342 \\
\hline $5,000 \ldots$ & -..... & -..... & 55. 969 & -..... & - & 41,895 . & -..... & - & 47. 590 & $\ldots$ & & 9. 748 \\
\hline
\end{tabular}


TABLE 5. Thermodynamic functions for $H D$ in the ideal gas state

Values for $S^{\circ}$ and $-\left(F^{\circ}-E_{0}^{\circ}\right) / T$ include nuclear spin

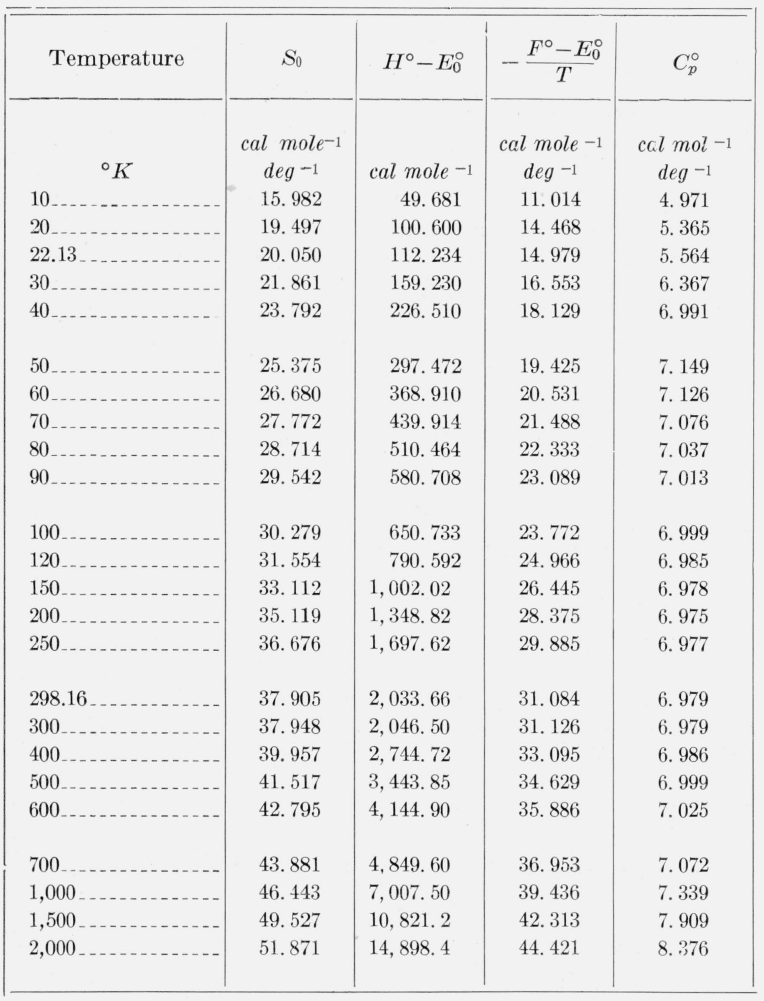

higher levels having characteristic temperatures above $36,000^{\circ} \mathrm{K}$, the exact placement of each individual level is not important for calculations up to $5,000^{\circ} \mathrm{K}$.

Figure 1 shows that the effective potential energy curves for rotational quantum numbers other than 0 have broad potential energy barriers above the minimum dissociation energy, 38,296 $\mathrm{cm}^{-1}$, for $J=0$. As a result there are above 38,296 $\mathrm{cm}^{-1}$, the minimum dissociation energy, quantized rotational-vibrational levels belonging to the sequences of levels below $38,296 \mathrm{~cm}^{-1}$. These states are represented by the points in figure 2 between the dashed curve and the full line dissociation energy curve passing through $(J=0$, $v=15.1)$ and $(J=32.5, v=-1 / 2)$.

It seemed proper to include in the calculations of the thermal properties of hydrogen above $2,000^{\circ}$ $\mathrm{K}$ these quantized or partially quantized rotational-vibrational states. The values of the thermodynamic functions for $n-\mathrm{H}_{2}$ from $2,000^{\circ}$ to $5,000^{\circ} \mathrm{K}$ in table 4 are based on this convention.

The effect of the quantized rotational-vibrational levels above the minimum dissociation energy of $\mathrm{H}_{2}$ on the most sensitive of the functions calculated, namely the molecular heat capacity, is represented in figure 3. Curve $A$ represents the

TABLE 6. Thermodynamic functions for $\mathrm{D}_{2}$ in the ideal gaseous state

Values for $S^{\circ}$ and $-\left(F^{\circ}-E_{0}^{\circ}\right) / T$ include nuclear spin

\begin{tabular}{|c|c|c|c|c|c|c|c|c|c|c|c|c|}
\hline \multirow{2}{*}{ Temperature } & \multicolumn{3}{|c|}{$S^{\circ}$, cal mole-1 deg -1} & \multicolumn{3}{|c|}{$H^{\circ}-E_{0}^{\circ}$, cal mole ${ }^{-1}$} & \multicolumn{3}{|c|}{$-\frac{F^{\circ}-F_{0}^{\circ}}{T}$, cal mole-1 $\operatorname{deg}^{-1}$} & \multicolumn{3}{|c|}{$C_{p}^{\circ}$, cal mole-1 $\mathrm{deg}^{-1}$} \\
\hline & $p-\mathrm{D}_{2}$ & $0-\mathrm{D}_{2}$ & $n-\mathrm{D}_{2}$ & $p-\mathrm{D}_{2}$ & $0-\mathrm{D}_{2}$ & $n-\mathrm{D}_{2}$ & $p-\mathrm{D}_{2}$ & $0-\mathrm{D}_{2}$ & $n-\mathrm{D}_{2}$ & $p-\mathrm{D}_{2}$ & $0-\mathrm{D}_{2}$ & $n \cdot \mathrm{I}_{2}$ \\
\hline${ }^{\circ} K$ & & & & & & & & & & & & \\
\hline 10 & 17.645 & 16.839 & 18. 372 & 220.505 & 49.679 & 106.621 & -4.406 & 11.871 & 7.710 & 4. 968 & 4. 968 & 4. 968 \\
\hline $20 \ldots$ & 21.088 & 20. 283 & 21.816 & 270.183 & 99.364 & 156.303 & 7.579 & 15.315 & 14. 001 & 4. 988 & 4. 972 & 4. 971 \\
\hline $23.57 \ldots$ & 21.904 & 21.101 & 22. 633 & 287.918 & 117.139 & 174.065 & 9. 689 & 16. 131 & 15. 248 & 4. 968 & 4. 989 & 4. 982 \\
\hline $30 \ldots$ & 23. 102 & 22. 315 & 23.842 & 319.863 & 149.514 & 206. 297 & 12. 440 & 17.331 & 16. 965 & 4. 968 & 5.105 & 5.059 \\
\hline 40 & 24. 533 & 23.843 & 25. 338 & 369.584 & 202.775 & 258.378 & 15. 293 & 18. 774 & 18.879 & 4. 980 & 5. 617 & 5. 404 \\
\hline $50 \ldots$ & 25. 649 & 25. 180 & 26. 600 & 419.599 & 262.811 & 315.048 & 17. 257 & 19. 923 & 20.299 & 5.033 & 6.412 & 5. 952 \\
\hline $60 \ldots$ & 26.576 & 26.418 & 27. 736 & 470.480 & 330.843 & 377.389 & 18. 734 & 20.904 & 21.446 & 5.156 & 7. 163 & 6.495 \\
\hline 70 & 27.384 & 27.563 & 28. 768 & 522.948 & 405.192 & 444.444 & 19. 913 & 21.775 & 22.419 & 5. 348 & 7.656 & 6.887 \\
\hline $80 \ldots$ & 28.114 & 28. 601 & 29. 704 & 577.589 & 482.997 & 514.528 & 20.893 & 22. 564 & 23. 272 & 5.586 & 7. 862 & 7. 103 \\
\hline $90 \ldots$ & 28. 786 & 29.527 & 30.545 & 634.706 & 561.671 & 586.016 & 21.734 & 23. 287 & 24.035 & 5.838 & 7.860 & 7.187 \\
\hline 100 & 29.414 & 30.353 & 31.304 & 694.306 & 639.875 & 658.018 & 22.471 & 23. 954 & 24. 724 & 6.079 & 7. 751 & 7. 193 \\
\hline $120 \ldots$ & 30.559 & 31. 739 & 32. 611 & 819.996 & 791. 908 & 801. 270 & 23.725 & 25.139 & 25. 933 & 6. 466 & 7. 454 & 7. 125 \\
\hline 150 & 32.041 & 33.366 & 34. 189 & $1,019.52$ & $1,010.37$ & $1,013.42$ & 25. 244 & 26. 629 & 27.432 & 6.790 & 7.149 & 7. 029 \\
\hline $200 \ldots$ & 34.023 & 35.395 & 36. 202 & $1,364.06$ & $1,362.90$ & 1, 363. 29 & 27.202 & 28. 580 & 29.386 & 6. 947 & 6. 996 & 6. 980 \\
\hline 298.16 & 36.805 & 38. 182 & 38. 988 & $2,048.10$ & $2,048.08$ & $2,048.09$ & 29.936 & 31.313 & 32.119 & 6.977 & 6.978 & 6.978 \\
\hline 300 & 36.848 & 38.225 & 39. 031 & $2,060.93$ & $2,060.92$ & $2,060.92$ & 29. 978 & 31.355 & 32.161 & 6. 977 & 6. 978 & 6. 978 \\
\hline 400 & 38.857 & 40.234 & 41.040 & -...... & -..... & $2,759.18$ & 31.959 & 33.336 & 34.142 & -...- & ..... & 6. 989 \\
\hline $500 \ldots$ & 40.419 & 41. 796 & 42. 602 & & & $3,459.38$ & 33.500 & 34.877 & 35.683 & -.... & ..... & 7. 019 \\
\hline $600 \ldots$ & 41. 704 & 43.081 & 43.887 & - & ....... & $4,164.03$ & 34. 763 & 36.141 & 36.946 & $\ldots$ & $\ldots$ & 7. 079 \\
\hline $700 \ldots$ & 42.802 & 44. 179 & 44. 985 & (....... & - & $4,876.39$ & 35.835 & 37. 212 & 38.018 & $\ldots$ & -... & 7.173 \\
\hline $1,000 \ldots$ & 45.422 & 46.800 & 47.605 & - & - & $7,084.30$ & 38.338 & 39. 716 & 40.521 & -...- & -...- & 7.562 \\
\hline 1,500 & 48.611 & 49.989 & 50.794 & -........ & -...... & $11,027.3$ & 41. 259 & 42.637 & 43.442 & -...- & -.... & 8.178 \\
\hline $2,000 \ldots$ & 51.027 & 52.405 & 53.210 & ........ & . & 15,229 & 43.411 & 44. 789 & 45. 594 & $\ldots$ & ..... & 8. 598 \\
\hline
\end{tabular}




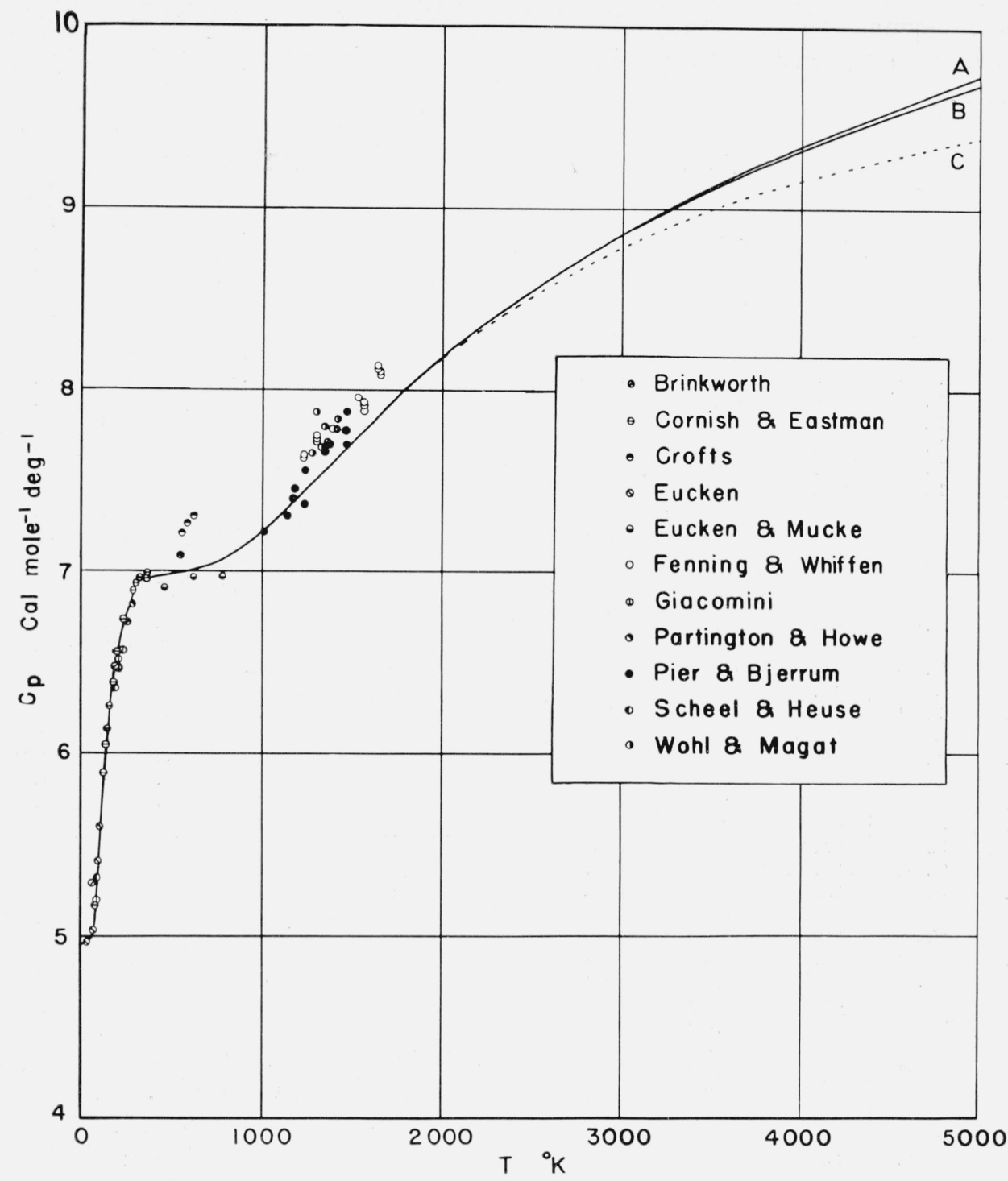

FIGURE 3. Specific heat of normal hydrogen at constant pressure.

T'ABLE 7. Thermodynamic functions for $\mathrm{H}_{2}$ in ideal gaseous state

[Based only on levels below minimum dissociation energy]

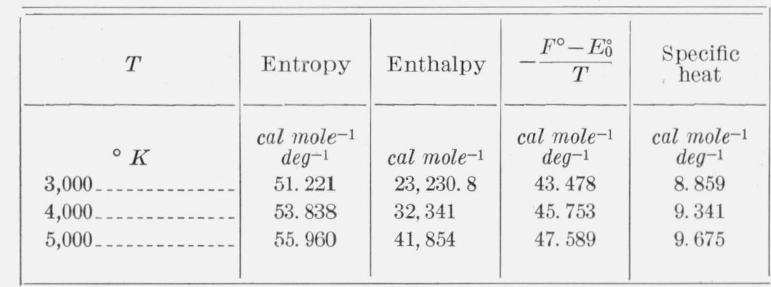

molecular heat capacity if the quantized rotational-vibrational levels above the minimum dissociation energy are included as molecular levels, and curve $\mathrm{B}$ represents the molecular heat capac- ity if the molecular levels are regarded as extending only up to the minimum dissociation energy. In table 7 are tabulated the values of the thermodynamic functions for $n-\mathrm{H}_{2}$ based on calculations involving only energy levels below the minimum dissociation energy.

For convenience in the calculation of the thermodynamic functions of the real gas $n-\mathrm{H}_{2}$, values for $n-\mathrm{H}_{2}$ in the ideal gas state at all temperatures for which there are entries in the tables of PVT data were obtained from table 4 by interpolation and are tabulated in table 8 . The interpolated values of $S^{\circ},-\left(F^{\circ}-E_{0}^{\circ}\right) / \mathrm{T}$, and $C_{p}^{\circ}$ agree to within \pm 0.001 with values that would have been ob- 
tained by direct summation. In the case of $H^{\circ}-E_{0}^{\circ}$, the agreement is within three in the last digit carried.

TABLE 8. Thermodynamic functions for normal $\mathrm{H}_{2}$ in the ideal gaseous state

Values for $\mathrm{S}^{\circ}$ and $-\left(F^{\circ}-E_{0}^{\circ} i / T\right.$ include nuclear spin

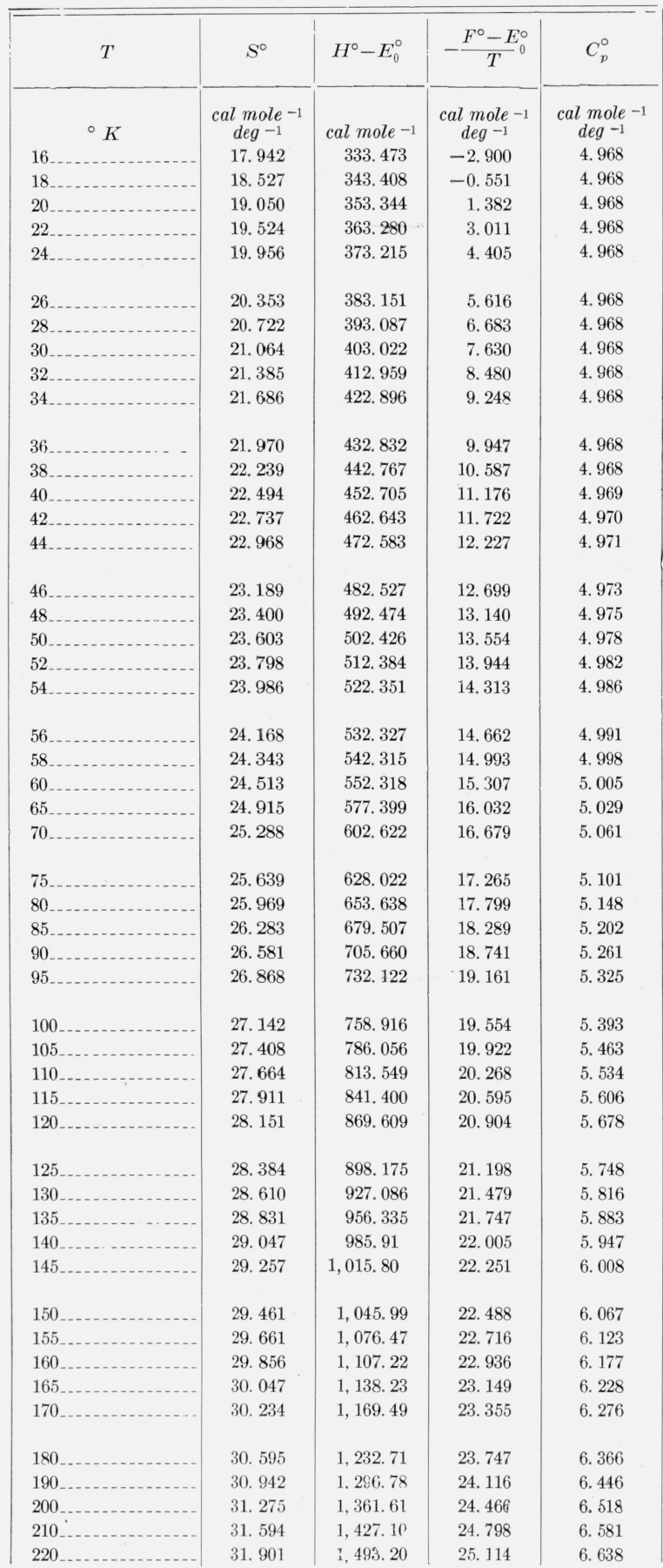

TABLE 8. Thermodynamic functions for normal $\mathrm{H}_{2}$ in the ideal gaseous state-Continued

\begin{tabular}{|c|c|c|c|c|}
\hline$T$ & $S^{\circ}$ & $H^{\circ}-E_{0}^{\circ}$ & $-{\frac{F^{\circ}-E^{\circ}}{T}}_{0}^{\circ}$ & $C_{p}^{\circ}$ \\
\hline${ }^{\circ} K$ & $\mathrm{cal} \mathrm{mole}_{\mathrm{deg}-1}^{-1}$ & cal mole -1 & $\mathrm{cal}_{\mathrm{deg}-1} \mathrm{~mole}^{-1}$ & $\begin{array}{c}\text { eal mole } \\
\mathrm{deg}^{-1}\end{array}$ \\
\hline 230 & 32. 197 & $1,559.85$ & 25.415 & 6. 688 \\
\hline $240 \ldots$ & 32.483 & $1,626.96$ & 25.704 & 6.731 \\
\hline $250 \ldots$ & 32. 758 & $1,694.47$ & 25.981 & 6.770 \\
\hline $260 \ldots \ldots$ & 33.024 & $1,762.33$ & 26.246 & 6.803 \\
\hline 270 & 33. 282 & $1,830.49$ & 26.502 & 6. 831 \\
\hline $280 \ldots \ldots$ & 33.531 & $1,898.92$ & 26. 749 & 6.856 \\
\hline 300 & 34.005 & $2,036.44$ & 27.217 & 6.894 \\
\hline $320 \ldots$ & 34.452 & $2,174.63$ & 27.656 & 6.922 \\
\hline $340 \ldots$ & 34.872 & $2,313.28$ & 28.068 & 6. 943 \\
\hline 360 & 35. 269 & $2,452.29$ & 28.457 & 6.957 \\
\hline $380_{\ldots} \ldots$ & 35.646 & $2,591.53$ & 28.826 & 6.968 \\
\hline 400 & 36.003 & $2,730.95$ & 29.175 & 6.975 \\
\hline $420 \ldots \ldots$ & 36.344 & $2,870.51$ & 29. 509 & 6.980 \\
\hline $440 \ldots$ & 36.668 & $3,010.14$ & 29.826 & 6. 984 \\
\hline 460 & 36.979 & $3,149.85$ & 30.131 & 6.987 \\
\hline $480 \ldots$ & 37.276 & $3,289.62$ & 30.422 & 6.990 \\
\hline $500 \ldots$ & 37.561 & $3,429.46$ & 30. 702 & 6.993 \\
\hline $520 \ldots$ & 37.837 & $3,569.34$ & 30.973 & 6.996 \\
\hline $540 \ldots$ & 38.100 & $3,709.28$ & 31.231 & 6. 999 \\
\hline 560 & 38.355 & $3,849.30$ & 31.481 & 7. 002 \\
\hline $580 \ldots$ & 38.600 & $3,989.36$ & 31. 722 & 7.005 \\
\hline 600 & 38.838 & $4,129.51$ & 31.955 & 7. 009 \\
\hline 650 & 39. 399 & $4,480.19$ & 32.506 & 7. 021 \\
\hline
\end{tabular}

The contributions to the entropy and to the related free energy functions arising from (1) the nuclear spins, (2) the triple degeneracy of the lowest rotational state of $o-\mathrm{H}_{2}$ and $p-\mathrm{D}_{2}$, and (3) the mixing of the ortho and para varieties in $n-\mathrm{H}_{2}$ and $n-\mathrm{D}_{2}$ have been included through eq $2.3,2.4,2.15$, and 2.16 in all the tables. A comparison of the entropies and free energies of hydrogen and deuterium calculated from calorimetric data with values in the tables must take into account the degeneracies existing in the solid state at the lowest temperature of the calorimetric measurements. There must accordingly be added to the calorimetric values of entropy calculated from data extending from $10^{\circ} \mathrm{K}$ to higher temperatures, the entronies of table 9. In calculations concerning chemical reactions above room temperature nuclear spin entropies are customarily omitted for all components of the reactions.

To obtain entropies of $n-\mathrm{H}_{2}, \mathrm{HD}$, and $n-\mathrm{D}_{2}$ suitable for such use above room temperature, there should be subtracted from table values of the entropies $R \ln \left(2 i_{1}+1\right)\left(2 i_{2}+2\right)$ where $i_{1}$ and $i_{2}$ are the two nuclear spins within the molecule [14]. For $n-\mathrm{H}_{2}$ this is equal to $R \ln 4=2.755$ 
TABLE 9. Low-temperature $\left(10^{\circ} \mathrm{K}\right)$ entropy contributions arising from rotational and nuclear-spin degeneracies

\begin{tabular}{|c|c|c|c|c|c|}
\hline \multirow[b]{2}{*}{$\begin{array}{l}\text { Variety } \\
\text { Values of } J \\
\text { Weight of lowest rotational level } \\
(2 J+1) \text {. } \\
\text { Nuclear spin weight, see eq } 2.3 \text { and } \\
2.4 \text {. } \\
\text { Total added entropy }\end{array}$} & \multicolumn{2}{|r|}{$\mathrm{H}_{2}$} & $\begin{array}{l}\text { HD } \\
\text { Only } 1 \\
\text { Both odd and even }\end{array}$ & \multicolumn{2}{|c|}{$\mathrm{D}_{2}$} \\
\hline & $\begin{array}{l}\text { Para } \\
\text { Even } \\
1 \\
1 \\
0\end{array}$ & $\begin{array}{l}\text { Ortho } \\
\text { Odd } \\
3 \\
3 \\
R \ln 9=4.366 \mathrm{cal}^{-1} \\
\mathrm{~mole}^{-1} \mathrm{deg}^{-1} .\end{array}$ & $\begin{array}{l}\text { Only } 1 \\
\text { Both odd and even } \\
1 \\
6 \\
R \ln 6=3.560 \text { cal } \\
\text { mole }{ }^{-1} \mathrm{deg}^{-1} \text {. }\end{array}$ & $\begin{array}{l}\text { Ortho } \\
\text { Even } \\
1 \\
6 \\
R \ln 6=3.560 \text { cal } \\
\text { mole }{ }^{-1} \mathrm{deg}^{-1} \text {. }\end{array}$ & $\begin{array}{l}\text { Para } \\
\text { Odd } \\
3 \\
3 \\
R \ln 9=4.366 \mathrm{cal}^{-1} \\
\mathrm{~mole}^{-1} \mathrm{deg}^{-1} .\end{array}$ \\
\hline & & \multicolumn{2}{|c|}{$n-\mathrm{H}_{2}$} & \multicolumn{2}{|c|}{$n-\mathrm{D}_{2}$} \\
\hline $\begin{array}{l}-R\left(x_{0} \ln x_{o}+x_{p} \ln x_{p}\right) \\
x_{o} s_{o}+x_{p} s_{p} \\
\text { Total added entropy }\left(x_{j} s_{j}-R x_{j} \ln \right.\end{array}$ & $\left.x_{j}\right)$ & \multicolumn{2}{|c|}{$\begin{array}{l}R(\ln 4-3 / 4 \ln 3)=1.117 \mathrm{cal} \mathrm{mole}^{-1} \mathrm{deg}^{-1} \\
3 / 4 R \ln 9=3.275 \mathrm{cal} \mathrm{mole}^{-1} \mathrm{deg}^{-1} \\
R(\ln 4+3 / 4 \ln 3)=4.392 \mathrm{cal} \mathrm{mole}^{-1} \mathrm{deg}-1\end{array}$} & \multicolumn{2}{|c|}{$\begin{array}{l}R(\ln 3-2 / 3 \ln 2)=1.265 \mathrm{cal} \mathrm{mole}^{-1} \mathrm{deg}^{-1} \\
R(4 / 3 \ln 3+2 / 3 \ln 2)=3.829 \mathrm{cal} \mathrm{mole}^{-1} \mathrm{deg}^{-1} \\
7 / 3 R \ln 3=5.094 \mathrm{cal} \mathrm{mole}^{-1} \mathrm{deg}^{-1}\end{array}$} \\
\hline
\end{tabular}

cal mole $\mathrm{deg}^{-1}$; for $\mathrm{HD}, R \ln 6=3.560 \mathrm{cal} \mathrm{mole}^{-1}$ $\mathrm{deg}^{-1}$, and for $n-\mathrm{D}_{2}, R \ln 9=4.366$ cal mole ${ }^{-1}$ $\operatorname{deg}^{-1}$.

The reliability to be expected in thermodynamic functions for the ideal gas state calculated from spectroscopic data has been considered by earlier writers on the basis of the reliability of spectroscopic constants and the gas constant $R$. The former estimate of one or two hundredths of a calorie mole $\mathrm{deg}^{-1}$ for the probable error in the free energy function, specific heat and entropy, appears reasonable. Over much of the temperature range it is probably a more liberal estimate than necessary, as more recent and presumably better spectroscopic data and values for the physical constants have been used. A larger allowance may be necessary for the higher temperatures, however, possibly twice as much at $5,000^{\circ} \mathrm{K}$.

The results of the present calculations below $2,000^{\circ} \mathrm{K}$ are in fairly close agreement with those of Giauque [4], Johnston and Long [18], Davis and Johnston [17], and Wagman, et al. [28]. Above $2,000^{\circ} \mathrm{K}$ the effect of the new calculations of the high rotational levels of $\mathrm{H}_{2}$ is apparent.

This can be seen in figure 3 in which the results of Davis and Johnston (curve $C$ ) for the specific heat of hydrogen, the most sensitive property calculated, are compared with table values of this paper (curves $A$ and $B$ ). Curve $A$, corresponding to table 4, is based on the inclusion of the quantized rotational-vibrational levels above the minimum dissociation energy as molecular levels, and curve $B$, corresponding to table 7 , is based only on levels below the minimum dissociation energy.
In figure 3 are plotted also a large number of scattered points representing the experimental observations of many investigators. [33 to 37 , 40 to $46,50,51,56]$. In cases where mean specific heats were reported, they have been plotted for the mean temperatures of the experimental intervals. At room temperatures and below, the theoretical and experimental specific heats are in good agreement, as has been the case since the correct treatment of the ortho and para forms by Dennison [1] in 1927. Above $1,200^{\circ} \mathrm{K}$ the observations obtained by the explosion method lie above the theoretical curve. The difficulties of the explosion method are great and the accuracy not high [53], consequently the authors feel that the calculated curve and table are more reliable.

At atmospheric pressure and a temperature of $2,000^{\circ} \mathrm{K}$, there is a small but perceptible dissociation of $\mathrm{H}_{2}, \mathrm{HD}$, and $\mathrm{D}_{2}$. As the heat of dissociation of hydrogen is large there are significant differences between the calculated properties of molecular $\mathrm{H}_{2}, \mathrm{HD}$, and $\mathrm{D}_{2}$, tables 4 to 6 , and the properties of the dissociating gases. At $2,000^{\circ}$ $\mathrm{K}$ the table value of $C_{p}$ for molecular $H_{2}$ is 8.195 cal mole ${ }^{-1} \mathrm{deg}^{-1}$, whereas for an ideal gas mixture of molecular and atomic hydrogen in equilibrium at atmospheric pressure the value is 8.797, a difference of $0.60 \mathrm{cal} \mathrm{mole}^{-1} \mathrm{deg}^{-1}$. For HD and $\mathrm{D}_{2}$ the differences between the two specific heats are 0.41 and $0.57 \mathrm{cal} \mathrm{mole}^{-1} \mathrm{deg}^{-1}$, respectively. The effect of pressure upon the specific heat of dissociating hydrogen is illustrated in figure 4 and discussed in section III. At temperatures where there is appreciable dissociation of $\mathrm{HD}$, equilibrium mixtures of $\mathrm{H}_{2}, \mathrm{HD}$, and $\mathrm{D}_{2}$, are established. 


\section{Equilibrium Constants for Dissociation, Isotopic Exchange, and Ortho-Para Conversion}

The equilibrium constant $K$ of a gaseous reaction

$\alpha_{1} A_{1}+\alpha_{2} A_{2}+\alpha_{3} A_{3} \ldots=\beta_{1} B_{1}+\beta_{2} B_{2}+\beta_{3} B_{3} \ldots$,

in which each of the participating gases $A_{1}, A_{2}$, . . ., $B_{1}, B_{2}$, . . . has the equation of state $P V=R T$, is related to the partial pressures of the gases and to their free energies, $F^{*}$, at unit pressure by the equation

$$
\begin{aligned}
R T \ln \frac{\mathrm{P}_{B_{1}}^{\beta_{1}} \mathrm{P}_{B_{2}}^{\beta_{2}} \mathrm{P}_{B_{3}}^{\beta_{3}} \ldots}{\mathrm{P}_{A_{1}}^{\alpha_{1}} \mathrm{P}_{A_{2}}^{\alpha_{2}} \mathrm{P}_{A_{3}}^{\alpha_{3}} \ldots}=R T \ln K= \\
-\left(\Sigma \beta_{j} F_{B_{j}}{ }^{*}-\Sigma \alpha_{j} F_{A_{j}}{ }^{*}\right)=-\Delta F^{*} .
\end{aligned}
$$

Equilibrium constants for dissociation, isotopic exchange,${ }^{6}$ and ortho-para conversion of hydrogen may be calculated by using the $-\left(F^{\circ}-E_{0}^{\circ}\right) / T$ values of tables 4,5 , and $6 . \quad E_{0}^{\circ}$ is the internal energy per mole of molecules without translational motion in the lowest energy level $J=0, v=0$ and in the ideal gas state, and $F^{\circ}$ is for the ideal

${ }^{6}$ Equilibrium $\mathrm{H}_{2}$ and $\mathrm{D}_{2}$. gas state and a pressure of 1 atm: Using $-\left(F^{\circ}-E_{0}^{\circ}\right) / T$ instead of $F^{*}$,

$$
R \ln K=\Delta \frac{-\left(F^{\circ}-E_{0}^{\circ}\right)}{T}-\frac{\Delta E_{0}^{\circ}}{T} .
$$

The values of $\triangle E_{0}{ }^{\circ}$ for the reactions considered in this section are given by the spectroscopic data used in the previous section. Using free energy values as given in the tables of this paper, the atmosphere is the unit of pressure for $K$ and $P$ in the mass action law,

$$
\frac{\mathrm{P}_{B_{1}}^{\beta_{1}} \mathrm{P}_{B_{2}}^{\beta_{2}} \mathrm{P}_{B_{3}}^{\beta_{3}} \ldots}{\mathrm{P}_{A_{1}}^{\alpha_{1}} \mathrm{P}_{A_{2}}^{\alpha_{2}} \mathrm{P}_{A_{3}}^{\alpha_{3}} \ldots}=K .
$$

Deviations from the laws of ideal gases can be taken into account by use of fugacities or activities in place of partial pressures and the forms of eq 3.2, 3.3, and 3.4 for $K$ are retained. When fugacities or activities are substituted for partial pressures, $F^{*}$ becomes the free energy at unit fugacity or activity. For a fuller discussion of the use of fugacities and activities the reader is referred to references [29 to 32 ].

The entropies of monatomic $H$ and $\mathrm{D}$ (see $\mathrm{p}$. 383) must include the nuclear and electron spin entropies besides the entropy of translation, eq

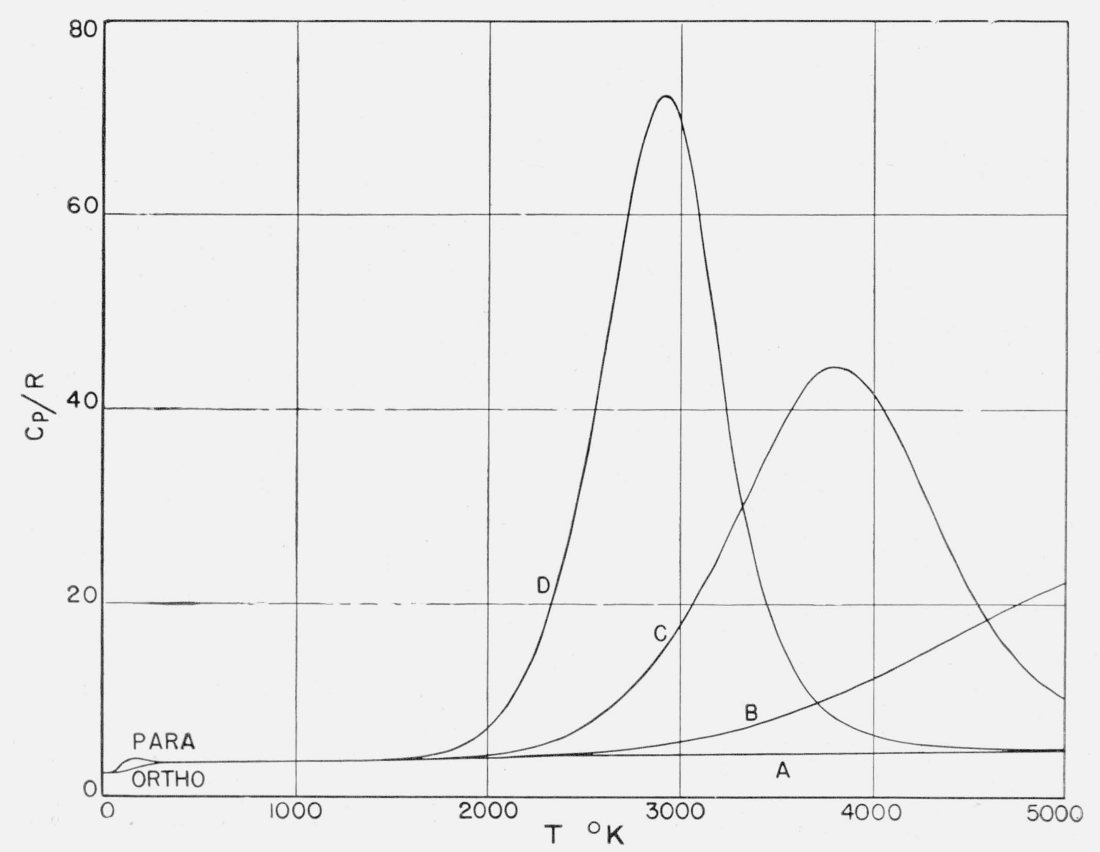

FIgure 4. Curves showing effect of dissociation on specific heat of $\mathrm{H}_{2}$. 
2.12, when used with table values of the entropy and free energy of molecular $\mathrm{H}_{2}, \mathrm{HD}$, and $\mathrm{D}_{2}$, in the calculation of equilibrium constants for dissociation. Accordingly for $\mathrm{H}$,

$$
\begin{aligned}
-\frac{F^{\circ}-E_{0}^{\circ}}{R T}= & \frac{5}{2} \ln T-2.2663 \text { and } \frac{S^{\circ}}{R}= \\
& \frac{5}{2} \ln T+0.2337,
\end{aligned}
$$

and for $D$,

$$
\begin{aligned}
-\frac{F^{\circ}-E_{0}^{\circ}}{R T}= & \frac{5}{2} \ln T-0.8223 \text { and } \frac{S^{\circ}}{R}= \\
& \frac{5}{2} \ln T+1.6777
\end{aligned}
$$

in the ideal gas state at a pressure of 1 atm for the range of temperatures covered by the tables.

\section{Dissociation of $\mathrm{H}_{2}, \mathrm{D}_{2}$, and $\mathrm{HD}$}

The chemical equations for dissociation and the corresponding mass action equations are

$$
\begin{aligned}
& \text { (a) } \mathrm{H}_{2} \leftrightarrows 2 \mathrm{H} ; \quad \frac{P_{\mathrm{H}}^{2}}{P_{\mathrm{H}_{2}}}=K_{\mathrm{H}_{2}} . \\
& \text { (b) } \mathrm{D}_{2} \leftrightarrows 2 \mathrm{D} ; \quad \frac{P_{\mathrm{D}}^{2}}{P_{\mathrm{D}_{2}}}=K_{\mathrm{D}_{2}} . \\
& \text { (c) } \mathrm{HD} \leftrightarrows \mathrm{H}+\mathrm{D} ; \quad \frac{P_{\mathrm{H}} P_{\mathrm{D}}}{P_{\mathrm{HD}}}=K_{\mathrm{HD}} .
\end{aligned}
$$

For these reactions, $\Delta E_{0}^{\circ}$ of eq 3.3 is the difference between the internal energy of 2 moles of dissociated atoms and 1 mole of molecules in the rotational-vibrational state $J=0, v=0$. Beutler's value [21], $36,116 \pm 6 \mathrm{~cm}^{-1}$, was accepted for the dissociation of $\mathrm{H}_{2}$ from its ground state. Assuming that the total depth of the potential energy curve is the same for $\mathrm{H}_{2}, \mathrm{HD}$, and $\mathrm{D}_{2}$, the dissociation energies of $\mathrm{HD}$ and $\mathrm{D}_{2}$ were obtained from the zero-point vibrational energies. These zero point energies were calculated by adding to $G_{0}$ (see eq 2.17), the term which Dunham [10] included in the energy of the ground state relative to the bottom of the potential energy curve and designated $Y_{00}$ in his system. The values thus obtained for the zero point energies of $\mathrm{H}_{2}, \mathrm{HD}$, and $\mathrm{D}_{2}$ were respectively $2,179.6,1,891.0,1,546.6$ $\mathrm{cm}^{-1}$, and the corresponding energies of dissociation for $\mathrm{HD}$ and $\mathrm{D}_{2}$ from the ground state 36,404 . and $36,749 .{ }_{0} \mathrm{~cm}^{-1}$, respectively.
The heats of dissociation of $\mathrm{H}_{2}, \mathrm{HD}$, and $\mathrm{D}_{2}$ in the ideal gas state at temperature $T$ are equal to $\Delta E_{0}^{\circ}+5 R T-\left(H^{\circ}-E_{0}^{\circ}\right)$, where $\left(H^{\circ}-E_{0}^{\circ}\right)$ is the table value of the enthalpy at temperature $T$. The heats of dissociation at $0^{\circ}$ and $298.16^{\circ} \mathrm{K}$ are given in table 11 . The theoretical value for the heat of dissociation of $n-\mathrm{H}_{2}$ at $298^{\circ} \mathrm{K}$ agrees well with the calorimetric value $105,000 \pm 3,500$ cal mole ${ }^{-1}$ obtained by Bichowsky and Copeland [47].

On the assumption that the atomic and molecular forms of hydrogen and deuterium are individually ideal gases, the fraction of the originally totally nondissociated hydrogen which has dissociated is $\sqrt{K /(K+4 P)}$, where $K$ is the dissociation constant and $P$ is the total pressure in atmospheres.

The dissociation constants $K$ and fractions of originally undissociated diatomic molecules, dissociated at 1-atmosphere pressure, are given in table 10 for $\mathrm{H}_{2}, \mathrm{HD}$, and $\mathrm{D}_{2}$.

The experimental values of the equilibrium dissociation constants of $\mathrm{H}_{2}$ as determined by Langmuir and Mackay [32], and by Langmuir [39], are in agreement with the theoretical values of table 10. Langmuir's $x$-values are 0.17 percent at

TABLE 10. Dissociation constants, $K$, and fraction dissociated, $x$, at 1-atm pressure

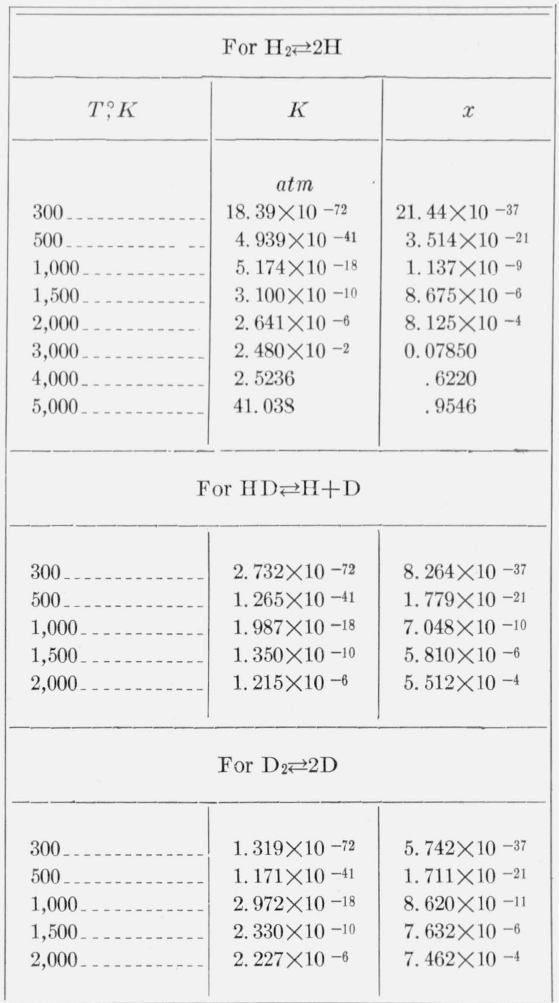


$2,000^{\circ} \mathrm{K}, 1.6$ percent at $2,500^{\circ} \mathrm{K}, 7.2$ percent at $3,000^{\circ} \mathrm{K}$, and 21 percent at $3,500^{\circ} \mathrm{K}$.

TABLE 11. Heats of dissociation of $\mathrm{H}_{2}, \mathrm{HD}$, and $\mathrm{D}_{2}$ in cal mole ${ }^{-1}$

\begin{tabular}{|c|c|c|c|c|c|c|c|}
\hline$T$ & $p-\mathrm{H}_{2}$ & $0-\mathrm{H}_{2}$ & $n-\mathrm{H}_{2}$ & $\mathrm{HD}$ & $0-\mathrm{D}_{2}$ & $p-\mathrm{D}_{2}$ & $n-\mathrm{D}_{2}$ \\
\hline${ }^{\circ} \mathrm{K}$ & 103,239 & 102,900 & 102,985 & 104,064 & 105,048 & 104,877 & 104,991 \\
\hline $298.16 \ldots$ & 104,191 & 104,173 & 104,177 & 104,992 & 105,962 & 105,962 & 105,962 \\
\hline
\end{tabular}

An equation of state for 1 mole of molecular $\mathrm{H}_{2}, \mathrm{HD}$, or $\mathrm{D}_{2}$ capable of forming 2 moles of atoms when completely dissociated, assuming as before that atoms and molecules individually behave as ideal gases, is

$$
\frac{P V}{R T}=1+\sqrt{\frac{K}{K+4 P}}
$$

or

$$
\frac{P V}{R T}=1-\frac{K V}{8 R T}\left(1-\sqrt{1+16 \frac{R T}{K V}}\right)
$$

where $K$ is a function of $T$ determined by eq 3.3 and $V$ is the volume per $2 N_{0}$ atoms uncombined or combined as molecules.

The thermodynamic properties of an equilibrium mixture of atomic and molecular hydrogen in the ideal gas state can in principle be calculated from the properties of atomic hydrogen at low pressures and the equation of state (eq 3.10) or (eq. 3.11). It is simpler, however, to determine the properties of the mixture from the properties of the atomic and molecular varieties and the fraction dissociated.

The equation given by Epstein [30] for the heat capacity of a reacting gas mixture, when applied to the heat capacity of an equilibrium mixture of atomic and molecular hydrogen, is

$$
\begin{gathered}
\frac{\left(C_{p}^{\circ}\right)_{\mathrm{m} \text { ixture }}}{R}=2 x \frac{\left(C_{p}^{\circ}\right)_{\text {atomic }}}{R}+(1-x) \frac{\left(C_{p}^{\circ}\right)_{\text {molecular }}}{R}+ \\
\frac{\left(1-x^{2}\right) x}{2}\left[2 \frac{\left(H^{\circ}\right)_{\text {atomic }}}{R T}-\frac{\left(H^{\circ}\right)_{\text {molecular }}}{R T}\right]^{2},
\end{gathered}
$$

where $x$ is the fraction of the originally totally nondissociated hydrogen that has dissociated, $\left(C_{p}^{\circ}\right)_{\text {atomic }}$ and $\left(C_{p}^{\circ}\right)_{\text {molecular }}$ are heat capacities per mole of atoms and molecules respectively in the ideal gas state, and $\left(C_{p}^{\circ}\right)_{\text {mixture }}$ is for a mixture containing $2 N_{0}$ of atoms combined or uncombined, the components being in the ideal gas state. $\left(C_{p}^{\circ}\right)_{\mathrm{m} \text { ixture }}$ is a function of $P$ as well as $T$ since $x$ is a function of $P$. In figure 4 , curves $D, C$, and $B$ show the variation of $\left(C_{p}^{\circ} / R\right)_{\mathrm{m} \text { ixture }}$ for $\mathrm{H}_{2}$ with temperature for pressures of $0.01,1$, and 100 atmospheres, respectively. Curve $A$ drawn for comparison is the heat capacity of 1 mole of undissociated $\mathrm{H}_{2}$, that is, $\left(C_{p}^{\circ} / R\right)_{\text {molecular. It }}$ appears from these curves that when dissociation has its greatest importance, thermal effects originating in other ways are likely to be dwarfed by comparison. Wildt [19] has calculated the ratio of specific heats of hydrogen at high temperatures using principles similar to those employed here. The results obtained have application to stellar atmospheres.

\section{Ortho-Para Equilibrium}

$$
\begin{gathered}
o-\mathrm{H}_{2} \leftrightarrows p-\mathrm{H}_{2}, \frac{P_{p-\mathrm{H}_{2}}}{P_{o-\mathrm{H}_{2}}}=\left(\frac{p-\mathrm{H}_{2}}{o-\mathrm{H}_{2}}\right)=K . \\
p-\mathrm{D}_{2} \leftrightarrows 0-D_{2}, \frac{P_{o-\mathrm{D}_{2}}}{P_{p-\mathrm{D}_{2}}}=\left(\frac{o-\mathrm{D}_{2}}{p-\mathrm{D}_{2}}\right)=K .
\end{gathered}
$$

The equilibrium constants of the ortho-para conversion of $\mathrm{H}_{2}$ and $\mathrm{D}_{2}$ in the ideal gas state are independent of $P$. Accordingly, pressure does not appreciably change the ortho-para ratio under equilibrium conditions. Although the lowest rotational levels of the ortho and para varieties differ, $\Delta E_{0}^{\circ}$ for the two reactions (eq 3.13 and eq 3.14) is zero, because in the calculations for both the ortho and para varieties the ground state of the molecule, $J=0$ and $v=0$, was arbitrarily selected as the origin of energies.

In table 12 are given values of the percentage para composition in the ideal gas state of equilibrium mixtures of ortho-para varieties calculated from the state-sums, $\Sigma g_{j} e^{-} \epsilon^{j / k T}$, see eq 2.2 and eq 2.14. These values are in close agreement with earlier values obtained by Harkness and Deming [11] and are in agreement with the variations in the relative intensities of the ortho-para spectral lines and with estimates of the ortho-para compositions based on measurements of thermal conduction from heated wires. The success in explaining the heat capacity of gaseous hydrogen at moderate and low temperatures is also corroborating evidence for table 12 [48]. 


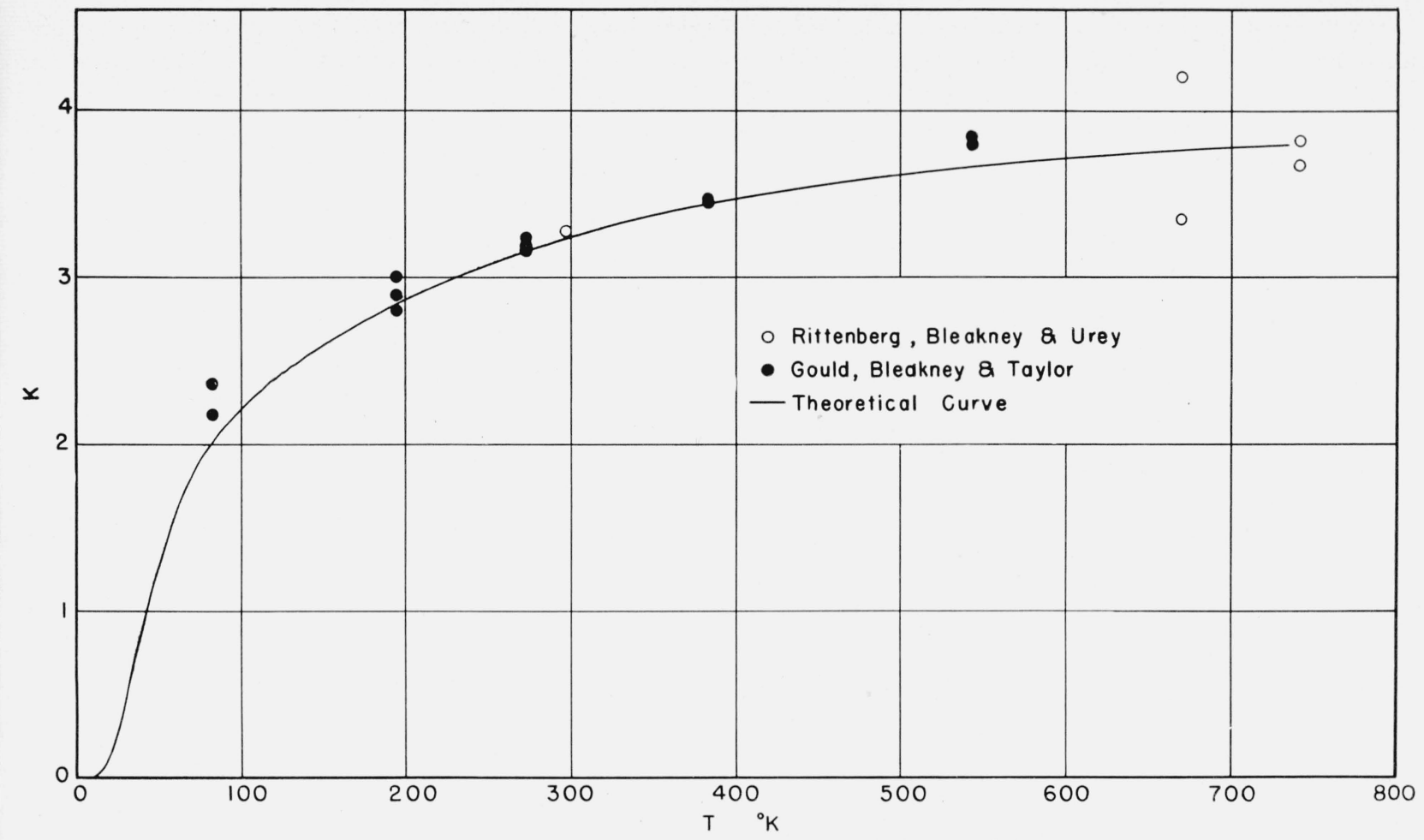

FiguRE 5. The equilibrium constant for $\mathrm{H}_{2}+\mathrm{D}_{2} \rightleftarrows 2 \mathrm{HD}$.

TABLE 12. Ortho-para composition at equilibrium

\begin{tabular}{|c|c|c|}
\hline$T$ & $\begin{array}{l}\text { Percentage } \\
\text { in para form } \\
\text { for } \mathrm{H}_{2}\end{array}$ & $\begin{array}{l}\text { Percentage } \\
\text { in para form } \\
\text { for } D_{2}\end{array}$ \\
\hline${ }^{\circ} K$ & & \\
\hline $10 \ldots$ & 99.9999 & 0.0277 \\
\hline $20 \ldots$ & 99.821 & 1. 998 \\
\hline $20.39 \ldots$ & 99. 789 & - \\
\hline $23.57 \ldots$ & - & 3. 761 \\
\hline $30 \ldots \ldots$ & 97.021 & 7.864 \\
\hline $33.10 \ldots$ & 95.034 & - n \\
\hline $40 \ldots \ldots$ & 88. 727 & 14. 784 \\
\hline $50 \ldots$ & 77. 054 & 20.718 \\
\hline $60 \ldots$ & 65.569 & 25.131 \\
\hline $70 \ldots$ & 55. 991 & 28. 162 \\
\hline $80 \ldots$ & 48.537 & 30.141 \\
\hline $90 \ldots$ & 42. 882 & 31.395 \\
\hline $100 \ldots$ & 38.620 & 32. 164 \\
\hline $120 \ldots$ & 32. 959 & 32. 916 \\
\hline $150 \ldots$ & 28.603 & 33.246 \\
\hline $200 \ldots$ & 25. 974 & 33.327 \\
\hline $250 \ldots$ & 25. 264 & - \\
\hline $298.16 \ldots$ & 25.075 & 33. 333 \\
\hline $300 \ldots$ & 25.072 & 33.333 \\
\hline $350 \ldots$ & 25. 019 & - \\
\hline $400 \ldots$ & 25.005 & - \\
\hline $500 \ldots$ & 25.000 & - \\
\hline
\end{tabular}

\section{Isotopic Exchange}

The chemical and mass action equations for isotopic exchange are

$\mathrm{H}_{2}+\mathrm{D}_{2} \rightleftarrows 2 \mathrm{HD} ; \frac{P_{\mathrm{HD}}^{2}}{P_{\mathrm{H}_{2}} P_{\mathrm{D}_{2}}}=\frac{(\mathrm{HD})^{2}}{\left(\mathrm{H}_{2}\right)\left(\mathrm{D}_{2}\right)}=K_{e x}$
The equilibrium constant $K_{e x}$ of the isotopic exchange reaction (eq 3.15) is related to the dissociation constants $K$ of eq $3.7,3.8$, and 3.9 by the equation

$$
K_{e x}=\frac{K_{\mathrm{H}_{2}} K_{\mathrm{D}_{2}}}{K_{\mathrm{HD}}^{2}}
$$

The equilibrium constant $K_{e x}$ for isotopic exchange in the ideal gas state is independent of $P$, and accordingly the relative equilibrium concentrations of $\mathrm{H}_{2}, \mathrm{HD}$, and $\mathrm{D}_{2}$ are also independent of pressure in the ideal gas state. For this reaction the $\Delta E_{0}^{\circ}$ of eq 3.3 , the difference between twice the energy of the ground state of HD minus the sum of the energies of the ground states of $\mathrm{H}_{2}$ and $\mathrm{D}_{2}$, is equal to twice the zero-point vibrational energy of HD minus the sum of the zeropoint vibrational energies of $\mathrm{H}_{2}$ and $\mathrm{D}_{2}$. Using the values given in section III, 1 for the zero point energies, $\Delta E_{0}^{\circ}$ is 159.5 cal for the formation of 2 moles of HD.

In figure 5 are plotted experimental values of $K_{e x}$, whereas the curve was derived from spectroscopic data as has been indicated. The data of Rittenberg, Bleakney, and Urey [54] were obtained from measurements on hydrogen-deuterium mixtures prepared by the decomposition of mix- 
tures of HI and DI, and those of Gould, Bleakney, and Taylor [55] were obtained with mixtures of hydrogen and deuterium that had been adsorbed on various catalysts or had been diffused through palladium. Some of the observations of Gould, Bleakney, and Taylor plotted in figure 5 were not plotted by them in their published article.

Although the theoretical curve of figure 5 is thought to be more reliable than the experimental data, it is to be pointed out that the uncertainties in the zero-point energies of $\mathrm{H}_{2}, \mathrm{HD}$, and $\mathrm{D}_{2}$ can give rise to perceptible shifts in the curve. Thus a change in $\Delta E_{0}^{\circ}$ of 3 cal mole ${ }^{-1}$, which is equivalent to about $1 \mathrm{~cm}^{-1}$ in $2\left(G_{0}\right)_{H D}{ }^{-}\left(G_{0}\right)_{H 2}{ }^{-}\left(G_{0}\right)_{D 2}$, changes $K_{e x}$ by about 1.5 percent at $100^{\circ} \mathrm{K}$. It seems doubtful that $\Delta E_{0}^{\circ}$ is known better than to a very few calories per mole, for while it is plausible, it is apparently not certain that $D_{e}$, the dissociation energy above the minimum of the potential energy curve, is so nearly the same for $\mathrm{H}_{2}, \mathrm{HD}$, and $\mathrm{D}_{2}$ [25]. The theoretical values of Urey and Rittenberg [13] are, therefore, practically as reliable as the newly calculated ones.

\section{PVT Data and Relations for Hydrogen and Deuterium}

In order to calculate the thermodynamic properties of gaseous hydrogen at high densities (in principle at any densities other than very low) from values of the properties for the hypothetical ideal gaseous state, it is necessary to have information concerning the relations between pressure, volume, and temperature for each temperature in question extending from very low to high densities.

\section{Hydrogen}

The available PVT data for hydrogen fall between $14^{\circ}$ and $700^{\circ} \mathrm{K}$. They consist, in general, of measurements of volume of known amounts of gas at several different pressures along selected isotherms. The quantities usually reported are values of $P V$ or $P V / P_{0} V_{0}$ at the measured pres- sures or densities. In this report this information is presented in the form of tables in which integral values of the variables of state are spaced closely enough to allow accurate interpolation.

The dependent variable $Z$ appearing in the tables is $P V / R T$. Through the definition of $R$, this quantity has the value 1 at extremely low densities, and it is of the same order of magnitude over a very extended range of densities. The independent variables chosen are $T$, the Kelvin temperature, and $\rho$, the Amagat density, which is defined as the ratio of the observed density to the density at standard conditions $\left(0^{\circ} \mathrm{C}\right.$ and 1 atmosphere). Density was chosen as an independent variable of state in preference to pressure because this resulted in simpler representation of the $P V / R T$ isotherms. The Amagat density is also the ratio of the volume $V_{0}$ of the gas at standard conditions to its observed volume.

$$
=\frac{\text { observed density }}{\text { density at standard conditions }}=\frac{V_{0}}{V} .
$$

The best value for $V_{0}$, the molar volume of hydrogen at standard conditions, is 22.4279 liters or $22428.5 \mathrm{~cm}^{3}$, according to the values of $R T_{0}$ obtained by Cragoe [90] and the value of $P V / R T$ for hydrogen at standard conditions as given by Cragoe and the present correlation. The density of hydrogen at standard conditions is 0.089888 gram liter $^{-1}$.

Values of $P V / R T$, or $Z$ for $n-\mathrm{H}_{2}$ are given in table 13 for different values of $T$ and $\rho$. Corresponding values of $P$ and of the derivatives $(d Z / d T)_{\rho},\left(d^{2} Z / d T^{2}\right)_{\rho}$ and $(d Z / d \rho)_{T}$ needed for the calculation of some of the more important thermal properties of the real gas from ideal gas values (see section V) are given as functions of the same variables of state $\rho$ and $T$ in tables $14,15,16$, and 17 , respectively. The temperature intervals used are of graduated size, being as small as 2 degrees at low temperatures and as much as $20 \mathrm{deg}$ above $0^{\circ} \mathrm{C}$. The density intervals, except for entries at $\rho=1,2,3,6$, and 10 , are uniformly equal to 20 Amagats from $\rho=0$ to $\rho=500$. 


\begin{tabular}{|c|c|c|c|c|c|c|c|c|c|c|c|c|c|c|c|}
\hline Temperature & $\rho=1$ & 2 & 3 & 6 & 10 & 20 & 40 & 60 & 80 & 100 & 120 & 140 & 160 & 180 & 200 \\
\hline $16{ }^{\circ} \mathrm{K}$ & 0.990904 & 0.981826 & 0.972765 & & & & & & & & & & & & \\
\hline 18 & $\begin{array}{r}.992299 \\
\end{array}$ & $\begin{array}{l}.984612 \\
\end{array}$ & .976940 & 0.95401 & & & & & & & & & & & \\
\hline $20 \ldots$ & .993373 & .986758 & .980157 & .96043 & 0.93430 & & & & & & & & & & \\
\hline $22 \ldots$ & .994225 & .988460 & .982708 & .96552 & .94274 & & & & & & & & & & \\
\hline $24 \ldots \ldots$ & .994917 & .989846 & .984783 & .96965 & .94962 & 0.90021 & & & & & & & & & \\
\hline $26 \ldots$ & .995492 & .990994 & .986505 & .97309 & .95532 & .91150 & .8265 & & & & & & & & \\
\hline 28 & .995977 & .991962 & .987955 & .97598 & .96012 & .92100 & .8452 & 0.7724 & & & & & & & \\
\hline $30 \ldots \ldots$ & .996389 & .992784 & .989187 & .97844 & .96420 & .92910 & .8610 & .7958 & 0.7334 & 0.6739 & & & & & \\
\hline $32 \ldots$ & .996742 & .993490 & .990245 & .98055 & .96771 & .93606 & .8747 & .8159 & .7598 & .7062 & 0.6551 & 0.6067 & 0.5609 & 0.5176 & \\
\hline $34 \ldots \ldots \ldots$ & .997048 & .994102 & .991162 & .98238 & .97075 & .94209 & .8866 & .8334 & .7826 & .7342 & .6882 & .6446 & .6033 & .5644 & 0.5279 \\
\hline $36 \ldots \ldots$ & .997315 & .994636 & .991962 & .98397 & .97340 & .94735 & .8969 & .8487 & .8025 & .7587 & .7171 & .6777 & .6404 & .6054 & .5726 \\
\hline $38 \ldots \ldots$ & .997550 & .995104 & .992664 & .98537 & .97573 & .95197 & .90598 & .8620 & .8201 & .7803 & .7426 & .7069 & .6732 & .6415 & .6119 \\
\hline $40 \ldots$ & .997758 & .995520 & .993287 & .98662 & .97779 & .95606 & .91403 & .8739 & .8357 & .7995 & .7652 & .7328 & .7023 & .6737 & .6470 \\
\hline \multirow[t]{2}{*}{$42 \ldots$} & .997943 & .995892 & .993844 & .98773 & .97964 & .95972 & .92125 & .8846 & .8497 & .8166 & .7854 & .7560 & .7284 & .7026 & .6786 \\
\hline & $\rho=220$ & 240 & 260 & 280 & 300 & 320 & 340 & 360 & 380 & 400 & 420 & 440 & 460 & 480 & 500 \\
\hline $34 \ldots$ & 0.4937 & 0.4619 & .04327 & 0.4062 & 0.3825 & 0.3613 & 0.3425 & 0.3259 & 0.3115 & 0. 2992 & 0.2890 & 0. 2808 & 0.2748 & 0.2717 & 0.2724 \\
\hline $36 \ldots \ldots$ & .5419 & .5135 & .4875 & .4639 & .4428 & .4239 & .4071 & .3924 & .3800 & .3700 & .3624 & .3573 & .3547 & .3550 & .3590 \\
\hline $38 \ldots \ldots$ & .5844 & .5591 & .5360 & .5151 & .4965 & .4799 & .4652 & .4525 & .4421 & .4343 & .4292 & .4268 & .4272 & .4305 & .4374 \\
\hline $40 \ldots \ldots$ & .6224 & .5998 & .5794 & .5610 & .5446 & .5302 & .5178 & .5073 & .4990 & .4933 & .4905 & .4904 & .4933 & .4992 & .5085 \\
\hline \multirow[t]{2}{*}{42} & .6565 & .6364 & .6183 & .6021 & .5880 & .5758 & .5657 & .5574 & .5512 & .5476 & .5468 & .5488 & .5537 & .5617 & .5730 \\
\hline & $\rho=520$ & 540 & 560 & 580 & 600 & 620 & 640 & 660 & 680 & 700 & & & & & \\
\hline 34 & 0.2775 & 0.2878 & 0.3038 & 0.3258 & 0.3544 & 0.3913 & 0.4385 & 0.4971 & 0.5678 & 0.6512 & & & & & \\
\hline 36 & .3675 & .3809 & .3995 & .4242 & .4557 & .4944 & .5406 & .5947 & & & & & & & \\
\hline 38 & $.44,89$ & .4657 & .4882 & .5167 & .5520 & & & & & & & & & & \\
\hline $40 \ldots \ldots$ & .5225 & .5421 & .5682 & & & & 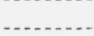 & & & & & & & & \\
\hline $42 \ldots \ldots$ & .5890 & .6110 & & & & & & & & & & & & & \\
\hline
\end{tabular}


TABLE 13. Values of $Z=P V / R T$ for hydrogen at integral values of $T$, the absolute temperature, and $\rho$, the density in Amagat units - Continued

\begin{tabular}{|c|c|c|c|c|c|c|c|c|c|c|c|c|c|c|c|}
\hline Temperature & $\rho=1$ & 2 & 3 & 6 & 10 & 20 & 40 & 60 & 80 & 100 & 120 & 140 & 160 & 180 & 200 \\
\hline${ }_{42}^{\circ} \mathrm{K}$ & 0.997943 & 0.995892 & 0.993844 & 0.98773 & 0.97964 & 0.95972 & 0.92125 & 0.8846 & 0.8497 & 0.8166 & 0.7854 & 0.7560 & 0.7284 & 0.7026 & c. 6786 \\
\hline $44 \ldots$ & $\begin{array}{r}0.999945 \\
.998110\end{array}$ & $\begin{array}{l}.99083226 \\
.99622\end{array}$ & $\begin{array}{l}.939047 \\
.994344\end{array}$ & .98873 & $\begin{array}{l}.98130 \\
\end{array}$ & $\begin{array}{l}. .06302 \\
.96302\end{array}$ & .92775 & .8942 & .8623 & .8321 & .8037 & .7770 & .7520 & .7287 & .7071 \\
\hline $46 \ldots$ & .998261 & .996528 & .994798 & .98963 & .98280 & .96601 & .93364 & .90288 & .8737 & .8462 & .8204 & .7961 & .7734 & .7524 & .7330 \\
\hline 48 & .998399 & .996802 & .995209 & .990453 & .98417 & .96872 & .93898 & .91079 & .8841 & .8590 & .8355 & .8135 & . 7929 & .7740 & .7567 \\
\hline 50 & .998524 & .997052 & .995583 & .991199 & .98541 & .97118 & .94384 & .91798 & .8936 & .8707 & .8493 & .8293 & .8108 & .7938 & .7783 \\
\hline $52 \ldots$ & .998638 & .997280 & . 995925 & .991881 & .98654 & .97342 & . 94827 & .92455 & .90224 & .8814 & .8619 & .8438 & .8272 & .8120 & .7982 \\
\hline 54 & .998743 & .997488 & 996238 & .992507 & .98758 & .97549 & .95235 & .93060 & .91018 & 8911 & .8735 & .8572 & .8422 & .8287 & .8165 \\
\hline 56 & .998839 & 997682 & 996528 & .993086 & .98854 & .97742 & .95615 & .93620 & .91755 & .90020 & .8842 & .8695 & .8560 & .8440 & .8334 \\
\hline 58 & 998930 & 997863 & 996800 & .993628 & 98944 & .97920 & . 95966 & .94138 & .92436 & .90859 & .8941 & .8808 & .8688 & .8582 & .8490 \\
\hline 60 & .999013 & 998030 & .997049 & .994125 & .99027 & .98084 & .96289 & .94615 & .93062 & .91631 & .90323 & .8914 & .8808 & .8715 & .8636 \\
\hline 65. & 999196 & 998394 & .997595 & .995214 & 99208 & .98443 & . 96998 & .95669 & .94454 & .93351 & .92363 & .91492 & .90736 & . 90102 & .8960 \\
\hline 70 & .999348 & .998699 & 998053 & .996129 & 99360 & .98746 & .97597 & .96555 & .95619 & 94793 & .94077 & 93473 & .92983 & .92613 & .9237 \\
\hline 75 & .999479 & .998960 & .998444 & .996910 & .99490 & .99004 & .98106 & .97308 & .96611 & .96020 & .95537 & .95163 & .94904 & .94764 & .9475 \\
\hline 80 & 999590 & .999182 & .998776 & .997573 & .99600 & .99224 & .98543 & .97958 & .97470 & .97082 & .96798 & .96623 & .96561 & .96617 & .9680 \\
\hline 85 & 999686 & 099374 & 999065 & 998151 & 99696 & 99415 & 98923 & 98523 & .98217 & 98007 & .97899 & 97895 & 98002 & .98225 & .9857 \\
\hline 90 . & . 999770 & .999542 & 999318 & .998656 & .99780 & .99583 & .99255 & .99017 & .98871 & 98819 & .98865 & .99011 & .99266 & .99635 & 1. 0012 \\
\hline 95 & $.99984 \dot{4}$ & .999690 & .999539 & $\begin{array}{l}.9980000 \\
.999098 \\
\end{array}$ & .99854 & $\begin{array}{l}.95000 \\
.99730\end{array}$ & $\begin{array}{l}.99548 \\
.9958\end{array}$ & $\begin{array}{r}.99454 \\
.9964\end{array}$ & .99449 & .99536 & .99719 & .99999 & 1. 00386 & 1. 00884 & 1. 0150 \\
\hline 100 & 999909 & .999821 & .999735 & .999489 & 99919 & .99860 & .99807 & .99841 & .99962 & 1. 00173 & 1.00477 & 1.00878 & 1. 01385 & 1.02004 & 1. 0274 \\
\hline 105 & .999968 & 999940 & 999911 & .999841 & .99978 & .99977 & 1.00038 & 1.00186 & 1. 00420 & 1. 00741 & 1.01154 & 1.01665 & 1. 02280 & 1.03006 & 1.0385 \\
\hline $\begin{array}{l}110 \ldots \ldots \\
110 \ldots\end{array}$ & $\begin{array}{r}.000000 \\
1.00021\end{array}$ & $\begin{array}{r}1.000046 \\
1.03\end{array}$ & $\begin{array}{r}\text { 1. } 000070 \\
\text { S }\end{array}$ & $\begin{array}{r}.35000158 \\
1.001\end{array}$ & 1. 00031 & 1. 00082 & $\begin{array}{l}1.00246 \\
1\end{array}$ & $\begin{array}{l}1.00495 \\
\end{array}$ & 1. 00830 & 1. 01250 & 1. 01762 & 1. 02371 & 1. 03084 & 1. 03905 & 1. 0484 \\
\hline 115 & 1. 000068 & 1. 000141 & 1. 000212 & 1. 000442 & 1. 00078 & 1. 00176 & 1. 00434 & 1.00775 & 1. 01200 & 1. 01710 & 1. 02311 & 1. 03009 & 1. 03808 & 1. 04713 & 1.0573 \\
\hline $120 \ldots$ & 1. 000112 & 1. 000228 & 1. 000343 & 1. 000703 & 1. 00121 & 1. $\mathrm{C} 0262$ & 1. 00604 & 1.01028 & 1. 01535 & 1. 02127 & 1. 02809 & 1.03586 & 1.04464 & 1.05445 & 1. 0653 \\
\hline $125 \ldots$ & 1. 000152 & 1. 000307 & 1. 000463 & 1. 000943 & 1. 00161 & 1. 00341 & 1. 00760 & 1. 01260 & 1. 01842 & 1. 02509 & 1. 03265 & 1. 04114 & 1. 05062 & 1. 06113 & 1. 0727 \\
\hline 130 & 1. 000188 & 1. 000379 & 1. 000572 & 1. 001160 & 1. 00197 & 1. 00413 & 1. 00903 & 1. 01473 & 1. 02124 & 1. 02860 & 1. 03684 & 1. 04599 & 1. 05610 & 1. 06725 & 1. 0794 \\
\hline $135 \ldots$ & 1. 000221 & 1. 000444 & 1. 000672 & 1. 001356 & 1. 00230 & 1. 00479 & 1.01035 & 1. 01668 & 1. 02383 & 1. 03183 & 1. 04069 & 1. 05044 & 1. 06113 & 1.07285 & 1. 0856 \\
\hline $140 \ldots$ & 1. 000252 & 1. 000505 & 1. 000763 & 1. 001542 & 1. 00261 & 1. 00540 & 1.01155 & 1. 01847 & 1. 02621 & 1. 03479 & 1. 04422 & 1.05453 & 1.06578 & 1. 07797 & 1. 0912 \\
\hline 145 & 1. 000280 & 1. 000562 & 1. 000847 & 1. 001710 & 1. 00289 & 1. 00596 & 1.01267 & 1. 02014 & 1. 02842 & 1. 03753 & 1.04748 & 1. 05830 & 1. 07002 & 1. 08269 & 1. 0964 \\
\hline 150 & 1. 000307 & 1. 000615 & 1. 000926 & 1. 001868 & 1. 00315 & 1. 00648 & 1.01370 & 1. 02168 & 1. 03047 & 1. 04007 & 1. 05050 & 1. 06179 & 1.07396 & 1. 08707 & 1. 1012 \\
\hline 155 & 1. 000332 & 1. 000665 & 1. 001000 & 1. 002016 & 1. 00340 & 1. 00697 & 1. 01467 & 1. 02312 & 1. 03237 & 1. 04242 & 1. 05330 & 1. 06502 & 1. 07762 & 1. 09114 & 1. 1057 \\
\hline $160 \ldots$ & 1. 000355 & 1. 000711 & 1.001069 & 1. 002154 & 1. 00363 & 1. 00743 & 1.01558 & 1.02447 & 1. 03413 & 1. 04460 & 1. 05590 & 1.06803 & 1. 08102 & 1. 09192 & 1. 1098 \\
\hline 165 & 1. 000376 & 1. 000754 & 1. 001133 & 1. 002282 & 1. 00384 & 1.00785 & 1. 01641 & 1. 02571 & 1. 03577 & 1. 04663 & 1. 05831 & 1. 07082 & 1. 08417 & 1. 09841 & 1. 1136 \\
\hline 170 & 1. 000396 & 1. 000793 & 1. 001192 & 1. 002398 & 1. 00403 & 1. 00823 & 1. 01716 & 1. 02684 & 1. 03728 & 1. 04850 & 1. 06052 & 1.07338 & 1. 08710 & & 1. 1172 \\
\hline 180 & $\begin{array}{l}1.000430 \\
\text { ent }\end{array}$ & $\begin{array}{l}1.000862 \\
\text { lo }\end{array}$ & $\begin{array}{l}1.001296 \\
\end{array}$ & $\begin{array}{l}1.002607 \\
\end{array}$ & $\begin{array}{l}1.00438 \\
\text { 1 }\end{array}$ & $\begin{array}{l}\text { 1. } 00893 \\
\end{array}$ & $\begin{array}{l}1.01856 \\
1\end{array}$ & $\begin{array}{l}1.02891 \\
1.02\end{array}$ & $\begin{array}{l}1.04001 \\
\text { 1 }\end{array}$ & 1. 05186 & 1. 06451 & 1.07797 & 1. 09226 & 1. 1074 & 1. 1235 \\
\hline 190 & $\begin{array}{l}1.000460 \\
\text { 1. }\end{array}$ & $\begin{array}{l}1.000925 \\
\end{array}$ & $\begin{array}{l}1.001390 \\
\end{array}$ & 1. 002795 & $\begin{array}{l}1.00469 \\
1.04\end{array}$ & $\begin{array}{l}\text { 1. } 009555 \\
\text { L }\end{array}$ & $\begin{array}{l}1.01978 \\
1.01978\end{array}$ & $\begin{array}{l}\text { 1. } 0307072 \\
\text { 1. }\end{array}$ & $\begin{array}{l}1.04238 \\
\text { 1 }\end{array}$ & 1. 05478 & $\begin{array}{l}1.06796 \\
\end{array}$ & 1. 08193 & 1. 09671 & 1. 1124 & 1. 1289 \\
\hline $200 \ldots$ & 1. 000490 & 1. 000981 & 1. 001474 & 1. 002962 & 1. 00497 & 1.01010 & 1. 02085 & 1. 03231 & 1.04447 & 1. 05735 & 1.07100 & 1. 08541 & 1. 1006 & 1. 1167 & 1. 1336 \\
\hline & 1. 000515 & 1. 001031 & 1. 001548 & 1. 003108 & 1. 00521 & 1. 01058 & 1. 02181 & 1. 03372 & 1. 04632 & 1. 05964 & 1. 07370 & 1. 08850 & 1. 1041 & 1. 1205 & 1. 1378 \\
\hline $220 \ldots$ & 1. 000537 & 1. 001075 & 1. 001614 & 1. 003240 & $\begin{array}{l}\text { 1. } 00543 \\
\text { S }\end{array}$ & $\begin{array}{l}1.01102 \\
1.02\end{array}$ & $\begin{array}{l}1.02267 \\
1.0267\end{array}$ & $\begin{array}{l}1.03499 \\
1.03\end{array}$ & 1. 04798 & 1. 06167 & 1. 07610 & 1. 09125 & 1. 1072 & 1. 1239 & 1. 1415 \\
\hline $230 \ldots$ & 1. 000556 & 1. 001113 & 1. 001673 & 1. 003358 & 1. 00563 & 1. 01141 & 1. 02344 & 1.03611 & 1. 04945 & 1. 06348 & 1. 07823 & 1. 09370 & 1. 1099 & 1. 1270 & 1. 1448 \\
\hline & 1. 000573 & 1. 001147 & 1. 001725 & 1. 003463 & 1. 00580 & 1. 01175 & 1. 02411 & 1. 03710 & 1.05076 & 1.0 & -1. 08011 & 1. 09587 & 1.1124 & 1. 1297 & 1. 1477 \\
\hline 250 & 1. 000589 & 1. 001178 & 1. 001771 & 1. 003555 & 1. 00596 & 1.01205 & 1. 02470 & 1.03798 & 1. 05191 & 1. 06649 & 1. 08177 & 1. 09777 & 1. 11451 & 1. 1320 & 1. 1502 \\
\hline
\end{tabular}




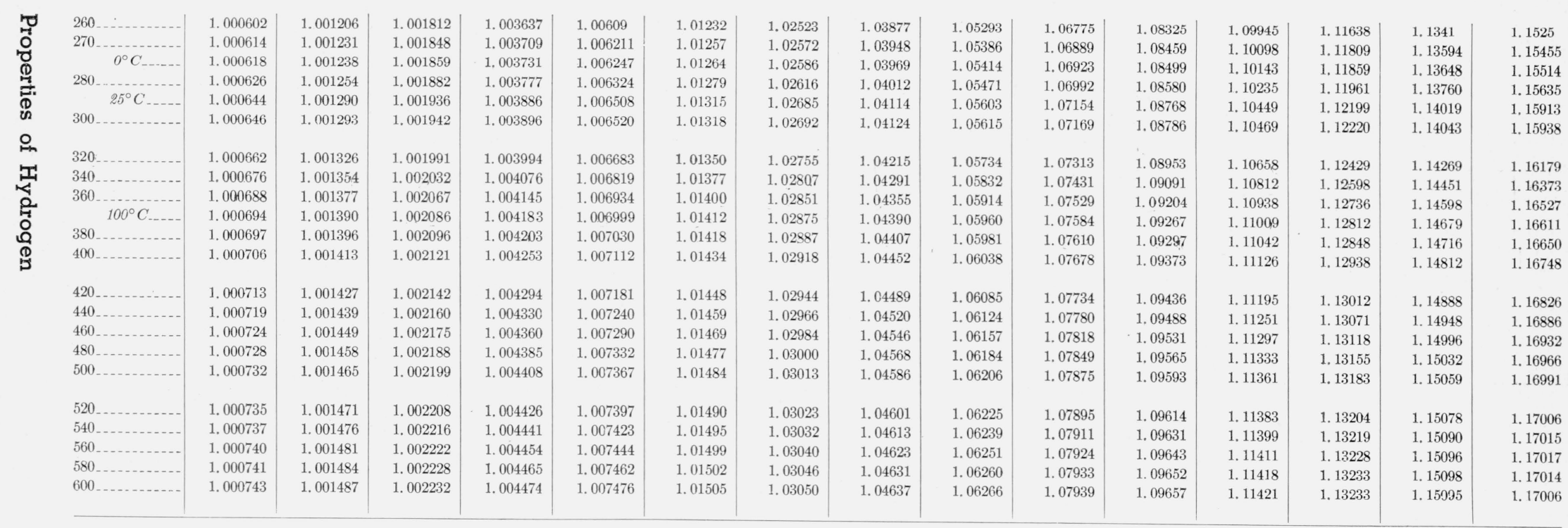


TABLE 13. Values of $Z=P V / R T$ for hydrogen at integral values of $T$, the absolute temperature, and $\rho$, the density in Amagat units-Continued

\begin{tabular}{|c|c|c|c|c|c|c|c|c|c|c|c|c|c|c|c|}
\hline Temperature & $\rho-220$ & 240 & 260 & 280 & 300 & 320 & 340 & 360 & 380 & 400 & 420 & 440 & 460 & 480 & 500 \\
\hline $42 \quad{ }^{\circ} K$ & 0.6565 & 0.6364 & 0.6183 & 0.6021 & 0.5880 & 0.5758 & 0.5657 & 0.5574 & 0.5512 & 0.5476 & 0.5468 & 0.5488 & 0.5537 & 0.5617 & 0.5730 \\
\hline 44 & .6874 & .6695 & .6535 & .6394 & .6274 & .6174 & .6094 & .6033 & .5992 & .5976 & .5987 & .6025 & .6091 & .6187 & .6315 \\
\hline $46 \ldots$ & .7154 & .6996 & .6856 & .6734 & .6633 & .6553 & .6494 & .6454 & .6433 & .6435 & .6464 & .6518 & .6599 & .6707 & .6846 \\
\hline $48 \ldots$ & .7410 & .7270 & .7149 & .7046 & .6962 & .6901 & .6861 & .6840 & .6837 & .6857 & .6902 & .6971 & .7064 & .7183 & .7331 \\
\hline $50 \ldots$ & .7644 & .7522 & .7417 & .7332 & .7265 & .7220 & .7198 & .7194 & .7208 & .7244 & .7304 & .7386 & .7490 & .7620 & .7777 \\
\hline $52 \ldots$ & .7860 & .7754 & .7665 & .7594 & .7544 & .7515 & .7507 & .7518 & .7547 & .7598 & .7672 & .7766 & .7882 & 8022 & .8188 \\
\hline $54 \ldots$ & .8059 & .7968 & .7894 & .7838 & .7802 & .7787 & .7791 & .7815 & .7858 & .7922 & .8008 & .8114 & .8242 & .8392 & .8568 \\
\hline $56 \ldots$ & .8242 & .8166 & .8106 & .8064 & .8041 & .8037 & .8053 & .8088 & .8143 & .8219 & .8316 & .8433 & .8572 & .8733 & .8919 \\
\hline $58 \ldots$ & .8412 & .8350 & .8303 & .8273 & .8262 & .8269 & .8296 & .8342 & .8408 & .8494 & .8601 & .8729 & .8878 & .9049 & .9245 \\
\hline $60 \ldots$ & .8572 & .8522 & .8488 & .8470 & .8470 & .8488 & .8525 & .8582 & .8658 & .8754 & .8871 & .9008 & .9167 & .9348 & .9552 \\
\hline $65 \ldots$ & .8925 & .8906 & .8902 & .8912 & .8938 & .8982 & .9043 & .9123 & .9223 & .9342 & .9482 & .9643 & .9826 & 1.0032 & 1. 0262 \\
\hline $70 \ldots$ & .9228 & .9234 & .9254 & .9289 & .9339 & .9406 & .9489 & .9591 & .9712 & .9852 & 1. 0012 & 1. 0194 & 1. 0397 & 1. 0623 & 1. 0874 \\
\hline $75 \ldots$ & .9488 & .9515 & .9556 & .9611 & .9681 & .9768 & .9872 & .9994 & 1.0133 & 1. 0291 & 1. 0469 & 1. 0668 & 1. 0889 & 1.1132 & 1. 1400 \\
\hline $80 \ldots$ & .9711 & .9756 & .9815 & .9888 & .9976 & 1. 0080 & 1. 0201 & 1. 0339 & 1. 0495 & 1. 0669 & 1. 0861 & 1. 1075 & 1. 1311 & 1. 1568 & 1. 1850 \\
\hline $85 \ldots$ & .9904 & .9965 & 1. 0040 & 1. 0128 & 1. 0232 & 1. 0350 & 1. 0485 & 1. $063 €$ & 1.0806 & 1. 0994 & 1. 1199 & 1. 1425 & 1. 1672 & 1. 1941 & 1. 2234 \\
\hline $90 \ldots$ & 1. 0074 & 1. 0148 & 1.0236 & 1. 0339 & 1.0456 & 1. 0587 & 1. 0733 & 1. 0896 & 1. 1077 & 1. 1275 & 1. 1493 & 1. 1730 & 1.1986 & 1. 2265 & 1. 2568 \\
\hline $95 \ldots$ & 1. 0224 & 1. 0311 & 1. 0411 & 1. 0525 & 1. 0653 & 1. 0795 & 1. 0953 & 1. 1127 & 1. 1317 & 1.1525 & 1. 1752 & 1. 1999 & 1. 2264 & 1. 2551 & 1. 2863 \\
\hline $100 \ldots$ & 1. 0359 & 1. 0457 & 1. 0567 & 1. 0691 & 1. 0829 & 1. 0981 & 1.1148 & 1. 1332 & 1. 1531 & 1. 1748 & 1. 1983 & 1. 2238 & 1. 2511 & 1. 2806 & 1. 3125 \\
\hline 105 & 1. 0481 & 1. 0588 & 1. 0707 & 1. 0840 & 1. 0987 & 1. 1148 & 1. 1323 & 1.1514 & 1. 1722 & 1. 1947 & 1. 2189 & 1. 2450 & 1. 2732 & 1. 3034 & 1. 3358 \\
\hline $110 \ldots$ & 1. 0589 & 1. 0705 & 1. 0833 & 1. 0974 & 1.1128 & 1. 1297 & 1. 1480 & 1. 1678 & 1. 1893 & 1. 2124 & 1. 2373 & 1. 2640 & 1. 2929 & 1. 3236 & 1. 3565 \\
\hline $115 \ldots$ & 1. 0686 & 1. 0810 & 1. 0946 & 1. 1094 & 1. 1256 & 1. 1431 & 1. 1620 & 1. 1825 & 1. 2046 & 1. 2283 & 1. 2538 & 1. 2811 & 1. 3104 & 1.3416 & 1. 3750 \\
\hline $120 \ldots$ & 1.0773 & 1. 0905 & 1. 1048 & 1. 1203 & 1. 1371 & 1. 1552 & 1. 1747 & 1. 1957 & 1. 2184 & 1. 2427 & 1. 2687 & 1. 2965 & 1.3262 & 1. 3579 & 1. 3916 \\
\hline $125 \ldots$ & 1. 0854 & 1. 0991 & 1. 1140 & 1. 1302 & 1. 1475 & 1. 1662 & 1. 1862 & 1. 2077 & 1. 2309 & 1. 2557 & 1. 2821 & 1. 3103 & 1. 3404 & 1. 3725 & 1. 4065 \\
\hline $130 \ldots$ & 1. 0927 & 1. 1070 & 1. 1224 & 1. 1391 & 1. 1570 & 1. 1762 & 1. 1967 & 1. 2187 & 1. 2423 & 1. 2675 & 1. 2943 & 1. 3228 & 1. 3533 & 1. 3856 & 1. 4199 \\
\hline $135 \ldots$ & 1. 0994 & 1. 1142 & 1. 1301 & 1. 1473 & 1. 1656 & 1. 1853 & 1. 2063 & 1. 2287 & 1. 2527 & 1. 2782 & 1. 3053 & 1. 3341 & 1. 3648 & 1. 3974 & 1. 4319 \\
\hline $140 \ldots$ & 1. 1055 & 1. 1208 & 1. 1372 & 1. 1548 & 1. 1736 & 1. 1936 & 1. 2150 & 1. 2378 & 1. 2621 & 1. 2879 & 1.3153 & 1. 3444 & 1. 3753 & 1. 4081 & 1. 4428 \\
\hline $145 \ldots$ & 1. 1111 & 1. 1269 & 1. 1438 & 1. 1618 & 1. 1810 & 1. 2014 & 1. 2231 & 1. 2462 & 1. 2707 & 1. 2968 & 1. 3245 & 1. 3539 & 1. 3850 & 1. 4179 & 1. 4527 \\
\hline $150 \ldots$ & 1. 1163 & 1. 1325 & 1. 1498 & 1. 1681 & 1. 1877 & 1. 2085 & 1. 2305 & 1. 2539 & 1. 2787 & 1. 3051 & 1. 3330 & 1. 3626 & 1. 3939 & 1. 4269 & 1. 4618 \\
\hline 155 & 1. 1212 & 1. 1377 & 1. 1553 & 1. 1740 & 1. 1939 & 1. 2150 & 1. 2373 & 1. 2610 & 1. 2861 & 1. 3127 & 1. 3408 & 1. 3705 & 1. 4019 & 1. 4351 & 1. 4701 \\
\hline $160 \ldots$ & 1. 1257 & 1. 1426 & 1. 1605 & 1. 1795 & 1. 1997 & 1. 2211 & 1. 2436 & 1. 2675 & 1. 2928 & 1. 3195 & 1. 3478 & 1. 3776 & 1. 4091 & 1. 4424 & 1. 4776 \\
\hline $165 \ldots$ & 1. 1299 & 1. 1471 & 1. 1653 & 1. 1846 & 1. 2050 & 1. 2267 & 1. 2495 & 1. 2737 & 1. 2992 & 1. 3260 & 1. 3542 & 1. 3841 & 1.4157 & 1. 4490 & 1. 4841 \\
\hline 170 & 1. 1337 & 1. 1512 & 1. 1697 & 1. 1892 & 1. 2098 & 1. 2316 & 1. 2547 & 1. 2790 & 1.3046 & 1.3316 & 1. 3601 & 1. 3901 & 1. 4216 & 1.4548 & 1. 4899 \\
\hline $180 \ldots$ & 1. 1405 & 1. 1585 & 1. 1774 & 1. 1974 & 1. 2184 & 1. 2406 & 1. 2640 & 1. 2886 & 1. 3145 & 1. 3418 & 1. 3705 & 1. 4007 & 1. 4323 & 1. 4655 & 1. 5005 \\
\hline $190 \ldots$ & 1. 1463 & 1. 1647 & 1. 1840 & 1. 2044 & 1. 2258 & 1. 2483 & 1. 2720 & 1. 2969 & 1. 3230 & 1. 3505 & 1. 3794 & 1. 4097 & 1. 4414 & 1. 4747 & 1. 5097 \\
\hline $200 \ldots$ & 1. 1514 & 1. 1701 & 1. 1898 & 1. 2105 & 1. 2322 & 1. 2550 & 1. 2790 & 1. 3041 & 1. 3305 & 1. 3581 & 1. 3871 & 1. 4175 & 1. 4493 & 1.4827 & 1. 5177 \\
\hline 210 & 1. 1559 & 1. 1749 & 1. 1949 & 1. 2159 & 1. 2379 & 1. 2610 & 1. 2852 & 1. 3105 & 1. 3370 & 1. 3648 & 1. 3939 & 1. 4244 & 1. 4563 & 1.4897 & 1. 5247 \\
\hline $220 \ldots$ & 1. 1599 & 1. 1793 & 1. 1995 & 1. 2207 & 1. 2430 & 1. 2663 & 1. 2906 & 1. 3161 & 1. 3428 & 1.3707 & 1. 3999 & 1. 4304 & 1. 4624 & 1. 4958 & 1. 5307 \\
\hline $230 \ldots$ & 1. 1635 & 1. 1831 & 1. 2036 & 1. 2250 & 1. 2474 & 1. 2709 & 1. 2954 & 1. 3210 & 1. 3478 & 1.3758 & 1. 4051 & 1. 4357 & 1. 4676 & 1. 5010 & 1. 5359 \\
\hline $240 \ldots$ & 1. 1666 & 1. 1864 & 1. 2071 & 1. 2287 & 1. 2512 & 1. 2749 & 1. 2996 & 1. 3253 & 1. 3522 & 1.3802 & 1. 4096 & 1. 4402 & 1.4721 & 1. 5055 & 1. 5403 \\
\hline $250 \ldots$ & 1. 1693 & 1. 1893 & 1. 2102 & 1. 2319 & 1. 2546 & 1. 2784 & 1. 3031 & 1. 3290 & 1. 3559 & 1. 3840 & 1.4134 & 1.4440 & 1. 4759 & 1. 5092 & i. 5440 \\
\hline $260 \ldots$ & 1.1718 & 1.1919 & 1. 2129 & 1. 2348 & 1. 2576 & 1. 2814 & 1. 3062 & 1. 3321 & 1. 3591 & 1. 3872 & 1.4166 & 1.4472 & 1. 4790 & 1. 5122 & 1. 5469 \\
\hline $270 \ldots$ & 1. 17397 & 1. 19420 & 1. 21530 & 1. 23728 & 1. 26020 & 1. 28407 & 1. 30894 & 1. 33486 & 1. 36185 & 1. 38998 & 1. 41927 & 1. 44980 & 1. 48160 & 1. 51473 & 1. 54925 \\
\hline $0^{\circ} C_{-}$ & 1. 17460 & 1. 19487 & 1. 21600 & 1. 23801 & 1. 26094 & 1. 28483 & 1. 30971 & 1. 33563 & 1. 36263 & 1. 39074 & 1. 42003 & 1. 45053 & 1. 48230 & 1. 51539 & 1. 54986 \\
\hline $280 \ldots$ & 1. 17588 & 1. 19623 & 1. 21741 & 1. 23948 & 1. 26245 & 1. 28637 & 1.31127 & 1. 33719 & 1. 36418 & 1. 39227 & 1. 42152 & 1. 45196 & 1. 48366 & 1. 51666 & 1. 55103 \\
\hline
\end{tabular}




\begin{tabular}{|c|c|c|c|c|c|c|c|c|c|c|c|c|c|c|c|}
\hline $25^{\circ} \mathrm{C}$ & 1.17883 & 1. 19932 & 1. 22063 & 1. 24279 & 1. 26583 & 1. 28979 & 1. 31470 & 1. 34060 & 1. 36752 & 1. 39552 & 1. 42463 & 1.45490 & 1. 48638 & 1. 51911 & 1. 55316 \\
\hline $300 \ldots$ & 1. 17910 & 1. 19960 & 1. 22092 & 1. 24308 & 1. 26613 & 1. 29009 & 1.31500 & 1. 34089 & 1. 36781 & 1. 39579 & 1. 42489 & 1. 45514 & 1. 48659 & 1. 51930 & 1. 55331 \\
\hline $320 \ldots$ & 1. 18164 & 1. 20224 & 1. 22364 & 1. 24586 & 1. 26892 & 1. 29287 & 1. 31774 & 1. 34356 & 1.37036 & 1. 39820 & 1. 42710 & 1. 45711 & 1. 48828 & 1. 52065 & 1. 55428 \\
\hline $340 \ldots$ & 1. 18365 & 1. 20432 & 1. 22576 & 1. 24798 & 1. 27104 & 1. 29494 & 1. 31973 & 1. 34544 & 1.37211 & 1. 39976 & 1. 42844 & 1.45820 & 1. 48906 & 1. 52109 & 1. 55431 \\
\hline $360 \ldots \ldots$ & 1. 18525 & 1. 20595 & 1. 22740 & 1. 24961 & 1. 27262 & 1. 29645 & 1. 32114 & 1. 34672 & 1. 37323 & 1. 40069 & 1. 42914 & 1. 45862 & 1. 48917 & 1. 52084 & 1. 55366 \\
\hline $100^{\circ} \mathrm{C}$ & 1. 18612 & 1. 20682 & 1. 22826 & 1. 25045 & 1. 27342 & 1. 29720 & 1. 32182 & 1. 34731 & 1. 37370 & 1. 40102 & 1. 42932 & 1. 45862 & 1. 48897 & 1. 52040 & 1. 55296 \\
\hline $380 \ldots \ldots \ldots$ & 1. 18651 & 1. 20722 & 1. 22865 & 1. 25083 & 1. 27378 & 1. 29753 & 1. 32210 & 1. 34754 & 1. 37387 & 1. 40112 & 1. 42934 & 1. 45854 & 1. 48878 & 1. 52009 & 1. 55252 \\
\hline $400 \ldots$ & 1. 18751 & 1. 20820 & 1. 22960 & 1. 25173 & 1. 27460 & 1. 29825 & 1. 32270 & 1. 34799 & 1. 37414 & 1. 40118 & 1. 42915 & 1. 45809 & 1. 48801 & 1. 51898 & 1. 55101 \\
\hline $420 \ldots$ & 1. 18827 & 1. 20895 & 1. 23031 & 1. 25236 & 1. 27515 & 1. 29869 & 1. 32302 & 1. 34815 & 1.37411 & 1. 40095 & 1. 42868 & 1. 45734 & 1. 48696 & 1. 51758 & 1. 54923 \\
\hline $440 \ldots$ & 1. 18886 & 1. 20950 & 1. 23080 & 1. 25279 & 1. 27549 & 1. 29892 & 1. 32310 & 1. 34807 & 1. 37386 & 1. 40048 & 1. 42797 & 1. 45636 & 1. 48568 & 1. 51598 & 1. 54727 \\
\hline 460 & 1. 18930 & 1. 20989 & 1. 23113 & 1. 25304 & 1. 27564 & 1. 29896 & 1. 32301 & 1. 34782 & 1. 37342 & 1. 39983 & 1. 42709 & 1. 45522 & 1. 48426 & 1. 51422 & 1. 54516 \\
\hline $480 \ldots$ & 1. 18960 & 1. 21015 & 1. 23132 & 1. 25315 & 1. 27565 & 1. 29884 & 1. 32276 & 1. 34741 & 1. 37283 & 1. 39904 & 1. 42607 & 1. 45394 & 1. 48270 & 1. 51236 & 1. 54296 \\
\hline $500_{-}$ & 1. 18980 & 1. 21029 & 1. 23140 & 1. 25314 & 1. 27554 & 1. 29861 & 1. 32238 & 1. 34688 & 1. 37212 & 1.39813 & 1. 42494 & 1. 45257 & 1. 48105 & 1. 51041 & 1. 54068 \\
\hline $520 \ldots$ & 1. 18991 & 1. 21034 & 1. 23138 & 1. 25303 & 1. 27533 & 1. 29828 & 1. 32191 & 1. 34625 & 1. 37132 & 1. 39714 & 1. 42373 & 1.45112 & 1. 47934 & 1. 50841 & 1. 53837 \\
\hline $540 \ldots$ & 1.18995 & 1. 21032 & 1. 23127 & 1. 25284 & 1. 27503 & 1. 29786 & 1. 32136 & 1. 34555 & 1. 37044 & 1. 39607 & 1. 42245 & 1. 44961 & 1. 47758 & 1. 50638 & 1. 53603 \\
\hline $560_{\ldots}$ & 1. 18992 & 1. 21022 & 1. 23110 & 1. 25258 & 1. 27466 & 1. 29738 & 1. 32074 & 1. 34478 & 1. 36951 & 1. 39495 & 1. 42113 & 1. 44807 & 1. 47579 & 1. 50432 & 1. 53369 \\
\hline $580 \ldots$ & 1. 18983 & 1. 21007 & 1. 23088 & 1. 25226 & 1. 27424 & 1. 29684 & 1.32008 & 1. 34397 & 1. 36853 & 1. 39379 & 1. 41977 & 1. 44650 & 1. 47398 & 1. 50226 & 1. 53135 \\
\hline $600 \ldots$ & 1. 18970 & 1. 20987 & 1. 23060 & 1. 25189 & 1. 27377 & 1. 29626 & 1. 31936 & 1. 34311 & 1. 36751 & 1. 39260 & 1. 41839 & 1. 44490 & 1. 47216 & 1. 50019 & 1. 52902 \\
\hline
\end{tabular}


TABLE 14. Pressure (in atmospheres) at integral values of $T$, the absolute temperature, and $\rho$, the density in Amagat units

\begin{tabular}{|c|c|c|c|c|c|c|c|c|c|c|c|c|c|c|c|}
\hline Temperature & $\rho=1$ & 2 & 3 & 6 & 10 & 20 & 40 & 60 & 80 & 100 & 120 & 140 & 160 & 180 & 200 \\
\hline${ }_{16}^{\circ} \mathrm{K}$ & 0.05800 & 0.11495 & 0.17083 & & & & & & & & & & & & \\
\hline 18 & .06535 & .13171 & .19301 & 0.37696 & & & & & & & & & & & \\
\hline $20 \ldots \ldots \ldots$ & .07269 & .14441 & .21516 & .42166 & 0.68364 & & & & & & & & & & \\
\hline 22 & .08002 & .15912 & .23729 & .46628 & .75880 & & & & & & & & & & \\
\hline $24 \ldots \ldots$ & .08736 & .17383 & .25941 & .51085 & .83382 & 1. 58080 & & & & & & & & & \\
\hline $26 \ldots \ldots$ & .09469 & .18853 & .28152 & .55538 & .90873 & 1. 73410 & 3. 1448 & & & & & & & & \\
\hline $28 \ldots$ & .10203 & .20323 & .30362 & .59988 & .98355 & 1. 88695 & 3. 4633 & 4. 7475 & & & & & & & \\
\hline $30 \ldots$ & . 10936 & .21793 & .32571 & .64435 & 1. 05828 & 2. 03952 & 3. 7805 & 5. 2407 & 6. 4397 & 7.3966 & & & & & \\
\hline $32 \ldots \ldots$ & .11669 & .23262 & .35800 & .68879 & 1. 13295 & 2. 19178 & 4. 0967 & 5. 7313 & 7.1163 & 8. 2678 & 9. 2035 & 9. 9441 & 10. 507 & 10. 908 & \\
\hline 34 & .12402 & .24732 & .36988 & .73320 & 1. 20754 & 2. 34377 & 4. 4114 & 6. 2201 & 7. 7879 & 9. 1329 & 10. 273 & 11. 226 & 12.007 & 12. 637 & 13. 133 \\
\hline $36 \ldots \ldots$ & .13136 & .28821 & .39195 & .77759 & 1. 28206 & 2. 4955 & 4. 7252 & 6. 7069 & 8.4557 & 9. 9928 & 11. 334 & 12. 496 & 13.495 & 14. 353 & 15. 083 \\
\hline $38 \ldots$ & .13869 & .27669 & .41402 & .82195 & 1.35652 & 2. 6470 & 5. 0382 & 7. 1904 & 9.1212 & 10.848 & 12.389 & 13.759 & 14. 975 & 16. 053 & 17. 014 \\
\hline $40 \ldots$ & .14602 & .29138 & .43608 & .86632 & 1. 43093 & 2. 7983 & 5.3505 & 7. 6734 & 9. 7839 & 11.700 & 13. 438 & 15.014 & 16. 444 & 17. 746 & 18. 937 \\
\hline \multirow[t]{2}{*}{42} & .15334 & .30606 & .45814 & .91065 & 1. 50532 & 2. 9494 & 5. 6624 & 8.1557 & 10. 445 & 12. 548 & 14. 482 & 16. 263 & 17. 908 & 19. 433 & 20.855 \\
\hline & $\rho=220$ & 240 & 260 & 280 & 300 & 320 & 340 & 360 & 380 & 400 & 420 & 440 & 460 & 480 & 500 \\
\hline $34 \ldots$ & 13. 511 & 13. 790 & 13. 994 & 14. 148 & 14. 274 & 14. 382 & 14. 485 & 14. 594 & 14. 724 & 14.887 & 15. 099 & 15. 369 & 15. 724 & 16. 223 & 16. 942 \\
\hline $36 \ldots$ & 15. 702 & 16. 232 & 16. 694 & 17. 108 & 17:496 & 17. 866 & 18. 230 & 18. 606 & 19. 019 & 19.493 & 20.047 & 20.706 & 21.490 & 22.443 & 23.642 \\
\hline $38 \ldots$ & 17.874 & 18.655 & 19. 375 & 20.051 & 20.708 & 21.350 & 21. 990 & 22.647 & 23. 356 & 24.152 & 25. 061 & 26. 108 & 27.320 & 28.728 & 30.405 \\
\hline $40 \ldots$ & 20.039 & 21.066 & 22. 046 & 22. 988 & 23. 910 & 24.829 & 25.764 & 26. 726 & 27.750 & 28.876 & 30. 148 & 31.577 & 33.208 & 35.066 & 37. 208 \\
\hline \multirow[t]{2}{*}{$42 \ldots$} & 22. 193 & 23.470 & 24.702 & 25.905 & 27. 106 & 28.313 & 29.555 & 30.834 & 32.185 & 33.658 & 35. 289 & 37.105 & 39. 138 & 41.429 & 44. 024 \\
\hline & $\rho=520$ & 540 & 560 & 580 & 600 & 620 & 640 & 660 & 680 & 700 & & & & & \\
\hline $34 \ldots$ & 17. 950 & 19. 332 & 21. 162 & 23.506 & 26.451 & 30.179 & 34. 909 & 40.811 & 48.028 & 56.703 & & & & & \\
\hline $36 \ldots .$. & 25. 170 & 27.091 & 29.466 & 32.405 & 36.012 & 40. 372 & 45. 569 & 51.696 & & & & & & & \\
\hline $38 \ldots$ & 32.453 & 34. 962 & 38.009 & 41.664 & 46.046 & & & & & & & & & & \\
\hline $40 \ldots$ & 39.762 & 42.840 & 46. 565 & & & & & & & & & & & & \\
\hline \multirow[t]{2}{*}{42} & 47.063 & 50. 699 & & & & & & & & & & & & & \\
\hline & $\rho=1$ & 2 & 3 & 6 & 10 & 20 & 40 & 60 & 80 & 100 & 120 & 140 & 160 & 180 & 200 \\
\hline $42 \ldots \ldots$ & 0.15334 & 0.30606 & 0.45814 & 0.91065 & 1. 50532 & 2. 9494 & 5. 6624 & 8. 1557 & 10.445 & 12. 548 & 14. 482 & 16. 263 & 17. 908 & 19. 433 & 20.855 \\
\hline $44 \ldots \ldots$ & .16067 & .32074 & .48020 & .95498 & 1. 57968 & 3.1005 & 5. 9739 & 8. 6368 & 11. 105 & 13.395 & 15. 525 & 17. 511 & 19. 369 & 21.115 & 22.766 \\
\hline $46 \ldots \ldots$ & .16800 & .33542 & .50226 & .99930 & 1. 65400 & 3. 2515 & 6. 2851 & 9.1170 & 11. 763 & 14. 241 & 16. 568 & 18. 757 & 20.826 & 22.792 & 24.672 \\
\hline $48 \ldots \ldots$ & .17533 & .35010 & .52431 & 1. 04361 & 1. 72832 & 3. 4024 & 6. 5959 & 9. 5964 & 12. 421 & 15.085 & 17.607 & 20.000 & 22. 279 & 24.466 & 26. 577 \\
\hline $50 \ldots \ldots$ & .18266 & .36478 & .54636 & 1. 08792 & 1. 80261 & 3. 5531 & 6. 9062 & 10. 076 & 13. 077 & 15. 928 & 18. 643 & 21. 238 & 23. 731 & 26. 138 & 28.475 \\
\hline $52 \ldots$ & .18999 & .37946 & .56841 & 1. 13221 & 1. 87686 & 3. 7038 & 7. 2162 & 10. 554 & 13. 732 & 16. 768 & 19. 677 & 22. 527 & 25.180 & 27. 806 & 30.371 \\
\hline 54 & .19732 & .39414 & .59046 & 1. 17650 & 1. 95110 & 3.8544 & 7. 5260 & 11.031 & 14. 385 & 17.605 & 20.709 & 23. 709 & 26. 622 & 29.470 & 32. 262 \\
\hline $56 \ldots \ldots \ldots$ & .20464 & .40881 & .61251 & 1. 22079 & 2.02533 & 4.0051 & 7.8359 & 11. 509 & 15. 039 & 18. 443 & 21. 739 & 24. 940 & 28.060 & 31.126 & 34.150 \\
\hline $58 \ldots \ldots$ & .21197 & .42349 & .63456 & 1. 26508 & 2. 09957 & 4. 1557 & 8.1455 & 11. 986 & 15. 692 & 19. 280 & 22. 767 & 26. 167 & 29.497 & 32.780 & 36.031 \\
\hline $60 \ldots$ & .21930 & .43817 & .65660 & 1. 30935 & 2. 17380 & 4. 3062 & 8.4548 & 12. 462 & 16. 343 & 20.114 & 23. 793 & 27.395 & 30.936 & 34.435 & 37.915 \\
\hline $65 \ldots$ & .23762 & .47485 & .71171 & 1. 42002 & 2. 35925 & 4. 6821 & 9. 2268 & 13. 651 & 17. 970 & 22.200 & 26.358 & 30.461 & 34. 524 & 38. 569 & 42.615 \\
\hline $70 \ldots$ & .25593 & .51154 & .76681 & 1. 53066 & 2. 54462 & 5. 0578 & 9. 9979 & 14.837 & 19. 590 & 24. 277 & 28.912 & 33.514 & 38.101 & 42.693 & 47. 312 \\
\hline
\end{tabular}




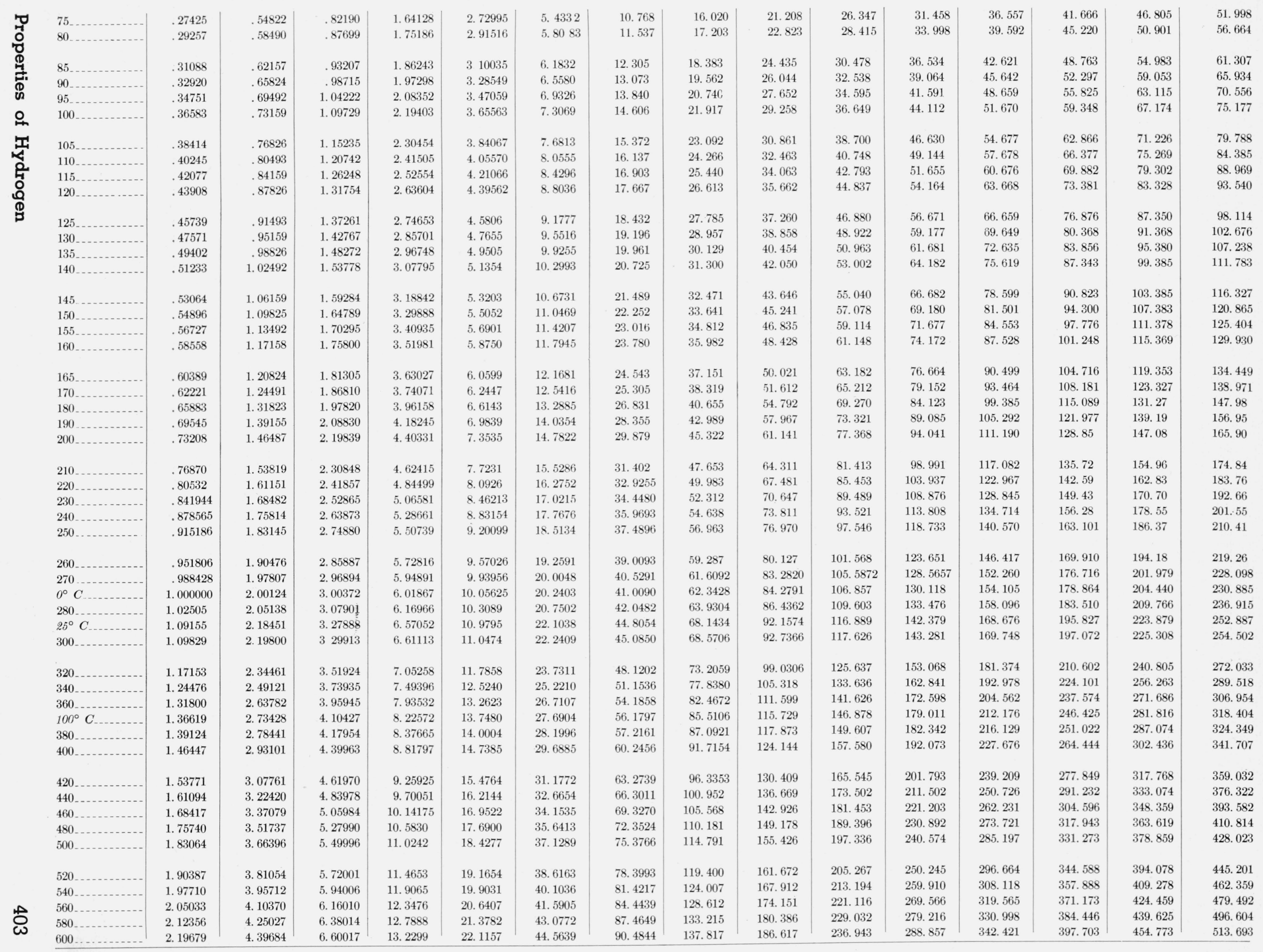


TABLE 14. Pressure (in atmospheres) at integral values of $T$, the absolute temperalure, and $\rho$, the density in Amagat units - Continued

\begin{tabular}{|c|c|c|c|c|c|c|c|c|c|c|c|c|c|c|c|}
\hline Temperature & $\rho=220$ & 240 & 260 & 280 & 300 & 320 & 340 & 360 & 380 & 400 & 420 & 440 & 460 & 480 & 500 \\
\hline $42 \ldots$ & 22. 193 & 23.470 & 24. 702 & 25. 905 & 27. 106 & 28.313 & 29.555 & 30.834 & 32.185 & 33.658 & 35. 289 & 37. 105 & 39.138 & 41.429 & 44. 024 \\
\hline $44 \ldots$ & 24,344 & 25,866 & 27.352 & 28.820 & 30.299 & 31.804 & 33.354 & 34. 962 & 36.654 & 38.480 & 40. 479 & 42.675 & 45.104 & 47.806 & 50.829 \\
\hline $46 \ldots$ & 26,488 & 28. 257 & 30,000 & 31.732 & 33.489 & 35. 291 & 37. 159 & 39. 102 & 41. 140 & 43.319 & 45.690 & 48. 266 & 51.087 & 54. 180 & 57. 607 \\
\hline $48 \ldots$ & 28,628 & 30.641 & 32.642 & 34.646 & 36.678 & 38.781 & 40. 966 & 43. 243 & 45.625 & 48.167 & 50.907 & 53.864 & 57.064 & 60.548 & 64.371 \\
\hline $50 \ldots$ & 30.763 & 33.024 & 35.276 & 37.555 & 39.869 & 42. 264 & 44. 769 & 47.376 & 50.105 & 53. 006 & 56. 117 & 59. 449 & 63. 027 & 66. 908 & 71.132 \\
\hline $52 \ldots$ & 32.897 & 35.404 & 37. 914 & 40.452 & 43. 057 & 45. 751 & 48.558 & 51.490 & 54. 560 & 57.820 & 61. 302 & 65.008 & 68.978 & 73. 256 & 77.887 \\
\hline $54 \ldots$ & 35.028 & 37.780 & 40,549 & 43. 358 & 46. 242 & 49. 230 & 52.333 & 55. 583 & 58. 993 & 62. 604 & 66. 448 & 70.534 & 74. 903 & 79. 582 & 84.636 \\
\hline $56 \ldots$ & 37,150 & 40.154 & 43. 180 & 46. 260 & 49. 423 & 52. 692 & 56.097 & 59.655 & 63.397 & 67.357 & 71.559 & 76.022 & 80.787 & 85.883 & 91.367 \\
\hline 58 & 39. $270 \mathrm{x}$ ? & 42.524 & 45.809 & 49. 154 & 52. 595 & 56.149 & 59.854 & 63. 726 & 67. 798 & 72. 096 & 76.655 & 81.500 & 86. 659 & 92.169 & 98.089 \\
\hline $60 \ldots$ & 41. 397 & 44.897 & 48. 444 & 52.060 & 55. 779 & 59. 624 & 63.626 & 67.820 & 72.222 & 76. 866 & 81.787 & 87.005 & 92. 566 & 98.497 & 104.84 \\
\hline $65 \ldots$ & 46. 694 & 50.830 & 55. 041 & 59. 342 & 63.766 & 68.352 & 73.117 & 78.103 & 83.346 & 88.864 & 94.706 & 100.90 & 107.49 & 114.51 & 122.02 \\
\hline $70 \ldots$ & 51. 993 & 56. 756 & 61.619 & 66.610 & 71.752 & 77. 084 & 82.625 & 88.425 & 94.516 & 100.92 & 107.69 & 114.87 & 122.48 & 130.59 & 139. 24 \\
\hline $75 \ldots$ & 57. 276 & 62.661 & 68.175 & 73.842 & 79. 692 & 85.769 & 92. 100 & 98.723 & 105.66 & 112.95 & 120.65 & 128.80 & 137.44 & 146. 62 & 156.40 \\
\hline $80 \ldots$ & 62. 530 & 68. 531 & 74. 691 & 81.035 & 87.596 & 94.409 & 101.51 & 108. 94 & 115.73 & 124.91 & 133.51 & 142.63 & 152. 29 & 162.52 & 173.42 \\
\hline $85 \ldots$ & 67.759 & 74.374 & 81. 178 & 88.189 & 95.458 & 103.00 & 110.86 & 119.07 & 127.70 & 136.76 & 146. 27 & 156.33 & 166.97 & 178.24 & 190. 23 \\
\hline $9 \theta_{\ldots} \ldots$ & 72.976 & 80.195 & 87. 631 & 95.322 & 103.29 & 111.55 & 120.16 & 129.16 & 138.60 & 148.50 & 158. 94 & 169.94 & 181.55 & 193.85 & 206. 92 \\
\hline $95 \ldots$ & 78.177 & 86. 010 & 94. 081 & 102.43 & 111.08 & 120.06 & 129.43 & 139. 22 & 149.47 & 160.23 & 171.55 & 183.50 & 196.08 & 209.39 & 223.54 \\
\hline $100 \ldots$ & 83.378 & 91.819 & 100.52 & 109. 52 & 118.86 & 128.56 & 138.67 & 149.25 & 160.31 & 171.92 & 184.13 & 197.00 & 210.55 & 224.89 & 240.10 \\
\hline 105 & 88. 579 & 97,618 & 106.94 & 116.60 & 126.62 & 137.04 & 147.89 & 159.23 & 171.12 & 183.58 & 196. 66 & 210.44 & 224.99 & 240.34 & 256. 58 \\
\hline $110_{\ldots}$ & 93.753 & 103.40 & 113.35 & 123,66 & 134.35 & 145.48 & 157.08 & 169.19 & 181.88 & 195.17 & 209.14 & 223.82 & 239.35 & 255.68 & 272.95 \\
\hline $115 \ldots$ & 98.912 & 109.16 & 119.74 & 130.70 & 142.08 & 153.90 & 166.22 & 179.11 & 192.59 & 206.72 & 221.56 & 237.16 & 253.61 & 270.94 & 289.26 \\
\hline $120_{-}$ & 104.05 & 114.90 & 126.11 & 137.72 & 149.77 & 162.29 & 175.35 & 188.98 & 203. 27 & 218.23 & 233.94 & 250.45 & 267.83 & 286.16 & 305.48 \\
\hline $125_{\ldots}$. & 109.20 & 120.64 & 132.46 & 144.72 & 157.43 & 170.67 & 184.44 & 198.83 & 213.91 & 229.70 & 246.26 & 263.66 & 281.98 & 301.28 & 321.61 \\
\hline $130 \ldots$ & 114.34 & 126.36 & 138.80 & 151.70 & 165.09 & 179. 01 & 193. 52 & 208.67 & 224. 53 & 241.14 & 258.55 & 276.82 & 296.08 & 316.33 & 337.66 \\
\hline $135 \ldots$ & 119.46 & 132.08 & 145.12 & 158.67 & 172.71 & 187.34 & 202.57 & 218.47 & 235.11 & 252. 53 & 270.77 & 289.93 & 310.08 & 331.29 & 353.62 \\
\hline $140 \ldots$ & 124.57 & 137.78 & 151.44 & 165.62 & 180.34 & 195.64 & 211. 59 & 228.24 & 245.65 & 263.87 & 282.95 & 302.99 & 324. 04 & 346.19 & 369.50 \\
\hline $145_{\ldots}$ & 129.68 & 143.48 & 157.76 & 172.57 & 187.95 & 203. 95 & 220.61 & 238.00 & 256.16 & 275.18 & 295.11 & 316.02 & 337.98 & 361.05 & 385.32 \\
\hline $150_{\ldots}$. & 134.78 & 149.16 & 164. 06 & 179.49 & 195.54 & 212.23 & 229.60 & 247.72 & 266.66 & 286.49 & 307.24 & 329.02 & 351.88 & 375.87 & 401.11 \\
\hline $155_{\ldots}$ & 139.88 & 154.84 & 170.34 & 186.41 & 203.11 & 220.48 & 238.56 & 257.43 & 277.14 & 297.76 & 319.34 & 341.96 & 265.70 & 390.63 & 416.83 \\
\hline $160 \ldots$ & 144.97 & 160.52 & 176.62 & 193.33 & 210.68 & 228.74 & 247.51 & 267.11 & 287.57 & 308.96 & 331.37 & 354.82 & 379.43 & 405. 29 & 432.47 \\
\hline 165 & 150.06 & 166.19 & 182.90 & 200.23 & 218.23 & 236.97 & 256.46 & 276.80 & 298.03 & 320.19 & 343.34 & 367.64 & 393.12 & 419.86 & 447.80 \\
\hline $170 \ldots$ & 155.13 & 171.84 & 189.15 & 207.10 & 225.73 & 245.12 & 265.33 & 286.38 & 308.34 & 331.28 & 355. 29 & 380.42 & 406. 72 & 434. 32 & 463.33 \\
\hline $180 \ldots$ & 165.24 & 183.10 & 201.60 & 220.79 & 240.71 & 261.44 & 283.02 & 305.50 & 328.95 & 353.45 & 379.07 & 405.87 & 433.89 & 463.25 & 494. 07 \\
\hline $190 \ldots$ & 175.30 & 194.31 & 213. 99 & 234.42 & 255.63 & 277.68 & 300.63 & 324.55 & 349.47 & 375.51 & 402.72 & 431.17 & 460.90 & 492.05 & 524.72 \\
\hline $200 \ldots$ & 185.35 & 205.48 & 226.36 & 248.01 & 270.49 & 293.86 & 318.20 & 343.52 & 369.95 & 397.50 & 426.29 & 456.37 & 487.82 & 520.76 & 555.26 \\
\hline $210 \ldots$ & 195.38 & 216.64 & 238.69 & 261.57 & 285.32 & 310.02 & 335.72 & 362.47 & 390.34 & 419.43 & 449.79 & 481.52 & 514.68 & 549.38 & 585.72 \\
\hline $220 \ldots$ & 205. 39 & 227.81 & 251.02 & 275.11 & 300.14 & 326.15 & 353. 19 & 381.36 & 410.71 & 441.30 & 473. 24 & 506.58 & 541.45 & 577.90 & 616.02 \\
\hline $230 \ldots$ & 215.39 & 238.93 & 263.33 & 288.63 & 314.90 & 342.22 & 370.62 & 400.17 & 430.97 & 463.08 & 496.59 & 531.57 & 568.08 & 606. 27 & 646.21 \\
\hline $240 \ldots$ & 225.36 & 250.02 & 275.58 & 302.08 & 329.59 & 358.22 & 387.98 & 418.93 & 451.18 & 484.76 & 519.84 & 556.42 & 594.59 & 634. 52 & 676.24 \\
\hline $250 \ldots$ & 235.29 & 261.07 & 287.80 & 315.49 & 344.26 & 374.17 & 405.24 & 437.60 & 471.26 & 506.35 & 542.96 & 581.13 & 620.97 & 662.58 & 706.11 \\
\hline $260 \ldots$ & 245.22 & 272.11 & 299. 98 & 328.88 & 358.88 & 390.05 & 422.45 & 456.17 & 491. 27 & 527.82 & 565.96 & 605.72 & 647.16 & 690.46 & 735.73 \\
\hline 270 & 255.128 & 283.117 & 312.130 & 342.219 & 373.456 & 405.898 & 439.629 & 474. 697 & 511.200 & 549.220 & 588.834 & 630.143 & 673.236 & $7: 8.215$ & 765. 191 \\
\hline $0^{\circ} C_{-}$ & 258.252 & 286. 592 & 315.965 & 346.429 & 378.048 & 416.892 & 445.026 & 480.530 & 517.479 & 555.952 & 596.044 & 637.839 & 681.437 & 726.937 & 774.451 \\
\hline $280 \ldots \ldots \ldots$ & 265.008 . & 294. 102 & 324.252 & 355.525 & 387.979 & 421.685 & 456.713 & 493. 138 & 531.041 & 570.500 & 611.610 & 651.455 & 699.141 & 745.765 & 794.443 \\
\hline
\end{tabular}




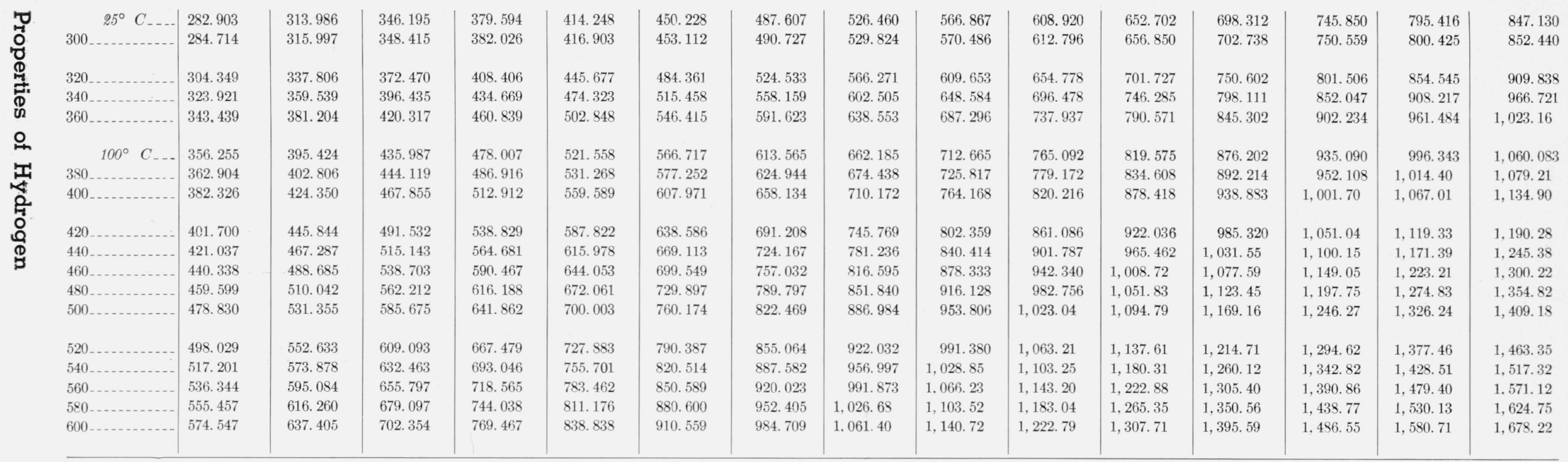


TABLE 15. Values of $(d \boldsymbol{Z} / d T)_{\rho}$ at integral values of $T$, the

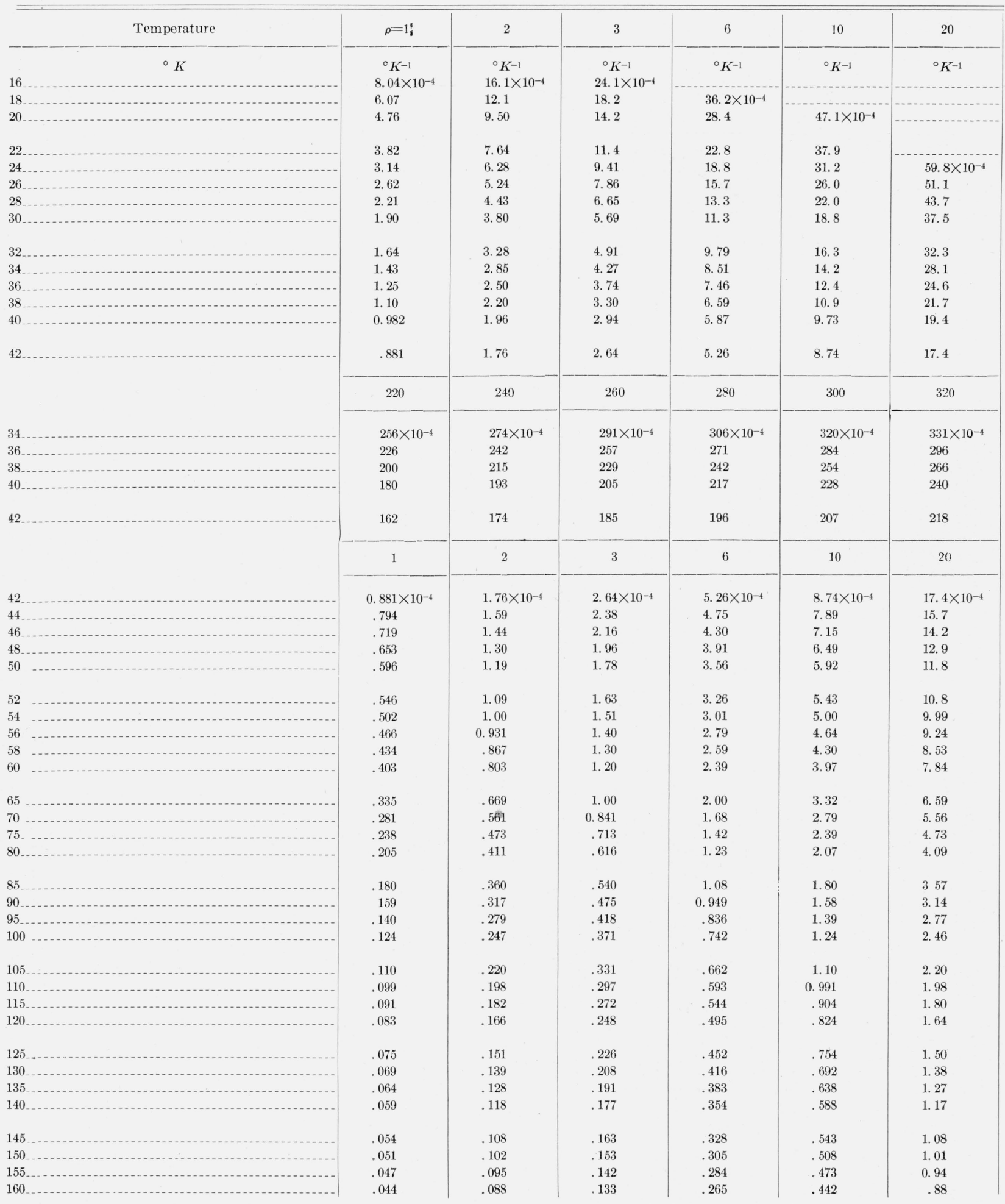


absolute temperature, and $\rho$, the density in Amagat units

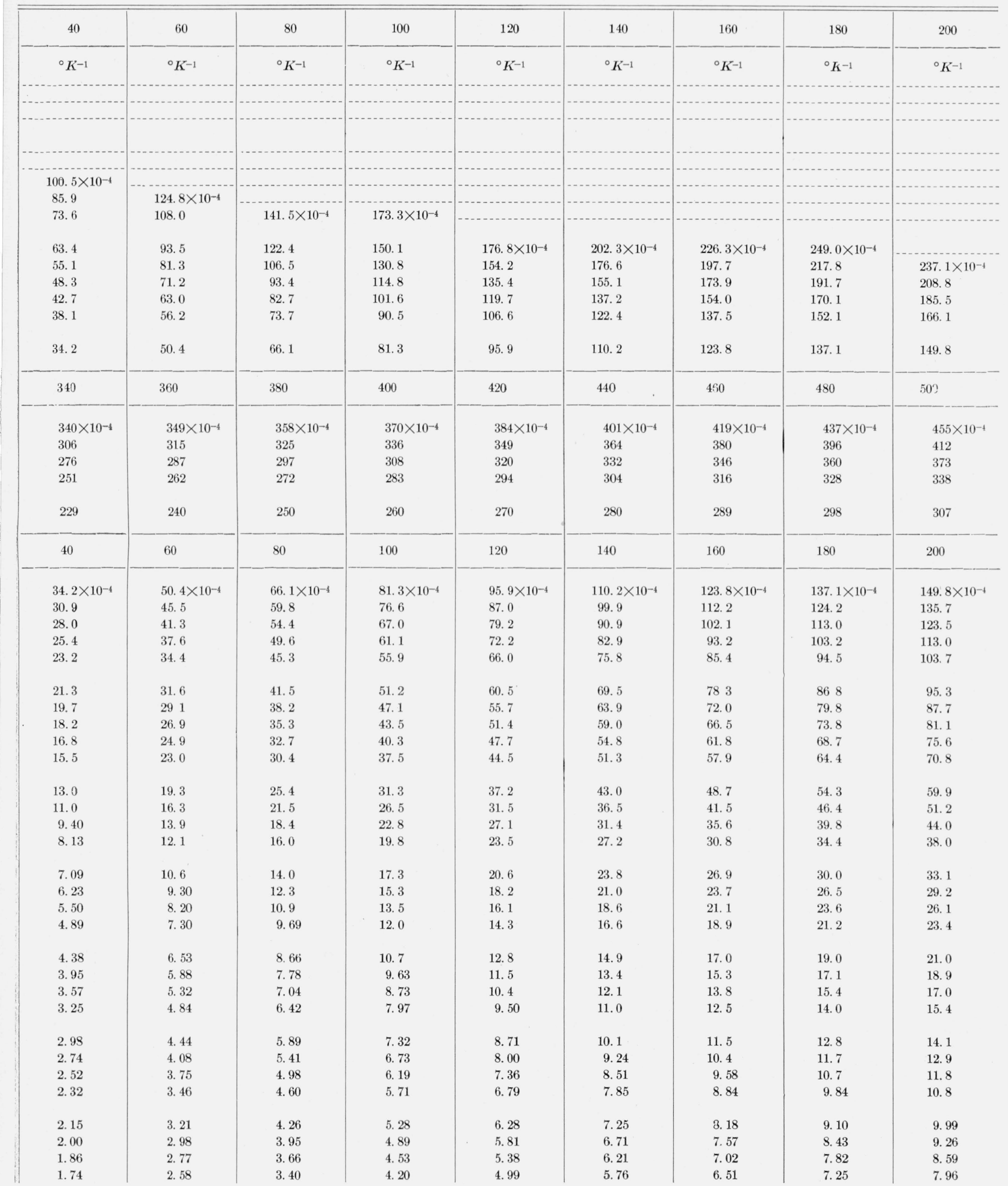


TABLE 15. Values of $(d \boldsymbol{Z} / d T)_{\rho}$ at integral values of $T$, the absolute

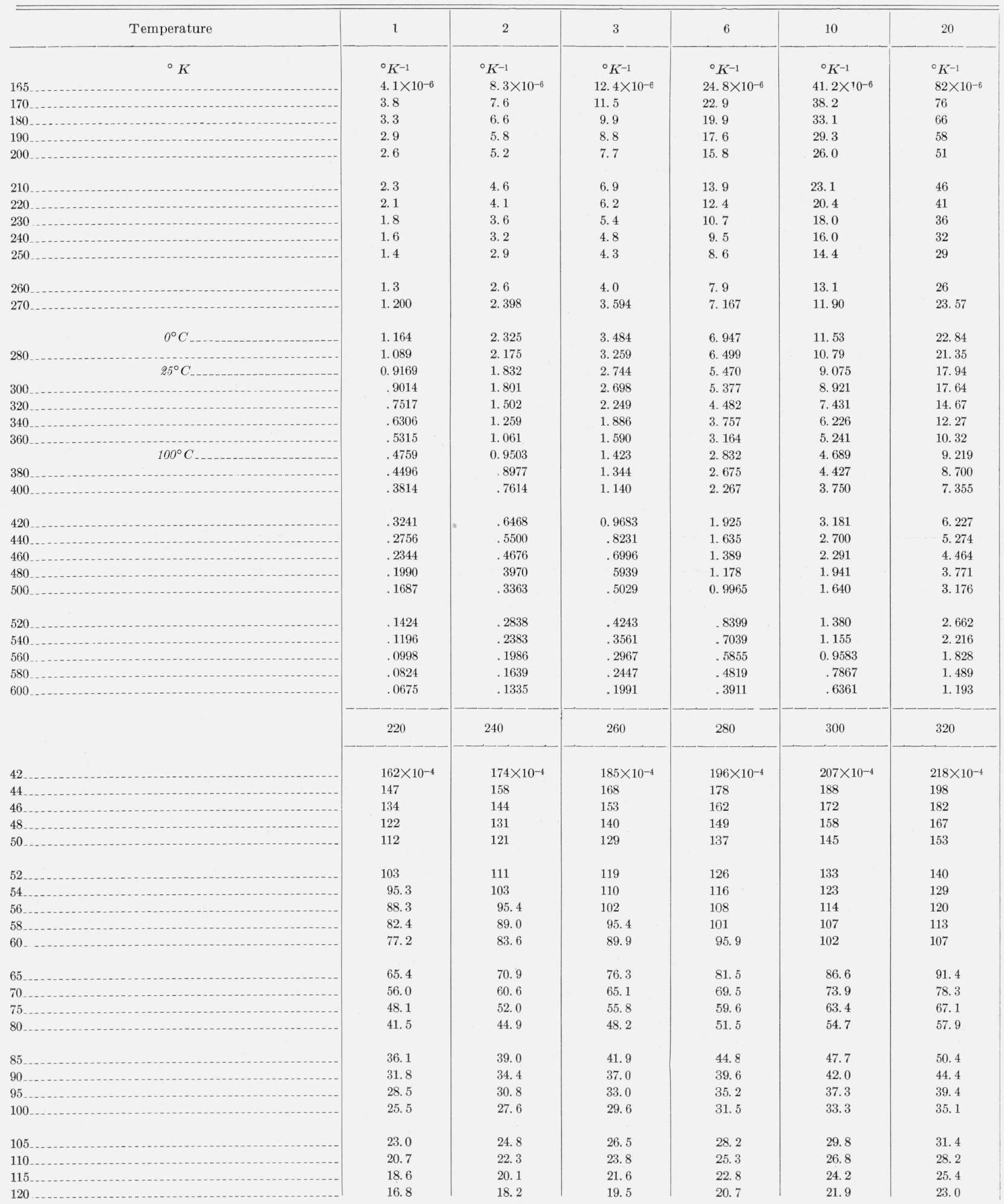


temperature, and $\rho$, the density in Amagat units - Continued

\begin{tabular}{|c|c|c|c|c|c|c|c|c|}
\hline 40 & 60 & 80 & 100 & 120 & 140 & 160 & 180 & 200 \\
\hline${ }^{\circ} K^{-1}$ & ${ }^{\circ} K^{-1}$ & ${ }^{\circ} K^{-1}$ & ${ }^{\circ} K^{-1}$ & ${ }^{\circ} K^{-1}$ & ${ }^{\circ} K^{-1}$ & ${ }^{\circ} K^{-1}$ & ${ }^{\circ} K^{-1}$ & ${ }^{\circ} K^{-1}$ \\
\hline $162 \times 10^{-6}$ & $240 \times 10^{-6}$ & $316 \times 10^{-}$ & $390 \times 10$ & $463 \times 10^{-6}$ & $534 \times 10^{-6}$ & $604 \times 10^{-6}$ & $672 \times 10^{-6}$ & $737 \times 10^{-}$ \\
\hline 151 & 223 & 293 & 361 & 429 & 495 & 559 & 622 & 682 \\
\hline 131 & 194 & 254 & 312 & 370 & 427 & 483 & 536 & 587 \\
\hline 114 & 169 & 222 & 273 & 323 & 372 & 419 & 464 & 507 \\
\hline 101 & 150 & 197 & 242 & 285 & 327 & 367 & 405 & 442 \\
\hline 91 & 134 & 175 & 215 & 253 & 290 & 325 & 358 & 391 \\
\hline 81 & 119 & 156 & 192 & 226 & 258 & 289 & 319 & 348 \\
\hline 71 & 105 & 138 & 170 & 200 & 229 & 257 & 283 & 308 \\
\hline 63 & 93 & 122 & 151 & 178 & 203 & 228 & 251 & 274 \\
\hline 56 & 83 & 109 & 134 & 158 & 180 & 202 & 222 & 242 \\
\hline 51 & 75 & 98 & 120 & 141 & 161 & 180 & 198 & 215 \\
\hline 46. 21 & 67.90 & 88.63 & 108.4 & 127.0 & 144.7 & 161. 2 & 176.6 & 190.7 \\
\hline 44. 76 & 65. 74 & 85.76 & 104.8 & 122.8 & 139. 7 & 155.6 & 170.3 & 183. 7 \\
\hline 41.81 & 61.34 & 79.92 & 97.53 & 114.1 & 129.7 & 144.1 & 157.5 & 169.6 \\
\hline 35.04 & 51.26 & 66.59 & 80.99 & 94.42 & 106.9 & 118.2 & 128.5 & 137.7 \\
\hline 34.43 & 50.36 & 65.40 & 79.51 & 92.66 & 104.8 & 115.9 & 125.9 & 134.8 \\
\hline 28.56 & 41. 63 & 53.87 & 65.24 & 75.70 & 85. 22 & 93. 74 & 101.2 & 107.6 \\
\hline 23.82 & 34.61 & 44.61 & 53.80 & 62.13 & 69.58 & 76.09 & 81.62 & 86. 13 \\
\hline 19.95 & 28.88 & 37.08 & 44.52 & 51.15 & 56.95 & 61.87 & 65.87 & 68.90 \\
\hline 17. 79 & 25. 69 & 32.88 & 39.34 & 45.04 & 49. 94 & 53.99 & 57.16 & 59. 40 \\
\hline 16. 77 & 24.18 & 30.90 & 36.91 & 42.16 & 46.64 & 50.29 & 53.08 & 54.96 \\
\hline 14. 12 & 20.27 & 25.78 & 30.62 & 34.75 & 38.15 & 40.78 & 42.59 & 43.56 \\
\hline 11. 90 & 17. 00 & 21.50 & 25.37 & 28.58 & 31.10 & 32.90 & 33.93 & 34.17 \\
\hline 10. 03 & 14. 25 & 17. 90 & 20.97 & 23.41 & 25.21 & 26.33 & 26.73 & 26. 38 \\
\hline 8. 443 & 11. 92 & 14.86 & 17. 25 & 19.06 & 20.25 & 20.81 & 20.70 & 19.88 \\
\hline 7. 086 & 9. 926 & 12.27 & 14.09 & 15. 36 & 16.06 & 16.15 & 15. 62 & 14.42 \\
\hline 5. 922 & 8. 221 & 10.05 & 11. 39 & 12. 21 & 12.49 & 12.20 & 11.32 & 9. 81 \\
\hline 4. 919 & 6. 753 & 8. 144 & 9.070 & 9. 510 & 9. 439 & 8.833 & 7. 665 & 5. 906 \\
\hline 4. 050 & 5. 484 & 6. 499 & 7. 076 & 7. 192 & 6.825 & 5. 953 & 4. 549 & 2. 588 \\
\hline 3. 295 & 4. 383 & 5. 074 & 5. 350 & 5. 190 & 4. 574 & 3.478 & 1. 880 & -0.246 \\
\hline 2. 636 & 3. 423 & 3. 834 & 3. 852 & 3.456 & 2. 627 & 1. 345 & -0.414 & -2.673 \\
\hline 2. 059 & 2. 585 & 2. 754 & 2. 549 & 1. 951 & 0.943 & -0.496 & -2.387 & -4.751 \\
\hline 340 & 360 & 380 & 400 & 420 & 440 & 460 & 480 & 500 \\
\hline $229 \times 10^{-4}$ & $240 \times 10^{-4}$ & $250 \times 10^{-4}$ & $260 \times 10^{-4}$ & $270 \times 10^{-4}$ & $280 \times 10^{-4}$ & $289 \times 10^{-4}$ & $298 \times 10^{-4}$ & $307 \times 10^{-4}$ \\
\hline 209 & 220 & 230 & 240 & 249 & 257 & 265 & 272 & 279 \\
\hline 192 & 201 & 211 & 220 & 229 & 236 & 243 & 249 & 254 \\
\hline 175 & 184 & 193 & 202 & 210 & 216 & 223 & 228 & 233 \\
\hline 161 & 169 & 177 & 185 & 192 & 198 & 204 & 209 & 214 \\
\hline 148 & 155 & 162 & 169 & 176 & 182 & 188 & 193 & 198 \\
\hline 136 & 143 & 149 & 156 & 162 & 167 & 173 & 179 & 183 \\
\hline 126 & 132 & 138 & 144 & 149 & 155 & 160 & 166 & 171 \\
\hline 118 & 124 & 129 & 134 & 140 & 145 & 150 & 156 & 161 \\
\hline 112 & 117 & 122 & 126 & 131 & 137 & 142 & 148 & 153 \\
\hline 96.1 & 101 & 105 & 109 & 114 & 118 & 123 & 127 & 132 \\
\hline 82.7 & 86.6 & 90.6 & 94.3 & 98.1 & 102 & 106 & 110 & 113 \\
\hline 70.8 & 74.5 & 78.0 & 81.4 & 84.7 & 87.9 & 91.0 & 94.1 & 97.2 \\
\hline 61.0 & 64.1 & 67.2 & 70.3 & 73.2 & 75.8 & 78.5 & 81.0 & 83.5 \\
\hline 53.0 & 55.6 & 58.2 & 60.8 & 63.2 & 65.4 & 67.6 & 69.8 & 72.1 \\
\hline 46.6 & 48.8 & 51.0 & 53.1 & 55.2 & 57.2 & 59.1 & 61.0 & 62.9 \\
\hline 41. 4 & 43.3 & 45. 2 & 47.1 & 48.9 & 50.6 & 52.3 & 53.9 & 55.5 \\
\hline 36.9 & 38.7 & 40.4 & 42.0 & 43.6 & 45.1 & 46.6 & 48.0 & 49.3 \\
\hline 33.0 & 34.5 & 36.0 & 37.5 & 38.9 & 40.3 & 41.6 & 42.8 & 43.9 \\
\hline 29.6 & 30.9 & 32. 2 & 33.5 & 34.8 & 36.0 & 37. 2 & 38.2 & 39.2 \\
\hline 26.6 & 27.8 & 29.0 & 30.2 & 31.3 & 32.3 & 33.3 & 34.2 & 35.0 \\
\hline 24.1 & 25.1 & 26. 2 & 27.3 & 28.3 & 29.1 & 29.9 & 30.6 & 31.3 \\
\hline
\end{tabular}


TABLE 15. Values of $(d Z / d T)_{\rho}$ at integral values of $T$, the absolute

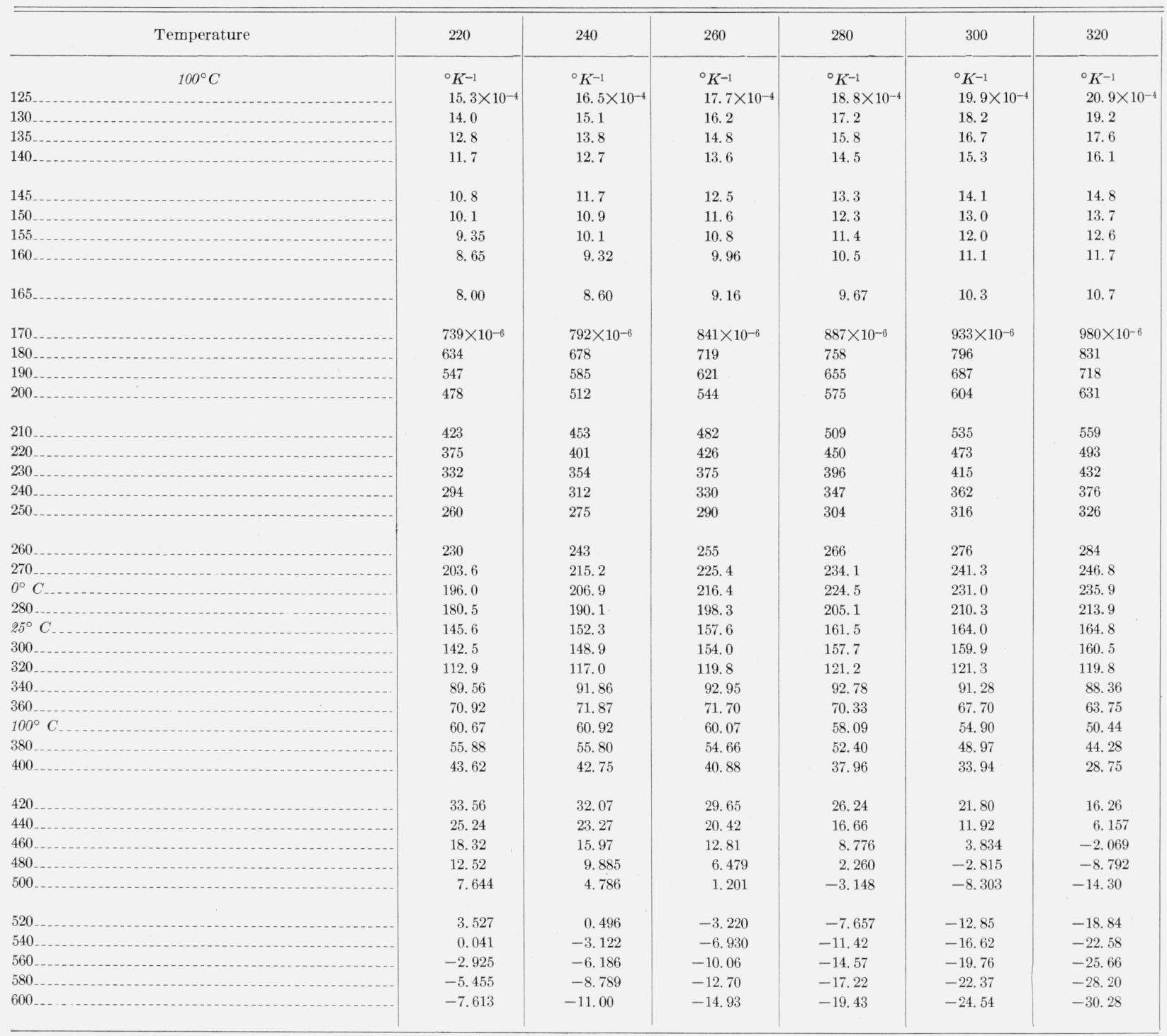


temperature, and $\rho$, the density in Amagat units-Continued

\begin{tabular}{|c|c|c|c|c|c|c|c|c|}
\hline 340 & 360 & 380 & 400 & 420 & 440 & 460 & 480 & 500 \\
\hline${ }^{\circ} K^{-1}$ & ${ }^{\circ} K^{-1}$ & ${ }^{\circ} K^{-1}$ & ${ }^{\circ} K^{-1}$ & ${ }^{\circ} K^{-1}$ & ${ }^{\circ} K^{-1}$ & ${ }^{\circ} K^{-1}$ & ${ }^{\circ} K^{-1}$ & ${ }^{\circ} K^{-1}$ \\
\hline $21.9 \times 10^{-4}$ & $22.9 \times 10^{-4}$ & $23.9 \times 10^{-4}$ & $24.8 \times 10^{-4}$ & $25.6 \times 10^{-4}$ & 26. $3 \times 10^{-4}$ & $27.0 \times 10^{-4}$ & 27. $6 \times 10^{-4}$ & $28.1 \times 10^{-4}$ \\
\hline 20.1 & 21.0 & 21.8 & 22.5 & 23.2 & 23.8 & 24.4 & 24. 9 & 25.3 \\
\hline 18.4 & 19.2 & 19.9 & 20.5 & 21.1 & 21.7 & 22.1 & 22.5 & 22.9 \\
\hline 16. 9 & 17.6 & 18.2 & 18.8 & 19.3 & 19.8 & 20.2 & 20.5 & 20.8 \\
\hline 15.5 & 16.1 & 16.7 & 17. 2 & 17. 7 & 18.1 & 18.5 & 18. 8 & 19.0 \\
\hline 14.3 & .14 .8 & 15.3 & 15.8 & 16.2 & 16.6 & 16.9 & 17.1 & 17. 3 \\
\hline 13. 2 & 13.7 & 14.1 & 14.5 & 14.8 & 15.1 & 15.3 & 15.5 & 15. 6 \\
\hline 12.2 & 12.7 & 13.0 & 13.3 & 13.5 & 13. 7 & 13.9 & 14.0 & 14.0 \\
\hline 11. 2 & 11.6 & 11.9 & 12.1 & 12.3 & 12.5 & 12.6 & 12. 6 & 12.6 \\
\hline $102_{0} \times 10^{-6}$ & $105_{0} \times 10^{-6}$ & $108_{0} \times 10^{-6}$ & $111_{0} \times 10^{-6}$ & $113_{0} \times 10^{-6}$ & $114_{0} \times 10^{-6}$ & $114_{0} \times 10^{-6}$ & $114_{0} \times 10^{-6}$ & $114_{0} \times 10^{-6}$ \\
\hline 863 & 892 & 918 & 940 & 958 & 971 & 980 & 986 & 988 \\
\hline 746 & 771 & 792 & 810 & 825 & 837 & 846 & 852 & 854 \\
\hline 656 & 678 & 696 & 711 & 722 & 730 & 736 & 741 & 744 \\
\hline 580 & 598 & 613 & 625 & 634 & 640 & 644 & 646 & 647 \\
\hline 511 & 527 & 539 & 548 & 554 & 558 & 560 & 561 & 560 \\
\hline 447 & 460 & 470 & 476 & 480 & 482 & 482 & 481 & 479 \\
\hline 389 & 399 & 406 & 410 & 412 & 412 & 410 & 407 & 402 \\
\hline 336 & 344 & 348 & 350 & 350 & 348 & 343 & 337 & 329 \\
\hline 290 & 295 & 297 & 297 & 294 & 290 & 283 & 273 & 262 \\
\hline 250.6 & 252.6 & 2526 & 250.5 & 246.1 & 239.5 & 230.2 & 218.3 & 203.5 \\
\hline 239.0 & 240.3 & 239.7 & 237.0 & 232.0 & 224.7 & 214.9 & 202.4 & 187.0 \\
\hline 215.7 & 215.8 & 213. 9 & 210.0 & 203.8 & 195. 3 & 184. 3 & 170.7 & 154.2 \\
\hline 164.0 & 161. 4 & 157.0 & 150.5 & 142.0 & 131.1 & 117. 9 & 102.1 & 83.59 \\
\hline 159.5 & 156. 7 & 152.0 & 145.4 & 136.6 & 125.6 & 112.2 & 96. 25 & 77. 58 \\
\hline 116.8 & 112.1 & 105.6 & 97.22 & 86.85 & 74.34 & 59.57 & 42. 39 & 22. 64 \\
\hline 83.95 & 77. 96 & 70. 30 & 60.85 & 49.52 & 36.19 & 20.73 & 3. 011 & -17.11 \\
\hline 58. 40 & 51.55 & 43.14 & 33.06 & 21. 21 & 7. 491 & -8.216 & -26.03 & -46.08 \\
\hline 44. 64 & 37.40 & 28.67 & 18.34 & 6. 314 & -7.497 & -23.21 & -40.93 & -60.78 \\
\hline 38.29 & 30.90 & 22.03 & 11. 61 & -0.457 & -14.28 & -29.95 & -47.58 & -67.30 \\
\hline 22.33 & 14. 60 & 5. 500 & -5.058 & -17.16 & -30.88 & -46.34 & -63.61 & -82.82 \\
\hline 9. 574 & 1. 666 & -7.529 & -18.08 & -30.08 & -43.59 & -58.70 & -75.52 & -94.14 \\
\hline-0.687 & -8.670 & -17.86 & -28.31 & -40.11 & -53.33 & -68.04 & -84.33 & -102.3 \\
\hline-8.984 & -16.97 & -26.08 & -36.37 & -47.92 & -60.79 & -75.06 & -90.81 & -108.1 \\
\hline-15.72 & -23.64 & -32.63 & -42.72 & -53.99 & -66.49 & -80.30 & -95.48 & -112.1 \\
\hline-21.20 & -29.03 & -37.85 & -47.72 & -58.68 & -70.80 & -84.14 & -98.76 & -114.7 \\
\hline-25.67 & -33.38 & -42.03 & -51.65 & -62.30 & -74.03 & -86.91 & -101.0 & -116.3 \\
\hline-29.32 & -36.89 & -45.34 & -54.71 & -65.04 & -76.39 & -88.82 & -102.4 & -117.1 \\
\hline-32.30 & -39.72 & -47.96 & -57.08 & -67.10 & -75.08 & -90.06 & -103.1 & -117.3 \\
\hline-34.72 & -41.99 & -50.03 & -58.89 & -68.60 & -79.22 & -90.78 & -103.3 & -116.9 \\
\hline-36.69 & -43.79 & -51.63 & -60.24 & -69.65 & -79.92 & -91.08 & -103.2 & -116.2 \\
\hline
\end{tabular}


TABLE 16. Values of $\left(-d^{2} Z / d T^{2}\right)_{\rho}$ at integral values of $T$,

\begin{tabular}{|c|c|c|c|c|c|c|}
\hline Temperature & $\rho=1$ & 2 & 3 & 6 & 10 & 20 \\
\hline $16{ }^{\circ} K$ & $\begin{array}{l}{ }^{\circ} K^{-2} \\
11.4 \times 10^{-5}\end{array}$ & $\begin{array}{l}{ }^{\circ} K^{-2} \\
22.6 \times 10^{-5}\end{array}$ & $\begin{array}{l}{ }^{\circ} K^{-2} \\
33.7 \times 10^{-5}\end{array}$ & ${ }^{\circ} K^{-2}$ & ${ }^{\circ} K^{-2}$ & ${ }^{\circ} K^{-2}$ \\
\hline 18 & 7.9 & 15.7 & 23.5 & $47.0 \times 10^{-5}$ & & \\
\hline 20 & 5.5 & 11.0 & 16.5 & 33.0 & $54.0 \times 10^{-5}$ & \\
\hline $22 \ldots \ldots$ & 4.0 & 7.9 & 11.7 & 23.5 & 38.6 & \\
\hline 24 & 3.0 & 5. 9 & 8.8 & 17.4 & 29.2 & $48.5 \times 10^{-5}$ \\
\hline $26 \ldots$ & 2.3 & 4. 6 & 6.8 & 13.5 & 22.7 & 41.0 \\
\hline 28 & 1.8 & 3.6 & 5.4 & 10.7 & 17.8 & 34.3 \\
\hline $30 \ldots$ & 1.4 & 2. 9 & 4.3 & 8.6 & 14.3 & 28.4 \\
\hline 32 & 1.2 & 2.4 & 3.5 & 7.0 & 11.7 & 23.3 \\
\hline $34 \ldots . .$. & 0.96 & 2.0 & 2.9 & 5.8 & 9.7 & 19.0 \\
\hline $36 \ldots$ & .79 & 1.7 & 2.4 & 4. 8 & 8.0 & 15.5 \\
\hline $38 \ldots \ldots$. & .66 & 1.4 & 2.0 & 4. 0 & 6.6 & 12.8 \\
\hline $40 \ldots \ldots \ldots$ & .55 & 1.1 & 1.7 & 3.3 & 5.5 & 10.7 \\
\hline \multirow[t]{2}{*}{$42 \ldots$} & .47 & 0.92 & 1.4 & 2.8 & 4. 6 & 9.2 \\
\hline & 220 & 240 & 260 & 280 & 300 & 320 \\
\hline $34 \ldots$ & $167 \times 10^{-5}$ & $178 \times 10^{-5}$ & $186 \times 10^{-5}$ & $190 \times 10^{-5}$ & $190 \times 10^{-5}$ & $188 \times 10^{-5}$ \\
\hline $36 \ldots$ & 138 & 146 & 154 & 160 & 162 & 161 \\
\hline $38 \ldots$ & 114 & 121 & 128 & 135 & 138 & 138 \\
\hline 40 & 96 & 102 & 108 & 114 & 118 & 119 \\
\hline \multirow[t]{3}{*}{42} & 82 & 87 & 92 & 97 & 101 & 103 \\
\hline & $\rho=1$ & 2 & 3 & 6 & 10 & 20 \\
\hline & ${ }^{\circ} K^{-2}$ & ${ }^{\circ} K^{-2}$ & ${ }^{\circ} K^{-2}$ & ${ }^{\circ} K^{-2}$ & ${ }^{\circ} K^{-2}$ & ${ }^{\circ} K^{-2}$ \\
\hline $42 \ldots$ & $0.47 \times 10^{-5}$ & $0.92 \times 10^{-5}$ & 1. $4 \times 10^{-5}$ & $2.8 \times 10^{-5}$ & $4.6 \times 10^{-5}$ & 9. $2 \times 10^{-5}$ \\
\hline $44 \ldots$ & .40 & .79 & 1. 2 & 2.4 & 4.0 & 8.0 \\
\hline $46 \ldots$ & .35 & .69 & 1. 0 & 2.1 & 3.5 & 6. 9 \\
\hline $48 \ldots$ & .31 & .61 & 0.91 & 1.9 & 3.1 & 6.0 \\
\hline $50 \ldots$ & .27 & .53 & .80 & 1.6 & 2.7 & 5. 2 \\
\hline $52 \ldots$ & .23 & .46 & .69 & 1.4 & 2.3 & 4.5 \\
\hline $54 \ldots .$. & .20 & .40 & .60 & 1.2 & 2.0 & 4. 0 \\
\hline $56 \ldots$ & .18 & .35 & .53 & 1.1 & 1.8 & 3. 6 \\
\hline $58 \ldots$. & .16 & .32 & .48 & 0.96 & 1.7 & 3.3 \\
\hline 60 & .15 & .30 & .45 & .90 & 1.5 & 3.0 \\
\hline $65 \ldots$ & .12 & .24 & .37 & .74 & 1. 2 & 2.4 \\
\hline $70 \ldots$ & .10 & .19 & .30 & .60 & 0.96 & 1.9 \\
\hline 75 & .078 & .15 & .23 & .48 & .76 & 1.5 \\
\hline $80 \ldots$ & .063 & .12 & .18 & .38 & .61 & 1.2 \\
\hline $85 \ldots$ & .051 & .10 & .15 & .31 & .49 & 0.97 \\
\hline $90 \ldots \ldots$ & .042 & .084 & .13 & .25 & .41 & .80 \\
\hline $95 \ldots$ & .036 & .070 & .11 & .21 & .35 & .67 \\
\hline $100 \ldots \ldots$ & .030 & 059 & .091 & .18 & .29 & .57 \\
\hline $105 \ldots$. & .025 & .049 & .075 & .15 & .24 & .48 \\
\hline 110 & .020 & .041 & .062 & .12 & .20 & .40 \\
\hline 115 & .017 & .035 & .052 & .10 & .17 & .34 \\
\hline 120 & .015 & .030 & .045 & .089 & .15 & .30 \\
\hline 125 & .013 & .026 & .039 & .080 & .13 & .26 \\
\hline 130 & .012 & .023 & .035 & .071 & .12 & .23 \\
\hline 135 & .011 & .021 & .032 & .062 & .11 & .21 \\
\hline $140 \ldots$ & .0095 & .019 & .028 & .055 & .093 & .19 \\
\hline 145 & .0082 & .016 & .024 & .048 & .081 & .16 \\
\hline 150 & .0071 & .014 & .021 & .043 & .070 & .14 \\
\hline $155 \ldots$ & .0063 & .013 & .019 & .039 & .062 & .13 \\
\hline 160 & .0058 & .012 & .018 & .036 & .057 & .12 \\
\hline 165 & .0054 & .012 & .017 & .033 & .054 & .12 \\
\hline 170 & .0050 & .011 & .016 & .031 & .050 & .11 \\
\hline
\end{tabular}


the absolute temperature, and $\rho$, the density in Amagat units

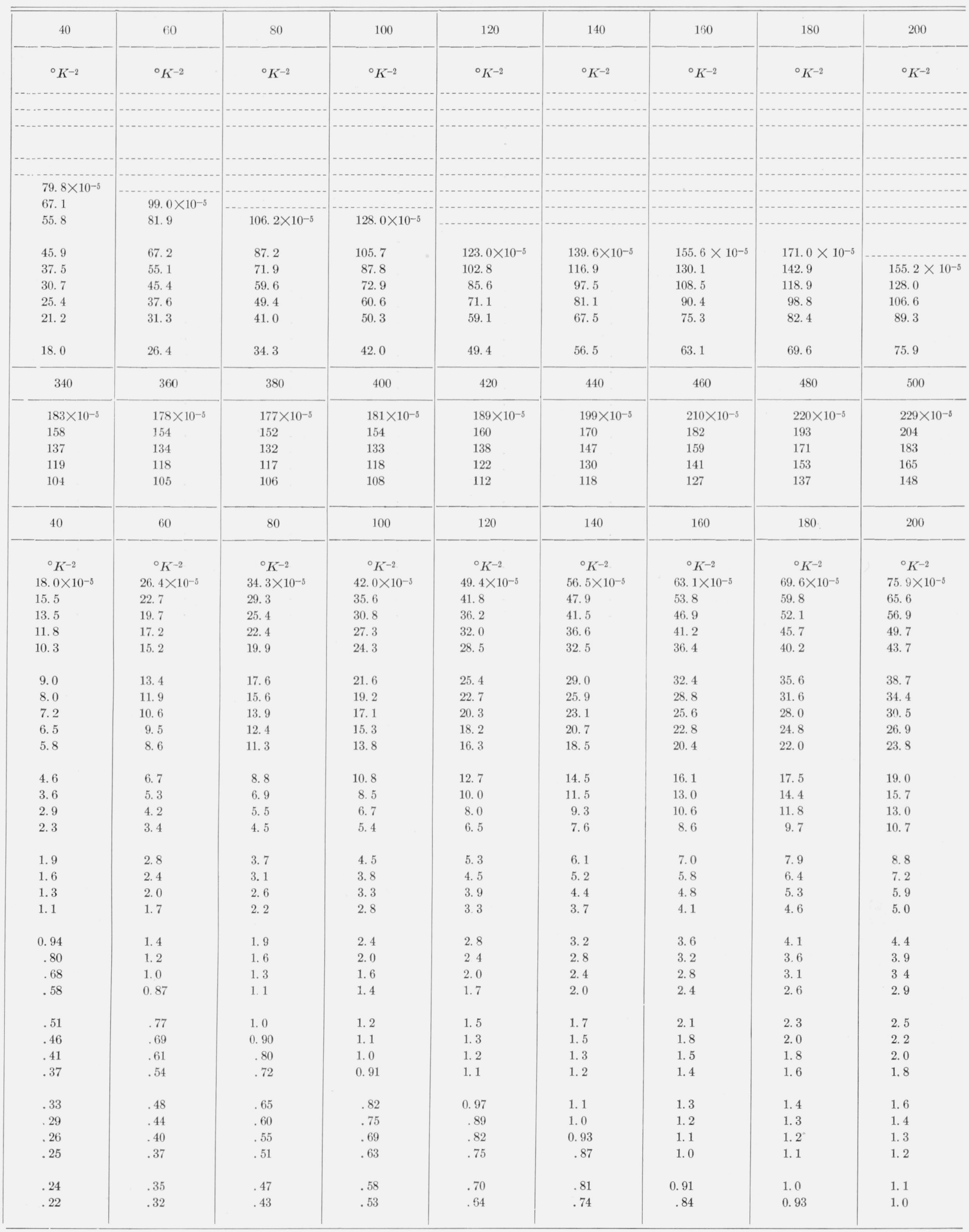


TABLE 16. Values of $\left(-d^{2} \boldsymbol{Z} / d T^{2}\right)_{\rho}$ at integral values of $T$, the

\begin{tabular}{|c|c|c|c|c|c|c|}
\hline Temperature & $\rho=1$ & 2 & 3 & 6 & 10 & 20 \\
\hline 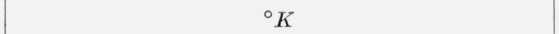 & ${ }^{\circ} K^{-2}$ & ${ }^{\circ} K^{-2}$ & ${ }^{\circ} K^{-2}$ & ${ }^{\circ} \boldsymbol{K}^{-2}$ & ${ }^{\circ} K^{-2}$ & ${ }^{\circ} K^{-2}$ \\
\hline 170 & $0.50 \times 10^{-7}$ & 1. $1 \times 10^{-7}$ & 1. $6 \times 10^{-7}$ & $3.1 \times 10^{-7}$ & $5.0 \times 10^{-7}$ & $11 \times 10^{-r}$ \\
\hline 180 & .44 & 0.88 & 1.3 & 2.6 & 4.3 & 9.3 \\
\hline 190 & .38 & .76 & 1.1 & 2.2 & 3.7 & 7.5 \\
\hline 200 & .33 & .66 & 1.0 & 2.0 & 3.2 & 6.2 \\
\hline 210 & .29 & .58 & 0.87 & 1.8 & 2. 7 & 5.4 \\
\hline 220 & .26 & .51 & .78 & 1.6 & 2.4 & 4.7 \\
\hline 230 & .23 & .45 & .69 & 1.4 & 2. 2 & 4. 2 \\
\hline 240 & .20 & .40 & .60 & 1.2 & 2.0 & 3.7 \\
\hline 250 & .17 & .34 & .52 & 1.0 & 1. 7 & 3. 2 \\
\hline 260 & .14 & .28 & .43 & 0.86 & 1.4 & 2.8 \\
\hline 270 & .1185 & .2370 & .3554 & .7101 & 1. 182 & 2. 356 \\
\hline $0^{\circ} C_{-}$ & .1140 & .2280 & .3419 & .6830 & 1. 137 & 2. 271 \\
\hline 280 & .10501 & .2100 & .3148 & .6289 & 1. 047 & 2. 086 \\
\hline $25^{\circ} \mathrm{C}$ & .08510 & .1701 & .2551 & .5094 & 0.8476 & 1. 691 \\
\hline 300 & .08336 & .1666 & .2498 & .4990 & .8302 & 1. 653 \\
\hline 320 & .06706 & .1341 & .2010 & .4013 & .6674 & 1. 328 \\
\hline 340 & .05459 & 1091 & .1636 & .3266 & .5430 & 1. 079 \\
\hline 360 & .04490 & .08970 & .1345 & .2685 & .4463 & 0.8866 \\
\hline $100^{\circ} C_{\ldots} \ldots$ & .03969 & .07932 & .1189 & .2573 & .3945 & .7837 \\
\hline 380 & .03727 & .07448 & .1116 & .2228 & .3702 & .7350 \\
\hline 400 & .03119 & .06233 & .09342 & .1864 & .3097 & .6143 \\
\hline 420 & .02629 & .05254 & .07875 & .1571 & .2609 & .5173 \\
\hline 440 & .02231 & .04458 & .06682 & .1333 & .2213 & .4385 \\
\hline 460 & .01905 & .03806 & .05703 & $.113 i$ & .1888 & .3739 \\
\hline 480 & .01635 & $.03266^{\circ}$ & .04894 & .09758 & .1620 & .3205 \\
\hline 500 & .01410 & .02817 & .04221 & .08414 & .1396 & .2761 \\
\hline 520 & .01221 & .02440 & .03660 & .07286 & .1209 & .2389 \\
\hline 540 & .01062 & .02122 & .03180 & .06336 & .1051 & .2076 \\
\hline 560 & .06928 & .01853 & .02776 & .05530 & .09169 & .1810 \\
\hline 580 & .00812 & .01623 & .02431 & .04842 & .08027 & .1583 \\
\hline \multirow{2}{*}{600} & .10714 & .01426 & .02136 & .04254 & .07049 & .1390 \\
\hline & $\rho=220$ & 240 & 260 & 280 & 300 & 320 \\
\hline 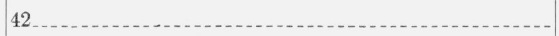 & $82 \times 10^{-5}$ & $87 \times 10^{-5}$ & $92 \times 10^{-5}$ & $97 \times 10^{-5}$ & $101 \times 10^{-5}$ & $103 \times 10^{-5}$ \\
\hline 44 & 71 & 75 & 79 & 84 & 87 & 90 \\
\hline 46 & 61 & 65 & ○9 & 73 & 76 & 80 \\
\hline 48 & 53 & 57 & 61 & 65 & 68 & 72 \\
\hline 50. & 47 & 50 & 54 & 58 & 61 & 64 \\
\hline $52 \ldots$ & 42 & 44 & 47 & 51 & 54 & 57 \\
\hline 54 & 37 & 39 & 41 & 44 & 46 & 49 \\
\hline 56 & 33 & 35 & 36 & 38 & 40 & 42 \\
\hline 58 & 29 & 31 & 32 & 34 & 36 & 37 \\
\hline 60 & 26 & 28 & 30 & 31 & 32 & 34 \\
\hline 65 & 21 & 23 & 25 & 26 & 27 & 28 \\
\hline 70 & 17 & 19 & 21 & 22 & 23 & 24 \\
\hline 75 & 14 & 16 & 17 & 18 & 19 & 20 \\
\hline 80 & 12 & 13 & 14 & 15 & 16 & 16 \\
\hline 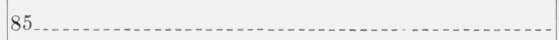 & 9.7 & 10 & 11 & 12 & 13 & 13 \\
\hline 90 & 7.8 & 8.3 & 8.9 & 9.5 & 10 & 11 \\
\hline 95 & 6.4 & 6.9 & 7.5 & 8. 1 & 8.6 & 9.1 \\
\hline $100 \ldots$ & 5.5 & 6.0 & 6.5 & 7.0 & 7.5 & 8.0 \\
\hline 105 & 4.8 & 5.3 & 5.7 & 6.1 & 6.5 & 6.9 \\
\hline 110 & 4. 3 & 4. 6 & 5. 0 & 5. 3 & 5.6 & 6.0 \\
\hline 115 & 3.7 & 4.0 & 4.3 & 4. 6 & 4.9 & 5. 2 \\
\hline 120 & 3.2 & 3.5 & 3.8 & 4. 0 & 4.3 & 4.5 \\
\hline 125 & 2.8 & 3.1 & 3.3 & 3.5 & 3.7 & 3.8 \\
\hline 130 & 2.5 & 2.7 & 2.9 & 3.1 & 3.2 & 3.3 \\
\hline 135 & 2. 2 & 2.4 & 2. 6 & 2.7 & 2.9 & 3.0 \\
\hline 140 & 1.9 & 2.1 & 2.3 & 2.4 & 2.6 & 2.7 \\
\hline
\end{tabular}


absolute temperature, and $\rho$, the density in Amagat units-Continued

\begin{tabular}{|c|c|c|c|c|c|c|c|c|}
\hline 40 & 60 & 80 & 100 & 120 & 140 & 160 & 180 & 200 \\
\hline${ }^{\circ} K^{-2}$ & ${ }^{\circ} K^{-2}$ & ${ }^{\circ} K^{-2}$ & ${ }^{\circ} K^{-2}$ & ${ }^{\circ} K^{-2}$ & ${ }^{\circ} K^{-2}$ & ${ }^{\circ} K^{-2}$ & ${ }^{\circ} K^{-2}$ & ${ }^{\circ} K^{-2}$ \\
\hline $22 \times 10^{-7}$ & $32 \times 10^{-7}$ & $43 \times 10^{-7}$ & $53 \times 10^{-7}$ & $64 \times 10^{-7}$ & $74 \times 10^{-7}$ & $84 \times 10^{-7}$ & $93 \times 10^{-7}$ & $100 \times 10^{-7}$ \\
\hline 19 & 27 & 35 & 44 & 53 & 61 & 70 & 79 & 87 \\
\hline 15 & 22 & 29 & 36 & 43 & 50 & 58 & 65 & 72 \\
\hline 12 & 18 & 24 & 29 & 35 & 41 & 47 & 53 & 59 \\
\hline 10 & 15 & 20 & 25 & 29 & 34 & 39 & 44 & 48 \\
\hline 9.6 & 14 & 18 & 22 & 26 & 29 & 33 & 37 & 41 \\
\hline 8.5 & 13 & 17 & 20 & 23 & 26 & 29 & 32 & 36 \\
\hline 7.5 & 11 & 15 & 18 & 20 & 23 & 26 & 29 & 32 \\
\hline 6.5 & 9.1 & 13 & 16 & 18 & 20 & 23 & 26 & 29 \\
\hline 5.5 & 8.0 & 10 & 13 & 16 & 18 & 20 & 23 & 26 \\
\hline 4. 684 & 6.986 & 9. 264 & 11.52 & 13.76 & 15.82 & 18.19 & 20.38 & 22.56 \\
\hline 4. 523 & $6.75 b$ & 8.970 & 11.17 & 13.35 & 15.51 & 17.66 & 19.79 & 21.91 \\
\hline 4. 142 & b. 171 & 8. 175 & 10.15 & 12.11 & 14.05 & 15. 96 & 17.86 & 19. 74 \\
\hline 3.358 & 5.001 & 6.620 & 8.216 & 9.786 & 11.33 & 12.85 & 14. 35 & 15.81 \\
\hline 3. 276 & 4.872 & b. 440 & 7.981 & 9.496 & 10.98 & 12.45 & 13.89 & 15.30 \\
\hline 2.628 & 3.900 & 5. 145 & 6.362 & 7.553 & 8. 716 & 9.851 & 10.96 & 12.03 \\
\hline 2. 132 & 3.160 & 4. 160 & 5.135 & 6.082 & i. 602 & 7.893 & 8. 756 & 9.588 \\
\hline 1.749 & 2. 587 & 3.400 & 4. 189 & 4. 951 & 5. 687 & 6.395 & 7. 075 & 7.725 \\
\hline 1. 546 & 2. 286 & 3.004 & 3.697 & 4. 366 & 5. 008 & 5.624 & 6. 211 & 6.768 \\
\hline 1. 448 & 2. 138 & 2.806 & 3.450 & 4. 069 & 4. 664 & 5. 232 & 5. 773 & 6. 285 \\
\hline 1. 208 & 1.706 & 2. 202 & 2.865 & 3.373 & 3.857 & 4. 317 & 4. 751 & 5. 158 \\
\hline 1.016 & 1. 496 & 1. 957 & 2.397 & 2.816 & 3.214 & 3.586 & 3. 939 & 4. 265 \\
\hline 0.8602 & 1. 265 & 1. 651 & 2. 019 & 2. 368 & 2. 696 & 3.004 & 3. 289 & 3.550 \\
\hline .7325 & 1.075 & 1. 402 & 1.711 & 2.003 & 2. 276 & 2.529 & 2. 762 & 2.973 \\
\hline .6271 & 0. 9193 & 1. 196 & 1. 458 & 1. 703 & 1. 931 & 2.141 & 2. 332 & 2.503 \\
\hline .5395 & .7897 & 1. 026 & 1. 248 & 1. 455 & 1. 646 & 1.821 & 1. 978 & 2.116 \\
\hline .4662 & .6814 & 0.8839 & 1. 073 & 1. 249 & 1. 410 & 1.555 & 1. 685 & 1.797 \\
\hline .4045 & .5903 & $.76 \dot{4} 45$ & 0.9265 & 1. 076 & 1. 212 & 1. 333 & 1. 440 & 1. 531 \\
\hline .3522 & .5132 & .6635 & .8026 & 0.9299 & 1.045 & 1. 147 & 1. 235 & 1. 309 \\
\hline .3077 & .4476 & $.577 i$ & .6975 & .8063 & 0.9038 & 0.9892 & 1. 062 & 1.121 \\
\hline .2696 & .3916 & .5045 & .6078 & .7011 & .7838 & .8554 & 0.9152 & 0.9626 \\
\hline 340 & 360 & 380 & 400 & 420 & 440 & 460 & 480 & 500 \\
\hline $104 \times 10^{-5}$ & $105 \times 10^{-5}$ & $106 \times 10^{-5}$ & $108 \times 10^{-5}$ & $112 \times 10^{-5}$ & $118 \times 10^{-5}$ & $127 \times 10^{-5}$ & $137 \times 10^{-5}$ & $148 \times 10^{-5}$ \\
\hline 93 & 95 & 98 & 101 & 104 & 109 & 115 & 123 & 132 \\
\hline 84 & 87 & 91 & 95 & 98 & 102 & 106 & 111 & 116 \\
\hline 76 & 80 & 84 & 88 & 92 & 95 & 97 & 99 & 101 \\
\hline 68 & 73 & 77 & 81 & 85 & 87 & 87 & 87 & 88 \\
\hline 61 & 65 & 69 & 73 & 76 & 77 & 77 & 77 & 76 \\
\hline 53 & 57 & 61 & 64 & 66 & 67 & 67 & 67 & 65 \\
\hline 45 & 48 & 51 & 54 & 56 & 56 & 57 & 57 & 56 \\
\hline 39 & 40 & 42 & 45 & 47 & 47 & 48 & 49 & 49 \\
\hline 36 & 36 & 37 & 39 & 41 & 42 & 44 & 45 & 46 \\
\hline 29 & 30 & 31 & 32 & 33 & 34 & 36 & 38 & 39 \\
\hline 25 & 26 & 27 & 28 & 29 & 30 & 31 & 33 & 34 \\
\hline 21 & 22 & 23 & 24 & 25 & 26 & 27 & 29 & 30 \\
\hline 17 & 18 & 19 & 20 & 22 & 23 & 23 & 24 & 25 \\
\hline 14 & 15 & 16 & 17 & 18 & 19 & 19 & 20 & 20 \\
\hline 11 & 12 & 13 & 14 & 14 & 15 & 15 & 16 & 16 \\
\hline 9.6 & 10 & 10 & 11 & 11 & 12 & 12 & 13 & 13 \\
\hline 8. 4 & 8.8 & 9.1 & 9.5 & 9.8 & 10 & 11 & 11 & 11 \\
\hline 7.3 & 7. 7 & 8.0 & 8.4 & 8.7 & 9.1 & 9.4 & 10 & 10 \\
\hline 6.4 & 6.7 & 7.0 & 7.3 & 7. 6 & 8.0 & 8.3 & 8.6 & 8.9 \\
\hline 5.5 & 5.8 & 6.0 & 6.3 & 6.6 & 6.9 & 7. 3 & 7.6 & 7.9 \\
\hline 4. 7 & 4. 9 & 5. 2 & 5. 5 & 5.8 & 6.0 & 6.3 & 6.6 & 6.9 \\
\hline 4. 0 & 4. 2 & 4.4 & 4.8 & 5.1 & 5.3 & 5.5 & 5.8 & 6.0 \\
\hline 3.5 & 3.7 & 4.0 & 4. 2 & 4. 4 & 4. 6 & 4.8 & 5.0 & 5. 2 \\
\hline 3. 2 & 3.4 & 3.6 & 3.7 & 3.9 & 4.0 & 4. 2 & 4.4 & 4.5 \\
\hline 2.9 & 3.1 & 3.2 & 3.3 & 3.4 & 3.6 & 3.7 & 3.8 & 3.9 \\
\hline
\end{tabular}


TABLE 16. Values of $\left(-d^{2} \boldsymbol{Z} / d T^{2}\right)_{\rho}$ at integral values of $T$, the

\begin{tabular}{|c|c|c|c|c|c|c|}
\hline Temperature & $\rho=220$ & 240 & 260 & 280 & 300 & 320 \\
\hline 1. & ${ }^{\circ} K^{-2}$ & ${ }^{\circ} K^{-2}$ & ${ }^{\circ} K^{-2}$ & ${ }^{\circ} K^{-2}$ & ${ }^{\circ} K^{-2}$ & ${ }^{\circ} K^{-2}$ \\
\hline 145 & $\begin{array}{l}1.7 \times 10^{-5} \\
1.5\end{array}$ & $\begin{array}{l}1.8 \times 10^{-5} \\
1.6\end{array}$ & $\begin{array}{l}2.0 \times 10^{-5} \\
1.8\end{array}$ & $\begin{array}{l}2.1 \times 10^{-5} \\
1.9\end{array}$ & $\begin{array}{l}2.3 \times 10^{-5} \\
2.1\end{array}$ & $\begin{array}{l}2.4 \times 10^{-5} \\
2.2\end{array}$ \\
\hline 155 & 1.4 & 1.5 & 1.6 & 1.8 & 1.9 & 2.0 \\
\hline 160 & 1.3 & 1.4 & 1.5 & 1.7 & 1.8 & 1.9 \\
\hline 165 & 1.3 & 1.4 & 1.5 & 1.6 & 1.6 & 1.8 \\
\hline 170 & $110 \times 10^{-7}$ & $120 \times 10^{-7}$ & $130 \times 10^{-7}$ & $140 \times 10^{-7}$ & $150 \times 10^{-7}$ & $160 \times 10^{-7}$ \\
\hline 180 & 96 & 100 & 110 & 120 & 120 & 130 \\
\hline 190 & 78 & 83 & 87 & 91 & 96 & 99 \\
\hline $200 \ldots$ & 63 & 67 & 70 & 73 & 78 & 81 \\
\hline 210 & 52 & 56 & 59 & 63 & 66 & 69 \\
\hline 220 & 45 & 49 & 53 & 56 & 59 & 62 \\
\hline $230 \ldots \ldots$ & 40 & 44 & 48 & 51 & 53 & 56 \\
\hline $240 \ldots$ & 36 & 40 & 43 & 46 & 48 & 51 \\
\hline $250 \ldots$ & 32 & 35 & 38 & 40 & 43 & 46 \\
\hline $260 \ldots$ & 29 & 31 & 33 & 35 & 37 & 40 \\
\hline 270 & 24.73 & 26.89 & 29. 03 & 31.17 & 33.30 & 35.42 \\
\hline $0^{\circ} C_{-}$ & 24. 01 & 26. 09 & 28.15 & 30.20 & 32.23 & 34.24 \\
\hline 280 & 21.60 & 23. 44 & 25.26 & 27.07 & 28.85 & 30.62 \\
\hline $25^{\circ} C_{-}$ & 17. 25 & 18.66 & 20.04 & 21.38 & 22.69 & 23.96 \\
\hline 300 & 16. 68 & 18. 04 & 19. 37 & 20.67 & 21. 94 & 23.17 \\
\hline $320 \ldots$ & 15. 08 & 14. 09 & 15. 08 & 16.02 & 16. 93 & 17.80 \\
\hline $340 \ldots \ldots$ & 10.39 & 11. 16 & 11.89 & 12.58 & 13. 24 & 13.91 \\
\hline $360 \ldots$ & 8.343 & 8. 929 & 9. 480 & 9.994 & 10. 47 & 10.90 \\
\hline $100^{\circ} C_{-}$ & 7. 293 & 7. 785 & 8. 242 & 8. 662 & 9. 043 & 9.383 \\
\hline $380 \ldots$ & 6.767 & 7. 218 & 7. 635 & 8.017 & 8. 360 & 8. 064 \\
\hline 400 & 5. 536 & 5. 885 & 6. 202 & 6.485 & 6. 733 & 6.942 \\
\hline $420_{\ldots}$ & 4. 564 & 4. 834 & 5. 076 & 5. 285 & 5. 461 & 5.601 \\
\hline 440 & 3. 787 & 3. 998 & 4. 181 & 4. 335 & 4. 458 & 4. 547 \\
\hline $460 \ldots$ & 3. 161 & 3.325 & 3. 464 & 3.575 & 3.658 & 3. 709 \\
\hline $480 \ldots$ & 2.653 & 2. 780 & 2. 884 & 2.962 & 3. 014 & 3. 038 \\
\hline $500 \ldots$ & 2. 236 & 2. 334 & 2. 411 & 2.464 & 2. 493 & 2. 496 \\
\hline $520 \ldots \ldots$ & 1.892 & 1.967 & 2. 022 & 2.056 & 2. 067 & 2.055 \\
\hline $540 \ldots$ & 1.606 & 1. 603 & 1. 701 & 1.720 & 1. 718 & 1.693 \\
\hline $560 \ldots$ & 1.367 & 1. 409 & 1. 434 & 1.441 & 1.429 & 1.396 \\
\hline 580 & 1. 166 & 1. 196 & 1. 211 & 1.208 & 1. 188 & 1.149 \\
\hline $600 \ldots \ldots$. & 0.997 & 1.018 & 1.023 & 1.014 & 0.988 & 0.944 \\
\hline
\end{tabular}

TABLE 17. Values of $(d Z / d \rho)_{T}$ at integral values of $T$, the

\begin{tabular}{|c|c|c|c|c|c|c|c|}
\hline Temperature & $\rho=0$ & 1 & 2 & 3 & 6 & 10 & 20 \\
\hline${ }^{\circ} K$ & $-9.105 \times 10^{-6}$ & $-9087 \times 10^{-6}$ & $-9.070 \times 10^{-6}$ & $-9052 \times 10^{-6}$ & & & \\
\hline 18 & $-7,709$ & $-7,694$ & $-7,679$ & $-7,664$ & $-7,620 \times 10^{-6}$ & & \\
\hline $20 \ldots \ldots$ & $-6,633$ & $-6,621$ & $-6,608$ & $-6,595$ & $-6,557$ & $-6,506 \times 10^{-6}$ & \\
\hline $22 \ldots$ & $-5,781$ & $-5,770$ & $-5,759$ & $-5,748$ & $-5,714$ & $-5,670$ & \\
\hline 24 & $-5,087$ & $-5,077$ & $-5,067$ & $-5,058$ & $-5,029$ & $-4,990$ & $-4,892 \times 10^{-6}$ \\
\hline 26 & $-4,512$ & $-4,503$ & $-4,494$ & $-4,485$ & $-4,460$ & $-4,425$ & $-4,338$ \\
\hline $28 \ldots \ldots$ & $-4,027$ & $-4,019$ & $-4,011$ & $-4,003$ & $-3,980$ & $-3,949$ & $-3,871$ \\
\hline $30 \ldots$ & $-3,615$ & $-3,608$ & $-3,601$ & $-3,594$ & $-3,572$ & $-3,544$ & $-3,474$ \\
\hline 32 & $-3,262$ & $-3,255$ & $-3,249$ & $-3,242$ & $-3,223$ & $-3,197$ & $-3,132$ \\
\hline 34 & $-2,955$ & $-2,949$ & $-2,943$ & $-2,937$ & $-2,919$ & $-2,895$ & $-2,836$ \\
\hline 36 & $-2,688$ & $-2,682$ & $-2,676$ & $-2,671$ & $-2,654$ & $-2,632$ & $-2,577$ \\
\hline $38 \ldots$ & $-2,453$ & $-2,448$ & $-2,443$ & $-2,438$ & $-2,422$ & $-2,402$ & $-2,350$ \\
\hline $40 \ldots \ldots$ & $-2,245$ & $-2,240$ & $-2,235$ & $-2,230$ & $-2,216$ & $-2,197$ & $-2,149$ \\
\hline 42 & $-2,059$ & $-2,054$ & $-2,050$ & $-2,045$ & $-2,032$ & $-2,014$ & $-1,968$ \\
\hline
\end{tabular}


absolute temperature, and $\rho$, the density in Amagat units-Continued

\begin{tabular}{|c|c|c|c|c|c|c|c|c|}
\hline 340 & 360 & 380 & 400 & 420 & 440 & 460 & 480 & 500 \\
\hline${ }^{\circ} K_{-2} 2$ & ${ }^{\circ} K^{-2}$ & ${ }^{\circ} K^{-2}$ & ${ }^{\circ} K^{-2}$ & ${ }^{\circ} K^{-2}$ & ${ }^{\circ} K^{-2}$ & ${ }^{\circ} K^{-2}$ & ${ }^{\circ} K^{-2}$ & ${ }^{\circ} K^{-2}$ \\
\hline 2. $6 \times 10^{-5}$ & $2.8 \times 10^{-5}$ & 2. $9 \times 10^{-5}$ & $3.0 \times 10^{-5}$ & $3.1 \times 10^{-5}$ & $3.3 \times 10^{-5}$ & $3.4 \times 10^{-5}$ & $3.4 \times 10^{-5}$ & $3.5 \times 10^{-5}$ \\
\hline 2.3 & 2.5 & 2.6 & 2.7 & 2.9 & 3.0 & 3.2 & 3.2 & 3.3 \\
\hline 2. 1 & 2.2 & 2.3 & 2.5 & 2.7 & 2.8 & 3.0 & 3.1 & 3.2 \\
\hline 2.0 & 2.1 & 2. 2 & 2.4 & 2.5 & 2.6 & 2.7 & 2.9 & 3.0 \\
\hline 2. 0 & 2.1 & 2. 2 & 2.3 & 2.4 & 2.5 & 2.5 & 2.6 & 2. 7 \\
\hline $170 \times 10^{-7}$ & $180 \times 10^{-7}$ & $180 \times 10^{-7}$ & $190 \times 10^{-7}$ & $190 \times 10^{-7}$ & $200 \times 10^{-7}$ & $200 \times 10^{-7}$ & $200 \times 10^{-7}$ & $210 \times 10^{-7}$ \\
\hline 140 & 140 & 140 & 150 & 150 & 150 & 150 & 150 & 160 \\
\hline 100 & 100 & 110 & 110 & 120 & 120 & 120 & 120 & 120 \\
\hline 83 & 85 & 88 & 92 & 96 & 98 & 99 & 100 & 100 \\
\hline 72 & 75 & 78 & 81 & 83 & 85 & 87 & 88 & 89 \\
\hline 65 & 68 & 71 & 73 & 75 & 77 & 80 & 81 & 81 \\
\hline 60 & 63 & 65 & 67 & 69 & 71 & 74 & 75 & 75 \\
\hline 55 & 58 & 60 & 62 & 64 & 66 & 68 & 69 & 70 \\
\hline 49 & 52 & 54 & 56 & 58 & 60 & 62 & 64 & 65 \\
\hline 43 & 46 & 48 & 50 & 52 & 54 & 56 & 58 & 60 \\
\hline 37.53 & 39.63 & 41. 71 & 43.78 & 45.83 & 47.86 & 49. 87 & 51.85 & 53.80 \\
\hline 36.23 & 38.19 & 40.13 & 42.05 & 43.93 & 45.78 & 47.60 & 49. 38 & 51.12 \\
\hline 32.36 & 34.08 & 35.77 & 37.44 & 39.07 & 40. 66 & 42. 22 & 43. 73 & 45. 19 \\
\hline 25. 20 & 26.39 & 27.54 & 28.63 & 29.68 & 30.67 & 31.60 & 32.47 & 33.27 \\
\hline 24.37 & 25.53 & 26.64 & 27.71 & 28.74 & 29.70 & 30.61 & 31.45 & 32.22 \\
\hline 18.63 & 19.41 & 20.14 & 20.82 & 21.44 & 21.99 & 22.48 & 22.90 & 23.23 \\
\hline 14.42 & 14. 94 & 15.41 & 15.83 & 16. 18 & 16.47 & 16. 69 & 16.83 & 16.89 \\
\hline 11. 29 & 11.63 & 11. 92 & 12.16 & 12. 33 & 12.44 & 12.49 & 12.46 & 12.34 \\
\hline 9.678 & 9.926 & 10.12 & 10. 27 & 10.36 & 10.39 & 10.36 & 10. 25 & 10.08 \\
\hline 8.925 & 9.139 & 9. 305 & 9.417 & 9. 473 & 9.468 & 9.397 & 9. 256 & 9. 039 \\
\hline 7. 110 & 7. 236 & 7. 314 & 7. 344 & 7. 319 & 7. 238 & 7. 094 & 6.885 & 6.605 \\
\hline 5. 704 & 5. 765 & 5. 784 & 5. 756 & 5. 678 & 5.547 & 5. 359 & 5. 110 & 4. 795 \\
\hline 4. 601 & 4. 618 & 4. 594 & 4. 527 & 4. 415 & 4. 253 & 4. 039 & 3. 769 & 3.438 \\
\hline 3.728 & 3.713 & 3.660 & 3.567 & 3. 433 & 3.253 & 2.038 & 2. 746 & 2.411 \\
\hline 3. 032 & 2. 993 & 2. 976 & 2.812 & 2. 664 & 2.475 & 2. 242 & 1. 961 & 1. 630 \\
\hline 2. 471 & 2. 416 & 2. 331 & 2. 212 & 2. 057 & 1. 864 & 1. 631 & 1. 354 & 1.032 \\
\hline 2. 016 & 1.951 & 1. 857 & 1. 732 & 1. 574 & 1. 382 & 1. 153 & 0.884 & 0.573 \\
\hline 1. 646 & 1.573 & 1. 474 & 1. 346 & 1. 189 & 1.000 & 0.776 & .517 & .220 \\
\hline 1. 342 & 1. 264 & 1. 162 & 1. 035 & 0.880 & 0.695 & .480 & .231 & -.052 \\
\hline 1.090 & 1. 010 & 0.908 & 0.782 & .630 & .452 & .245 & .007 & -.262 \\
\hline 0.883 & 0.801 & .700 & .576 & .428 & .257 & .059 & -.167 & -.422 \\
\hline
\end{tabular}

absolute temperature, and $\rho$, the density in Amagat units

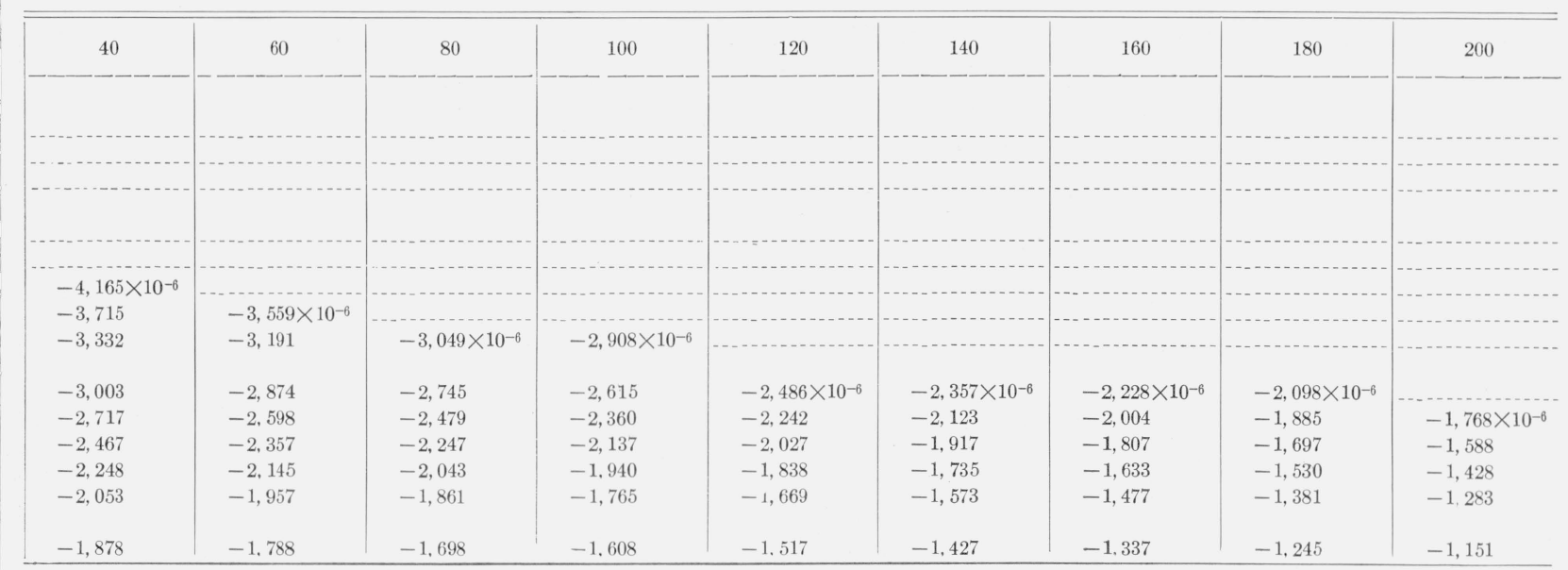


TABLE 17. Values of $(d Z / d \rho,)_{T}$ at integral values of $T$, the absolute

\begin{tabular}{|c|c|c|c|c|c|c|c|}
\hline Temperature & $\rho=220$ & 240 & 260 & 280 & 300 & 320 & 340 \\
\hline $\begin{array}{l}{ }^{\circ} K \\
34 \\
36 \\
38 \\
48\end{array}$ & $\begin{array}{l}-1,650 \times 10^{-6} \\
-1,477 \\
-1,322 \\
-1,181 \\
-1,054\end{array}$ & $\begin{array}{l}-1,526 \times 10^{-6} \\
-1,360 \\
-1,210 \\
-1,077 \\
-956\end{array}$ & $\begin{array}{l}-1,392 \times 10^{-6} \\
-1,239 \\
-1,100 \\
-973 \\
-857\end{array}$ & $\begin{array}{l}-1,254 \times 10^{-6} \\
-1,117 \\
-989 \\
-870 \\
-758\end{array}$ & $\begin{array}{l}-1,121 \times 10^{-6} \\
-1,001 \\
-882 \\
-768 \\
-658\end{array}$ & $\begin{array}{l}-998 \times 10^{-6} \\
-892 \\
-782 \\
-670 \\
-558\end{array}$ & $\begin{array}{l}-884 \times 10^{-6} \\
-788 \\
-684 \\
-573 \\
-461\end{array}$ \\
\hline & $\rho=520$ & 540 & 560 & 580 & 600 & 620 & 640 \\
\hline $\begin{array}{l}34 \ldots \\
36 \\
36_{1} \\
40_{2}\end{array}$ & $\begin{array}{l}387 \times 10^{-6} \\
538 \\
681 \\
812 \\
940\end{array}$ & $\begin{aligned} & 654 \times 10^{-6} \\
& 795 \\
& 961 \\
& 1,135\end{aligned}$ & $\begin{array}{l}\quad 941 \times 10^{-6} \\
1,077 \\
1,260\end{array}$ & $\begin{array}{l}1,255 \times 10^{-6} \\
1,400 \\
1,587\end{array}$ & $\begin{array}{l}1,627 \times 10^{-6} \\
1,750\end{array}$ & $\begin{array}{l}2,090 \times 10^{-6} \\
2,120\end{array}$ & $\begin{array}{l}2,638 \times 10^{-6} \\
2,504\end{array}$ \\
\hline & $\rho=0$ & 1 & 2 & 3 & 6 & 10 & 20 \\
\hline $\begin{array}{l}44^{4} \ldots \\
46 \\
48 \\
48\end{array}$ & $\begin{array}{l}-2,059 \times 10^{-6} \\
-1,892 \\
-1,740 \\
-1,603 \\
-1,478\end{array}$ & $\begin{array}{l}-2,054 \times 10^{-6} \\
-1,887 \\
-1,736 \\
-1,599 \\
-1,474\end{array}$ & $\begin{array}{l}-2,050 \times 10^{-6} \\
-1,883 \\
-1,732 \\
-1,595 \\
-1,470\end{array}$ & $\begin{array}{l}-2,045 \times 10^{-6} \\
-1,879 \\
-1,728 \\
-1,591 \\
-1,467\end{array}$ & $\begin{array}{l}-2,032 \times 10^{-6} \\
-1,866 \\
-1,716 \\
-1,580 \\
-1,456\end{array}$ & $\begin{array}{l}-2,014 \times 10^{-6} \\
-1,849 \\
-1,700 \\
-1,564 \\
-1,441\end{array}$ & $\begin{array}{l}-1,968 \times 10^{-6} \\
-1,806 \\
-1,659 \\
-1,526 \\
-1,404\end{array}$ \\
\hline $\begin{array}{l}52 \\
54 \\
56 \\
58 \\
60\end{array}$ & $\begin{array}{r}-1,364 \\
-1,259 \\
-1,162 \\
-1,072 \\
-988\end{array}$ & $\begin{array}{r}-1,360 \\
-1,256 \\
-1,159 \\
-1,069 \\
-985\end{array}$ & $\begin{array}{r}-1,357 \\
-1,252 \\
-1,156 \\
-1,066 \\
-982\end{array}$ & $\begin{array}{r}-1,353 \\
-1,249 \\
-1,152 \\
-1,063 \\
-979\end{array}$ & $\begin{array}{r}-1,342 \\
-1,239 \\
-1,143 \\
-1,053 \\
-970\end{array}$ & $\begin{array}{r}-1,328 \\
-1,225 \\
-1,129 \\
-1,040 \\
-958\end{array}$ & $\begin{array}{r}-1,293 \\
-1,191 \\
-1,096 \\
-1,009 \\
-928\end{array}$ \\
\hline 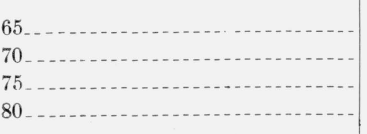 & $\begin{array}{l}-807 \\
-654 \\
-524 \\
-412\end{array}$ & $\begin{array}{l}-804 \\
-651 \\
-522 \\
-410\end{array}$ & $\begin{array}{l}-801 \\
-649 \\
-519 \\
-407\end{array}$ & $\begin{array}{l}-799 \\
-646 \\
-516 \\
-405\end{array}$ & $\begin{array}{l}-790 \\
-638 \\
-509 \\
-398\end{array}$ & $\begin{array}{l}-779 \\
-628 \\
-499 \\
-388\end{array}$ & $\begin{array}{l}-751 \\
-601 \\
-474 \\
-364\end{array}$ \\
\hline 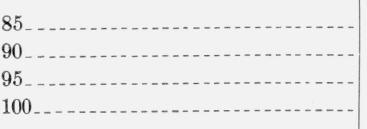 & $\begin{array}{r}-315 \\
-231 \\
-157 \\
-90\end{array}$ & $\begin{array}{r}-313 \\
-229 \\
-155 \\
-88\end{array}$ & $\begin{array}{r}-310 \\
-227 \\
-153 \\
-86\end{array}$ & $\begin{array}{r}-308 \\
-224 \\
-150 \\
-84\end{array}$ & $\begin{array}{r}-301 \\
-218 \\
-144 \\
-77\end{array}$ & $\begin{array}{l}-292 \\
-209 \\
-135 \\
-69\end{array}$ & $\begin{array}{r}-269 \\
-186 \\
-113 \\
-48\end{array}$ \\
\hline 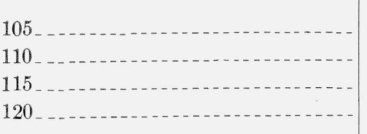 & $\begin{array}{r}-34 \\
+19 \\
67 \\
111\end{array}$ & $\begin{array}{r}-32 \\
+21 \\
69 \\
113\end{array}$ & $\begin{array}{r}-30 \\
+23 \\
71 \\
115\end{array}$ & $\begin{array}{r}-28 \\
+25 \\
73 \\
117\end{array}$ & $\begin{array}{r}-21 \\
+32 \\
79 \\
123\end{array}$ & $\begin{array}{r}-12 \\
+40 \\
87 \\
131\end{array}$ & $\begin{array}{r}+9 \\
61 \\
108 \\
151\end{array}$ \\
\hline 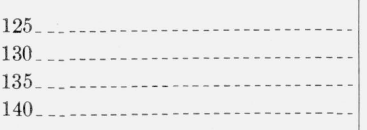 & $\begin{array}{l}151 \\
187 \\
221 \\
252\end{array}$ & $\begin{array}{l}153 \\
189 \\
223 \\
254\end{array}$ & $\begin{array}{l}155 \\
191 \\
225 \\
256\end{array}$ & $\begin{array}{l}157 \\
193 \\
227 \\
258\end{array}$ & $\begin{array}{l}139 \\
199 \\
232 \\
263\end{array}$ & $\begin{array}{l}171 \\
206 \\
240 \\
271\end{array}$ & $\begin{array}{l}190 \\
226 \\
259 \\
289\end{array}$ \\
\hline 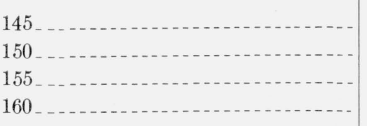 & $\begin{array}{l}281 \\
307 \\
331 \\
353\end{array}$ & $\begin{array}{l}283 \\
309 \\
333 \\
355\end{array}$ & $\begin{array}{l}285 \\
311 \\
335 \\
357\end{array}$ & $\begin{array}{l}286 \\
312 \\
336 \\
358\end{array}$ & $\begin{array}{l}292 \\
318 \\
342 \\
364\end{array}$ & $\begin{array}{l}297 \\
325 \\
349 \\
371\end{array}$ & $\begin{array}{l}317 \\
343 \\
367 \\
389\end{array}$ \\
\hline $\begin{array}{l}165_{1} \ldots \ldots \\
170_{1} \\
180_{1} \\
190 \\
200_{1}\end{array}$ & $\begin{array}{l}373 \\
393 \\
429 \\
460 \\
489\end{array}$ & $\begin{array}{l}375 \\
395 \\
431 \\
462 \\
490\end{array}$ & $\begin{array}{l}377 \\
397 \\
433 \\
463 \\
491\end{array}$ & $\begin{array}{l}378 \\
398 \\
434 \\
465 \\
493\end{array}$ & $\begin{array}{l}384 \\
404 \\
439 \\
470 \\
498\end{array}$ & $\begin{array}{l}391 \\
411 \\
446 \\
477 \\
504\end{array}$ & $\begin{array}{l}409 \\
429 \\
464 \\
494 \\
521\end{array}$ \\
\hline $\begin{array}{l}210 \ldots \\
220_{1} \\
230_{2} \\
240_{1} \\
250_{1}\end{array}$ & $\begin{array}{l}512 \\
534 \\
553 \\
571 \\
587\end{array}$ & $\begin{array}{l}514 \\
536 \\
555 \\
573 \\
589\end{array}$ & $\begin{array}{l}516 \\
538 \\
557 \\
574 \\
590\end{array}$ & $\begin{array}{l}517 \\
539 \\
558 \\
576 \\
592\end{array}$ & $\begin{array}{l}522 \\
544 \\
563 \\
580 \\
596\end{array}$ & $\begin{array}{l}529 \\
550 \\
569 \\
586 \\
602\end{array}$ & $\begin{array}{l}545 \\
566 \\
585 \\
602 \\
617\end{array}$ \\
\hline $\begin{array}{l}260 \ldots+20 \\
270\end{array}$ & $\begin{array}{l}602 \\
613.9\end{array}$ & $\begin{array}{l}604 \\
615.3\end{array}$ & $\begin{array}{l}605 \\
616.8\end{array}$ & $\begin{array}{l}606 \\
618.2\end{array}$ & $\begin{array}{l}610 \\
622.5\end{array}$ & $\begin{array}{l}616 \\
628.3\end{array}$ & $\begin{array}{l}631 \\
642.8\end{array}$ \\
\hline
\end{tabular}


temperature, and $\rho$, the density in Amagat units-Continued

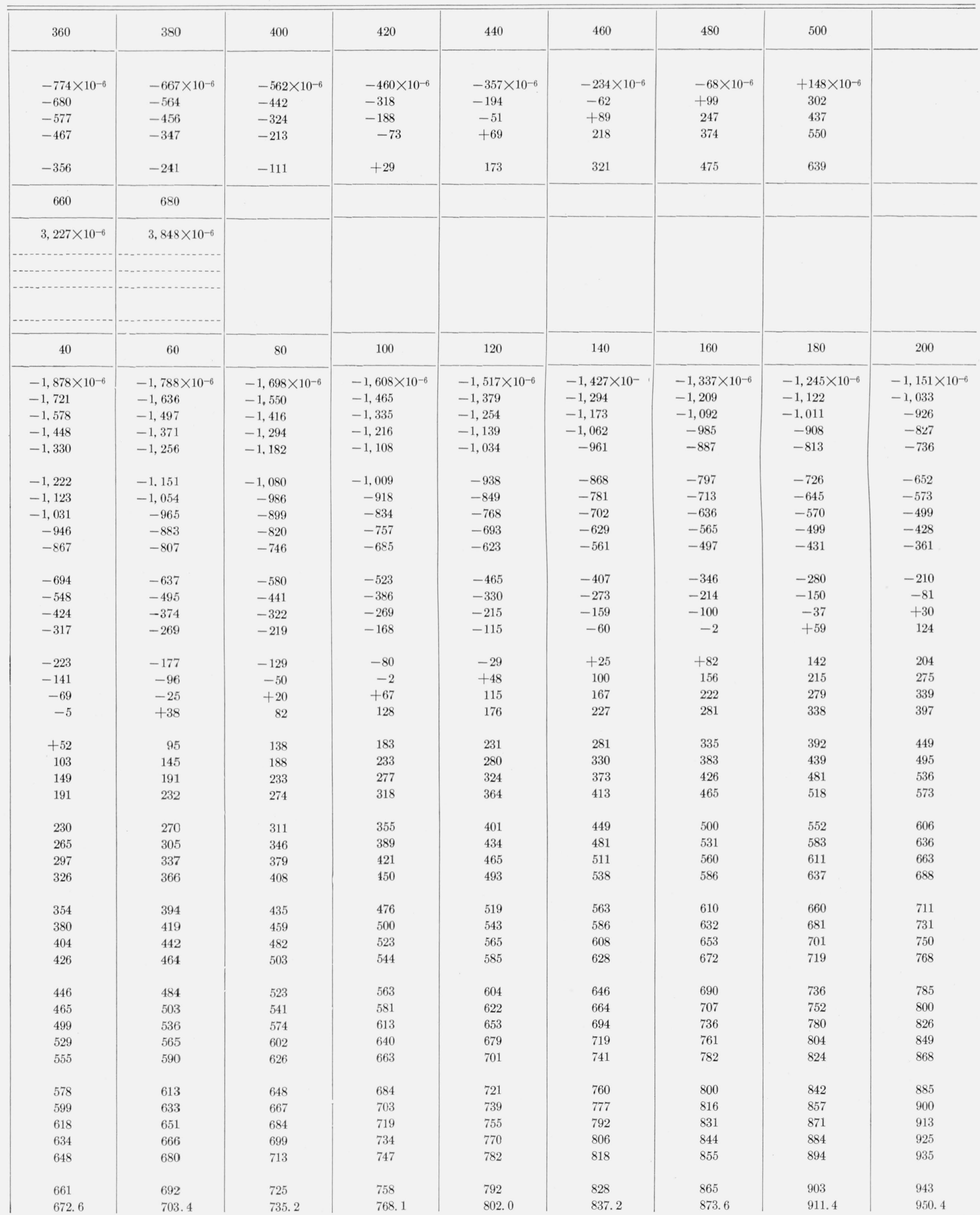


TABLE 17. Values of $\left(d Z / d_{\rho}\right)_{T}$ at integral values of $T$, the

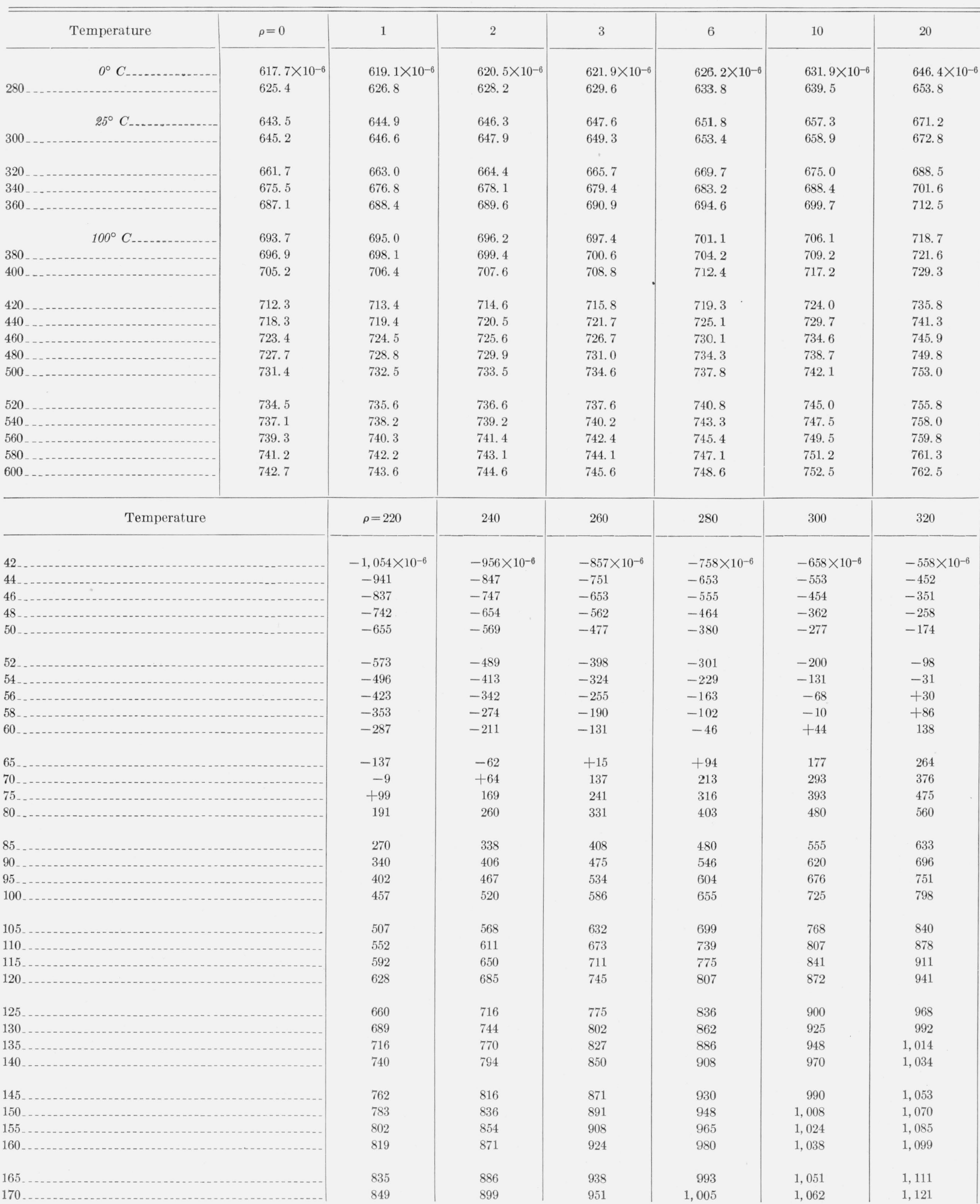


absolute temperature, and $\rho$, the density in Amagat units-Continued

\begin{tabular}{|c|c|c|c|c|c|c|c|c|}
\hline 40 & 60 & 80 & 100 & 120 & 140 & 160 & 180 & 200 \\
\hline $\begin{array}{l}676.1 \times 10^{-6} \\
683.2\end{array}$ & $\begin{array}{l}706.7 \times 10^{-6} \\
713.5\end{array}$ & $\begin{array}{l}738.4 \times 10^{-6} \\
744.8\end{array}$ & $\begin{array}{l}771.0 \times 10^{-6} \\
777.1\end{array}$ & $\begin{array}{l}804.9 \times 10^{-6} \\
810.6\end{array}$ & $\begin{array}{l}839.8 \times 10^{-6} \\
845.2\end{array}$ & $\begin{array}{l}876.1 \times 10^{-6} \\
881.1\end{array}$ & $\begin{array}{l}913.6 \times 10^{-6} \\
918.2\end{array}$ & $\begin{array}{l}952.5 \times 10^{-6} \\
956.7\end{array}$ \\
\hline 699.8 & 729.2 & 759.7 & 791. 2 & 823.7 & 857.4 & 892.3 & 928.4 & 965.8 \\
\hline 701.3 & 730.7 & 761.0 & 792.4 & 824.9 & 858.5 & 893.2 & 929.2 & 966.5 \\
\hline 716.2 & 744.7 & 774.2 & 804.7 & 836.2 & 868.8 & 902.5 & 937.4 & 973.5 \\
\hline 728.4 & 756. 2 & 784.8 & 814.5 & 845.1 & 876.7 & 909.5 & 943.3 & 978.4 \\
\hline 738.6 & 765.6 & 793.5 & 822.3 & 852.1 & 882.9 & 914.7 & 947.6 & 981.6 \\
\hline 744.4 & 770.9 & 798.4 & 826.6 & 855.9 & 886.1 & 917.3 & 949.6 & 983.0 \\
\hline 747.1 & 773.4 & 800.6 & 828.6 & 857.6 & 887.6 & 918.5 & 950.5 & 983.6 \\
\hline 754.2 & 779.9 & 806.4 & 833.7 & 861.9 & 891.1 & 921.2 & 952.4 & 984.5 \\
\hline 760.1 & 785.2 & 811.0 & 837.7 & 865.2 & 893.7 & 923.0 & 953.4 & 984.7 \\
\hline 765.1 & 789.5 & 814.8 & 840.8 & 867.7 & 895.5 & 924.1 & 953.7 & 984.2 \\
\hline 769.2 & 793.1 & 817.8 & 843.3 & 869.5 & 896.6 & 924.6 & 953.5 & 983.3 \\
\hline 772.6 & 796.0 & 820.2 & 845.1 & 870.8 & 897.3 & 924.6 & 952.8 & 981.9 \\
\hline 775.4 & 798.3 & 822.0 & 846.4 & 871.6 & 897.5 & 924.2 & 951.8 & 980.2 \\
\hline 777.6 & 800.2 & 823.4 & 847.3 & 871.9 & 897.3 & 923.5 & 950.4 & 978.2 \\
\hline 779.5 & 801.6 & 824.4 & 847.8 & 871.9 & 896.8 & 922.4 & 948.9 & 976.1 \\
\hline 780.9 & 802.6 & 825.0 & 848.0 & 871.7 & 896.1 & 921. 2 & 947.1 & 973.8 \\
\hline 782.1 & 803.4 & 825.3 & 847.9 & 871.2 & 895.1 & 919.8 & 945.2 & 971.3 \\
\hline 782.9 & 803.9 & 825.4 & 847.6 & 870.5 & 894.0 & 918.2 & 943.1 & 968.7 \\
\hline 340 & 360 & 380 & 400 & 420 & 440 & 460 & 480 & 500 \\
\hline$-461 \times 10^{-6}$ & $-356 \times 10^{-6}$ & $-241 \times 10^{-6}$ & $-111 \times 10^{-6}$ & $+29 \times 10^{-6}$ & $173 \times 10^{-6}$ & $321 \times 10^{-6}$ & $475 \times 10^{-6}$ & $639 \times 10^{-6}$ \\
\hline-352 & -249 & -139 & -15 & +120 & 259 & 403 & 553 & 707 \\
\hline-250 & -149 & -42 & +76 & 203 & 335 & 471 & 612 & 759 \\
\hline-157 & -57 & +48 & 162 & 281 & 404 & 531 & 664 & 804 \\
\hline-73 & +27 & 130 & 240 & 353 & 468 & 588 & 715 & 849 \\
\hline+2 & 102 & 204 & 310 & 418 & 528 & 643 & 765 & 894 \\
\hline+68 & 168 & 270 & 373 & 478 & 585 & 697 & 815 & 939 \\
\hline 128 & 228 & 329 & 431 & 534 & 640 & 750 & 864 & 984 \\
\hline 183 & 282 & 382 & 484 & 587 & 692 & 800 & 913 & 1,031 \\
\hline 234 & 332 & 431 & 532 & 635 & 740 & 848 & 961 & 1,079 \\
\hline 355 & 450 & 547 & 647 & 752 & 861 & 973 & 1,089 & 1,210 \\
\hline 464 & 556 & 652 & 751 & 855 & 963 & 1.075 & 1,191 & 1,312 \\
\hline 561 & 651 & 745 & 842 & 944 & 1,050 & 1,160 & 1,274 & 1,394 \\
\hline 644 & 733 & 824 & 919 & 1,018 & 1,122 & 1,231 & 1,345 & 1,465 \\
\hline 715 & 802 & 892 & 984 & 1. 081 & 1,183 & 1,291 & 1,405 & 1,526 \\
\hline 776 & 860 & 948 & 1,039 & 1,134 & 1,234 & 1,340 & 1,454 & i, 576 \\
\hline 829 & 911 & 997 & 1,086 & 1,180 & 1,279 & 1,384 & 1,496 & 1,616 \\
\hline 875 & 956 & 1,041 & 1,129 & 1,221 & 1,319 & 1,422 & 1,531 & 1,646 \\
\hline 916 & 996 & 1,080 & 1,167 & 1,258 & 1,355 & 1,456 & 1,561 & 1,670 \\
\hline 953 & 1,032 & 1,115 & 1,201 & 1,291 & 1,387 & 1,486 & 1,587 & 1,693 \\
\hline 985 & 1,064 & 1,146 & 1,231 & 1,320 & 1,414 & 1,511 & 1,611 & 1,715 \\
\hline 1,014 & 1,092 & 1,173 & 1,257 & 1,345 & 1,437 & 1,533 & 1,633 & 1,736 \\
\hline 1,041 & 1,117 & 1,197 & 1,280 & 1,366 & 1,457 & 1,552 & 1,652 & 1,754 \\
\hline 1,064 & 1,140 & 1,218 & 1,299 & 1. 384 & 1,473 & 1,567 & 1,667 & 1,768 \\
\hline 1,085 & 1,159 & 1,236 & 1,316 & 1,399 & 1,487 & 1,580 & 1,678 & 1,779 \\
\hline 1,103 & 1,176 & 1,252 & 1,331 & 1,413 & 1,500 & 1,591 & 1,686 & 1,785 \\
\hline 1,120 & 1,191 & 1,266 & 1,345 & 1,426 & 1,511 & 1,600 & 1,693 & 1,790 \\
\hline 1,136 & 1,205 & 1,278 & 1,356 & 1,436 & 1,520 & 1,607 & 1,699 & 1,795 \\
\hline 1,150 & 1,218 & 1,290 & 1,366 & 1,444 & 1,526 & 1,612 & 1,704 & 1,799 \\
\hline 1,162 & 1,229 & 1,300 & 1,374 & 1,451 & 1,531 & 1,616 & 1,707 & 1,801 \\
\hline 1,173 & 1,240 & 1,309 & 1,381 & 1,457 & 1,536 & 1,619 & 1,707 & 1,800 \\
\hline 1,183 & 1,248 & 1,317 & 1,388 & 1,462 & 1,539 & 1,620 & 1,706 & 1,797 \\
\hline
\end{tabular}


TABLE 17. Values of $\left(d Z / d_{\rho}\right)_{T}$ at integral values of $T$, the absolute

\begin{tabular}{|c|c|c|c|c|c|c|}
\hline Temperature & $\rho=220$ & 240 & 260 & 280 & 300 & 320 \\
\hline $180 \ldots$ & $874 \times 10^{-6}$ & $922 \times 10^{-6}$ & $972 \times 10^{-6}$ & 1. $025 \times 10^{-6}$ & $1,081 \times 10^{-6}$ & $1,140 \times 10^{-6}$ \\
\hline $190 \ldots$ & 895 & 943 & 992 & 1,043 & 1,098 & 1,156 \\
\hline $200 \ldots$ & 913 & 961 & 1,009 & 1,060 & 1,113 & 1,169 \\
\hline $210 \ldots$ & 929 & 976 & 1,024 & 1,074 & 1,126 & 1,180 \\
\hline 220 & 943 & 989 & 1,037 & 1,086 & 1,137 & 1,190 \\
\hline $230 \ldots$ & 956 & 1,001 & 1,048 & 1,096 & 1,146 & 1,198 \\
\hline $240 \ldots$ & 967 & 1,011 & 1.057 & 1,104 & 1,154 & 1,205 \\
\hline 250 & 976 & 1,020 & 1,065 & 1,111 & 1,160 & 1,211 \\
\hline $260 \ldots$. & 984 & 1,027 & 1,072 & 1,117 & 1,165 & 1,215 \\
\hline 270 & 991.0 & $1,033.1$ & $1,076.8$ & $1,122.1$ & $1,169.3$ & $1,218.3$ \\
\hline $0^{\circ} C_{2}$ & 992.9 & $1,034.7$ & $1,078.2$ & $1,123.3$ & $1,170.2$ & $1,219.0$ \\
\hline $280 \ldots \ldots$ & 996.6 & $1,038,0$ & $1,081.0$ & $1,125.6$ & $1,172.0$ & $1,220.2$ \\
\hline $25^{\circ} C_{-}$ & $1,004.5$ & $1,044.7$ & $1,086.4$ & $1,129.7$ & $1,174.7$ & $1,221.4$ \\
\hline 300 & $1,005.2$ & $1,045.3$ & $1,086.8$ & $1,130.0$ & $1,174.8$ & $1,221.4$ \\
\hline $320 \ldots$ & 1,011. 0 & $1,049.8$ & $1,090.1$ & $1,131.8$ & $1,175.1$ & $1,220.1$ \\
\hline $340 \ldots$ & $1,014.7$ & $1,052.3$ & $1,091.3$ & $1,131.7$ & $1,173.6$ & $1,217.1$ \\
\hline 360 & $1,016.9$ & $1,053.4$ & 1,091.2 & $1,130.3$ & $1,170.9$ & $1,212.9$ \\
\hline $100^{\circ} C_{-}$ & $1,017.6$ & $1,053,4$ & 1.090 .4 & $1,128.8$ & $1,168.5$ & $1,209.7$ \\
\hline $380 \ldots .$. & $1,017.8$ & $1,053.2$ & $1,089.9$ & $1,127.9$ & $1,167.2$ & $1,207.9$ \\
\hline 400 & $1,017.8$ & $1,052.2$ & $1,087.8$ & $1,124.7$ & $1,162.9$ & $1,202.4$ \\
\hline $420 \ldots$ & $1,017.1$ & $1,050.6$ & $1,085.2$ & $1,121.0$ & $1,158.0$ & $1,196.4$ \\
\hline $440 \ldots$ & $1,015.8$ & $1,048.4$ & $1,082.1$ & $1,116.9$ & $1,152.9$ & $1,190.2$ \\
\hline 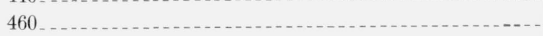 & $1,014.0$ & $1,045.8$ & $1,078.6$ & $1,112.6$ & $1,147.6$ & $1,183.8$ \\
\hline 480 & 1,011. 9 & $1,042.9$ & $1,074.9$ & $1,108.0$ & $1,142.1$ & $1,177.4$ \\
\hline 500 & $1,009.5$ & $1,039.8$ & $1,071.0$ & $1,103.3$ & $1,136.6$ & $1,171.0$ \\
\hline $520 \ldots$ & $1,006.9$ & $1,036.5$ & $1,067.0$ & $1,098.5$ & $1,131.0$ & $1,164.6$ \\
\hline (n) & $1,004.2$ & $1,033.1$ & $1,062.9$ & $1,093.7$ & $1,125.4$ & $1,158.2$ \\
\hline 560 & $1,001.2$ & $1,029.6$ & $1,058.7$ & $1,088.8$ & $1,119.9$ & $1,151.9$ \\
\hline 580 & 998.2 & $1,026.0$ & $1,054.6$ & $1,084.0$ & $1,114.4$ & $1,145.7$ \\
\hline 600 & 995.1 & $1,022.3$ & $1,050.3$ & $1,079.2$ & $1,108.9$ & $1,139.5$ \\
\hline
\end{tabular}


temperature, and $\rho$, the density in Amagat units-Continued

\begin{tabular}{|c|c|c|c|c|c|c|c|c|}
\hline 340 & 360 & 380 & 400 & 420 & 440 & 460 & 480 & 400 \\
\hline $\begin{array}{l}1,201 \times 10^{-6} \\
1,215 \\
1,227\end{array}$ & $\begin{array}{l}1,264 \times 10^{-6} \\
1,276 \\
1,287\end{array}$ & $\begin{array}{l}1,330 \times 10^{-6} \\
1,341 \\
1,350\end{array}$ & $\begin{array}{l}1,399 \times 10^{-6} \\
1,408 \\
1,416\end{array}$ & $\begin{array}{l}1,471 \times 10^{-6} \\
1,478 \\
1,484\end{array}$ & $\begin{array}{l}1,545 \times 10^{-6} \\
1,550 \\
1,550\end{array}$ & $\begin{array}{l}1,623 \times 10^{-6} \\
1,626 \\
1,629\end{array}$ & $\begin{array}{l}1,706 \times 10^{-6} \\
1,707 \\
1,708\end{array}$ & $\begin{array}{l}1,794 \times 10^{-6} \\
1,792 \\
1,790\end{array}$ \\
\hline $\begin{array}{l}1,237 \\
1,246 \\
1,253 \\
1,259 \\
1,264\end{array}$ & $\begin{array}{l}1,296 \\
1,304 \\
1,310 \\
1,315 \\
1,319\end{array}$ & $\begin{array}{l}1,358 \\
1,365 \\
1,370 \\
1,373 \\
1,376\end{array}$ & $\begin{array}{l}1,422 \\
1,428 \\
1,432 \\
1,434 \\
1,436\end{array}$ & $\begin{array}{l}1,489 \\
1,493 \\
1,496 \\
1,497 \\
1,498\end{array}$ & $\begin{array}{l}1,559 \\
1,561 \\
1.562 \\
1,563 \\
1,563\end{array}$ & $\begin{array}{l}1,632 \\
1,633 \\
1,633 \\
1,633 \\
1,631\end{array}$ & $\begin{array}{l}1,708 \\
1,708 \\
1,708 \\
1,706 \\
1,702\end{array}$ & $\begin{array}{l}1,788 \\
1,787 \\
1,786 \\
1,783 \\
1,778\end{array}$ \\
\hline $\begin{array}{l}1,267 \\
1,269.3 \\
1,269.7 \\
1,270.3 \\
1,269.9 \\
1,269.7\end{array}$ & $\begin{array}{l}1,321 \\
1,322.4 \\
1,322.5 \\
1,322.4 \\
1,320.3 \\
1,320.0\end{array}$ & $\begin{array}{l}1,378 \\
1,377.6 \\
1,377.4 \\
1,376.6 \\
1,372.8 \\
1.372 .3\end{array}$ & $\begin{array}{l}1,436 \\
1,435.2 \\
1,434.6 \\
1,433.1 \\
1,427.3 \\
1,426.6\end{array}$ & $\begin{array}{l}1,497 \\
1,495.1 \\
1,494.2 \\
1,491.9 \\
1,484.1 \\
1,483.2\end{array}$ & $\begin{array}{l}1,561 \\
1,557.6 \\
1,556.3 \\
1,553.2 \\
1,543.3 \\
1,542.2\end{array}$ & $\begin{array}{l}1,628 \\
1,622.8 \\
1,621.1 \\
1,617.1 \\
1,604.8 \\
1,603.5\end{array}$ & $\begin{array}{l}1,697 \\
1,690.8 \\
1,688.6 \\
1,683.7 \\
1,669.0 \\
1,667.4\end{array}$ & $\begin{array}{l}1,771 \\
1,761.8 \\
1,759.1 \\
1,753.1 \\
1,735.8 \\
1,734.0\end{array}$ \\
\hline $\begin{array}{l}1,266.8 \\
1,262.2 \\
1,256.6 \\
1,252.4 \\
1,250.2 \\
1.243 .3\end{array}$ & $\begin{array}{l}1,315.3 \\
1,309.0 \\
1,301.8 \\
1,296.7 \\
1,293.9 \\
1,285.6\end{array}$ & $\begin{array}{l}1,365.7 \\
1,357.7 \\
1,348.8 \\
1,342.6 \\
1.339 .3 \\
1,329.6\end{array}$ & $\begin{array}{l}1,418.1 \\
1,408.2 \\
1,397.5 \\
1,390.2 \\
1,386.4 \\
1,375.1\end{array}$ & $\begin{array}{l}1,472.5 \\
1,460.6 \\
1,448.0 \\
1,439.6 \\
1,435.2 \\
1,422.3\end{array}$ & $\begin{array}{l}1,529.2 \\
1,515.1 \\
1,500.6 \\
1,490.9 \\
1,485.8 \\
1,471.2\end{array}$ & $\begin{array}{l}1,588.1 \\
1,571.8 \\
1,555.1 \\
1,544.1 \\
1,538.4 \\
1,521.9\end{array}$ & $\begin{array}{l}1,649.5 \\
1,630.8 \\
1,611.8 \\
1,599.4 \\
1,593.0 \\
1,574.6\end{array}$ & $\begin{array}{l}1,713.4 \\
1,692.0 \\
1,670.7 \\
1,656.8 \\
1.649 .7 \\
1,629.2\end{array}$ \\
\hline $\begin{array}{l}1,236.1 \\
1,228.7 \\
1,221.3 \\
1,213.9 \\
1,206.5\end{array}$ & $\begin{array}{l}1,277.2 \\
1,268.6 \\
1,260.1 \\
1,251.6 \\
1,243.2\end{array}$ & $\begin{array}{l}1,319.7 \\
1,309.9 \\
1,300.1 \\
1,290.5 \\
1,281.1\end{array}$ & $\begin{array}{l}1,363.8 \\
1,352.6 \\
1,341.6 \\
1,330.8 \\
1,320.2\end{array}$ & $\begin{array}{l}1,409.4 \\
1,396.8 \\
1,384.5 \\
1,372.4 \\
1,360.7\end{array}$ & $\begin{array}{l}1,456.7 \\
1,442.6 \\
1,428.9 \\
1,415.5 \\
1,402.6\end{array}$ & $\begin{array}{l}1,505.8 \\
1,490.0 \\
1,474.8 \\
1,460.1 \\
1,445.8\end{array}$ & $\begin{array}{l}1,556.6 \\
1,539.2 \\
1,522.4 \\
1,506.2 \\
1,490.6\end{array}$ & $\begin{array}{l}1,609.3 \\
1,590.1 \\
1,571.6 \\
1,553.9 \\
1.536 .9\end{array}$ \\
\hline $\begin{array}{l}1,199.2 \\
1,192.0 \\
1,184.9 \\
1,177.9 \\
1,171.1\end{array}$ & $\begin{array}{l}1,234.9 \\
1,226.8 \\
1,218.9 \\
1,211.2 \\
1,203.6\end{array}$ & $\begin{array}{l}1,271.8 \\
1,262.8 \\
1,254.0 \\
1,245.5 \\
1,237.2\end{array}$ & $\begin{array}{l}1,310.0 \\
1.300 .0 \\
1,290.3 \\
1,280.9 \\
1.271 .7\end{array}$ & $\begin{array}{l}1,349.4 \\
1,338.4 \\
1,327.7 \\
1,317.4 \\
1,307.4\end{array}$ & $\begin{array}{l}1,390.1 \\
1,378.0 \\
1,366.3 \\
1,355.0 \\
1,344.1\end{array}$ & $\begin{array}{l}1,432.1 \\
1,418.9 \\
1,406.2 \\
1,393.9 \\
1,382.0\end{array}$ & $\begin{array}{l}1,475.6 \\
1,461.2 \\
1,447.3 \\
1,434.0 \\
1,421.2\end{array}$ & $\begin{array}{l}1,520.5 \\
1,504.8 \\
1,489.8 \\
1,475.4 \\
1,461.5\end{array}$ \\
\hline
\end{tabular}


Many thermodynamic equations involve derivatives in which $P, V$, and $T$ are the variables of state. Applications of the tables of this paper in which the variables are $Z, \rho$, and $T$ to calculations of properties involving derivatives in which the variables are $P, V$, and $T$ may be facilitated by means of equations relating the $P, V, T$ and the $\mathrm{Z}, \rho, T$ derivatives. The following are adequate for many ordinary uses:

$$
\begin{gathered}
\frac{T}{P}\left(\frac{d P}{d T}\right)_{V}=\frac{T}{P}\left(\frac{d P}{d T}\right)_{\rho}=\frac{T}{P}\left(\frac{d S}{d V}\right)_{T}=1+\frac{T}{Z}\left(\frac{d Z}{d T}\right)_{\rho}{ }_{(4.2)} \\
-\frac{V}{P}\left(\frac{d P}{d V}\right)_{T}=\frac{\rho}{P}\left(\frac{d P}{d \rho}\right)_{T}=1+\frac{\rho}{Z}\left(\frac{d Z}{d \rho}\right)_{T}(4.3) \\
-\frac{T}{V}\left(\frac{d V}{d T}\right)_{P}=\frac{T}{\rho}\left(\frac{d \rho}{d T}\right)_{P}=\frac{T}{V}\left(\frac{d S}{d P}\right)_{T}:-\frac{1+\frac{T}{Z}\left(\frac{d Z}{d T}\right)_{\rho}}{1+\frac{\rho}{Z}\left(\frac{d Z}{d \rho}\right)_{T}} .
\end{gathered}
$$

The Joule-Thomson coefficient $\mu$ may be utilized to illustrate the use of these formulas. Thus for purposes of calculations with the tables of this paper, the familiar equation

$$
\mu=\left(\frac{d T}{d P}\right)_{H}=\frac{V}{C_{p}}\left[\frac{T}{V}\left(\frac{d V}{d T}\right)_{P}-1\right],
$$

is put in the form

$$
\mu=\left(\frac{d T}{d P}\right)_{H}=\frac{V_{0}}{\rho C_{p}}\left[\frac{1+\frac{T}{Z}\left(\frac{d Z}{d T}\right)_{\rho}}{1+\frac{\rho}{Z}\left(\frac{d Z}{d \rho}\right)_{T}}-1\right],
$$

where $V_{0}$ is the molar volume of hydrogen at standard conditions and $C_{p}$ is the molar heat capacity at the given conditions of $T$ and $P$ or $T$ and $\rho$.

In correlating the PVT data for hydrogen the function

$$
\sigma=\frac{T}{T_{0}} \frac{V}{V_{0}} \log _{10} \frac{P V}{R T}
$$

was used, where $T_{0}$ is the Kelvin temperature of the ice point. Reported temperatures were reduced wherever possible to a thermodynamic scale having the ice point temperature $273.16^{\circ}$. All available data were considered in this work but only those appearing most reliable were used and these were weighted according to their apparent precision. The data used $[59,61,63,65,66,67$. 70 to $74,76,79,81,85,88,91,177]$ are plotted in figure 6 with the exception of a few observations at temperatures below $29^{\circ} \mathrm{K}$ and at densities lower than $\rho=10$, which were omitted because in these regions of low precision the scattering is so great that the points would be confusing.

A lower boundary to the $\sigma$ versus $\rho$ gas-liquid diagram in figure 6 is furnished by the vaporliquid saturation line and the freezing curve. These are represented in figure 6 by dashed lines. The saturation line for the vapor rises steeply onto the diagram at low densities and with decreasing slope approaches tangency to the critical isotherm at the critical point which is indicated by an asterisk. The saturation line for liquid hydrogen is a nearly straight and horizontal line from a density somewhat greater than the critical to the triple point. The freezing curve, which represents the values of $\sigma$ for liquid when for a given temperature the pressure is great enough to cause the liquid to freeze, rises nearly vertically from the triple point and bends towards higher densities.

The saturation curve on the vapor side was obtained with the help of the vapor pressure equation (eq 7.2) and the PVT representation given by eq 4.14 and table 19 . On the liquid side it was obtained from the same vapor pressure equation and the volumes of the liquid at saturation pressure, given in table 31 and discussed in section VIII. The freezing curve was obtained from the melting point-pressure relations given in table 30 combined with extrapolations based on the higher density observations of Bartholomé for the isotherms of the liquid which are given in table 32 .

The isothermal curves of figure 6 represent final table values. The curves are not necessarily the best fit for the experimental data for each individual isotherm inasmuch as the curves and table values are the result of correlating all the data and include the temperature dependence which, while it does not affect the relative position of points on one isotherm, may shift the whole isotherm somewhat. Isotherms that depended upon only a few individual observations and covered only a small range of densities were given less weight than others. For a given isotherm, data at higher densities, corresponding to larger deviations from the ideal gas law, were usually given 


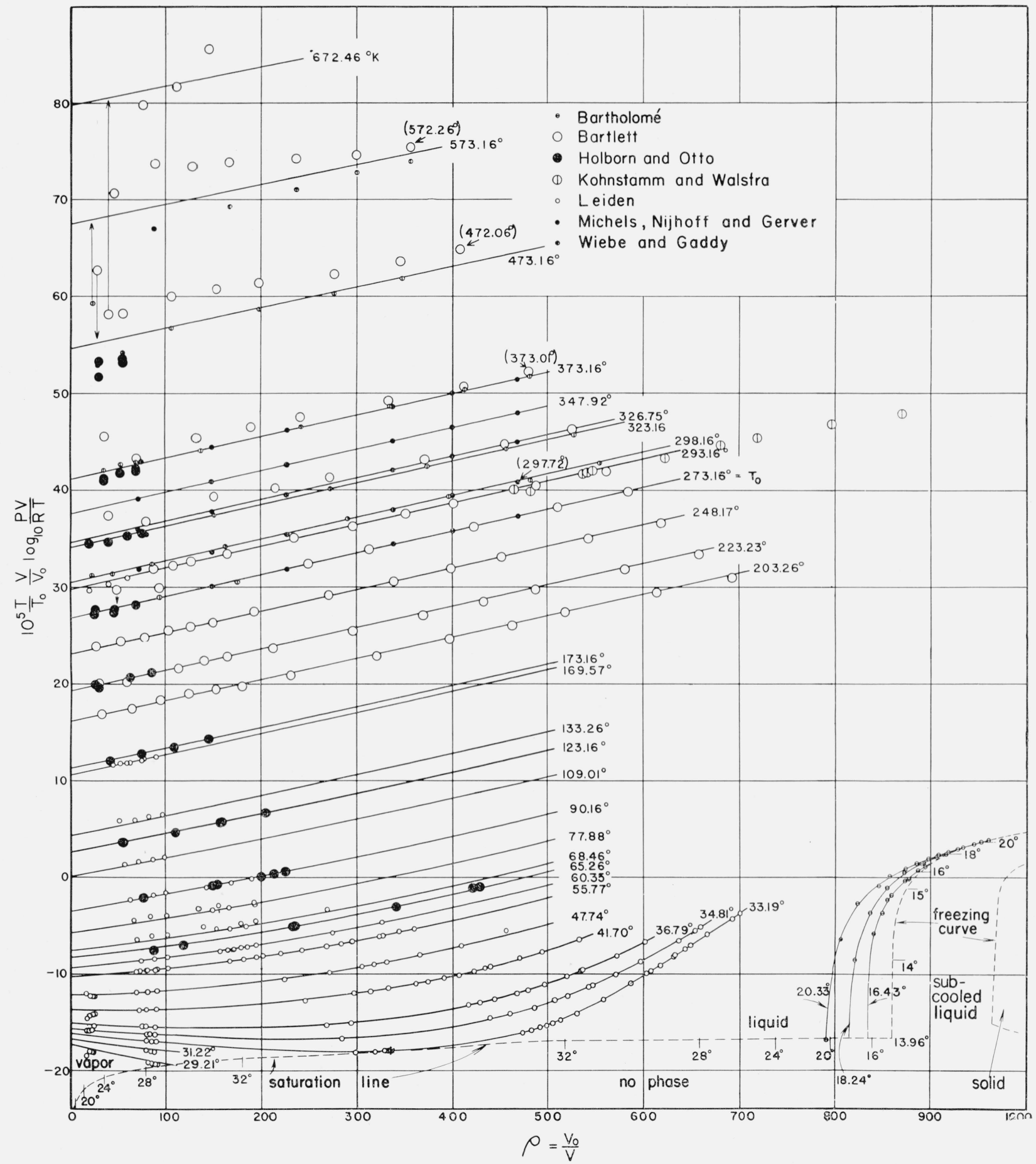

Figure 6. Plot of PVT data for $\mathrm{H}_{2}$ in the fluid states.

greater weight than data at low densities. In fact in some instances the low density data were given zero weight. Data at the highest temperatures do not appear to be very reliable, probably because of penetration of the containers by hydrogen. At very low temperatures the deviations from the ideal gas law have not been measured very precisely because the pressure range over which 
measurements can be made is limited by condensation.

Cragoe has shown that for densities up to $\rho=500$ the $0^{\circ} \mathrm{C}$ isotherm is fitted to within experimental accuracy by the equation $\sigma=b+c \rho$. Figure 6 shows that, although this linear relation between $\sigma$ and $\rho$ fails at low temperatures, it is valid within experimental error over a considerable range of temperatures above $200^{\circ} \mathrm{K}$. This relation was made the basis for the correlation of the PVT data above $0^{\circ} \mathrm{C}$. The different method used for correlating the data below $0^{\circ} \mathrm{C}$ is described under (b).

\section{(a) Region Above $0^{\circ} \mathrm{C}$}

Above $0^{\circ} \mathrm{C}$, equations of the form $\sigma=b+c \rho$ were fitted to the PVT data plotted in figure 6 , and $b$ and $c$, the intercept and slope of an isothermal line, were determined as functions of $T$. The quantity $Z=P V / R T$ thus obtained as a function of $T$ and $\rho$,

$$
\begin{aligned}
P V / R T= & \exp 2.30259 \frac{T_{0}}{T}\left[b(T) \rho+c(T) \rho^{2}\right]= \\
& \exp \left[B(T) \rho+C(T) \rho^{2}\right],
\end{aligned}
$$

was used for the calculation of the tables of $Z$. $P,(d Z / d \rho)_{T},(d Z / d T)_{\rho}$, and $\left(d^{2} Z / d T^{2}\right)_{\rho}$.

Before fitting functions of $T$ to $b$ and $c$, small corrections were applied to some of the data. A constant error in $T$ and constant factor errors along an isotherm in $P, V$, and the number of moles of gas, cause deviations from the true isotherm that are very nearly proportional to $1 / \rho$. Such hyperbolic deviations from a straight line are most easily detected in data extending from low to high densities. A change in $V$ by 0.2 percent is sufficient to considerably straighten the $573.16^{\circ} \mathrm{K}\left(300^{\circ} \mathrm{C}\right)$ isotherm of Wiebe and Gaddy, and raise the line drawn through their adjusted data so that it intersects the $\sigma$ axis of figure 6 only 0.7 unit below the table line for $573.16^{\circ} \mathrm{K}$ and crosses the table line at $\rho=550$. Wiebe and Gaddy call attention in their paper to an estimated error of 0.05 to 0.10 percent in the volume of their high pressure steel pipette at $200^{\circ}$ and $300^{\circ} \mathrm{C}$. It would seem that some part of the 0.2-percent adjustment, which straightens the $300^{\circ} \mathrm{C}$ isotherm of Wiebe and Gaddy, might be attributed to small temperature and pressure errors and to some loss of hydrogen in the steel.

Hyperbolic adjustments proportional to $1 / \rho$ of
Bartlett's higher temperature data straighten the isotherms and improve their agreement with the lines representing the tables. A comparison of the observations of Michels, Nijhoff, and Gerver [79] at different temperatures for nearly constant values of $\rho$, revealed apparent small hyperbolic trends of the data for the separate isotherms superposed on one larger though small random pattern of scattering common to all their isotherms. Using their $0^{\circ} \mathrm{C}$ isotherm as a reference line, their other data were adjusted to remove the hyperbolic deviations. The points of figure 6 represent reported data adjusted only to the Kelvin scale having $273.16^{\circ}$ at the ice point.

Least square determinations were made of the straight lines fitting the adjusted $\sigma$ versus $\rho$ isothermal data for the different observers separately. From these, values of intercept $b$ and slope $c$ were obtained for the different observers at each temperature of measurement. Holborn's data above $0^{\circ} \mathrm{C}$, however, were used only for obtaining intercepts, the slopes of adjacent isotherms of other observers being used with his data.

Expanding the exponential of eq 4.8 ,

$$
\begin{aligned}
& P V / R T=1+B \rho+\left[(1 / 2) B^{2}+C\right] \rho^{2}+\left[(1 / 6) B^{3}+B C\right] \rho^{3} \\
& \quad+\left[(1 / 24) B^{4}+(1 / 2) C^{2}+(1 / 2) B^{2} C\right] \rho^{4}+\ldots \quad(4.9)
\end{aligned}
$$

shows that $E(T)$ is the second virial coefficient and that a correlation of intercepts $b$ of $\sigma$-isotherms is essentially a correlation of values of the second virial coefficients of hydrogen. Formulas expressing the dependence of the second virial coefficient on temperature have been derived theoretically on the assumption of simple laws of intermolecular forces. One of the most satisfactory formulas is based on a law of intermolecular force of the form $\lambda_{n} r^{-n}-\lambda_{m} r^{-m}$ and is due to LennardJones. For $n=13$ and $m=7$, the Lennard-Jones formula for $B$ is

$$
B=B_{1} T^{-1 / 4}+B_{2} T^{-3 / 4}+B_{3} T^{-5 / 4}+\ldots,
$$

where all the coefficients $E_{i}$ of this infinite series are determined by $\lambda_{n}$ and $\lambda_{m}$. Following essentially a procedure used sucessfully by F. G. Keyes [89], we used only the first three terms of this series and selected values for $E_{1}, E_{2}$, and $B_{3}$ which resulted in the best fit of a three constant equation with the intercepts of the $\sigma$-isotherms. Our formula,

$B=0.0055478 T^{-1 / 4}-0.036877 T^{-3 / 4}-0.22004 T^{-5 / 4}$,

Journal of Research 
intended for use above $0^{\circ} \mathrm{C}$, passes through the intercept of the $-50^{\circ} \mathrm{C}$ isotherm determined by the correlation below $0^{\circ} \mathrm{C}$.

The slopes of the $\sigma$-isotherms were represented by a two term empirical formula without theoretical justification, except that it involves powers of $T$ which make $C$ go to zero as $T$ grows very large.

$$
C=0.004788 T^{-3 / 2}-0.04053 T^{-2} .
$$

The exponents of $T$ were chosen so as to simplify the temperature function coefficients in the power series in $\rho$ of eq 4.9 .

The tables from $270^{\circ}$ to $600^{\circ} \mathrm{K}$ have been computed on the basis of these formulas, and in
0.06 percent for the $100^{\circ} \mathrm{C}$ isotherm, and for the other isotherms it is of this approximate magnitude or smaller. At low densities the deviation for the $0^{\circ} \mathrm{C}$ isotherm does not appear to be systematic. On the other hand, it will be seen that there is a systematic deviation at densities greater than 500 with the experimental values for $\sigma$ less than those obtained by linear extrapolation from the intermediate densities. This trend is supported by the high pressure data of Kohnstamm and Walstra [61, 81], also shown in the figure. If the representation of the $\sigma$ isotherm by an equation is extended beyond $\rho=500$, it will be necessary to include a small quadratic term in the expression for $\sigma$.

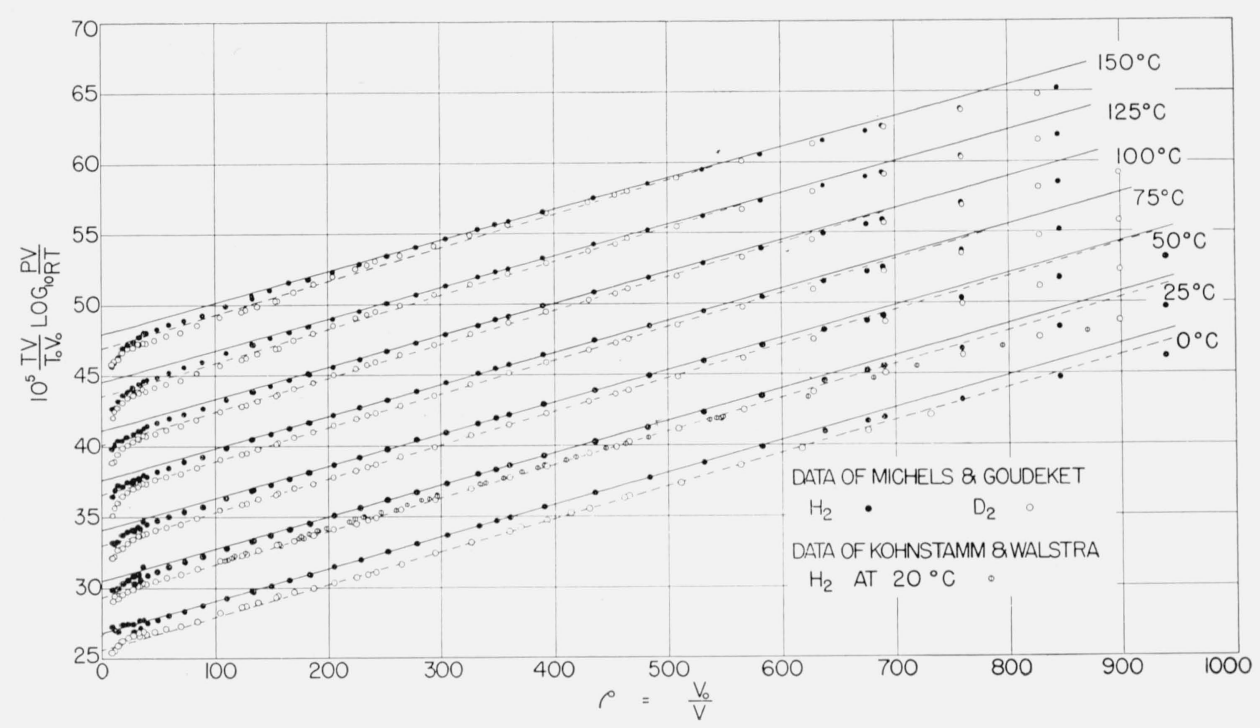

Figure 7. A plot of part of the PVT data for $\mathrm{H}_{2}$ and $\mathrm{D}_{2}$ from $0^{\circ} \mathrm{C}$ to $150^{\circ} \mathrm{C}$.

this temperature range the various derivatives tabulated have been calculated analytically.

It was not until considerably after the preparation of the tables on hydrogen that we were able to examine the data of Michels and Goudeket published in Physica 1941 [91]. Values of $\sigma$ for these data on $\mathrm{H}_{2}$ are shown as solid circles in figure 7 with the tables represented by the solid straight lines. The agreement for $\mathrm{H}_{2}$ is not complete but seems fairly satisfactory at moderate densities. At low densities there are discrepancies, roughly hyperbolic, which have the appearance of the hyperbolic deviations resulting from small systematic errors discussed earlier in this section. If the hyperbolic deviation is attributed to a systematic error in the volume, the error amounts to (b) Region Below $0^{\circ} \mathrm{C}$

At low temperatures the $\sigma$ versus $\rho$ isotherms are curved; making it difficult to decide how the isotherm should be drawn at low densities where the data were meager and the precision was low. Another function, $T^{3 / 2} V / V_{0}\left(1-\frac{P V}{R T}\right)$, plotted against $\rho=V_{0} / V$ as abscissa gave lines which appeared to be straight at low densities for temperatures below $56^{\circ} \mathrm{K}$, though there is considerable curvature at high densities. In figure 8, $T^{3 / 2} V / V_{0}\left(1-\frac{\mathrm{PV}}{R T}\right)+0.0006 \rho=\psi$ is plotted against $\rho$, the term $0.0006 \rho$ being added to make isotherms nearly horizontal at low densities and thus increase the scale of the plot. The sensitivity to 
small changes of $P V / R T$ at $\rho=200$ and $T=55^{\circ} \mathrm{K}$ is 18 times greater in figure 8 than in figure 6 and 14 times greater at $\rho=200$ and $T=33^{\circ} \mathrm{K}$. The curves of figure 8 were drawn to fit the data for each particular isothern considered independently, and though the curves do not represent the tables exactly they agree closely with them. Below $31^{\circ} \mathrm{K}$ the data were not sufficient and precise enough to determine consistent isothermal curves when the isotherms were considered independently. The data lower than $29^{\circ} \mathrm{K}$ were not plotted because the double valued nature of $\psi$ causes the data below $29^{\circ} \mathrm{K}$ to fall in the same region on the diagram as is covered by the data above $29^{\circ} \mathrm{K}$.

At first it appeared that the critical isotherm in figure 8 could be represented by a straight line from $\rho$ equal to zero to $\rho$ greater than the critical density. However, the conditions that $(d P / d V)_{T}$ and $\left(d^{2} P / d V^{2}\right)_{T}$ be zero at the critical point impose upon the slope and curvature of the isotherm at the critical point the conditions

$$
\left.\begin{array}{l}
\left(\frac{d \psi}{d \rho}\right)_{T_{c}}=\frac{T_{c}^{3 / 2}}{\rho_{c}^{2}}\left(2 \frac{P_{c} V_{c}}{R T_{c}}-1\right)+0.0006, \\
\left(\frac{d^{2} \psi}{d \rho^{2}}\right)_{T_{c}}=\frac{2 T_{c}^{3 / 2}}{\rho_{c}^{3}}\left(1-3 \frac{P_{c} V_{c}}{R T_{c}}\right) .
\end{array}\right\}
$$

In addition, values for the critical temperature and pressure should satisfy the vapor-pressure equation.

Only a single determination has been made of the critical temperature and pressure of hydrogen [62]. The critical isotherm was located somewhere between the 2 measured isotherms at $32.94^{\circ} 7$ and $33.29^{\circ} \mathrm{K}$, and was at the time (1917) considered to be $33.19^{\circ} \mathrm{K}$ with a certainty of about $0.1^{\circ}$, though in 1925 it was stated in a footnote to Leiden Communication 172a that $T_{c}$ should be about $0.1^{\circ}$ lower. The critical pressure inferred from the $P$ versus $V$ isotherms in 1917 was 12.80 atm. Later in 1917 [142] the vapor pressure equation of $\mathrm{H}_{2}$ above the boiling point was determined and the value $12.75 \mathrm{~atm}$ deduced for $P_{c}$ using $T_{c}=33.18^{\circ} \mathrm{K}$ (on basis of $T_{0}=273.09$ ). Two determinations [62] were made of the critical density based on the extrapolation of the rectilinear diameter. These gave $\rho_{c}=345$. The values reported in later Leiden Communications have not in all cases been the latest determined values. The most recently reported Leiden values [69] are

${ }^{7}$ Unless otherwise stated, temperatures are expressed on the Kelvin Scale with $\mathrm{T}_{0}=273.16^{\circ}$

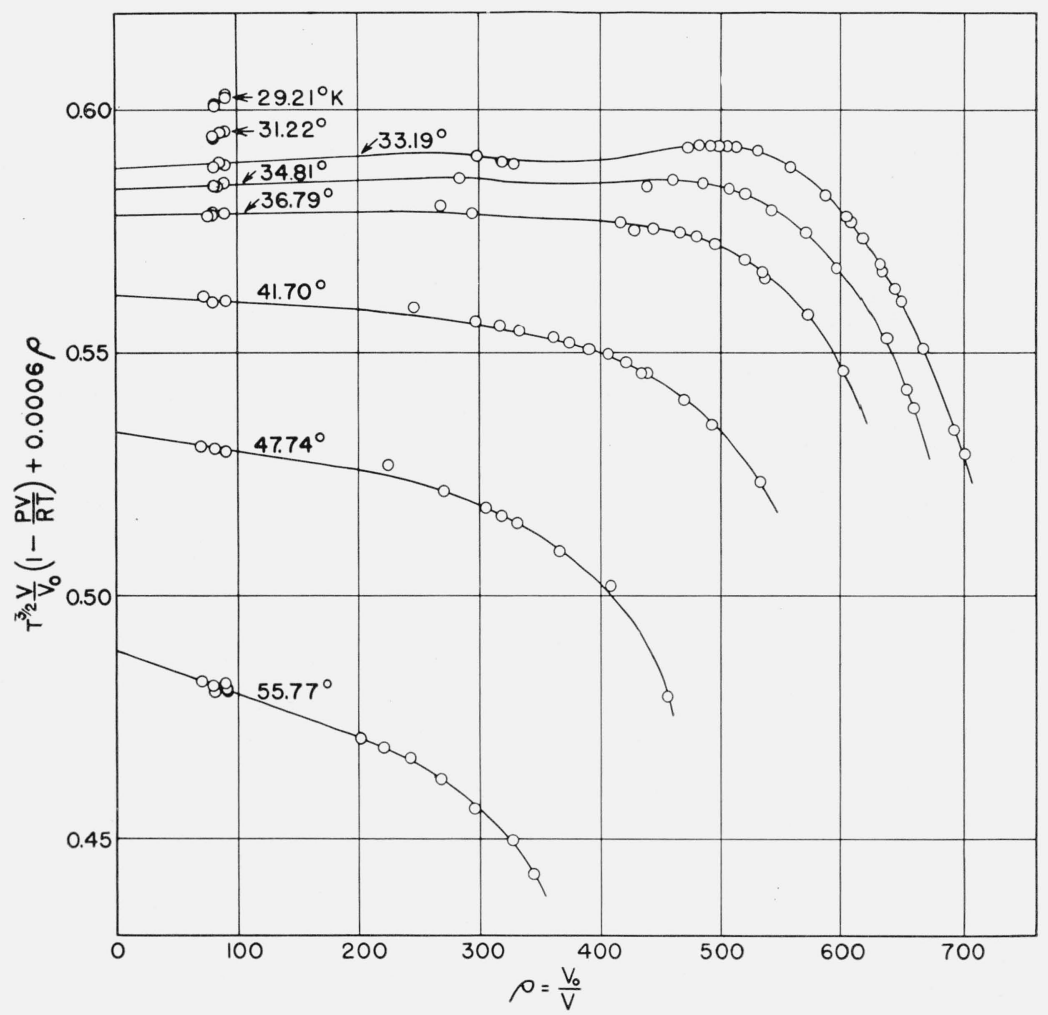

Figure 8. Plot of PVT data for $\mathrm{H}_{2}$ at low temperatures. 
$T_{c}=33.19^{\circ} \mathrm{K}\left(\right.$ on basis of $\left.T_{0}=273.16\right), P_{c}=12.751$ atm and $1 / \rho_{c}=0.02909$ or $\rho_{c}=344$. The lower critical temperature $33.1^{\circ} K$ inferred from Leiden Communication $172 \mathrm{a}$ is supported by the agreement of the vapor pressure $12.81 \mathrm{~atm}$, calculated from vapor pressure equation (eq 7.2) with the critical pressure determined in 1917 from the $P$ versus $V$ isotherms.

Difficulties are encountered in obtaining agreement with the experimental PVT data (fig. 8) vapor pressure equation (7.2). These critical constants are listed in table 18.

TABLE 18. Critical constants of hydrogen

\begin{tabular}{|c|c|c|c|c|}
\hline$T_{c}$ & $P_{c}$ & $\rho_{c}=\frac{V_{0}}{V_{c}}$ & $V_{c}$ & $\frac{P_{c} V_{c}}{R T_{c}}$ \\
\hline${ }^{\circ} K$ & atm & & $c m^{3} m^{2} e_{e}^{-1}$ & \\
33.19 & 12.98 & 335 & 66.95 & 0.3191 \\
\hline
\end{tabular}

It seemed reasonable to assume that the iso-

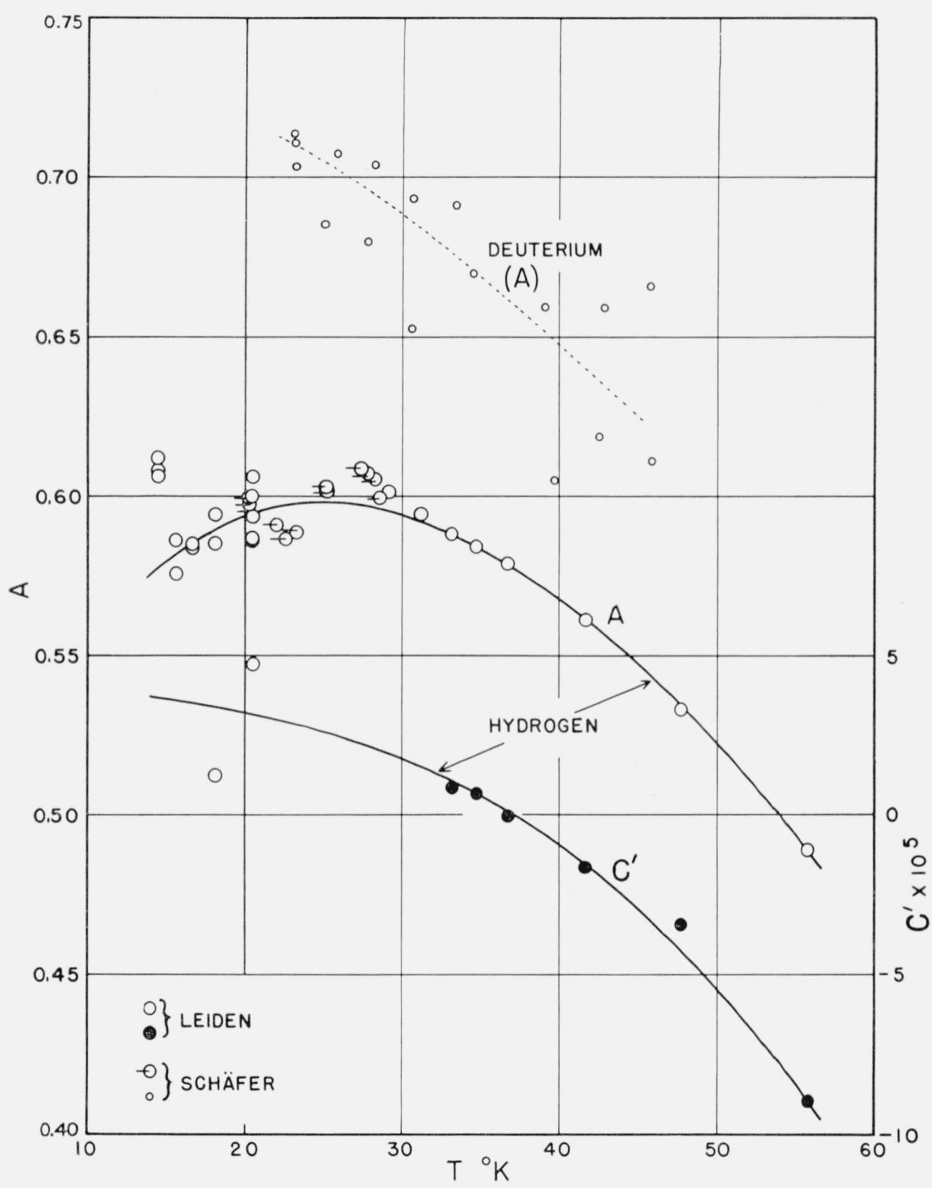

Figure 9. Intercepts and slopes from figure 8.

on the basis of $T_{c}=33.1^{\circ}$ and $P_{c}=12.81 \mathrm{~atm}$, however, unless the critical density is inferred to be about 320, in Amagat units, instead of the reported values 345 or 344 . This difference in critical density seemed too large on the basis of the probable precision of the density measurements The adjustment has instead been so made and the critical isotherm in figure 8 so drawn that $T_{c}=$ $33.19^{\circ}, P_{c}=12.98 \mathrm{~atm}$, and $\rho_{c}=335$. This value of $P_{c}$ is consistent with the PVT data and with therms of figure 8 are straight lines up to $\rho=200$. This assumption was used in correlating the observed data below the critical temperature where the data were scarce and the precision low. In figure 9 the intercepts $A$ and the slopes $C^{\prime}$ of the isotherms of figure 8 are plotted as functions of the temperature. The curve for the slope was extrapolated smoothly to lower temperatures as slopes could not be obtained from the data below $33^{\circ} \mathrm{K}$. 
Also shown in figure 9 are values for $A$ calculated from second virial coefficients determined experimentally by Schäfer [85]. Schäfer reported the results of his PVT measurements as virial coefficients $B^{\prime}(T)=d(P V / R T) / d P$ at constant temperature and at $P=0$. The values of $A=-\left(R T^{5 / 2} /\right.$ $\left.V_{0}\right) B^{\prime}(T)$ obtained from Schäfer's results agree well with those obtained from data of the Leiden Laboratory as shown by figure 9 . Schäfer observed no consistent difference between the second virial coefficients of para hydrogen, normal hydrogen, and a one to one mixture of ortho and para varieties.

The equation for the straight part of the $\psi$ isotherms of figure 8 may be written

$$
\frac{T^{3 / 2}}{\rho}\left(1-\frac{P V}{R T}\right)=A+C \rho
$$

where $C=C^{\prime}-0.0006, C^{\prime}$ being the slopes plotted in figure 9 of the $\psi$-isotherms in figure 8 . Values of $A$ and $C$ and their derivatives are given for hydrogen in table 19 . The values of $P V / R T$ from $\rho=0$ to $\rho=200$ and from $T=14^{\circ}$ to $T=56^{\circ} \mathrm{K}$ in

TABLE 19. Hydrogen values of $A$ and $C$ (and derivatives) in the equation for isotherms

$$
T^{3 / 2} \frac{V}{V_{0}}\left(1-\frac{P V}{R T}\right)=A+C \rho
$$

[Applicable at Amagat densities less than 200]

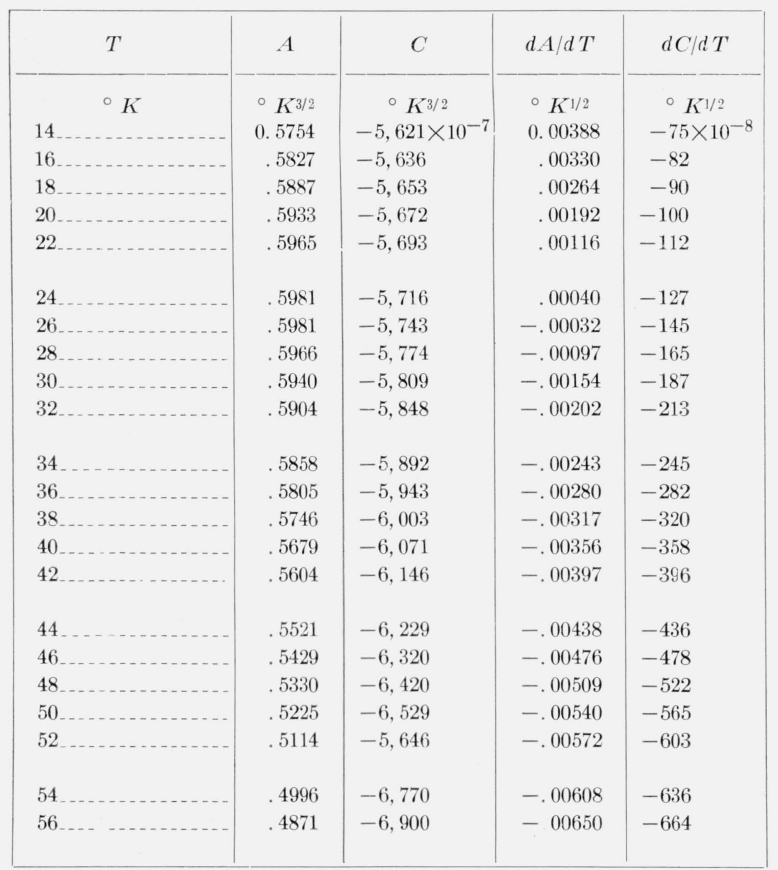

TABLE 20. Pressure, density, and PV/RT for saturated $\mathrm{H}_{2}$ vapor

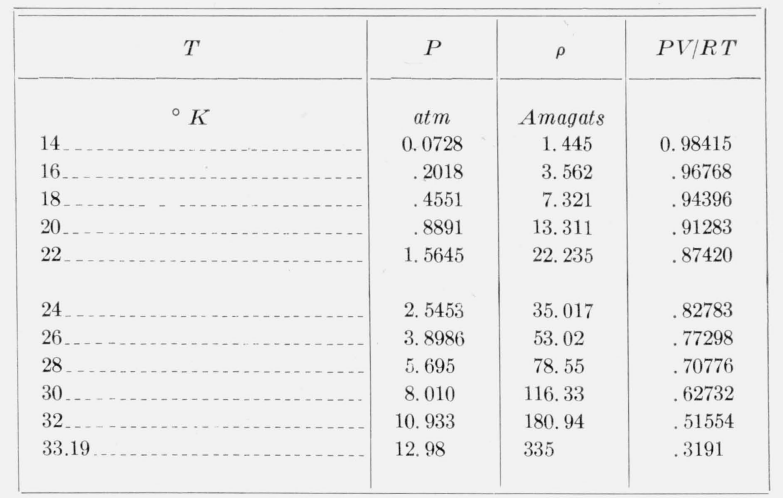

table 13 were calculated using eq 4.14 with table 19. Table 20, giving the pressure, density, and value of $P V / R T$ for saturated $\mathrm{H}_{2}$ vapor, was prepared similarly using the vapor pressure equation for $n-\mathrm{H}_{2}$ (eq 7.2). For certain uses eq 4.14 with table 19 may be more convenient than the tables of $P V / R T$ and its derivatives.

For temperatures below $56^{\circ} \mathrm{K}$ and densities greater than $\rho=200$ where $\psi$ could not be represented by a simple function of $\rho$, a table was made of values of $\psi$ for each $\rho$ and $T$ entry in the $Z$-table. The $\psi$-values of this table were obtained from figure 8 by graphical interpolation. Large plots of $\psi$-isochores, 20 Amagat units apart, on $\psi$ versus $T$ graphs were made of values of $\psi$ read from figure 8 . Values of $\psi$ at 2-degree intervals were read from the isochores. $A Z(\rho, T)$ table was calculated from the $\psi(\rho, T)$ table.

From $56^{\circ}$ to $273^{\circ} \mathrm{K}$, the $\sigma$-function rather than the $\psi$-function was used because above $56^{\circ} \mathrm{K}$ the $\sigma$-isotherms approach linear functions of the density. The method of graphical interpolation used below $56^{\circ} \mathrm{K}$ was used above, also, to obtain a table of $\sigma$-values for the $\rho$ and $T$ entries of the Z-table. The accuracy of graphical interpolation was improved by using more sensitive plots than figure 6 of modified $\sigma$-functions obtained by adding to $\sigma$ simple functions of $T$ and $\rho$, which brought the isotherms and isochores closer together so that they could be easily plotted to a large scale. Values of $\sigma$ were obtained at densities as high as $\rho=500$, although between $70^{\circ}$ and $200^{\circ} \mathrm{K}$ measurements were not available at densities this high. This region was filled in by extrapolation of $\sigma$ curves to higher densities along isotherms and by interpolation along isochores between the upper 
and lower temperature regions where there were data to determine the trend. From the $\sigma(\rho, T)$ table a $Z(\rho, T)$ table was obtained by calculation.

The $Z(\rho, T)$ table obtained through graphical interpolation of the $\psi$ and $\sigma$ isotherms as has just been described was smoothed along isotherms and along isochores by inspection of second differences. In general the $Z$-tables are smooth to one unit in the last digit.

The tables of $(d Z / d T)_{\rho}$ and $(d Z / d \rho)_{T}$ below $0^{\circ} \mathrm{C}$ were for the most part calculated from the smoothed $Z$ table by the method of Rutledge [179] for the calculation of derivatives from smooth sets of tabular values of data. ${ }^{8}$ In the region below $56^{\circ} \mathrm{K}$ and $\rho=200$, where the $\psi$ versus $\rho$ isotherms are straight lines, the following equations, obtained by differentiating eq 4.14, were used with table 19 to calculate the derivatives

$$
\begin{aligned}
& \left(\frac{d Z}{d T}\right)_{\rho}=\frac{3}{2} \frac{(1-Z)}{T}-\frac{\rho}{T^{3 / 2}} \frac{d A}{d T}-\frac{\rho^{2}}{T^{3 / 2}} \frac{d C}{d T} \\
& \left(\frac{d Z}{d \rho}\right)_{T}=-\frac{1}{T^{3 / 2}}\left[A+2\left(C^{\prime}-0.0006\right) \rho\right] .
\end{aligned}
$$

Where the derivatives could be obtained both by the method of Rutledge and by eq 4.15 and 4.16 , the agreement was very satisfactory. The $(d Z / d \rho)_{T}$ and $(d Z / d T)_{\rho}$ tables were also smoothed along isotherms and isochores by inspection of second differences.

The $\left(d^{2} Z / d T^{2}\right)_{\rho}$ table below $0^{\circ} \mathrm{C}$ was obtained throughout by the method of Rutledge from the smoothed $(d Z / d T)_{\rho}$ table and was also smoothed. The equation for $\left(d^{2} Z / d T^{2}\right)_{\rho}$ corresponding to eq 4.15 for the first derivative was considered too involved for easy computation.

In general, the tables of derivatives are smooth to the last digit recorded.

\section{(c) Reliability of Tables of PVT Data}

By inspecting figures 6 to 8 it is possible to arrive at some general conclusions regaräing the deviations of the observed data from the $Z(\rho, T)$ table. It may be noted that, except at low densities, the deviations of the observational values of $\sigma$ from the curves representing the table are of about the same magnitude at different densities along a given isotherm up to $\rho=500 .^{9}$ This means that deviations of $(P V / R T)-1$

\footnotetext{
${ }^{8}$ Assuming that differences of higher order than the fourth are negligible.
}

${ }^{9}$ For still greater densities larger deviations occur as shown by figure 7 . along an isotherm are approximately proportional to the density. At low densities the deviations are large because the sensitivity of the $\sigma$ and $\psi$ plots approaches infinity as $\rho$ approaches zero. It is difficult to make an estimate of the probable error in $P V / R T$ based on the deviations because, as is seen, the greatest deviations are the systematic differences between the results of different observers and are not accidental errors as should be the case if error theory were to apply. The user of the tables can make an estimate of the mean difference between the observed and tabulated values of $P V / R T$, in any particular region of temperature and density by noting the deviations shown on the graph and from these calculating the corresponding deviations in $P V / R T$. For temperatures below $60^{\circ} K$ it would be best to use figure 8 for this purpose as it is plotted to a larger scale than is figure 6 .

In constructing the tables for the intermediate temperature regions where analytical equations of state were not used, just enough digits were retained so that changes made in smoothing would be confined to the last digit. As a considerable amount of smoothing resulted from the graphical methods used, many of the irregularities in the measured values were not apparent in the unsmoothed tables.

It is believed that throughout the table the values were carried out to at least as many significant figures as were at all justified by the data, and that the last digit recorded should be considered very uncertain. In that part of the table between $77^{\circ}$ and $200^{\circ} \mathrm{K}$ which was filled in by interpolation and extrapolation the last two digits should be considered uncertain, the last recorded digit being retained to achieve continuity with the rest of the table.

The tables are thought to be most reliable for temperatures between $273^{\circ}$ and $373^{\circ} \mathrm{K}\left(0^{\circ}\right.$ and $\left.100^{\circ} \mathrm{C}\right)$, because at these temperatures the experimental difficulties encountered are not as great as at higher and lower temperatures. Also, as is shown by figure 6 the results of several different investigators are in agreement at these temperatures. Above $373^{\circ} \mathrm{K}$ the experimental data are not as self-consistent as at temperatures immediately below. As the values of $P V / R T$ given in the tables for these higher temperatures are derived largely from an extrapolation based on the temperature region between $273^{\circ}$ and $373^{\circ} \mathrm{K}$, 
an estimate of reliability of the high temperature portion of the tables involves both the applicability of the correlating function, eq 4.8, and the precision of the experimental data. Considering the differences between the isothermal lines determined by different sets of experimental data of different observers and the same observer at different temperatures, it seems probable that the extrapolation is more reliable than the experimental data at temperatures above $473^{\circ} \mathrm{K}$.

It is doubted that $P V / R T$ is known to better than 0.2 percent for densities as high as 100 Amagats near $33^{\circ} \mathrm{K}$, the critical temperature.

Below the critical temperature, the data are not very satisfactory. In addition to the difficulties of making measurements at low temperatures, there exists the circumstance that below the critical temperature the range of vapor densities that can be covered is limited by the density of saturated vapor. At low densities the deviations $(1-Z)$ from the ideal gas law are small and hence difficult to measure precisely.

There is another method of obtaining values of second virial coefficients which may be advantageous for the low temperature region. It involves the determination of the velocity of sound, which has been carried out for gaseous hydrogen at liquid-hydrogen temperatures and various pressures by van Itterbeek and Keesom [77], using a resonance method. The change of the velocity of sound with pressure at very low pressures is related to the value of the second virial coefficient and to its first and second derivatives. Because of this relationship, it is possible to determine the second virial coefficient from the velocity of sound if the second virial coefficient is already known in an adjacent range of temperature. Van Itterbeek and Keesom concluded that the agreement between their own measurements and the PVT data was "rather good", although for both types of data the scattering was quite appreciable.

In calculating the tables of derivatives by the method of Rutledge, the criterion for retaining significant figures in the recorded values was the same as that previously mentioned, namely, enough places were carried so that the changes resulting from the smoothing were in general confined to the last digit. As in the case of the tables of $P V / R T$, it is believed that the tabulated values of the derivatives are given to as many significant figures as are justified by the data.

\section{Deuterium}

The interesting features of the PVT data for deuterium are most evident when deuterium is compared with hydrogen. The difference between the second virial coefficients of $\mathrm{H}_{2}$ and $\mathrm{D}_{2}$ has been investigated theoretically $[86,87]$, though a complete treatment of the problem has not been made.

Assuming the same intermolecular forces for $\mathrm{H}_{2}$ and $\mathrm{D}_{2}$, classical mechanics and statistics lead to the same equation of state for $\mathrm{H}_{2}$ and $\mathrm{D}_{2}$. The quantum theory of virial coefficients leads to effective volumes of molecules and to second virial coefficients that are larger than the classical values, the differences being small at ordinary temperatures but becoming large at low temperatures. ${ }^{10}$

In table 21 are given ratios between quantum mechanical and classical values of second virial coefficients, for gases whose molecules are rigid nonattracting spheres. They may also be considered as ratios between apparent molecular volumes for the two treatments. These ratios are based on formulas derived by Uhlenbeck and Beth [84]. Columns 2 and 3 are for gases with molecular weights 2 and 4 , respectively. The value of the ratio depends, among other things, upon the diameters of the rigid spheres. Here the size of the spheres was taken to be the same for the two

TABLE 21. Ratio between quantum mechanical and classical second virial coefficients for nonattracting rigid spherical molecules a of molecular weight $M$

\begin{tabular}{|c|c|c|}
\hline$T$ & $\frac{B \text { quantum }}{B \text { classical }}$ for $M=2$ & $\frac{B \text { quantum }}{B \text { classical }}$ for $M=4$ \\
\hline${ }^{\circ} K$ & & \\
\hline $600 \ldots$ & 1. 21 & 1.15 \\
\hline 300 & 1. 30 & 1. 21 \\
\hline $100 \ldots$ & 1. 52 & 1.37 \\
\hline $25 \ldots \ldots \ldots$ & 2.7 & 2.0 \\
\hline $5 \ldots$ & 4. 6 & 2. 6 \\
\hline
\end{tabular}

a With diameters calculated from the van der Waals' $b$ for hydrogen.

10 The application of quantum mechanies instead of ordinary mechanics has as one effect for rigid spherical molecules the removal of the classical discontinuity in the calculated distribution of molecules for pair separations corresponding to contact between the spheres. As smaller separations are prevented by the impenetrability of the spheres, the continuity is established by a reduction of the molecular density for separations greater than that corresponding to contact. The effect is large for separations of sphere surfaces up to a considerable fraction of the de Broglie wavelength (for which $h / \sqrt{2 m k T}$ is a representative value) and depends through this upon the temperature. This reduction of molecular density beyond the minimum separation could be represented roughly in a classical description as an increase of the volume from which 1 molecule causes the centers of other molecules to be excluded. In classical theory the second virial coefficient for nonattracting rigid spheres is proportional to the excluded volume. 
gases and to be equal to the size calculated from the van der Waals $b$ for $\mathrm{H}_{2}$.

Although it would scarcely be expected that the results of calculations for rigid nonattracting spheres would apply to real $\mathrm{H}_{2}$ and $\mathrm{D}_{2}$ molecules, it would seem likely that qualitative indications would be correct, at least at higher temperatures where the excluded volume predominates over the intermolecular attractive forces in determining the magnitude of the second virial coefficient. This is borne out by experiment, the difference in second virial coefficients $\left(B_{\mathrm{H}_{2}}-B_{\mathrm{D}_{2}}\right)$, being positive, though smaller than would be indicated by table 21 for rigid spheres by a factor of about 2.6 at $300^{\circ} \mathrm{K}$. Uhlenbeck and Beth derived an approximate quantum mechanical representation for the second virial coefficient applicable at high

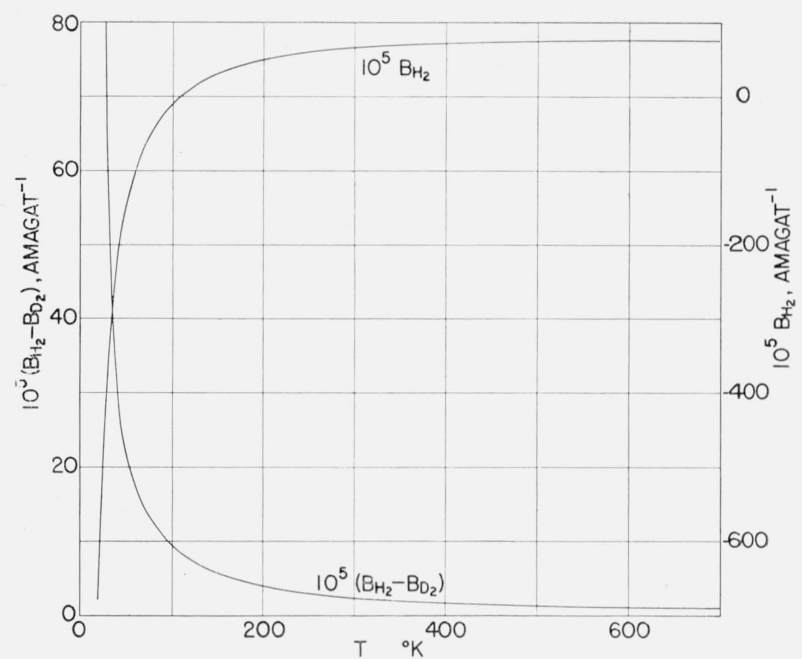

Figure 10. Second virial coefficient for $\mathrm{H}_{2}$ and the difference between second virial coefficients for $\mathrm{H}_{2}$ and $\mathrm{D}_{2}$.

temperatures for molecules with radially symmetrical force fields. Their formulas were applied to hydrogen and deuterium by de Boer and Michels [87] upon the assumption that the intermolecular forces were the same for $\mathrm{H}_{2}$ and $\mathrm{D}_{2}$. They obtained differences between the virial coefficients for $\mathrm{H}_{2}$ and $\mathrm{D}_{2}$ represented by the upper temperature portion of one of the curves of figure 10. In a later paper by Michels and Goudeket [92] attention was called to the fact that the intermolecular forces of hydrogen and deuterium do differ a little because the mean internuclear separations of $\mathrm{H}_{2}$ and $\mathrm{D}_{2}$ molecules are different as a result of the different zero point vibrations of their nuclei.
The effect of the intermolecular attractive forces overbalances the effect of the excluded volume or the repulsive forces of the molecules in determining the magnitude of the second virial coefficient at low temperatures, and makes the coefficient negative. Nevertheless, at low temperatures, as at high temperatures, the difference in second virial coefficients $B_{\mathrm{H}_{2}}-B_{\mathrm{D}_{2}}$ is positive, partly for the reason already discussed in the case of high temperatures, namely the larger apparent quantum-mechanical volume of $\mathrm{H}_{2}$ molecules, and partly for another reason. There is a closer spacing of the discrete negative energy states and smaller zero point energy for pairs of $\mathrm{D}_{2}$ molecules than for pairs of $\mathrm{H}_{2}$ molecules because of the mass difference, so that by reason of the Boltzmann factor, $\exp [-$ energy/kT], there is a greater degree of association or clustering together of $\mathrm{D}_{2}$ molecules than of $\mathrm{H}_{2}$ molecules. Without a consideration of the Boltzmann factors for these negative energy levels the effect of the difference of mass would be less clear, as the quantum treatment for the continuum would require that the spacing of the levels there be smaller for $\mathrm{D}_{2}$ than for $\mathrm{H}_{2}$ in essentially the same ratio as in the case of the discrete negative energy levels. With these or similar ideas in mind, Schäfer [86] derived a formula for the difference in second virial coefficients for $\mathrm{H}_{2}$ and $\mathrm{D}_{2}$ at low temperatures, which involved a constant whose magnitude he so chose as to obtain a fit with his experimental values for the difference in the second virial coefficients.

Figure 9 shows values of $A$ in the equation of state (eq 4.14) calculated from the second virial coefficients of deuterium for the temperature range $23^{\circ}$ to $45^{\circ}$ determined experimentally by Schäfer [85].

$$
A=-T^{3 / 2}(d Z / d \rho)_{T, \rho=0}=-T^{3 / 2} B_{1},
$$

where $B_{1}$ is the second virial coefficient in the equation of state $P V=R T\left(1+B_{1} \rho+B_{2} \rho^{2}+\ldots\right)$. The dashed line curve in figure 9 was obtained by adding to the $A$ 's for $\mathrm{H}_{2}$ the differences between the $A$ 's calculated from the differences between the second virial coefficients of $\mathrm{H}_{2}$ and $\mathrm{D}_{2}$ which Schäfer determined partly theoretically and partly empirically. Schäfer's measurements were made on deuterium at low densities and hence do not give information on higher virial coefficients. Approximate values of $P V$ for deuterium at low 
temperatures may be found by using values of $A$ from figure 9 in eq 4.14, and either neglecting the $C$ term or preferably using the corresponding value of $C$ for $\mathrm{H}_{2}$.

Values of the function $\sigma=\left(T V / T_{0} V_{0}\right) \quad \log _{10}$ $(P V / R T)$ calculated from the data of Michels and Goudeket [92] for $\mathrm{D}_{2}$ are shown as open circles in figure 7 . The dashed straight lines for deuterium are obtainable from the equation

$$
P V / R T=\exp \left[B(T) \rho+C(T) \rho^{2}\right],
$$

where

$$
\begin{aligned}
B(T)= & 0.0055298 T^{-1 / 4}-0.036040 T^{-3 / 4}- \\
& 0.25878 T^{-5 / 4}
\end{aligned}
$$

and

$$
C(T)=0.00580 T^{-3 / 2}-0.0565 T^{-2} .
$$

The constants in the formula for $B$ have been so chosen that the difference between $\mathrm{D}_{2}$ and $\mathrm{H}_{2}$ intercepts on the $\sigma$-axis is in close agreement with the theoretical result of de Boer and Michels [87] from $250^{\circ}$ to $450^{\circ} \mathrm{K}$.

In figure 10 , a curve marked $10^{5}\left(B_{H_{2}}-B_{D_{2}}\right)$ shows the trend of differences between second virial coefficients based on the theoretical calculations above $150^{\circ} \mathrm{K}$ and on the results of Schäfer below $50^{\circ} \mathrm{K}$ with an interpolation between. It may be inferred that the differences between the PV'T data for $\mathrm{H}_{2}$ and $\mathrm{D}_{2}$ decrease rather rapidly with increase of temperature. For comparison, the curve marked $10^{5} B_{H_{2}}$, in figure 10 , shows on a different scale the magnitude of the corresponding second virial coefficient for $\mathrm{H}_{2}$ at the same temperatures.

If it is assumed that the $\sigma$ or $\left(T V / T_{0} V_{0}\right) \log$ $(P V / R T)$ isotherms for $\mathrm{D}_{2}$ and $\mathrm{H}_{2}$ are parallel, values of $P V / R T$ for $\mathrm{D}_{2}$ may be obtained from those tabulated for $\mathrm{H}_{2}$ by (1) calculating the $\sigma_{\mathrm{H}_{2}}$ or $\sigma$ for $\mathrm{H}_{2}$, from the values of $P V / R T, T$ and $\rho$, (2) subtracting the difference $\left(\sigma_{H_{2}}-\sigma_{D_{2}}\right)_{\rho=0}$ to get $\sigma_{D_{2}}$, and then (3) calculating the corresponding value of $P V / R T$ for $\mathrm{D}_{2}$. A plot of the difference $10^{5}\left(\sigma_{H_{2}}-\sigma_{D_{2}}\right)_{\rho=0}$ which may be used for this purpose is shown in figure 11. An alternative method based on the assumption that only the second term of the series expansion eq 4.9 for $P V / R T$ is to be changed is as follows. $10^{5}$
$\left(B_{H_{2}}-B_{D_{2}}\right)$, obtained from figure 11 by multiplying $10^{5}\left(\sigma_{H_{2}}-\sigma_{D_{2}}\right)_{\rho=0}$ by $2.302585 T_{0} / T$ or obtained directly from figure 10 , is multiplied by $10^{-5}$ and the product subtracted from $P V / R T$ for $\mathrm{H}_{2}$ to give $P V / R T$ for $\mathrm{D}_{2}$. This alternative method is simpler than the other method and may be as reliable.

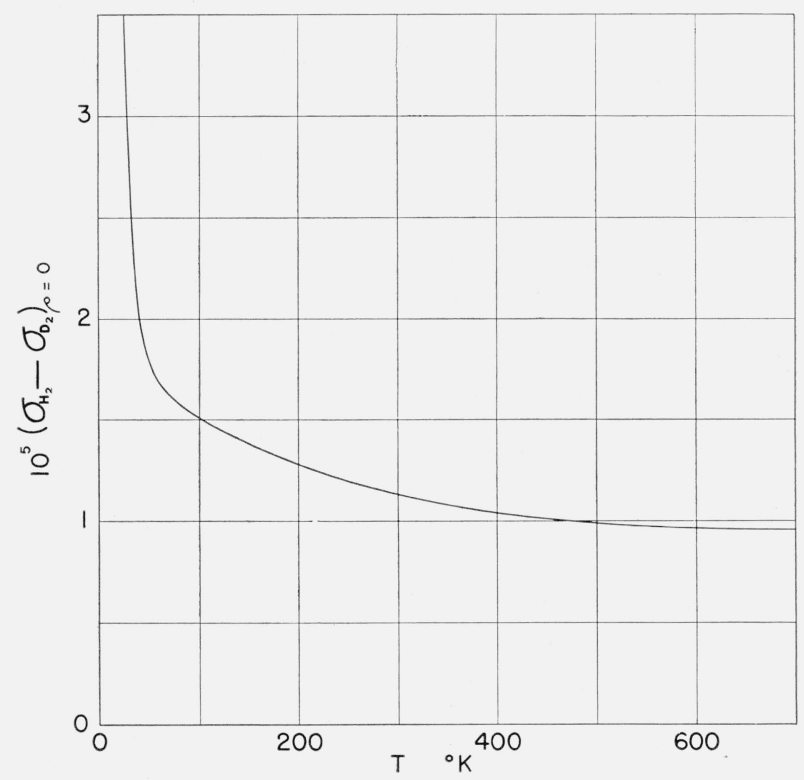

Figure 11. Difference between intercepts of $\sigma$ versus $\rho$ isotherms for $\mathrm{H}_{2}$ and $\mathrm{D}_{2}$.

\section{Calculation of Thermal Properties of the Real Gas}

The calculation of thermodynamic properties of a real gas from values of these properties for the ideal gas rests upon the principle that the difference between values of a thermodynamic function at different densities for the same temperature may be determined from data of state for the gas at the given temperature.

The entropy and free energy of a gas are dependent upon the pressure, even in the ideal state, and in tables 4 to 8 they are given for the hydrogens in the ideal gas state at a pressure of 1 standard atm. On the other hand, the internal energy, enthalpy, and specific heat in the ideal gas state are independent of density at constant temperature.

Equations 5.1 to 5.8 show how, using the data of state expressed in the form, $Z=Z(\rho, T)$, the thermodynamic properties of the real gas at a temperature $T$ and an Amagat density $\rho$ may be calculated from properties for the ideal gas state 
at a pressure of $1 \mathrm{~atm}$, given for the hydrogens in tables 4 to 8 .

$$
\begin{aligned}
\frac{S_{\rho, T} \text { (real gas) }}{R}= & \frac{S_{P=1, T \text { (ideal) }}^{\circ}+\ln \frac{P_{0} V_{0}}{R T_{0}}-\ln \frac{T}{T_{0}}-\ln \rho-}{R} \\
& \int_{0}^{\rho}[(Z-1) / \rho] d \rho-\int_{0}^{\rho}\left[T(d Z / d T)_{\rho} / \rho\right] d \rho
\end{aligned}
$$

This can be expressed in a slightly different form by using the identity

$$
\begin{gathered}
\int_{0}^{\rho}[(Z-1) / \rho] d \rho+\int_{0}^{\rho}\left[T(d Z / d T)_{\rho} / \rho\right] d \rho= \\
\left(d\left\{T \int_{0}^{\rho}[(Z-1) / \rho] d \rho\right\} / d T\right) .
\end{gathered}
$$

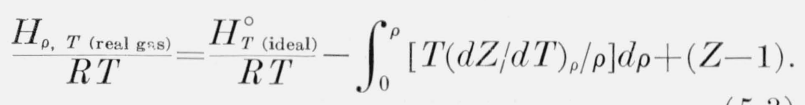

$$
\begin{aligned}
\frac{F_{\rho, T \text { (real gas })}^{R T}=}{R T} & \frac{F_{P=1, T \text { (ideal) }}^{\circ}-\ln \frac{P_{0} V_{0}}{R T_{0}}+\ln \frac{T}{T_{0}}+\ln \rho+}{R T} \\
& \int_{0}^{\rho}[(Z-1) / \rho] d \rho+(Z-1) .
\end{aligned}
$$

$\ln [f$ (fugacity of real gas) $\left./ P]=\int_{0}^{\rho}(Z-1) / \rho\right] d \rho-$

$$
\ln Z+(Z-1) \text {. }
$$

$$
\begin{gathered}
\frac{E_{\rho, T \text { (real gas) }}}{R T}=\frac{E_{T}^{\circ \text { (ideal) }}}{R T}-\left[T(d Z / d T)_{\rho} / \rho\right] d \rho . \\
\frac{\left(C_{v}\right)_{\rho, T \text { (real gas) }}}{R}=\frac{\left(C_{v}^{\circ}\right)_{T} \text { (ideal) }}{R}-2 \int_{0}^{\rho}\left[T(d Z / d T)_{\rho} / \rho\right] d \rho \\
-\int_{0}^{\rho}\left[T^{2}\left(d^{2} Z / d T^{2}\right)_{\rho} / \rho\right] d \rho . \\
\frac{\left(C_{P}\right)_{\rho, T \text { (real gas) }}=}{R}=\frac{\left(C_{P}^{\circ}\right)_{T} \text { (ideal) }}{R}- \\
2 \int_{0}^{\rho}\left[T(d Z / d T)_{\rho} / \rho\right] d \rho-\int_{0}^{\rho}\left[T^{2}\left(d^{2} Z / d T^{2}\right)_{\rho} / \rho\right] d \rho+ \\
\left\{\left[Z+T(d Z / d T)_{\rho}\right]^{2} /\left[Z+\rho(d Z / d \rho)_{T}\right]\right\}-1 .
\end{gathered}
$$

In order to facilitate the calculation of the thermodynamic properties of hydrogen in the real gas state, tables 22 and 23 were computed. ${ }^{11}$ Lagrangian four point formulas [181] were used for the tabular integrations.

Table 22 is intended for use in the calculation of

\footnotetext{
11 For the calculation of these tables the authors are indebted to Messrs Roger E. Clapp, Kingsley Elder, Jr., and Robert Mann, who worked as student assistants at the National Bureau of Standards during the summer of 1941 .
}

entropies. The values in the second column, headed $\left(S_{p=1}^{\circ}-S_{p=1}^{\circ}\right) / R$, are for the difference between entropies of hydrogen in the ideal gas state at 1-atm pressure and at unit Amagat density, divided by $R$.

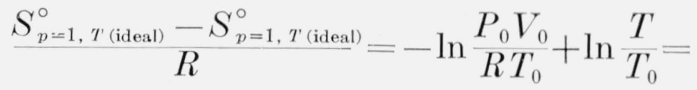

$$
\begin{aligned}
& -0.000618+\ln T / T_{0}
\end{aligned}
$$

The row at the bottom of the table, headed $\left(S_{\rho=1}^{\circ}-S^{\circ}\right) / R$, is for the difference between entropies in the ideal gas states at Amagat densities one and $\rho$, divided by $R$.

$$
\frac{S_{p=1, T \text { (ideal) }}^{\circ}-S_{p, T \text { (ideal) }}^{\circ}}{R}=\ln \rho
$$

The other rows and columns of table 22 headed $\left(S^{\circ}-S\right) / R$ give the differences between the entropies in the ideal and real gas states at the same temperature and density, divided by $R$.

$$
\begin{aligned}
& \frac{S_{\rho, T \text { (ideal) }}^{\circ}-S_{\rho, T \text { (real) }}}{R}=\int_{0}^{\rho}[(Z-1) / \rho] d \rho+ \\
& \int_{0}^{\rho}\left[T(d Z / d T)_{\rho} / \rho\right] d \rho
\end{aligned}
$$

In order, then, to get $S / R$ for the real gas hydrogen at a temperature $T$ and Amagat density $\rho$, one subtracts from $S^{\circ} / R$, obtained from $S^{\circ}$ given in table 8 , the sum of three numbers for the appropriate values of $T$ and $\rho$ to be obtained from table 22: one comes from the second column, headed $\left(S_{p=1}^{\circ}-S_{\rho=1}^{\circ}\right) / R$; another from the bottom row of the table, headed $\left(S_{\rho=1}^{\circ}-S^{\circ}\right) / R$; and the third from the rows and columns of the table headed $\left(S^{\circ}-S\right) / R$.

Table 23 is for the difference between the enthalpy of hydrogen in the ideal and real gas states at temperature $T$ and Amagat density $\rho$, divided by $R T$.

$\frac{H_{T \text { (ideal }}^{\circ}-H_{\rho, T \text { (real) }}}{R T}=\int_{0}^{\rho}\left[T(d Z / d T)_{\rho} / \rho\right] d \rho-(Z-1)$.

Hence to obtain $H / R T$ for hydrogen in the real gas state, one subtracts the appropriate value of $\left(H^{\circ}-H_{\rho}\right) / R T$ in table 23 from the value of $H^{\circ} / R T$ obtained from $H^{\circ}$ given in table 8 for the deal gas state. 
TABLE 22. Entropy differences divided by $R$, for normal $\mathrm{H}_{2}$

$\frac{S^{\circ}-S}{R}$, Entropy of ideal gas minus entropy of real gas at same $T$ and $\rho$, divided by $R$.

$\frac{S_{P(=1)}^{\circ}-S_{(\rho=11}^{\circ}}{R}$, Entropy of ideal gas at pressure of 1 atmosphere minus entropy of ideal gas at density of 1 Amagat, divided by $P$.

$\frac{\left.S_{P}^{\circ}=1\right)-S^{\circ}}{R}$, Entropy of ideal gas at density of 1 A magat minus entropy of ideal gas at density of $\rho$ A magats, divided by $R$.

\begin{tabular}{|c|c|c|c|c|c|c|c|c|c|c|c|c|c|c|c|c|}
\hline \multirow{2}{*}{$\begin{array}{l}\text { Temper- } \\
\text { ature }\end{array}$} & \multirow{2}{*}{$\frac{S_{(P=1)}^{0}-S_{(\rho=1)}^{\circ}}{R}$} & \multicolumn{15}{|c|}{$\frac{S^{\circ}-S}{R}$} \\
\hline & & $\begin{array}{c}\rho=1 \\
\text { Amagat }\end{array}$ & 2 & 3 & 6 & 10 & 20 & 40 & 60 & 80 & 100 & 120 & 140 & 160 & 180 & 200 \\
\hline${ }_{16}^{\circ} \mathrm{K}$ & -2.83809 & 0.00373 & 0.00745 & 0.01117 & & & & & & & & & & & & \\
\hline $18 \ldots \ldots$ & -2.72030 & .00323 & .00645 & .00968 & 0.01933 & & & & & & & & & & & \\
\hline $20 \ldots \ldots$ & -2.61494 & .00288 & .00576 & .00864 & .01724 & 0.0287 & & & & & & & & & & \\
\hline $22 \ldots$ & -2.51963 & .00264 & .00529 & .00792 & .01583 & .0263 & & & & & & & & & & \\
\hline $24 \ldots \ldots$ & -2.43262 & .00245 & .00489 & .00733 & .01463 & .0245 & 0.0488 & & & & & & & & & \\
\hline $26 \ldots \ldots$ & -2.35258 & .00230 & .00459 & .00690 & .01378 & .0230 & .0458 & 0.0915 & & & & & & & & \\
\hline $28 \ldots \ldots$ & -2.27847 & .00218 & .00435 & .00653 & .01304 & .0218 & .0436 & .0867 & 0.129 & & & & & & & \\
\hline $30 \ldots$ & -2.20948 & .00208 & .00415 & .00623 & .01245 & .0208 & .0416 & .0827 & .123 & 0.163 & 0. 202 & & & & & \\
\hline $32 \ldots \ldots$ & -2.14494 & .00198 & .00396 & .00595 & .01189 & .0198 & .0396 & .0787 & .117 & .155 & .193 & 0.230 & 0.266 & 0.302 & 0.337 & \\
\hline $34 \ldots .$. & -2.08432 & .00190 & .00379 & .00569 & .01137 & .0190 & .0379 & .0753 & .112 & .148 & .184 & .220 & .255 & .290 & .324 & 0.358 \\
\hline $36 \ldots$ & -2.02716 & .00182 & .00363 & .00545 & .01088 & .0181 & .0362 & .0719 & .107 & .142 & .176 & .211 & .245 & .278 & .311 & .344 \\
\hline $38 \ldots .$. & -1.97309 & .00175 & .00349 & .00524 & .01047 & .0174 & .0347 & .0692 & .103 & .137 & .170 & .203 & .236 & .268 & .300 & .332 \\
\hline $40 \ldots$ & -1.92180 & .00169 & .00338 & .00508 & .01014 & .0169 & .0337 & .0672 & .100 & .133 & .165 & .198 & .229 & .261 & .293 & .324 \\
\hline $42 \ldots$ & -1.87301 & .00165 & .00329 & .00494 & .00988 & .0165 & .0329 & .0655 & .098 & .130 & .161 & .193 & .225 & .256 & .287 & .317 \\
\hline $44 \ldots$ & -1.82649 & .00160 & .00320 & .00480 & .00960 & .0160 & .0320 & .0640 & .096 & .128 & .158 & .190 & .221 & .252 & .283 & .313 \\
\hline $46 \ldots$ & -1.78204 & .00156 & .00313 & .00469 & .00938 & .0156 & .0313 & .0626 & .094 & .125 & .156 & .186 & .217 & .248 & .278 & .308 \\
\hline $48 \ldots$ & -1.73948 & .00153 & .00306 & .00460 & .00919 & .0153 & .0306 & .0613 & .092 & .122 & .153 & .183 & .213 & .244 & .274 & .303 \\
\hline $50 \ldots$ & -1.69865 & .00150 & .00300 & .00451 & .00902 & .0150 & .0301 & .0601 & .090 & .120 & .150 & .180 & .210 & .240 & .270 & .299 \\
\hline $52 \ldots$ & -1.65943 & .00148 & .00295 & .00443 & .00886 & .0148 & .0295 & .0591 & .089 & .118 & .148 & .177 & .207 & .237 & .266 & .295 \\
\hline $54 \ldots$ & -1.62169 & .00146 & .00291 & .00436 & .00873 & .0146 & .0291 & .0582 & .088 & .117 & .146 & .175 & .204 & .234 & .262 & 291 \\
\hline $56 \ldots$ & -1.58532 & .00144 & .00287 & .00431 & .00862 & .0144 & .0287 & .0575 & .087 & .116 & .144 & .173 & .202 & .231 & .259 & .288 \\
\hline $58 \ldots$ & -1.55023 & .00142 & .00284 & .00426 & .00851 & .0142 & .0284 & .0568 & .086 & .114 & .142 & .171 & .200 & .228 & .257 & .285 \\
\hline $60 \ldots$ & -1.51633 & .00140 & .00280 & .00420 & .00840 & .0140 & .0280 & .0560 & .084 & .112 & .140 & .168 & .197 & .225 & .254 & .283 \\
\hline $65 \ldots$ & -1.43629 & .00135 & .00270 & .00405 & .00810 & .01352 & .0271 & .0540 & .0812 & .109 & .136 & .164 & .192 & .219 & .248 & .276 \\
\hline $70 \ldots$ & -1.36218 & .00131 & .00261 & .00392 & .00784 & .01309 & .0263 & .0525 & .0790 & .106 & .132 & .159 & .186 & .213 & .241 & .269 \\
\hline $75 \ldots$ & -1.29319 & .00127 & .00254 & .00381 & .00762 & .01271 & .0254 & .0510 & .0768 & .103 & .129 & .155 & .182 & .209 & .236 & .263 \\
\hline $80 \ldots$ & -1.22865 & .00124 & .00248 & .00371 & .00743 & .01240 & .0248 & .0499 & .0752 & .101 & .126 & .152 & .179 & .205 & .232 & .259 \\
\hline $85 \ldots .$. & -1.16802 & .00121 & .00242 & .00364 & .00728 & .01214 & .0244 & .0490 & .0739 & .099 & .124 & .150 & .176 & .202 & .228 & .254 \\
\hline $90 \ldots$ & -1.11087 & .00119 & .00238 & .00357 & .00714 & .01192 & .0239 & .0481 & .0726 & .097 & .122 & .148 & .173 & .199 & .225 & .251 \\
\hline $95 \ldots \ldots$ & -1.05680 & .00117 & .00234 & .00351 & .00702 & .01171 & .0234 & .0472 & .0712 & .096 & .120 & .145 & .170 & .196 & .221 & .247 \\
\hline $100 \ldots$ & $-1.00 \Sigma 51$ & .00115 & .00229 & .00344 & .00689 & .01149 & .0230 & .0464 & .0700 & .094 & .118 & .143 & .168 & .193 & .218 & .244 \\
\hline $105 \ldots$ & -0.95672 & .00113 & .00226 & .00339 & .00679 & .01133 & .0227 & .0458 & .0691 & .093 & .117 & .141 & .165 & .190 & .215 & .241 \\
\hline $110 \ldots$ & -.91020 & .00112 & .00224 & .00335 & .00671 & .01120 & .0224 & .0452 & .0683 & .092 & .115 & .139 & .163 & .188 & .213 & .238 \\
\hline $115 \ldots$ & -.86574 & .00111 & .00222 & .00333 & .00665 & .01111 & .0222 & .0448 & .0677 & .091 & .114 & .138 & .162 & .186 & .211 & .236 \\
\hline $120 \ldots$ & -.82318 & .00110 & .00220 & .00331 & .00661 & .01104 & .0221 & .0445 & .0672 & .090 & .113 & .137 & .161 & 185 & .209 & .234 \\
\hline
\end{tabular}




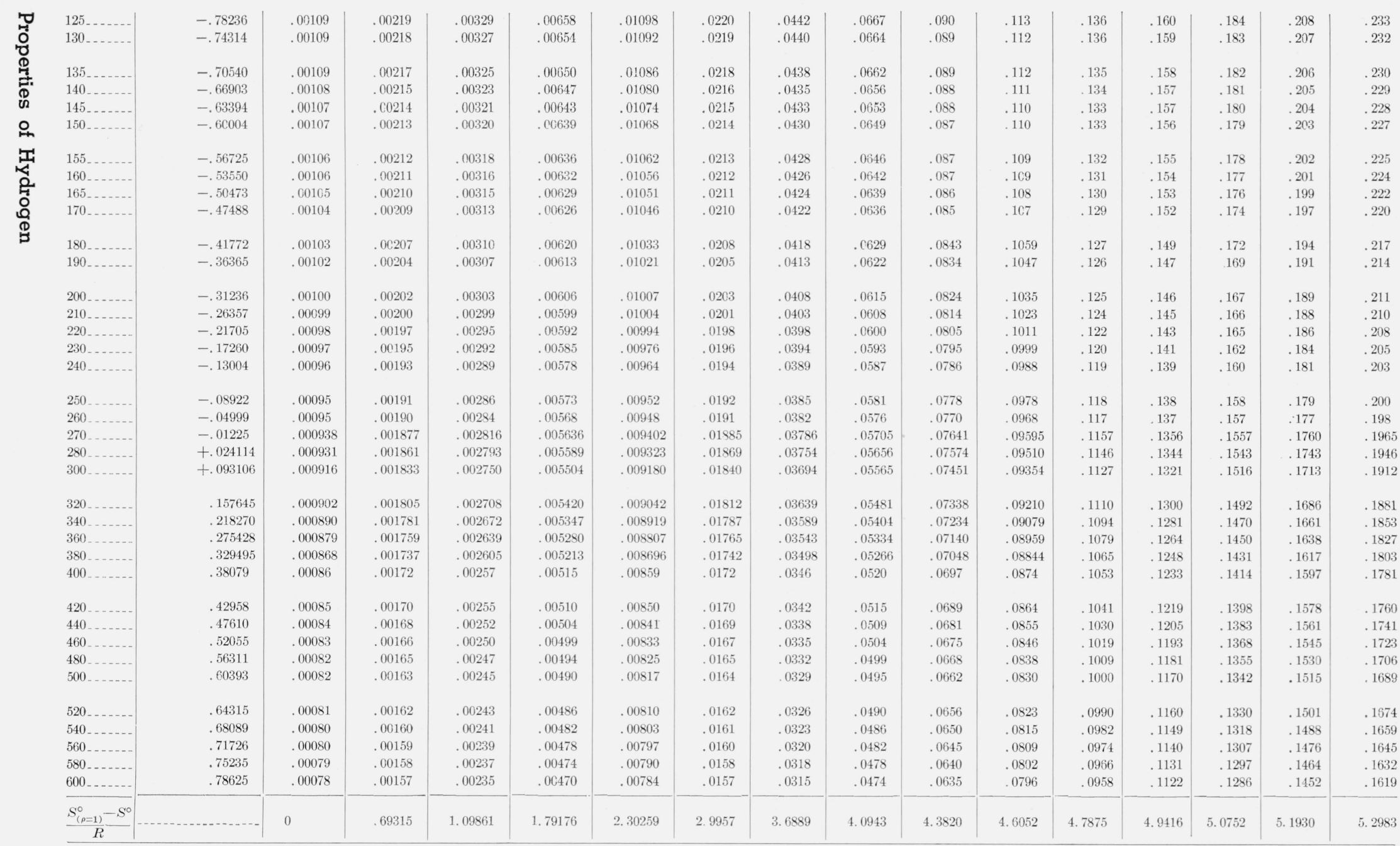


Table 22. Entropy differences divided by $R$, for normal $\mathrm{H}_{2}$-Continued

\begin{tabular}{|c|c|c|c|c|c|c|c|c|c|c|c|c|c|c|c|c|}
\hline \multirow{2}{*}{$\begin{array}{l}\text { Temper- } \\
\text { ature }\end{array}$} & \multirow{2}{*}{$\frac{S_{(P=1)}^{\circ}-S_{(p=1)}^{\circ}}{R}$} & \multicolumn{15}{|c|}{$\frac{S^{\circ}-S}{R}$} \\
\hline & & $\rho=220$ & 240 & 260 & 280 & 300 & 320 & 340 & 360 & 389 & 490 & 420 & 440 & 460 & 180 & 500 \\
\hline \\
\hline \multirow{2}{*}{\multicolumn{17}{|c|}{$\begin{array}{l}18 \\
20\end{array}$}} \\
\hline & & & & & & & & & & & & & & & & \\
\hline \multicolumn{17}{|l|}{$\begin{array}{l}22 . \\
24\end{array}$} \\
\hline \multicolumn{17}{|l|}{$\begin{array}{l}24 \ldots \\
26 \ldots\end{array}$} \\
\hline \multirow{2}{*}{\multicolumn{17}{|c|}{$28 \ldots$}} \\
\hline $30 \ldots$ & & & & & & & & & & & & & & & & \\
\hline $32 \ldots$ & & & & & & & & & & & & & & & & \\
\hline $34 \ldots$. & -2.0843 & 0.391 & 0.424 & 0.457 & 0.489 & 0.520 & 0.551 & 0.581 & 0.610 & 0.638 & 0.666 & 0.694 & 0.723 & 0.753 & 0.784 & 0.816 \\
\hline $36 \ldots$ & -2.0272 & .376 & .408 & .441 & .473 & .504 & .535 & .565 & .594 & .623 & .652 & .682 & .712 & .742 & .774 & .807 \\
\hline $38 \ldots \ldots$ & -1.9731 & .364 & .395 & .427 & .458 & .489 & .519 & .550 & .580 & .610 & .640 & .671 & .701 & .733 & .766 & .800 \\
\hline $40 \ldots .$. & -1.9218 & .355 & .386 & .417 & .448 & .478 & .509 & .539 & .570 & .601 & .632 & .664 & .696 & .728 & .762 & .796 \\
\hline $42 \ldots \ldots$ & -1.8730 & .348 & .379 & .409 & .439 & .470 & .500 & .531 & .563 & .594 & .626 & .658 & .691 & .724 & .758 & .792 \\
\hline $44 \ldots$ & -1.8265 & .343 & .374 & .404 & .434 & .465 & .495 & .526 & .557 & .589 & .622 & .655 & .688 & .721 & .755 & .789 \\
\hline $46 \ldots \ldots$ & -1.7820 & .338 & .368 & .399 & .428 & .459 & .489 & .520 & .551 & .583 & .616 & .649 & .682 & .716 & .750 & .784 \\
\hline $48 \ldots \ldots$ & -1.7395 & .333 & .363 & .393 & .422 & .453 & .483 & .514 & .545 & .577 & .609 & .642 & .676 & .710 & .743 & .777 \\
\hline $50 \ldots$ & -1.6987 & .328 & .358 & .388 & .417 & .447 & .478 & .508 & .539 & .571 & .603 & .636 & .669 & .702 & .736 & .769 \\
\hline $52 \ldots \ldots$ & -1.6594 & .325 & .354 & .383 & .413 & .443 & .472 & .503 & .534 & .565 & .596 & .629 & .661 & .694 & .728 & .762 \\
\hline $54 \ldots$ & -1.6217 & .320 & .350 & .379 & .408 & .438 & .467 & .497 & .528 & .559 & .590 & .622 & .654 & .687 & .720 & .754 \\
\hline $56 \ldots \ldots$ & -1.5853 & .317 & .346 & .375 & .405 & .434 & .464 & .494 & .524 & .554 & .586 & .617 & .649 & .682 & .715 & .749 \\
\hline $58 \ldots \ldots$ & -1.5502 & .314 & .343 & .373 & .402 & .432 & .462 & .492 & .523 & .554 & .585 & .616 & .648 & .681 & .715 & .749 \\
\hline $60 \ldots$ & -1.5163 & .312 & .341 & .371 & .401 & .431 & .461 & .492 & .523 & .554 & .586 & .618 & .650 & .683 & .717 & .751 \\
\hline $65_{\ldots} \ldots$ & -1.4363 & .305 & .334 & .363 & .393 & .424 & .454 & .485 & .516 & .548 & .580 & .613 & .646 & .679 & .714 & .749 \\
\hline $70 \ldots$ & -1.3622 & .298 & .326 & .356 & .385 & .415 & .445 & .476 & .507 & .539 & .571 & .604 & .637 & .670 & .705 & .739 \\
\hline $75 \ldots \ldots$ & -1.2932 & .291 & .320 & .348 & .377 & .407 & .436 & .467 & .497 & .529 & .560 & .593 & .625 & .659 & .692 & .727 \\
\hline $80 \ldots$ & -1.2286 & .286 & .314 & .342 & .370 & .399 & .428 & .458 & .488 & .519 & .550 & .582 & .614 & .647 & .680 & .714 \\
\hline $85 \ldots .$. & -1.1680 & .281 & .308 & .336 & .364 & .392 & .421 & .450 & .480 & .510 & .540 & .571 & .603 & .635 & .667 & .700 \\
\hline $90 \ldots$ & -1.1109 & .278 & .304 & .332 & .359 & .387 & .416 & .445 & .474 & .503 & .534 & .564 & .595 & .627 & .659 & .691 \\
\hline $95 \ldots \ldots$ & -1.0568 & .274 & .301 & .328 & .355 & .383 & .411 & .440 & .469 & .498 & .528 & .558 & .589 & .620 & .652 & .684 \\
\hline $100 \ldots$ & -1.0055 & .270 & .297 & .324 & .351 & .379 & .407 & .435 & .464 & .493 & .522 & .552 & .583 & .614 & .645 & .677 \\
\hline $105 \ldots \ldots$ & --0.9567 & .267 & .294 & .320 & .347 & .375 & .402 & .430 & .459 & .487 & .517 & .546 & .576 & .607 & .638 & .670 \\
\hline $110 \ldots \ldots$ & -.9102 & .264 & .290 & .317 & .344 & .371 & .398 & .426 & .454 & .482 & .511 & .540 & .570 & .600 & .631 & .662 \\
\hline 115 & -.8657 & .262 & .287 & .313 & .340 & .367 & .394 & .421 & .449 & .477 & .505 & .534 & .564 & .594 & .624 & .655 \\
\hline 120 & -.8232 & .259 & .285 & .311 & .337 & .364 & .390 & .418 & .445 & .473 & .501 & .530 & .559 & .589 & .619 & .649 \\
\hline 125 & -.7824 & .258 & .284 & .309 & .335 & .361 & .388 & .415 & .442 & .470 & .498 & .526 & .555 & .584 & .614 & .644 \\
\hline 130 & -.7431 & .257 & .282 & .307 & .333 & .359 & .386 & .412 & .439 & .467 & .495 & .523 & .552 & .580 & .610 & .640 \\
\hline $135 \ldots$ & -.7054 & .255 & .280 & .305 & .331 & .357 & .383 & .409 & .436 & .463 & .491 & .519 & .547 & .576 & .605 & .634 \\
\hline $140 \ldots$ & -.6690 & .253 & .278 & .303 & .329 & .354 & .380 & .407 & .433 & .460 & .488 & .515 & .544 & .572 & .601 & .630 \\
\hline $145 \ldots \ldots$ & -.6339 & .252 & .277 & .301 & .327 & .352 & .378 & .404 & .431 & .457 & .485 & .512 & .540 & .568 & .597 & .626 \\
\hline $150 \ldots \ldots$ & -.6000 & .251 & .275 & .300 & .325 & .351 & .376 & .402 & .429 & .455 & .482 & .509 & .537 & .565 & .593 & .622 \\
\hline
\end{tabular}




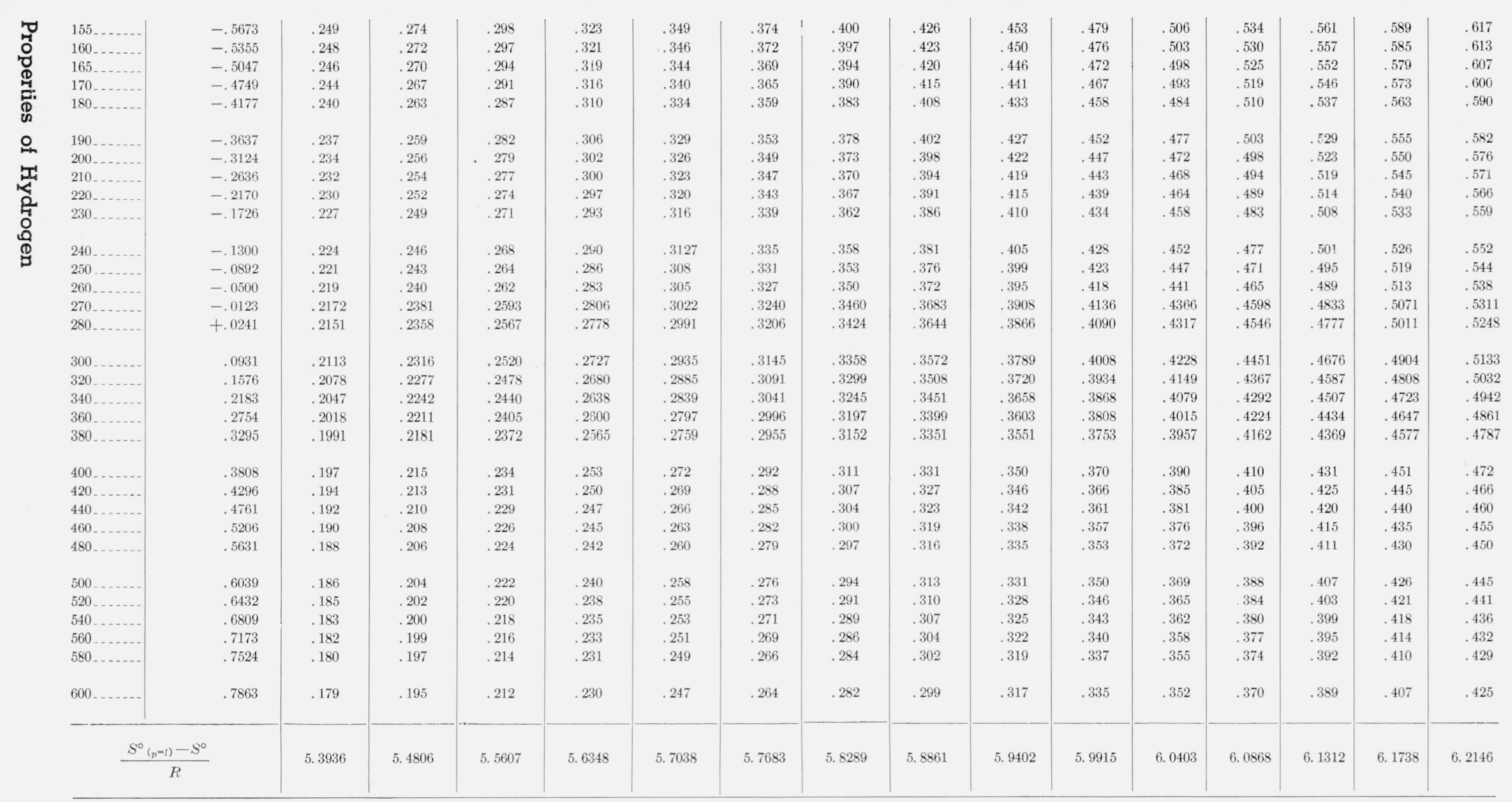


TABLE 23. Enthalpy of ideal gas minus enthalpy of real gas at the same $T$ and $\rho$, divided by $R T$, for normal $H_{2}$

\begin{tabular}{|c|c|c|c|c|c|c|c|c|c|c|c|c|c|c|c|}
\hline \multirow{2}{*}{ Temperature } & \multicolumn{15}{|c|}{$\frac{H^{\circ}-H}{R T}$} \\
\hline & $\rho=1 \mathrm{~A}$ magat & 2 & 3 & 6 & 10 & 20 & 40 & 60 & 80 & 100 & 120 & 140 & 160 & 180 & 200 \\
\hline${ }^{\circ} \mathrm{K}$ & 0. 02193 & 0.04381 & 0.06568 & & & & & & & & & & & & \\
\hline 18 & .01869 & .03725 & .05583 & 0.11144 & & & & & & & & & & & \\
\hline $20 \ldots$ & .01614 & .03226 & .04835 & .09650 & 0.1604 & & & & & & & & & & \\
\hline $22 \ldots$ & .01420 & .02838 & .04253 & .08489 & .1411 & & & & & & & & & & \\
\hline $24 \ldots$ & .01263 & .02524 & .03783 & .07551 & .1255 & 0.2494 & & & & & & & & & \\
\hline $26 \ldots$ & .01132 & .02260 & .03388 & .06765 & .1125 & .2237 & 0.4420 & & & & & & & & \\
\hline $28 \ldots$ & .01023 & .02045 & .03063 & .06116 & .1017 & .2023 & .3994 & 0.591 & & & & & & & \\
\hline $30 \ldots$ & .00931 & .01860 & .02786 & .05564 & .0925 & .1839 & .3637 & .538 & 0.708 & 0.872 & & & & & \\
\hline $32 \ldots$ & .00851 & .01701 & .02549 & .05089 & .0846 & .1681 & .3318 & .491 & .646 & .797 & 0.943 & 1. 085 & 1. 222 & 1. 355 & \\
\hline $34 \ldots$ & .00781 & .01556 & .02337 & .046666 & .0776 & .1541 & .3042 & .451 & .593 & .731 & .865 & 0.995 & 1. 121 & 1. 243 & 1. 361 \\
\hline $36 \ldots$ & .00719 & .01436 & .02153 & .04298 & .0714 & .1420 & .2801 & .414 & .545 & .673 & .796 & .916 & 1. 032 & 1. 145 & 1. 253 \\
\hline $38 \ldots$ & .00665 & .01229 & .01991 & .03976 & .0661 & .1313 & .2592 & .383 & .504 & .622 & .736 & .847 & 0.955 & 1. 059 & 1. 160 \\
\hline $40 \ldots$ & .00617 & .01234 & .01850 & .03692 & .0614 & .1220 & .2409 & .357 & .469 & .578 & .684 & .787 & .887 & 0.984 & 1. 078 \\
\hline $42 \ldots$ & .00576 & .01151 & .01727 & .03445 & .0573 & .1140 & .2249 & .333 & .438 & .539 & .638 & .734 & .828 & .918 & 1. 005 \\
\hline $44 \ldots$ & .00538 & .01077 & .01615 & .03223 & .0536 & .1067 & .2104 & .312 & .410 & .505 & .598 & .688 & .775 & .869 & 0.942 \\
\hline $46 \ldots$ & .00504 & .01008 & .01513 & .03020 & .0502 & .1000 & .1971 & .292 & .384 & .473 & .560 & .645 & .727 & .806 & .883 \\
\hline $48 \ldots$ & .00473 & .00946 & .01420 & .02834 & .0471 & .0937 & .1849 & .274 & .360 & .444 & .526 & .605 & .682 & .757 & .829 \\
\hline $50 \ldots$ & 00445 & .00889 & .01324 & .02665 & .0443 & .0881 & .1739 & .257 & .338 & .417 & .494 & .569 & .641 & .711 & .779 \\
\hline $52 \ldots$ & .00419 & .00837 & .01257 & .02509 & .0417 & .0830 & .1638 & .213 & .319 & .394 & .466 & .537 & .605 & .671 & .734 \\
\hline $54 \ldots$ & .00396 & .00792 & .01188 & .02371 & .0393 & .0784 & .1548 & .229 & .302 & .373 & .441 & .507 & .571 & .632 & .692 \\
\hline $56 \ldots$ & .00376 & .00750 & .01126 & .02247 & .0374 & .0743 & .1466 & .217 & .286 & .353 & .417 & .479 & .540 & .598 & .654 \\
\hline $58 \ldots$ & .00356 & .00710 & .01066 & .02127 & .0354 & .0704 & .1389 & .206 & .271 & .333 & .394 & .453 & .511 & .566 & .619 \\
\hline $60 \ldots$ & .00337 & .00674 & .01010 & .02017 & .0335 & .0666 & .1315 & .194 & .256 & .315 & .373 & .429 & .483 & .536 & .586 \\
\hline $65 \ldots$ & .00296 & .00592 & .00887 & .01771 & .02943 & .0584 & .1153 & .1706 & .2242 & .276 & .327 & .376 & .423 & .469 & .513 \\
\hline $70 \ldots$ & .00261 & .00522 & .00782 & .01542 & .02595 & .0515 & .1017 & .1503 & .1974 & .243 & .287 & .330 & .371 & .411 & .449 \\
\hline $75 \ldots$ & .00231 & .09461 & .00692 & .01382 & .02297 & .0456 & .0900 & .1330 & .1747 & .215 & .254 & .291 & .327 & .362 & .395 \\
\hline $80 \ldots$ & .00205 & .00411 & .00616 & .01230 & .02043 & .0406 & .0799 & .1182 & .1552 & .191 & .225 & .258 & .290 & .320 & .349 \\
\hline $85 \ldots$ & .00184 & .00368 & .00551 & .01101 & .01828 & .0363 & .0714 & .1055 & .1384 & .170 & .201 & .230 & .257 & .283 & .308 \\
\hline $90 \ldots . .$. & .00165 & .00330 & .00495 & .00987 & .01639 & .0325 & .0639 & .0942 & .1235 & .152 & .179 & .204 & .229 & .252 & .273 \\
\hline $95 \ldots$ & .00148 & .00296 & .00444 & .00884 & .01469 & .0291 & .0571 & .0842 & .1101 & .135 & .159 & .181 & .203 & .223 & .241 \\
\hline $100 \ldots$ & .00133 & .00265 & .00398 & .00793 & .01316 & .0261 & .0511 & .0752 & .0983 & .120 & .141 & .161 & .179 & .197 & .213 \\
\hline 105 & .00119 & .00238 & .00357 & .00712 & .01182 & .0234 & .0458 & .0673 & .0878 & .107 & .125 & .143 & .159 & .174 & .187 \\
\hline $110 \ldots$ & .00107 & .00215 & .00322 & .00642 & .01065 & .0210 & .0412 & .0603 & .0785 & .095 & .111 & .126 & .140 & .153 & .165 \\
\hline 115 & .00097 & .00194 & .00290 & .00579 & .00960 & 0190 & .0370 & .0540 & .0701 & .085 & .099 & .112 & .124 & .135 & .144 \\
\hline 120 & .00088 & .00175 & .00262 & .00523 & .00867 & .0171 & .0333 & .0485 & .0628 & .076 & .088 & .099 & .109 & .118 & .126 \\
\hline $125 \ldots$ & .00079 & .00158 & .00236 & .00472 & .00781 & .0154 & .0299 & .0434 & .0561 & .068 & .078 & .088 & .097 & .104 & .110 \\
\hline $130 \ldots$ & .00071 & .00143 & .00213 & .00425 & .00704 & .0139 & .0268 & .0388 & .0499 & .060 & .069 & .077 & .084 & .090 & .095 \\
\hline 135 & .00064 & .00128 & .00193 & .00382 & .00632 & .0124 & .0239 & .0345 & .0442 & .053 & .061 & .067 & .073 & .078 & .081 \\
\hline $140 \ldots$ & .00057 & .00115 & .00172 & .00341 & .00563 & .0110 & .0212 & .0305 & .0389 & .046 & .053 & .058 & .063 & .066 & .068 \\
\hline $145 \ldots$ & .00051 & .00102 & .00154 & .00304 & .00502 & .0098 & .0186 & .0269 & .0340 & .040 & .045 & .050 & .053 & .055 & .056 \\
\hline $150 \ldots$ & .00045 & .00090 & .00136 & .00269 & .00444 & .0087 & .0165 & .0235 & .0295 & .035 & .039 & .042 & .044 & .045 & .015 \\
\hline
\end{tabular}




\begin{tabular}{|c|c|c|c|c|c|c|c|c|c|c|c|c|c|c|c|}
\hline $155_{-.}$ & .00040 & .00081 & .00121 & .00239 & .00393 & .0076 & .0144 & .0204 & .0254 & .029 & .033 & .035 & .036 & .036 & .035 \\
\hline $160 \ldots$ & .00035 & .00071 & .00106 & .00209 & .00344 & .0067 & .0125 & .0174 & .0214 & .024 & .027 & .028 & .028 & .027 & .025 \\
\hline $165 \ldots$ & .00031 & .00061 & .00092 & .00180 & .00295 & .0057 & .0105 & .0145 & .0176 & .020 & .021 & .021 & .020 & .018 & .015 \\
\hline $170_{\ldots}$ & .00026 & .00052 & .00076 & .00152 & .00248 & .0047 & .0087 & .0117 & .0139 & .015 & .015 & .014 & .012 & .010 & .006 \\
\hline 180 & .00017 & .00034 & .00050 & .00100 & .00161 & .0030 & .0052 & .0066 & .0070 & .0065 & .005 & .003 & -.001 & -.005 & -.011 \\
\hline $190 \ldots$ & .00010 & .00019 & .00028 & .00053 & .00085 & .0015 & .0022 & .0021 & .0011 & -.0008 & -.004 & -.098 & -.012 & -.018 & -.025 \\
\hline $200 \ldots$ & .00003 & .00006 & .00008 & .00015 & .00021 & .0002 & -.0003 & -.0017 & -.0059 & -.0070 & -.011 & -.016 & -.022 & -.029 & -.037 \\
\hline $210 \ldots$ & -.00003 & -.00005 & -.00009 & -.00020 & -.00037 & -.0009 & -.0026 & -.0051 & -.0084 & -.0126 & -.018 & -.024 & -.031 & -.039 & $\begin{array}{l}-.008 \\
-.048\end{array}$ \\
\hline $220 \ldots$ & -.00008 & -.00016 & -.00024 & -.00051 & -.00091 & -.0020 & -.0047 & -.0082 & -.0126 & -.0178 & -.024 & -.031 & -.039 & -.047 & -.057 \\
\hline $230 \ldots$ & -.00013 & -.00027 & -.00041 & -.00084 & -.00143 & -.0030 & -.0068 & -.0113 & -.0166 & -.0228 & -.030 & -.038 & -.047 & -.056 & -.067 \\
\hline $240 \ldots$ & -.00018 & -.00036 & -.00056 & -.00113 & -.00192 & -.0040 & -.0088 & -.0142 & -.0204 & -.0274 & -.035 & -.044 & -.054 & -.064 & -.076 \\
\hline $250 \ldots$ & -.00023 & -.00046 & -.00068 & -.00138 & -.00233 & -.0048 & -.0104 & -.0166 & -.0236 & -.0314 & -.040 & -.050 & -.060 & -.071 & -.083 \\
\hline 260 & -.00026 & -.00053 & -.00078 & -.00159 & -.00268 & -.0055 & -.0118 & -.0187 & -.0265 & -.0349 & -.044 & -.054 & -.065 & -.077 & -.090 \\
\hline $270 \ldots$ & -.000290 & -.000584 & -.000877 & -.001770 & -.002985 & -.00615 & -.01299 & -.02058 & -.02891 & -.03802 & -.0479 & -.0587 & -.0703 & -.0828 & -.0962 \\
\hline $280 \ldots$ & -.000321 & -.000645 & -.000969 & -.001953 & -.003290 & -.00675 & -.01421 & -.02238 & -.03131 & -.04099 & -.0515 & -.0628 & -.0749 & -.0879 & -.1018 \\
\hline $300 \ldots$ & -.000375 & -.000752 & -.001131 & -.002276 & -.003827 & -.00782 & -.01634 & -.02556 & -.03549 & -.04620 & -.0577 & -.0699 & -.0830 & -.0970 & -.1118 \\
\hline 320 & -.000422 & -.000845 & -.001271 & -.002556 & -.004291 & -.00375 & -.01816 & -.02826 & -.03907 & -.05062 & -.0629 & -.0760 & -.0899 & -.1046 & -.1202 \\
\hline $340_{-}$ & -.006462 & -.000926 & -.001350 & -.002794 & -.064688 & -.00954 & -.01973 & -.03058 & -.04213 & -.05440 & -.0674 & -.0812 & -.6957 & -.1111 & -.1273 \\
\hline $360_{\ldots}$ & -.000496 & -.000994 & -.001493 & -.003000 & -.005031 & -.01022 & -.02108 & -.03259 & -.04478 & -.05766 & -.0713 & -.0856 & -.1007 & -.1166 & -.1334 \\
\hline $380 \ldots$ & -.000526 & -.001054 & -.001584 & -.003181 & -.005332 & -.01081 & -.02226 & -.03433 & -.04707 & -.06049 & -.6746 & -.0895 & -.1051 & -.1214 & -.1386 \\
\hline $400 \ldots$ & -.00055 & -.00111 & -.00166 & -.00334 & -.00560 & -.0113 & -.0233 & -.0359 & -.0491 & -.0630 & -.0776 & -.0928 & -.1088 & -.1256 & -.1432 \\
\hline $420 \ldots$ & -.00058 & -.00116 & -.00174 & -.00348 & -.00583 & -.0118 & -.0242 & -.0372 & -.0509 & -.0652 & -.0801 & -.0958 & -.1121 & -.1292 & -.1471 \\
\hline $440 \ldots$ & -.00060 & -.06120 & -.00180 & -.00360 & -.00604 & -.0122 & -.0250 & -.0384 & -.0524 & -.0671 & -.0824 & -.0984 & -.1150 & -.1324 & -.1506 \\
\hline $460 \ldots$ & -.00062 & -.00123 & -.00185 & -.00372 & -.00622 & -.0126 & -.0257 & -.0395 & -.0538 & -.0688 & -.0844 & -.1006 & -.1176 & -.1352 & -.1536 \\
\hline $480_{\ldots} \ldots$ & -.00063 & -.00127 & -.00190 & -.00382 & -.00639 & -.0129 & -.0264 & -.0404 & -.0550 & -.0703 & -.0862 & -.1027 & -.1199 & -.1377 & -.1563 \\
\hline $500 \ldots$ & -.00065 & -.00130 & -.00195 & -.00390 & -.00653 & -.0132 & -.0270 & -.0413 & -.0562 & -.0716 & -.0877 & -.1045 & -.1218 & -.1399 & -.1587 \\
\hline $520 \ldots$ & -.00066 & -.00132 & -.00199 & -.00398 & $-.0 v 667$ & -.0135 & -.0275 & -.0420 & -.0571 & -.0728 & -.0892 & -.1061 & -.1236 & -.1419 & -.1608 \\
\hline $540_{\ldots} \ldots$ & -.00067 & -.00135 & -.00202 & -.00406 & -.00679 & -.0137 & -.0279 & -.0427 & -.0580 & -.0739 & -.0904 & -.1075 & -.1252 & -.1436 & -.1627 \\
\hline $560 \ldots$ & -.00068 & -.00137 & -.00205 & -.00412 & -.00689 & -.0139 & -.0284 & -.0433 & -.0588 & -.0749 & -.0916 & -.1088 & -.1267 & -.1452 & -.1643 \\
\hline $580_{\ldots} \ldots$ & -.00069 & -.00139 & -00208 & -.00418 & -.00699 & -.0141 & -.0287 & -.0439 & -.0596 & -.0758 & -.0926 & -.1100 & -.1280 & -.1466 & -.1658 \\
\hline $600 \ldots$ & -.00070 & -.00141 & -.00211 & -.00424 & -.00708 & -.0143 & -.0291 & -.0444 & -.0602 & -.0766 & -.0935 & -.1110 & -.1291 & -.1478 & -.1671 \\
\hline
\end{tabular}




\begin{tabular}{|c|c|c|c|c|c|c|c|c|c|c|c|c|c|c|c|}
\hline \multirow{2}{*}{ Temperature } & \multicolumn{15}{|c|}{$\frac{H^{\circ}-H}{R T}$} \\
\hline & $\rho=220$ & 240 & 260 & 280 & 300 & 320 & 340 & 360 & 380 & 400 & 420 & 440 & 460 & 480 & 500 \\
\hline \multicolumn{16}{|l|}{${ }^{\circ} \mathrm{K}$} \\
\hline \multicolumn{16}{|l|}{$18 \ldots$} \\
\hline $20 \ldots$ & & & & & & & & & & & & & & & \\
\hline \multicolumn{16}{|l|}{22} \\
\hline \multicolumn{16}{|l|}{$\begin{array}{l}24 . \\
26 .\end{array}$} \\
\hline \multirow{2}{*}{\multicolumn{16}{|c|}{$28 \ldots$}} \\
\hline \multicolumn{9}{|l|}{$30 \ldots$} & & & & & & & \\
\hline 32 & & & & & & & & & & & & & & & \\
\hline $34 \ldots$ & 1. 475 & 1. 582 & 1. 692 & 1. 793 & 1. 890 & 1. 983 & 2. 071 & 2. 154 & 2. 234 & 2. 310 & 2. 382 & 2. 453 & 2. 521 & 2. 586 & 2. 647 \\
\hline $36 \ldots$ & 1. 359 & 1. 461 & 1. 559 & 1. 653 & 1. 743 & 1. 830 & 1.912 & 1. 991 & 2. 065 & 2. 136 & 2. 204 & 2. 269 & 2. 331 & 2. 390 & 2. 446 \\
\hline $38 \ldots \ldots$ & 1. 257 & 1. 351 & 1. 442 & 1. 529 & 1. 612 & 1. 693 & 1. 770 & 1. 844 & 1. 914 & 1. 981 & 2. 044 & 2. 104 & 2. 161 & 2. 215 & 2. 265 \\
\hline $40 \ldots$ & 1. 168 & 1. 256 & 1. 340 & 1. 421 & 1. 499 & 1. 574 & 1. 646 & 1.715 & 1. 781 & 1. 843 & 1. 902 & 1. 958 & 2. 010 & 2. 059 & 2. 104 \\
\hline $42 \ldots$ & 1. 090 & 1. 171 & 1. 250 & 1.325 & 1. 398 & 1.467 & 1. 534 & 1. 599 & 1. 661 & 1. 719 & i. 774 & 1.826 & 1. 874 & 1. 919 & 1.959 \\
\hline $44 \ldots$ & 1. 021 & 1. 097 & 1. 170 & 1. 241 & 1. 308 & 1.373 & 1. 436 & 1. 496 & 1. 553 & 1. 608 & 1. 659 & 1. 707 & 1. 752 & 1. 792 & 1. 829 \\
\hline $46 \ldots$ & 0.957 & 1. 028 & 1. 097 & 1. 163 & 1. 226 & 1. 286 & 1. 344 & 1. 400 & 1. 453 & 1. 504 & 1. 551 & 1. 596 & 1. 637 & 1. 674 & 1. 707 \\
\hline $48 \ldots$ & .898 & 0.965 & 1. 029 & 1. 091 & 1. 150 & 1. 206 & 1. 260 & 1.311 & 1. 361 & 1. 407 & 1. 451 & 1. 492 & 1. 530 & 1. 564 & 1. 594 \\
\hline $50 \ldots$ & .844 & .907 & 0.968 & 1. 025 & 1. 081 & 1. 133 & 1. 183 & 1. 231 & 1. 276 & 1. 319 & 1. 359 & 1. 396 & 1. 430 & 1. 461 & 1. 489 \\
\hline $52 \ldots$ & .796 & .855 & .911 & 0.966 & 1. 017 & 1. 066 & 1.112 & 1.156 & 1. 198 & 1. 237 & 1. 273 & 1. 307 & 1. 338 & 1. 366 & 1. 391 \\
\hline $54 \ldots$ & .750 & .806 & .859 & .910 & 0.958 & 1. 003 & 1. 046 & 1. 087 & 1. 125 & 1. 161 & 1. 195 & 1. 225 & 1. 253 & 1. 279 & 1. 301 \\
\hline $56 \ldots$ & .708 & .761 & .811 & .859 & .904 & 0.947 & 0.987 & 1. 025 & 1. 060 & 1. 093 & 1. 123 & 1. 151 & 1. 177 & 1. 200 & 1. 219 \\
\hline $58 \ldots$ & .670 & .720 & .767 & .812 & .855 & .896 & .934 & 0.969 & 1. 002 & 1. 033 & 1. 061 & 1.087 & 1. 110 & 1.130 & 1.148 \\
\hline $60 \ldots$ & .635 & .682 & .727 & .770 & .811 & .849 & .886 & .919 & 0.950 & 0.979 & 1. 005 & 1. 028 & 1. 049 & 1. 069 & 1.085 \\
\hline $65 \ldots$ & .555 & .596 & .634 & .671 & .706 & .739 & .770 & .799 & .825 & .849 & 0.870 & 0.889 & 0.906 & 0.920 & 0.931 \\
\hline $70_{\ldots} \ldots$ & .486 & .521 & .554 & .586 & .615 & .643 & .669 & .692 & .714 & .733 & .750 & .764 & .776 & .786 & .793 \\
\hline $75 \ldots$ & .427 & .457 & .485 & .511 & .536 & .559 & .580 & .599 & .616 & .631 & .644 & .654 & .662 & .667 & .669 \\
\hline $80 \ldots$ & .376 & .401 & .425 & .447 & .468 & .487 & .503 & .518 & .531 & .542 & .551 & .557 & .561 & .562 & .561 \\
\hline $85 \ldots$ & .331 & .353 & .373 & .391 & .408 & .423 & .436 & .448 & .457 & .464 & .469 & .472 & .472 & .470 & .466 \\
\hline $90 \ldots$ & .293 & .312 & .328 & .344 & .357 & .369 & .380 & .388 & .394 & .398 & .400 & .400 & .398 & .393 & .385 \\
\hline $95 \ldots$ & .259 & .274 & .289 & .301 & .312 & .322 & .329 & .335 & .338 & .340 & .340 & .337 & .332 & .325 & .315 \\
\hline $100 \ldots$ & .228 & .241 & .253 & .263 & .272 & .279 & .284 & .287 & .288 & .288 & .285 & .280 & .274 & .264 & .252 \\
\hline $105 \ldots$ & .200 & .211 & .221 & .229 & .235 & .240 & .242 & .244 & .243 & .240 & .236 & .229 & .220 & .208 & . 195 \\
\hline $110 \ldots$ & .175 & .184 & .191 & .197 & .202 & .204 & .205 & .204 & .202 & .197 & .191 & .182 & .171 & .158 & .142 \\
\hline $115_{\ldots} \ldots$ & .152 & .159 & .165 & .169 & .171 & .172 & .172 & .169 & 164 & .158 & .150 & .140 & .127 & .112 & .095 \\
\hline $120 \ldots$ & .132 & .137 & .141 & .144 & .144 & .144 & .141 & .137 & .131 & .123 & .114 & .102 & .088 & .072 & .053 \\
\hline $125 \ldots$ & .115 & .118 & .121 & .121 & .121 & .118 & .115 & .109 & .102 & .093 & .082 & .068 & .053 & .036 & .016 \\
\hline $130 \ldots$ & .098 & .100 & .101 & .101 & .099 & .095 & .090 & .083 & .075 & .064 & .052 & .038 & .021 & .003 & -.018 \\
\hline $135 \ldots$ & .083 & .084 & .083 & .081 & .078 & .073 & .067 & .059 & .049 & .038 & .024 & .009 & -.008 & -.028 & -.050 \\
\hline 140 & .068 & .068 & .066 & .063 & .059 & .053 & .046 & .037 & .026 & .014 & -.001 & -.017 & -.036 & -.056 & -.079 \\
\hline $145 \ldots$ & .056 & .054 & .051 & .047 & .041 & .035 & .026 & .016 & .005 & -.009 & -.024 & -.042 & -.061 & -.082 & -.106 \\
\hline 150 & .044 & .041 & .037 & .032 & .026 & .018 & .009 & -.002 & -.015 & -.029 & -.045 & -.064 & -.084 & -.106 & -.130 \\
\hline
\end{tabular}




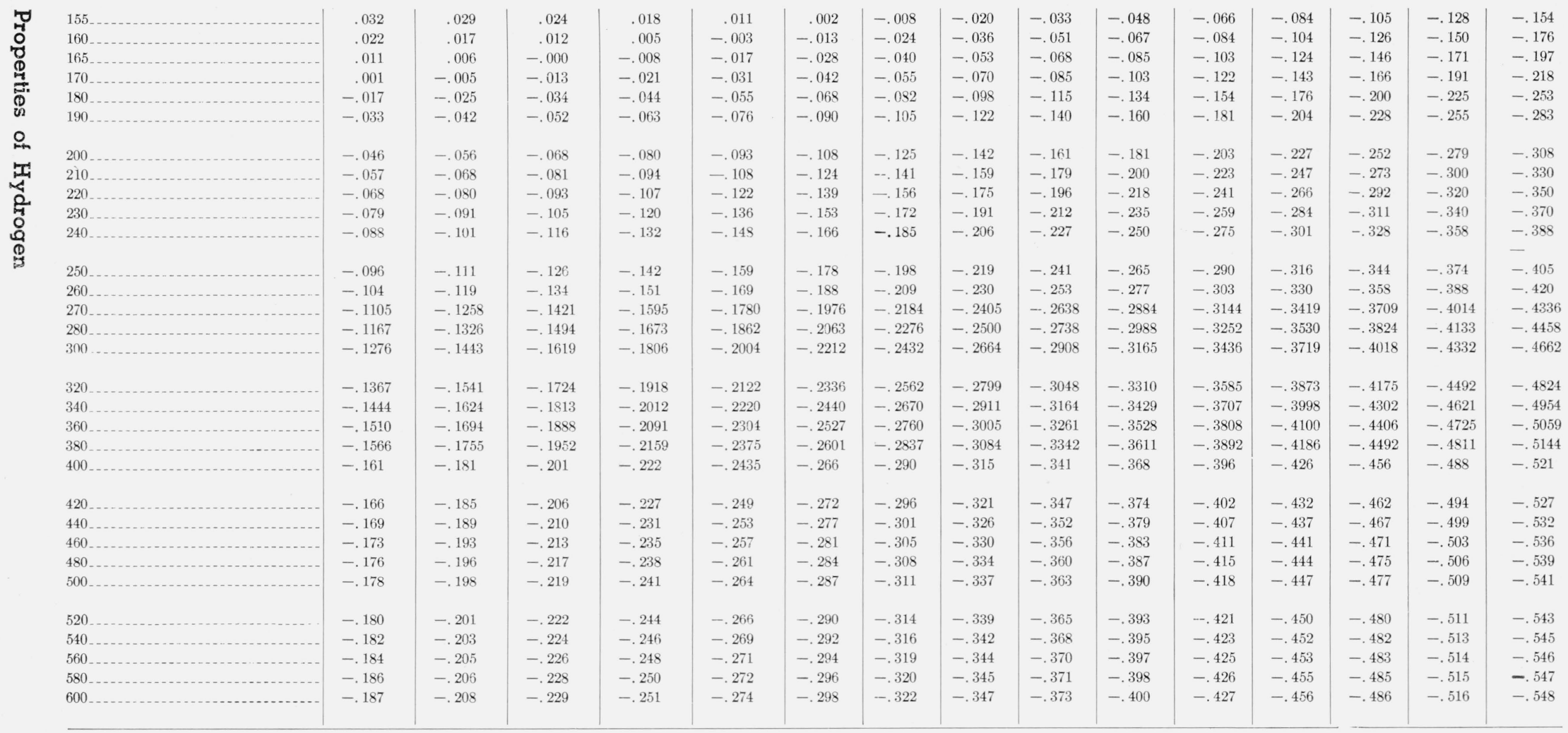


Values of $F / R T, E / R T$, and $\ln (f / P)$ may be obtained rather simply from values of $S / R$ and $H / R T$ and the $Z$-table in accordance with the following equations:

$$
\begin{gathered}
F / R T=(H / R T)-(S / R) \\
\ln \frac{f}{P}=\frac{F_{\rho, T \text { (real) }}-F_{\rho, T \text { (ideal) }}^{\circ}-\ln Z}{R T} \\
E / R T=(H / R T)-Z .
\end{gathered}
$$

The value of $\left[F_{\rho, T \text { (ideal) }}^{\circ}-F_{\rho, T \text { (reai) }} / R T\right]$ may be obtained by subtracting $\left(S^{\circ}-S\right) / R$, given in table 22 , from $\left(H^{\circ}-H\right) / R T$, given in table 23 .

The calculation of the heat capacities of the real gas involves the evaluation of

$$
\begin{gathered}
\int_{0}^{\rho}\left[T^{2}\left(d^{2} Z / d T^{2}\right)_{\rho} / \rho\right] d \rho . \\
{\left[C_{p(\text { real } \text { gas })}-C_{p(\text { (ideal) }}^{\circ}\right] / R=T\left\{\left(\frac{d}{d \rho}\left[\frac{H^{\circ}-H}{R T}\right]_{T}\left(\frac{d P}{d T}\right)_{\rho} /\left(\frac{d P}{d \rho}\right)_{T}-\left(\frac{d}{d T}\left[\frac{H^{\circ}-H}{R T}\right]\right)_{\rho}\right\}-\frac{H^{\circ}-H}{R T}\right.} \\
=-T\left(\frac{d}{d T}\left[\frac{H^{\circ}-H}{R T}\right]\right)_{\rho}-\frac{H^{\circ}-H}{R T}+\left[Z+T\left(\frac{d Z}{d T}\right)_{\rho}\right]\left[T\left(\frac{d Z}{d T}\right)_{\rho}-\rho\left(\frac{d Z}{d \rho}\right)_{T}\right] /\left[Z+\rho\left(\frac{d Z}{d \rho}\right)_{T}\right]
\end{gathered}
$$

The derivatives in eq 5.17 may be calculated from tables 14 and 23, using a method of tabular differentiation. Except for the first term, the derivatives in eq 5.17a are given in tables 15 and 17 .

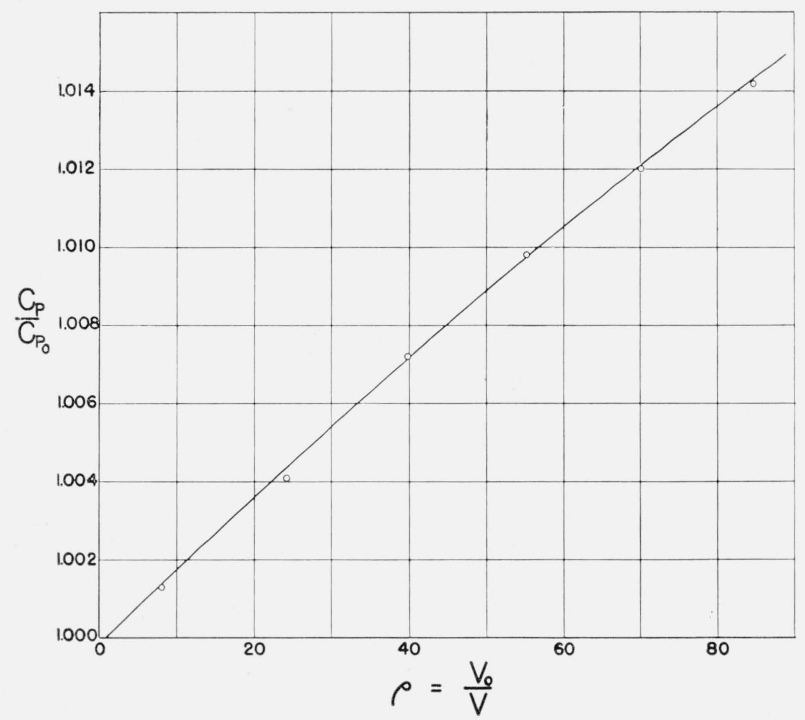

Figure 12. Effect of density on specific heat of $\mathrm{H}_{2}$ at $50^{\circ} \mathrm{C}$.

Figure 12 shows the dependence of the specific heat at constant pressure for hydrogen at $50^{\circ} \mathrm{C}$ upon the Amagat density $\rho$. The curve represents the results of the evaluation of formula 5.8, using
This may be carried out using the $\left(d^{2} Z / d T^{2}\right)_{\rho}$ table (table 16), and a method of tabular integration. Table 23 may be used to obtain $\int_{0}^{\rho}\left[T(d Z / d T)_{\rho} / \rho\right] d \rho$, since from eq 5.12 it follows that

$$
\begin{aligned}
& \int_{0}^{\rho}\left[T(d Z / d T)_{\rho} / \rho\right] d \rho= \\
& \frac{H^{\circ}-H}{R T}(\text { from table } 23)+(Z-1) .
\end{aligned}
$$

In the temperature and density ranges where $Z$ may be represented by an analytic expression, ${ }^{12}$ these two integrals may be evaluated by using series expansions for $Z$ and its derivatives in the integrands. The difference between the specific the PVT correlation of this paper. The plotted points are observations by Workman [49]. No other direct experimental data on the effect of pressure upon the specific heat at constant pressure are available for hydrogen.

An indirect indication of the effect of pressure on the specific heat of hydrogen is found in the work of van Itterbeek [78], who used the results of van Itterbeek and Keesom [77] on the effect of pressure on the velocity of sound in hydrogen at liquid hydrogen temperatures. The results of van Itterbeek at a pressure of one-tenth of an atmosphere indicate that the increase of $C_{p}$ with pressure above the zero-pressure value agrees with the PVT prediction within 3 percent at $17.5^{\circ} \mathrm{K}$ and at $19.0^{\circ} \mathrm{K}$, but is lower by more than 30 percent at $20.5^{\circ} \mathrm{K}$. At pressures above $1 / 2 \mathrm{~atm}$ at $20.5^{\circ} \mathrm{K}$, this difference in heat capacity has become approximately $0.1 \mathrm{cal} \mathrm{deg}^{-1} \mathrm{~mole}^{-1}$, but this discrepancy is reduced by roughly 50 percent if the data of van Itterbeek and Keesom are evaluated with values of $C_{p}-C_{v}$ based on the PV'T tables of this paper.

\footnotetext{
${ }_{12} \mathrm{Up}$ to $\rho=500$ at temperatures above $0^{\circ} \mathrm{C}$, the equation $Z=\exp \left(B \rho+C \rho^{2}\right)$ has been used. This is eq 4.8 and eq 4.9 is its series expansion. The symbols stand for functions of $T$, which are given by eq 4.11 and 4.12 .

From $\rho=0$ to $\rho=200$ and $T=14^{\circ}$ to $56^{\circ} \mathrm{K} Z$ can be expressed by $Z=1-$ $\left(A / T^{3 / 2}\right) \rho-\left(C / T^{3 / 2}\right) \rho^{2}$, which is equivalent to eq 4.14. The symbols $A$ and $C$ stand for functions of $T$, whose values are tabulated in table 19 .
} 
The specific heat of hydrogen at constant volume has been determined by Eucken [169] for various combinations of temperature and density in the ranges $35^{\circ}$ to $110^{\circ} \mathrm{K}$ and 60 to 150 Amagats.

Joule-Thomson coefficients of hydrogen may be of interest. These may be calculated from eq 4.6. For this calculation there are required: the value of $C_{p}$ which may be calculated using eq 5.8 or 5.17. Values of $Z,(d Z / d T)_{\rho}$, and $(d Z / d \rho)_{T}$ are given explicitly in tables 13,15 , and 17 . By using values of $C_{p}$ for $\mathrm{H}_{2}$ at $50^{\circ} \mathrm{C}$ derived from figure 12 , the following values of $\mu$ for $50^{\circ} \mathrm{C}$ were obtained by calculation: at $\rho=20, \quad \mu=-0.0350$ deg $\mathrm{atm}^{-1} ; \rho=40, \mu=-0.0364 ; \rho=60, \mu=-0.0378$; $\rho=80, \quad \mu=-0.0390$, and $\rho=100, \quad \mu=-0.0402$. By extrapolation, one obtains for $\mu$ at $\rho=0$ the value -0.0335 .

There are no accurate measured Joule-Thomson data for hydrogen for $50^{\circ} \mathrm{C}$ with which these calculated values of $\mu$ may be compared.

Results of measurements on Joule-Thomson effects in hydrogen and deuterium at liquid air and room temperatures have been published recently by Johnston and coworkers [57, 58], with curves showing calculated values for hydrogen based on the tables of this paper. ${ }^{*}$ Considering that the Joule-Thomson coefficients are not obtained with great simplicity from the PVT data and depend sensitively on the trends of the representation, the agreement is considered fairly satisfactory.

The location of the inversion curve for the Joule-Thomson effect in hydrogen on a $\rho$ - $T$ graph may be determined from tables 15 and 17 by finding values of $\rho$ and $T$ for which $T(d Z / d T)_{\rho}=$ $\rho(d Z / d \rho)_{T}$, in accordance with eq. 4.6.

An expression for $\mu$ in terms of derivatives of the enthalpy, $H$, is

$$
\mu=\frac{(d H / d \rho)_{T}}{\left(\frac{d H}{d \rho}\right)_{T}\left(\frac{d P}{d T}\right)_{\rho}-\left(\frac{d H}{d T}\right)_{\rho}\left(\frac{d P}{d \rho}\right)_{T}} .
$$

In accordance with this equation the inversion curve may be determined by inspection of the $\left(H^{\circ}-H\right) / R T$ table (table 23 ), since $\mu=0$ where

$$
\left(\frac{d\left(H^{\circ}-H\right) / R T}{d \rho}\right)_{T}=0 .
$$

${ }^{*}$ The tables of this paper were completed before the papers by Johnston and coworkers [57, 58] on the Joule-Thomson coefficients of $\mathrm{H}_{2}$ and $\mathrm{D}_{2}$ appeared. Our correlation of PVT data would doubtless have been better if these JouleThomson data had been available at the time the correlation was made.
The heavy curve in figure 13 is the inversion curve of hydrogen as given by the correlation of this paper. In locating it, values of $P$ were determined with the help of table 14. For temperatures below $75^{\circ} \mathrm{K}$ some extrapolation beyond the limit of the tables was necessary. In this extrapolated region the $\sigma$ versus $\rho$ diagram, figure 6 , was worked with, and the relation for the inversion curve on this diagram was used to get the extrapolated part of the inversion curve directly from the $\sigma$ versus $\rho$ diagram.

In a Joule-Thomson expansion of hydrogen at constant temperature from a high to a very low

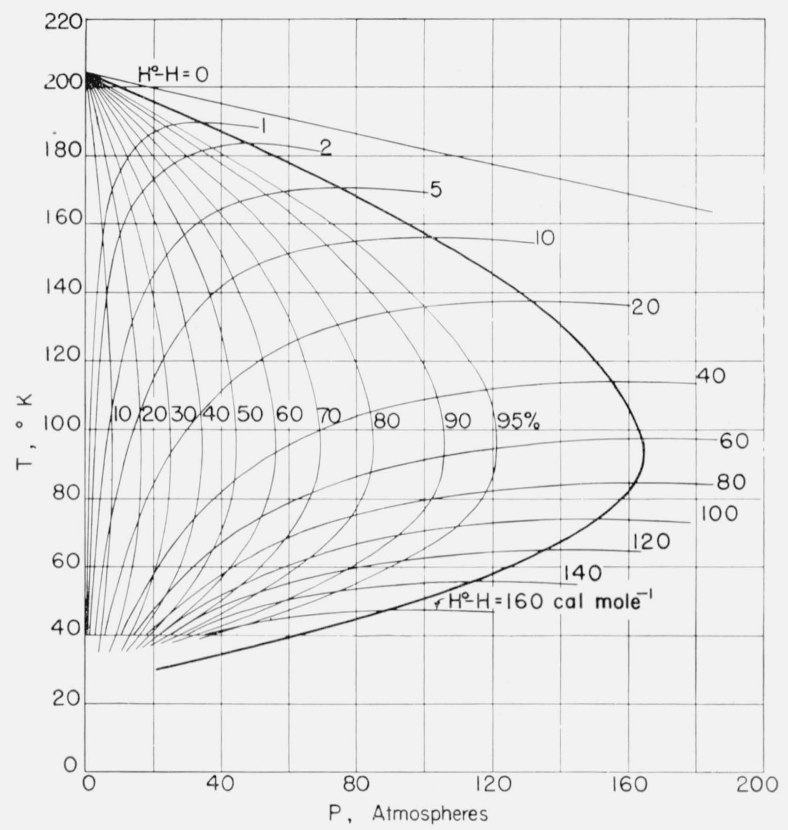

FIgURE 13. Curves related to the Joule-Thomson cooling of $\mathrm{H}_{2}$.

density, approaching zero density, there is a change in enthalpy equal to $\left(H^{\circ}-H\right)$. In figure 13 the curves that cross the inversion curve horizontally are curves of constant $H^{\circ}-H$. As $H^{\circ}$ is a function of temperature, these constant $\left(H^{\circ}-H\right)$ curves are not isenthalpics.

The horizontal crossing of the inversion curve by the $\left(H^{\circ}-H\right)$ curve is related to the fact that $\mu$, which is zero along the inversion curve, is equal to $(d H / d P)_{T} / C_{p}$, which means that along the inversion curve $(d H / d P)_{T}$ is zero. The enthalpy change $\left(H^{\circ}-H\right)$ is equal, very nearly, to the amount of refrigeration, per mole of gas, available for the liquefaction of hydrogen in a Hampson or Linde low pressure type of hydrogen liquefier in which a 
continuous flow of gaseous hydrogen is allowed to expand from a high to a low pressure without doing work against an external force system. The fraction $x$ of the high pressure hydrogen flow that might, theoretically, be liquefied is

$$
x=\frac{H^{\prime}-H}{H^{\prime}-H_{l i q}}=\frac{H^{\prime}-H}{\left(H^{\prime}-H_{v a p}\right)+L_{v}},
$$

where $H$ and $H^{\prime}$ are the enthalpies of the compressed and expanded hydrogen at the temperature at which the compressed hydrogen leaves the precooler and enters the last stage interchanger before expansion; $L_{v}$ is the heat of vaporization of liquid hydrogen at the boiling temperature determined by the pressure of the expanded hydrogen; and $\left(H_{v a p}-H_{l i g}\right)=L_{0}$ is the difference in enthalpies of saturated vapor and liquid in equilibrium at the pressure of the expanded hydrogen. Only a relatively small error is made in $x$ if in place of $H^{\prime}$ and $H_{v a p}$ for the real gas at atmospheric pressure one uses the enthalpies $H^{\circ}$ and $H^{\circ}{ }_{\text {rap }}$ of hydrogen in the ideal gas state at the same temperatures as would be used for $H^{\prime}$ and $H_{v a p}$.

$$
x=\frac{H^{\circ}-H}{H^{\circ}-H^{\circ}{ }_{v a p}+L_{v}} .
$$

For a temperature of precooling equal to $65^{\circ} \mathrm{K}$, the error introduced by the approximation is about 0.5 percent.

The lines of figure 13 that are roughly parallel to the inversion curve and converge with it at the inversion point, $204.6^{\circ} \mathrm{K}$, are lines showing the pressure at which $H^{\circ}-H$ has reached a given fraction of its maximum value for the given temperature. As the inversion curve is the line of maximum values of $\left(H^{\circ}-H\right)$ it is also the 100 -percent line in this family of constant percentage lines.

In the free expansion of a continuous flow of gas not doing work against an external force system, the maximum refrigeration is obtained by expanding from the inversion pressure for the given temperature of the compressed gas. The curves of constant percentage of maximum values of $\left(H^{\circ}-H\right)$ are also curves of constant percentage of the maximum available refrigeration in an expansion to low pressure.

Figure 13 makes apparent how greatly the refrigeration and the fraction of hydrogen liquefied (eq 5.21) by a Hampson type liquefier are increased by lowering the temperature of the compressed hydrogen before it enters the final interchanger from which expansion of the hydrogen takes place. It is also seen that the condition of highest inversion pressure $\left(92^{\circ} \mathrm{K}\right.$ and $\left.165 \mathrm{~atm}\right)$ is by no means the most favorable condition for liquefaction; a further cooling of the compressed hydrogen by 32 degrees nearly doubles the refrigeration produced and more than doubles the fraction liquefied. It is also seen from figure 13 that for the usual range of temperatures $\left(55^{\circ}\right.$ to $\left.90^{\circ} \mathrm{K}\right)$ to which compressed hydrogen is precooled before expansion in a Hampson-type liquefier, about 95 percent of the maximum refrigeration is obtained when the pressure of the compressed gas is only 75 percent of the inversion pressure.

\section{Viscosity and Thermal Conductivity}

\section{Viscosity and Thermal Conductivity of the Gas Near Atmospheric Pressure}

\section{(a) Hydrogen}

Values for the viscosity of gaseous normal hydrogen at atmospheric pressure for temperatures above the boiling point and at saturation pressure for two temperatures below the boiling point are given in table 24 . These were calculated using the empirical equation

$$
\eta=85.558 \times 10^{-7} \frac{T^{3 / 2}}{T+19.55} \frac{T+650.39}{(T+1175.9)} \text { poises }
$$

for the viscosity at very low pressure, ${ }^{13}$ together with values for the small differences between viscosities at atmospheric or saturation pressure and at very low pressure (see eq 6.17 and 6.16). The four constants of eq 6.1 were chosen on the basis of experimental data near $20^{\circ}, 90^{\circ}, 300^{\circ}$, and $685^{\circ} \mathrm{K}$. The value used for the viscosity of hydrogen at $685^{\circ} \mathrm{K}$ was 0.55 percent larger than the experimental values of Trautz and Zink [99], as their value was based on Millikan's value for the viscosity of air which is now known to be low by about this amount.

In figure 14 are plotted deviations of recent experimental viscosity data from eq 6.1. No changes were made in the experimental data for

13 This viscosity at very low pressure is a true or bulk viscosity. The pressure effect mentioned here is not the familiar low pressure effect on the apparent experimental viscosity involving the accommodation coefficient and the limited size of experimental apparatus. 
TABLE 24. Viscosity of gaseous hydrogen $\left(\mathrm{H}_{2}\right)$

\begin{tabular}{|c|c|c|c|c|c|}
\hline$T$ & $\eta$ & $T$ & $\eta$ & $T$ & $\eta$ \\
\hline${ }^{\circ} K$ & Poises & ${ }^{\circ} K$ & Poises & ${ }^{\circ} \mathrm{K}$ & Poises \\
\hline $10 \ldots$ & $51.0 \times 10^{-7}$ & $260 \ldots$ & $813.6 \times 10^{-7}$ & $620 \ldots$ & $1,461 \times 10^{-7}$ \\
\hline $20 \ldots$ & 109.3 & $270 \ldots$ & 834.6 & $640 \ldots$ & 1,493 \\
\hline $30 \ldots$ & 160.7 & $280 \ldots$. & 855.3 & $660 \ldots$ & 1,524 \\
\hline $40 \ldots$ & 206.8 & $290 \ldots$ & 875.8 & $680 \ldots$ & 1,555 \\
\hline $50 \ldots \ldots$ & 248.9 & $300 \ldots$ & 896.0 & $700 \ldots$ & 1,585 \\
\hline $60 \ldots$ & 287.6 & $310 \ldots$ & 916.0 & $720 \ldots$ & 1,616 \\
\hline $70 \ldots$ & 323.8 & $320 \ldots$ & 935.8 & $740 \ldots$ & 1,646 \\
\hline $80 \ldots$ & 357.9 & $330 \ldots$ & 955.4 & $760 \ldots$ & 1,675 \\
\hline $90 \ldots$ & 390.3 & $340 \ldots$ & 974.8 & $780 \ldots$ & 1,705 \\
\hline $100 \ldots$ & 421.1 & $350 \ldots$ & 994.0 & $800 \ldots$ & 1,734 \\
\hline $110 \ldots$ & 450.8 & $360 \ldots$ & 1,013 & $820 \ldots$ & 1,763 \\
\hline 120 & 479.3 & $370 \ldots$ & 1,032 & $840 \ldots$ & 1, 792 \\
\hline 130 & 507.0 & $380 \ldots$. & 1,051 & $860 \ldots$ & 1,820 \\
\hline $140 \ldots$ & 533.8 & $390 \ldots$ & 1,069 & $880 \ldots$ & 1,848 \\
\hline $150 \ldots$ & 559.8 & $400 \ldots$ & 1,087 & $900 \ldots$ & 1,876 \\
\hline $160 \ldots$ & 585.2 & $420 \ldots$ & 1,124 & $920 \ldots$ & 1,904 \\
\hline 170 & 610.0 & $440 \ldots$ & 1,160 & $940 \ldots \ldots$ & 1,932 \\
\hline $180 \ldots$ & 634.3 & $460 \ldots$ & 1,195 & $960 \ldots$ & 1,959 \\
\hline 190 & 658.1 & $480 \ldots$ & 1,230 & $980 \ldots$ & 1,986 \\
\hline $200 \ldots$ & 681.4 & $500 \ldots$ & 1,264 & 1,000 & 2,013 \\
\hline 210 & 704.3 & $520 \ldots$ & 1,298 & $1,020 \ldots$ & 2,040 \\
\hline $220 \ldots$ & 726.9 & 540 & 1,331 & 1,040 & 2,066 \\
\hline $230 \ldots$ & 749.0 & $560 \ldots$ & 1,364 & $1,060 \ldots$ & 2,092 \\
\hline $240 \ldots$ & 770.9 & $580 \ldots$ & 1,397 & $1,080 \ldots$ & 2,118 \\
\hline $250 \ldots$ & 792.4 & $600 \ldots$ & 1,429 & $1,100 \ldots$ & 2,144 \\
\hline
\end{tabular}

the differences in density. Deviations of table 24 values from eq 6.1 are represented in figure 14 by the peaked curve, which is appreciably above the zero line between $10^{\circ} \mathrm{K}$ and $100^{\circ} \mathrm{K}$ and in very close agreement with it at higher temperatures. This peaked curve represents the viscosity at atmospheric pressure above the boiling point and at saturation vapor pressure below the boiling point. Different reported values of viscosity at low temperatures are so poorly in agreement that their comparison does not indicate the magnitude of the peak, which has accordingly been obtained from theory, using data of state. To limit the crowding of experimental points in the figure, those plotted represent only data published since 1928, but a few data obtained after 1928 have been omitted. The data of Trautz and co-workers [94 to 102] would be in better agreement with the zero line if increased by about one half percent for the revision in the value for the viscosity of air.

It has been pointed out by others that the Sutherland formula

$$
\eta=\eta^{\prime}\left(\begin{array}{c}
T \\
T^{\prime}
\end{array}\right)^{3 / 2} \frac{T^{\prime}+C}{T+C}
$$

does not fit the data for hydrogen over an extended range of temperature. This may be seen in figure 14 in which the deviations of the Sutherland formula from eq 6.1 are represented by the curve below the zero line. The constant $C$ was evaluated at $300^{\circ} \mathrm{K}$ to represent the trend of the best data.

Values of the thermal conductivity of gaseous normal hydrogen are given in table 25 .

TABLE 25. Thermal conductivity of gaseous hydrogen at $1 \mathrm{~atm}$

\begin{tabular}{|c|c|c|c|}
\hline$T$ & $K$ & $T$ & $K$ \\
\hline${ }^{\circ} \mathrm{K}$ & $\begin{array}{c}\text { cal cm-1 } \\
\mathrm{sec}^{-1}{ }^{\circ} \mathrm{C}^{-1}\end{array}$ & ${ }^{\circ} K$ & $\begin{array}{c}\mathrm{cal} \mathrm{cm}-1 \\
\sec ^{-1}{ }^{\circ} \mathrm{C}^{-1}\end{array}$ \\
\hline $10 \ldots$ & $14.3 \times 10^{-6}$ & $260 \ldots$ & $397.0 \times 10^{-1}$ \\
\hline $20 \ldots$ & 34.6 & $270 \ldots$ & 409.7 \\
\hline $30 \ldots$ & 53.5 & 280 & 422.1 \\
\hline $40 \ldots$ & 70.7 & 290 & 434. 2 \\
\hline $50 \ldots$ & 86.5 & $300 \ldots$ & 446.3 \\
\hline $60 \ldots$ & 101.4 & $320 \ldots$ & 469.8 \\
\hline $70 \ldots$ & 116.1 & $340 \ldots$ & 492.8 \\
\hline $80 \ldots$. & 130.8 & $360 \ldots$ & 515 \\
\hline $90 \ldots$ & 145.9 & $380 \ldots$ & 537 \\
\hline $100 \ldots$ & 161.3 & $400 \ldots$ & 559 \\
\hline 110 & 177.0 & $420 \ldots$ & 580 \\
\hline $120 \ldots$ & 192.9 & 440 & 601 \\
\hline $130 \ldots$ & 208.8 & 460 & 622 \\
\hline $140 \ldots$ & 224.6 & 480 & 643 \\
\hline $150 \ldots$ & 240.4 & 500 & 664 \\
\hline $160 \ldots$ & 256.0 & 520 & 684 \\
\hline 170 & 271.4 & $540 \ldots$ & 705 \\
\hline $180 \ldots$ & 286.5 & $560 \ldots$ & 725 \\
\hline $190 \ldots$ & 301.1 & $580 \ldots$ & 745 \\
\hline $200 \ldots$ & 315.4 & $600 \ldots$ & 766 \\
\hline $210 \ldots$ & 329.6 & & \\
\hline $220 \ldots$ & 343.5 & & \\
\hline $230 \ldots$ & 357.2 & & \\
\hline $240 \ldots$ & 370.7 & & \\
\hline $250 \ldots$ & 384.0 & & \\
\hline
\end{tabular}

They were calculated from the equation

$$
\begin{aligned}
k= & {[1.8341-0.004458 T+(1.1308+} \\
& \left.0.0008973 T) C_{p}^{\circ}\right] \frac{\eta}{M} \frac{1}{\left(1+\frac{3.2}{T}\right)}
\end{aligned}
$$

In principle, a correction from low pressure to one atmosphere would be applicable, but it has been omitted because the uncertainty of the experimental values is much greater. In eq 6.3, $M$ is the molecular weight, $\eta$ the viscosity given by eq $6.1, C_{p}^{\circ}$ the specific heat in calories per mole per degree at constant pressure, and $T$ the temperature in degrees Kelvin. This equation is an empirical representation of the data and was 


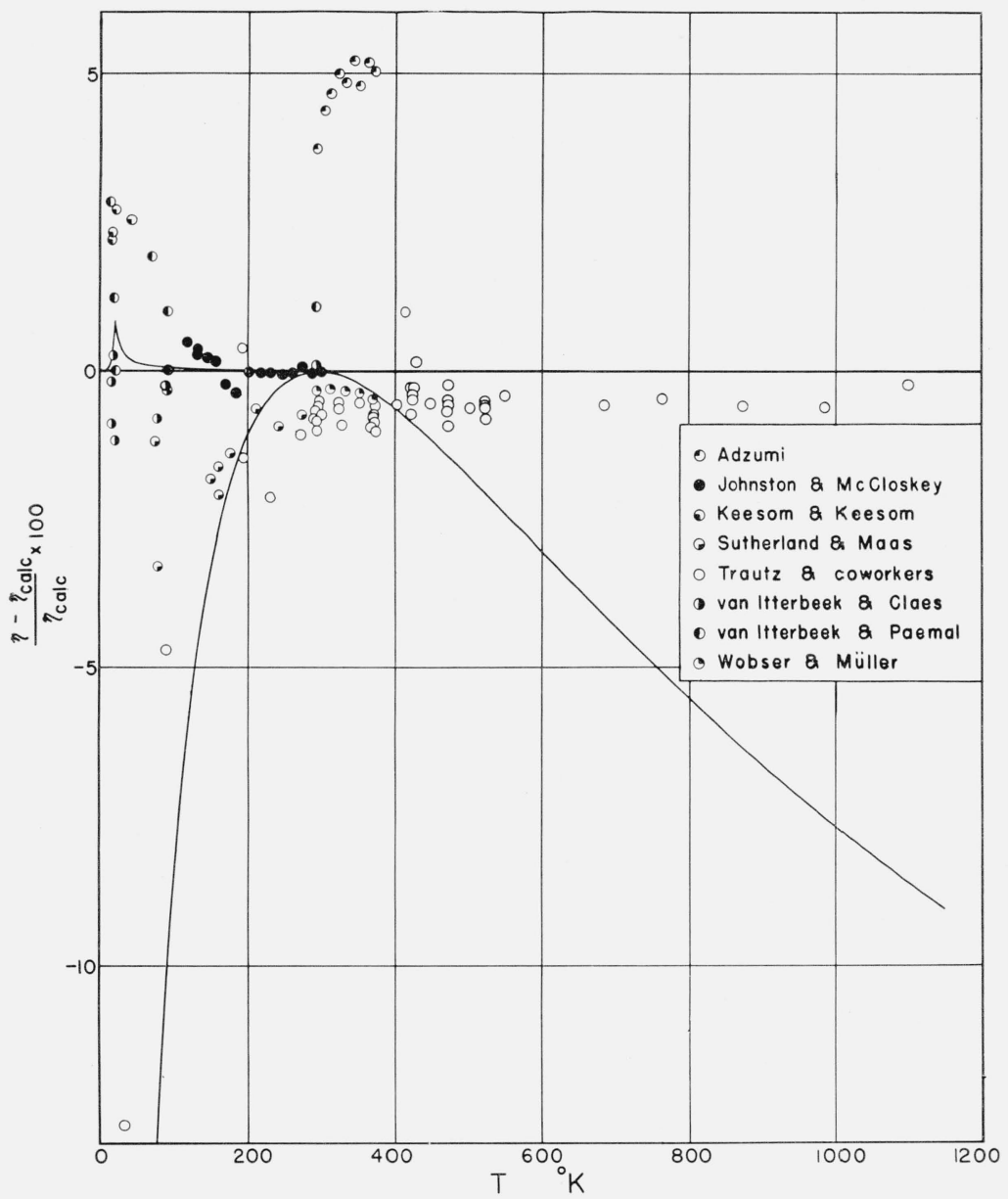

Figure 14. Viscosity of hydrogen.

obtained in several steps, which will be explained in the discussion that follows.

In figure 15 , curve $A$ represents eq 6.3 , whereas curves $B$ and $C$ are theoretical and are given for comparison. Curve $C$ is for Eucken's relation

$$
k=(9 \gamma-5) C_{o}^{\circ} \eta /(4 M),
$$

or its equivalent

$$
k=\left(C_{p}^{\circ}+1.25 R\right) \eta / M .
$$

Chapman and Cowling [137] proposed the formula

$$
k=\left[\frac{15}{4}(\gamma-1)+\frac{1}{2} U_{11}(5-3 \gamma)\right] \eta C_{v}^{\circ} / M,
$$

which is equivalent to

$$
k=\left[U_{11} C_{p}^{\circ}+\left(3.75-2.5 U_{11}\right) R\right] \eta / M .
$$

The transport of internal molecular energy of a gas is supposed to be represented better theoret- ically as a result of including the quantity $U_{11}$, which is the ratio of mean free path lengths for diffusion and viscosity.

$U_{11}$ is a pure number whose value was determined theoretically for (1) smooth elastic spheres and (2) for molecules repelling as the inverse fifth power of the distance (Maxwellian molecules), the values being 1.204 and 1.55 , respectively.

For $U_{11}$ equal to 1 , curve $C$ is obtained, as eq 6.6 and 6.7 then reduce to eq 6.4 and 6.5. Curve $B$ of figure 15 is a graph of eq 6.7 with $U_{11}=1.4$, a value indicated by a group of measurements of the conductivity near $300^{\circ} \mathrm{K}$. It is evident that the main body of the experimental data is not consistent with a constant value of $U_{11}$. On the basis of a value of 1.4 for $U_{11}$ near $300^{\circ} \mathrm{K}$ and a higher value at $700^{\circ} \mathrm{K}$, as indicated by a curve representing the data, the relation

$$
U_{11}=1.1308+0.0008973 T
$$




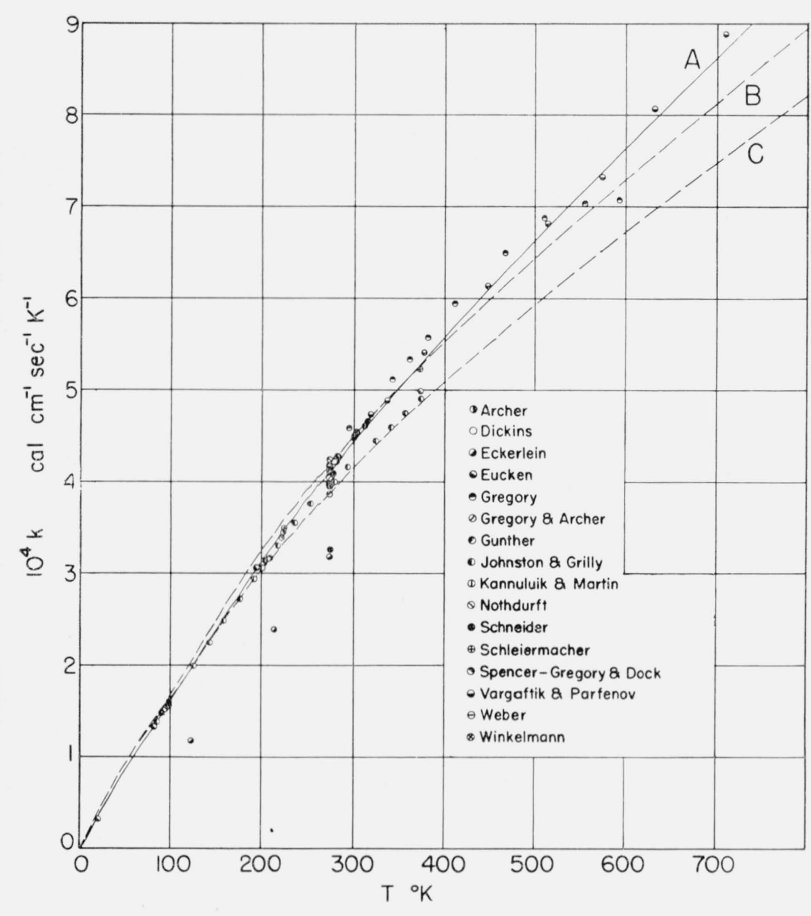

Figure 15. Thermal conductivity of hydrogen.

was adopted. It was found that the curve was not critically dependent on the functional form of $U_{11}$ as a change to $U_{11}=a+b \sqrt{T}$ altered the final curve negligibly between $300^{\circ}$ and $700^{\circ} \mathrm{K}$.

At temperatures somewhat below $100^{\circ} \mathrm{K}$, the ideal gas specific heat of hydrogen at constant pressure approaches the value $(5 / 2) R$ characteristic of a monatomic gas. For this value of $C_{p}^{\circ}$, the $U_{11}$ terms in eq 6.7 cancel and eq 6.4 to 6.7 reduce to

$$
k=\frac{5}{2} \eta\left(C_{v} / M\right)
$$

This equation has been derived exactly for a force that at all distances is repulsive and proportional to $1 / r^{5}$. Enskog [132] has shown that for attracting rigid spheres (Sutherland molecules),

$$
k=[2.522 / 1(1+0.03 C / T)] \eta C_{p} / M,
$$

where $C$ is the Sutherland constant in eq 6.2. Thermal conductivities of hydrogen measured at liquid air temperatures are a few percent lower than equations 6.4 to 6.9 would indicate. No theoretical explanation of this is at hand, but the agreement of the three independent investigations in this region indicates that the lower value is to be accepted. To take account of this, a correction factor $1 /(1+3.2 / T)$ has been included, having a form suggested by Enskog's theoretical result for attracting rigid spheres but with the constant chosen to fit these experimental data. The inclusion of this factor also brings the final curve closer to Eucken's experimental value at $20.96^{\circ} \mathrm{K}$, which is still almost 12 percent lower than the curve.

The curve as chosen to fit the thermal conductivity data is not regarded as completely satisfactory. In the temperature range $270^{\circ}$ to $400^{\circ} \mathrm{K}$, the experimental data appear to fall into two groups, one quite close to the curve adopted and the other lower by about 7 percent. The lower group includes the most recent data.

Equation 6.4 to 6.9 make it evident that at lowtemperatures where the specific heats of ortho and para hydrogen differ, their thermal conductivities differ also. This difference in thermal conductivity was the basis of the method of ortho-para analysis used by Bonhoeffer and Harteck [121]. The temperature or electrical resistance of an electrically heated wire carrying a given current determines, after calibration, the ortho-para composition of the hydrogen that surrounds the wire in a tube externally thermostated at liquid air temperature. A small difference is to be expected in the viscosities of ortho and para hydrogen by reason of small differences in their intermolecular forces manifested by small differences in vapor pressure, and density of the condensed states.

This difference in viscosities is small and was not detected in the experiment undertaken by Harteck and Schmidt [122], in which an accuracy of 1 percent was attained. In later developments of the so-called thermal conductivity method of ortho-para analysis, the pressure of the gas was reduced to make the mean free path large compared with the diameter of the heated wire. For this condition the ordinary thermal conductivity is not the controlling factor.

\section{(b) Deuterium}

Several investigations have been made of the viscosity of deuterium at atmospheric pressure, the most recent being that of Van Itterbeek and Van Paemel [106, 107], published in 1940. Table 26, which gives values for the ratio between viscosities of deuterium and hydrogen for several temperatures, was taken from the paper by Van Itterbeek and Van Paemel. 
TABLE 26. Ratio of viscosities for gaseous $\mathrm{D}_{2}$ and $\mathrm{H}_{2}$

\begin{tabular}{|c|c|}
\hline$T$ & $\eta\left(\mathrm{D}_{2}\right) / \eta\left(\mathrm{H}_{2}\right)$ \\
\hline${ }^{\circ} K$ & \\
\hline $293 \ldots \ldots$. & 1.40 \\
\hline $90 \ldots \ldots$ & 1. 38 \\
\hline $80 \ldots \ldots$ & 1.37 \\
\hline $70 \ldots \ldots$ & 1.36 \\
\hline $20 \ldots \ldots$ & 1. 24 \\
\hline $15 \ldots$ & 1. 24 \\
\hline $12.5 \ldots$ & 1. 24 \\
\hline
\end{tabular}

The ratio of the thermal conductivity of deuterium at $0^{\circ} \mathrm{C}$ to the thermal conductivity of hydrogen also at $0^{\circ}$ was determined by C. T. Archer [127] and by W. G. Kannuluik [130], who obtained respectively, the values $0.736_{5}$ and $0.732_{4}$. By using the mean of these values with appropriate values of $C_{p}$ and $\eta$, one obtains for $U_{11}$ in eq 6.7 for the thermal conductivity of $\mathrm{D}_{2}$ at $0^{\circ} \mathrm{C}$ the value 1.55. Archer also measured the thermal conductivity of various equilibrium mixtures of $\mathrm{H}_{2}, \mathrm{HD}$, and $\mathrm{D}_{2}$.

For two isotopic gases with identically the same intermolecular forces, the classical theory values for the ratio of their viscosities, and the ratio of their thermal conductivities at temperatures where their heat capacities are equal are

$$
\eta_{1} / \eta_{2}=\sqrt{M_{1} / M_{2}} \text { and } k_{1} / k_{2}=\sqrt{M_{2} / M_{1}}
$$

For $\mathrm{H}_{2}$ and $\mathrm{D}_{2}$ these ratios have the values: $\eta_{\mathrm{D}_{2}} / \eta_{\mathrm{H}_{2}}=1.414$ and $k_{\mathrm{D}_{2}} / k_{\mathrm{H}_{2}}=0.707$, and are independent of the intermolecular force field so long as it is the same for the two isotopes. The difference between the rotational heat capacities of $\mathrm{H}_{2}$ and $\mathrm{D}_{2}$ at low temperatures by itself makes the ratio $k_{\mathrm{D}_{2}} / k_{\mathrm{H}_{2}}$ larger and thus has an effect opposite to but less than that of the smaller mean velocity of $\mathrm{D}_{2}$ molecules caused by the greater mass. Using Eucken's eq 6.4 for $k$ and making allowance for the difference in heat capacities of $\mathrm{H}_{2}$ and $\mathrm{D}_{2}$, one obtains 0.718 for $k_{\mathrm{D}_{2}} / k_{\mathrm{H}_{2}}$ at $0^{\circ} \mathrm{C}$. The classical theory values for these ratios of thermal conductivities and viscosities are approached closely at room temperatures. The effect of quantum mechanical interaction in transport phenomena can be described in terms of increase in the apparent size of the molecules. In classical theory the size of the molecule plays an important role, the viscosity and thermal conductivity decreasing as the size increases. For hydrogen and deuterium, the quantum mechanical increase in apparent size is small at room temperature but becomes large at low temperature. The increase depends also upon the masses of the colliding molecules and is larger for $\mathrm{H}_{2}$ than for $\mathrm{D}_{2}$ at the same temperature. It was pointed out in the section on the PVT data for deuterium that the quantum theory of second virial coefficients includes an effect interpretable classically as an increase in apparent size of molecules, becoming very large at low temperatures. The quantum mechanically obtained increase in apparent size with lowering of temperature is not the same for viscosity as that associated with the second virial coefficient, however. This is not surprising when one considers that the increase in the mean de Broglie wave length with decreasing temperature increases the diffraction behind a scattering molecule; an effect that does not enter in the determination of the second virial coefficient, but which taken by itself would decrease the apparent size of a scattering molecule for viscosity.

\section{Viscosity and Thermal Conductivity of the Gas at High Pressures}

There are no experimental data on the thermal conductivity of gaseous $\mathrm{H}_{2}$ at high pressures. For viscosity, however, experimental data obtained by Boyd [134] and Gibson [135] are available. Gibson's data, which are for $25^{\circ} \mathrm{C}$, are more precise than those of Boyd and are plotted in figure 16. It will be seen that there is fairly good agreement between these better experimental data and the curve representing the theoretical formula due to Enskog. Differing approaches to the problem of relating viscosity and variables of state will be found elsewhere [133, 136].

In elementary theory, the viscosity and thermal conductivity for a given gas are proportional to the product of $V, \rho$, and $\Lambda$, where $V$ is the mean molecular velocity, $\rho$ is the density, and $\Lambda$ is a suitable mean path length for the transfer of momentum or energy. Although $\Lambda$ is often taken as identical with the ordinary free path of molecular motion, it is actually greater by a small distance of the order of magnitude of a molecular diameter, as at each collision the momenta and energies are transferred an additional distance related to the diameters of the molecules involved. Thus instead of $\Lambda$ decreasing as $1 / \rho$ when $\rho$ is increased, which would make $\rho \Lambda$ independent of 
$\rho, \Lambda$ decreases a little less slowly so as to make $\rho \Lambda$ increase slightly as $\rho$ is increased. Accordingly, both the thermal conductivity and the viscosity of a gas would be expected to increase with increasing density, particularly when multiple encounters between molecules occur frequently as in the case of high densities.

Enskog's theory was developed for a gas whose molecules were assumed to be mutually attracting rigid spheres, for which the equation of state has the form

$$
P+a \rho^{2}=R T \rho(1+b \rho \chi),
$$

used by Enskog takes account of simultaneous encounters of three and four molecules as treated by Boltzmann and Clausius.

According to Enskog's theory, the viscosity and thermal conductivity of a compressed gas are related to the viscosity $\eta_{0}$ and conductivity $k_{0}$ at low pressure by the equations

$$
\eta / \eta_{0}=b \rho[1 /(b \rho \chi)+0.8+0.7614 b \rho \chi \ldots]
$$

and

$$
k / k_{0}=b \rho[1 /(b \rho \chi)+1.2+0.7574 b \rho \chi \ldots]
$$

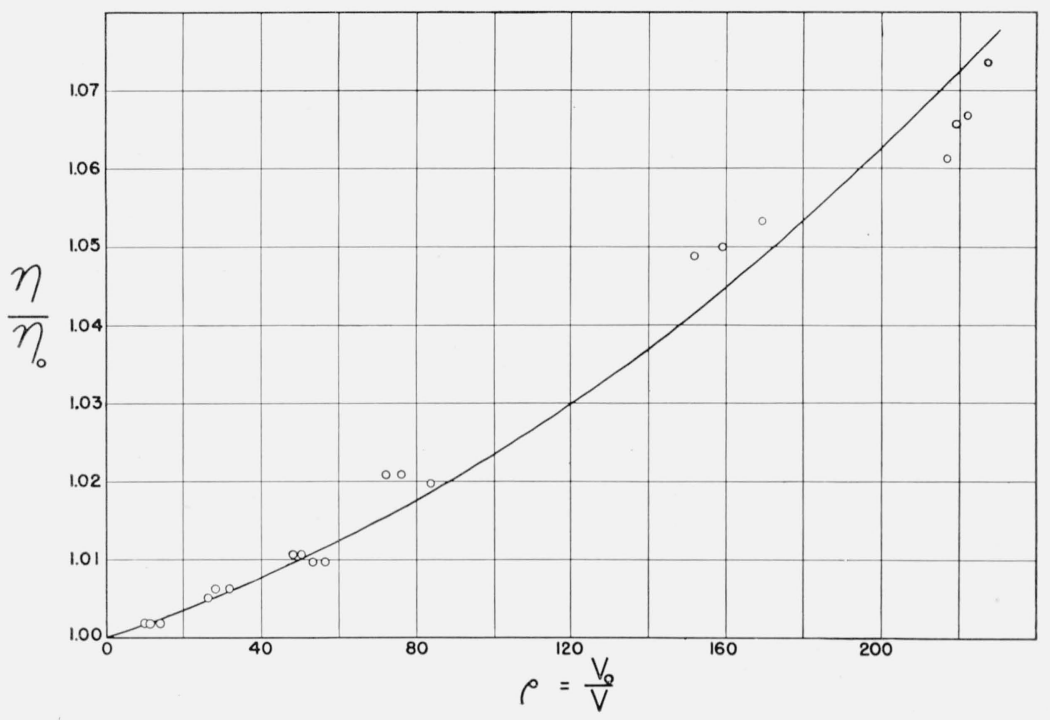

Figure 16. Effect of density on viscosity of hydrogen at $25^{\circ} \mathrm{C}$.

where the constants $a$ and $b$ are assumed to be independent of $T$ and $\rho$, and $\chi$ is a function of $\rho$ expressed in the form of a power series in $b \rho$. The equation of state that was used is thus almost the same as the Van der Waals equation

$$
\begin{aligned}
P+a \rho^{2}= & R T \rho(1-b \rho)^{-1}=R T \rho \\
& {\left[1+b \rho\left(1+b \rho+b^{2} \rho^{2}+\ldots\right)\right] }
\end{aligned}
$$

except for the details of the dependence of $\chi$ upon $\rho$. The Van der Waals equation is derived on the basis that simultaneous encounters of three or more molecules are rare enough to be neglected. Only at low pressures is this valid and under this condition terms of the second degree and higher in $b \rho$ are neglected in the derivation. The function

$$
\chi=1+0.625 b \rho+0.2869 b^{2} \rho^{2}+\ldots .
$$

It follows from eq 6.11, the equation of state assumed for Enskog's theory, that

$$
b \rho \chi=\frac{T}{P}\left(\frac{d P}{d T}\right)_{\rho}\left(\frac{P V}{R T}\right)-1=Z-1+T\left(\frac{d Z}{d T}\right)_{\rho} .
$$

Thus, the value of $b \rho \chi$ may be calculated from the tables of $Z$ and $(d Z / d T)_{\rho}$ and the value of $b \rho$ may then be found with the help of eq 6.13.

Over the range of Gibson's experimental viscosity data very little change is made in the values predicted if simple power series expansions in $b \rho \chi$, obtained from equations 6.14 and 6.15 , are used:

$$
\begin{array}{r}
\eta / \eta_{0}=1+0.175 b \rho \chi+0.7557(b \rho \chi)^{2}-0.405(b \rho \chi)^{3} \\
k / k_{0}=1+0.575 b \rho \chi+0.5017(b \rho \chi)^{2}-0.204(b \rho \chi)^{3}
\end{array}
$$

\section{Properties of Hydrogen}


The coefficient of the last term of each equation would be changed if higher order terms were added to eq $6.13,6.14$, and 6.15 . Dropping the last term of eq 6.17 for $\eta / \eta_{0}$ does not significantly change the agreement with Gibson's experimental data.

In order to show the general magnitude of the theoretical effect of pressure on the viscosity and thermal conductivity of hydrogen the preceding equations have been evaluated for several additional combinations of temperature and pressure, using data from the PV'T tables. Table 27 gives the values thus obtained. It is seen that the calculated relative change in $\eta$ and $k$ with pressure is much more pronounced at the lower temperatures, for which large deviations from the ideal gas law occur even at moderate pressures.

\section{TABLE 27. Effect of pressure on viscosity and thermal} conductivity of hydrogen

\begin{tabular}{|c|c|c|c|}
\hline$T$ & $P$ & $\eta / \eta_{0}$ & $k / k_{0}$ \\
\hline${ }^{\circ} \mathrm{K}$ & atm & & \\
\hline $18 \ldots$ & 0.455 & 1. 0045 & 1. 0138 \\
\hline $20 \ldots \ldots$ & .889 & 1. 0077 & 1. 0225 \\
\hline $22 \ldots$ & 1. 565 & 1. 0126 & 1. 0347 \\
\hline $30 \ldots$ & 1 & 1. 0037 & 1. 0114 \\
\hline $30 \ldots$ & 2.04 & 1. 0086 & 1. 0248 \\
\hline $38 \ldots$ & 30.4 & 1.53 & 1. 76 \\
\hline $40 \ldots$ & 1 & 1. 0021 & 1.0068 \\
\hline $40 \ldots$ & 2.80 & 1. 0067 & 1. 0199 \\
\hline $40 \ldots$ & 37.2 & 1.53 & 1.76 \\
\hline $50 \ldots$ & 1 & 1. 0015 & 1. 0048 \\
\hline $50 \ldots$ & 3.55 & 1. 0060 & 1.0178 \\
\hline $50 \ldots$ & 50 & 1.31 & 1. 49 \\
\hline $60 \ldots$ & 1 & 1. 0012 & 1. 0037 \\
\hline $70 \ldots$ & 1 & 1. 0009 & 1. 0030 \\
\hline $70 \ldots$ & 5. 06 & 1. 0051 & 1.0155 \\
\hline $70 \ldots$ & 50 & 1. 11 & 1. 22 \\
\hline $80 \ldots$ & 1 & 1. 00075 & 1. 0024 \\
\hline $90 \ldots$ & 1 & 1. 00065 & 1. 0021 \\
\hline $90 \ldots$ & 6. 56 & 1. 0047 & 1. 0141 \\
\hline $90 \ldots$ & 50.0 & 1. 06 & 1. 13 \\
\hline $100 \ldots$ & 1 & 1. 00056 & 1.0018 \\
\hline 110 & 1 & 1. 00049 & 1. 0016 \\
\hline $150 \ldots$ & 1 & 1. 00034 & 1. 0011 \\
\hline $250 \ldots$ & 1 & 1. 00018 & 1. 0006 \\
\hline 400 & 1 & 1. 00010 & 1. 0003 \\
\hline $600 \ldots$ & 1 & 1. 00006 & 1. 0002 \\
\hline
\end{tabular}

\section{The Viscosity of Liquid Hydrogen}

The first determination of the viscosity of liquid hydrogen was made in 1917 by Verschaffelt and Nicaise [138] from measurements of the logarithmic decrement of the oscillatory rotation of a sphere in liquid hydrogen at $20.36^{\circ} \mathrm{K}$.
Later, determinations were made of the viscosity of liquid hydrogen from $15^{\circ}$ to $20^{\circ} \mathrm{K}$, in 1938 by Keesom and Mac Wood [139] from measurements of the logarithmic decrement of an oscillating disc, and in 1939 by Johns [140], using the capillary flow method. The reported viscosities are

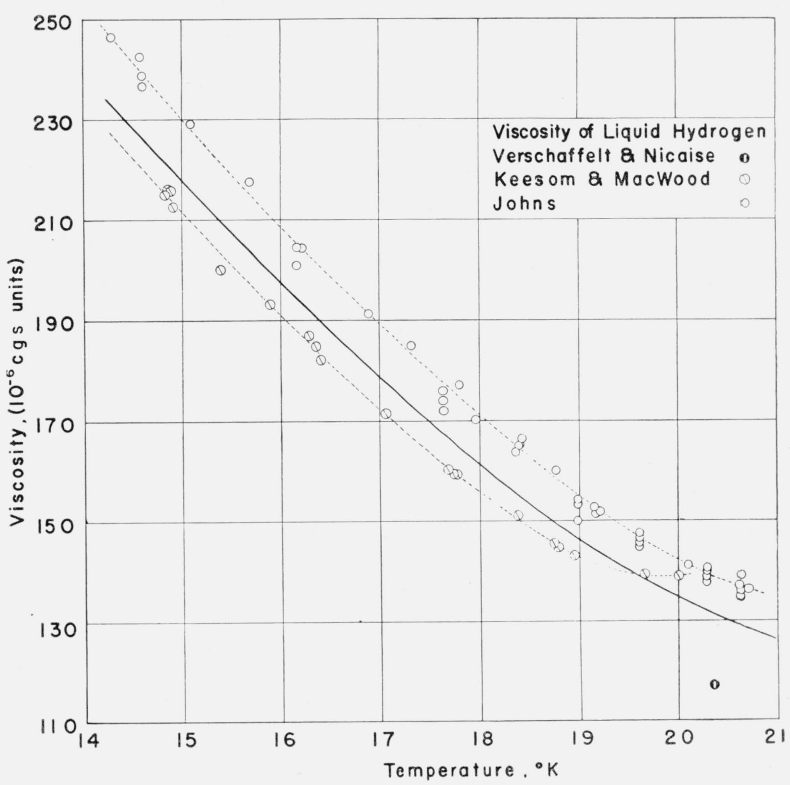

Figure 17. Viscosity of liquid hydrogen.

shown in figure 17. The values obtained by Johns are roughly 10 percent greater than those of Keesom and Mac Wood except near the boiling point, $20.4^{\circ} \mathrm{K}$. There seems to be no clear indication in the papers reporting the measurements that either of these two later sets is less dependable than the other. Accordingly a curve to represent the present most probable values of the viscosity of liquid hydrogen was drawn principally between the two sets. Near the boiling point the curve was drawn approximately parallel to that of Johns because it was felt that the lower value of Verschaffelt and Nicaise supported the more regular variation of viscosity with temperature as reported by Johns.

\section{Pressure Temperature Relations for Two-Phase Equilibria for $\mathrm{H}_{2}, \mathrm{HD}$, and $\mathrm{D}_{2}$ as Single Components}

In this section are presented data on (1) vapor pressures of solid and liquid $\mathrm{H}_{2}, \mathrm{HD}$, and $\mathrm{D}_{2}$ with such derived constants as normal boiling temperatures and triple-point temperatures and pres- 
sures; differences between the vapor pressures of different mixtures of $o$ - and $p-\mathrm{H}_{2}$; and changes in vapor pressures of ortho-para $\mathrm{H}_{2}$ mixtures resulting from self conversion; (2) the pressure-temperature relations for the solid-liquid equilibrium of $\mathrm{H}_{2}, \mathrm{HD}$, and $\mathrm{D}_{2}$. The data are presented in the form of equations, tables, and graphs.

\section{Vapor Pressures, Boiling, and Triple Points ${ }^{14}$}

The present vapor-pressure data on the hydrogens can be fitted with equations of the form

$$
\log _{10} P=A+B / T+C T
$$

to within the accuracy of the experimental data. The millimeter of $\mathrm{Hg}$ at $0^{\circ} \mathrm{C}$ and standard gravity

${ }_{14}$ Boiling-point and triple-point data from this section have been used in advance of publication in the "Tables of Selected Values of Chemical Thermodynamic Properties" prepared by the National Bureau of Standards in conjunction with the Office of Naval Research of the U. S. Navy Department. is used in this section as the unit of vapor pressure. Temperatures are on the Kelvin Scale.

In tables 28 and 29 the vapor pressures, boiling points, and triple points of the different isotopic and ortho-para modifications of hydrogen are compared.

\section{(a) $\mathrm{H}_{2}$}

The differences between the hydrogen vaporpressure data reported in the literature [143 to $146,148]$ are the result, principally, of differences in the temperature scales used by different observers and of unknown differences in the ortho-para composition of the hydrogen.

The vapor-pressure data recently obtained [146] at the National Bureau of Standards are on the low-temperature scale established at the National Bureau of Standards and are for known ortho-

TABLE 28. Vapor pressures of the several isotopic varieties of hydrogen at integral temperatures and at their triple points and boiling points.

[Values marked $(*)$ were obtained by extrapolation of the vapor-pressure equation to temperatures at which no data were available. The 0 - $\mathrm{H}_{2}$ table is based on an extrapolation with respect to composition.]

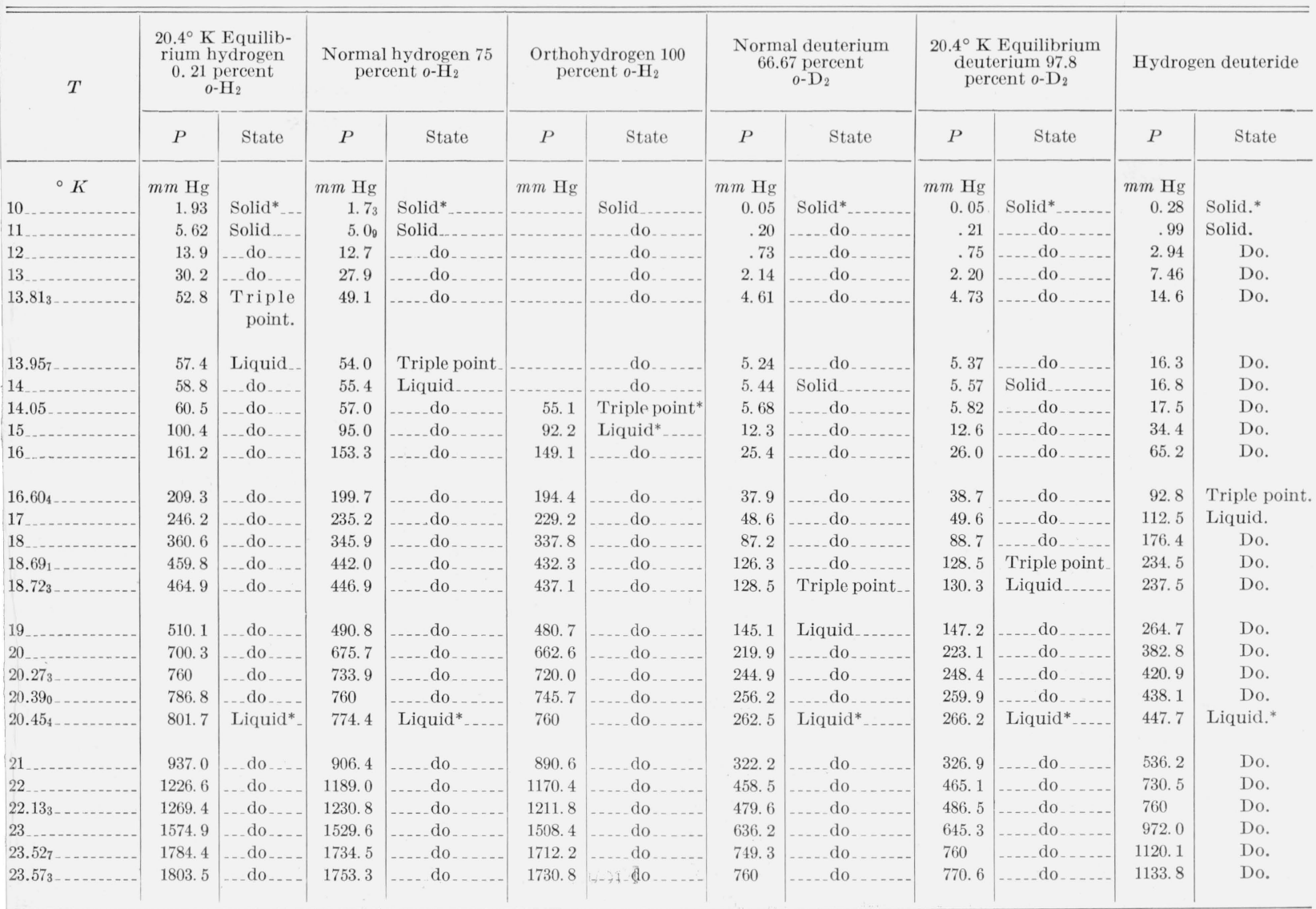


TABLE 29. Boiling points and triple points of the hydrogens

\begin{tabular}{|c|c|c|c|}
\hline & \multirow{2}{*}{$\begin{array}{l}\text { Boiling } \\
\text { point }\end{array}$} & \multicolumn{2}{|c|}{ Triple point } \\
\hline & & $T$ & $P$ \\
\hline & ${ }^{\circ} K$ & ${ }^{\circ} K$ & $m m \mathrm{Hg}$ \\
\hline & $20.27_{3}$ & $13.81_{3}$ & 52.8 \\
\hline 38 percent $0-\mathrm{H}_{2}, 62$ percent $p-\mathrm{H}_{2}$ & 20.32 & 13.86 & 53.0 \\
\hline Normal hydrogen $\left(75 \% 0-\mathrm{H}_{2}\right) \ldots \ldots$ & 20. $39_{0}$ & $13.95_{7}$ & 54.0 \\
\hline Orthohydrogen & 20.45 & 14. 05 & 55.1 \\
\hline Normal deuterium $\left(66.67 \% 0-\mathrm{D}_{2}\right) \ldots$ & $23.57_{3}$ & 18. $72_{3}$ & 128.5 \\
\hline $20.4^{\circ} \mathrm{K}$ equilibrium deuterium $\left(97.8 \% 0-\mathrm{D}_{2}\right)$ & 23.527 & 18. $69_{1}$ & 128.5 \\
\hline Paradeuterium & 23. 66 & 18. 78 & 128.5 \\
\hline Hydrogen deuteride. & 22.133 & $16.60_{4}$ & 92.8 \\
\hline
\end{tabular}

para compositions. Only the NBS results are given here.

Normal hydrogen (75 percent $0-\mathrm{H}_{2}, 25$ percent $\left.p-\mathrm{H}_{2}\right)$ :

Liquid: $\log _{10} P(\mathrm{~mm} \mathrm{Hg})=4.66687-$

$\frac{44.9569}{T}+0.020537 T$.

Solid: $\log _{10} P(\mathrm{~mm} \mathrm{Hg})=4.56488-$

$\frac{47.2059}{T}+0.03939 T$.

20.4 $4^{\circ} \mathrm{K}$-equilibrium hydrogen (99.79 percent $p$ - $\mathrm{H}_{2}$, 0.21 percent $o-\mathrm{H}_{2}$ :

Liquid: $\log _{10} P(\mathrm{~mm} \mathrm{Hg})=4.64392-$

$\frac{44.3450}{T}+0.02093 T$.

Solid: $\log _{10} P(\mathrm{~mm} \mathrm{Hg})=4.62438-$

$\frac{47.0172}{T}+0.03635 T$.

The triple-point temperatures and pressures were determined experimentally with a low-temperature calorimeter with a platinum resistance thermometer for the temperature measurements. Equations 7.2 to 7.5 were made to fit these triple points, and are based on vapor pressure data extending from $10.5^{\circ}$ to $20.4^{\circ} \mathrm{K}$. Although the equation for liquid normal $\mathrm{H}_{2}$ is based only on National Bureau of Standards data below $20.4^{\circ} \mathrm{K}$, the equation represents, within the limits of experimental accuracy, the Leiden data that extend nearly to the critical point, $33.19^{\circ} \mathrm{K}$. As mentioned in section IV, the vapor-pressure equation for normal hydrogen was used in constructing the PV'T relations for hydrogen. The experimentally determined triple-point temperatures and pres- sures for $n-\mathrm{H}_{2}$ and $e-\mathrm{H}_{2}$ are given in tables 28 and 29.

Figure 18 is a diagram of differences between the vapor pressures of a $20.4^{\circ} \mathrm{K}$ equilibrium mixture of 0 - and $p-\mathrm{H}_{2}\left(0.21\right.$ percent $\left.o-\mathrm{H}_{2}\right)$ and five different mixtures of $o$ - and $p-\mathrm{H}_{2}$ in the liquid state. The vapor pressure of the $20.4^{\circ} \mathrm{K}$ equilibrium mixture is denoted by $P_{\left(e-\mathrm{H}_{2}\right)}$ and that of any other mixture by $P_{\text {(mixure) }}$. Each curve of the graph is for a single mixture whose composition is indicated on the graph by its $o-\mathrm{H}_{2}$ composition. The 75 percent curve is for normal hydrogen. The vapor pressure differences $\Delta P$ are plotted as a function of the vapor pressure of the $20.4^{\circ} \mathrm{K}$ equilibrium hydrogen. The circles represent the experimental data.

Figure 19 shows the vapor pressure differences of figure 18 extended into the solid range, for mixtures of 38 and 75 percent ortho composition. At the extreme right of the figure, these mixtures and the $e-\mathrm{H}_{2}$ with which they are compared are all liquid. Passing to the left, the first sharp break encountered on either curve corresponds to the triple point of the mixture. The second sharp break corresponds to the triple point of $e-\mathrm{H}_{2}$. To the left of the last break, both materials are solid. Between the two breaks on either curve, the mixture is solid but the $e-\mathrm{H}_{2}$ is liquid.

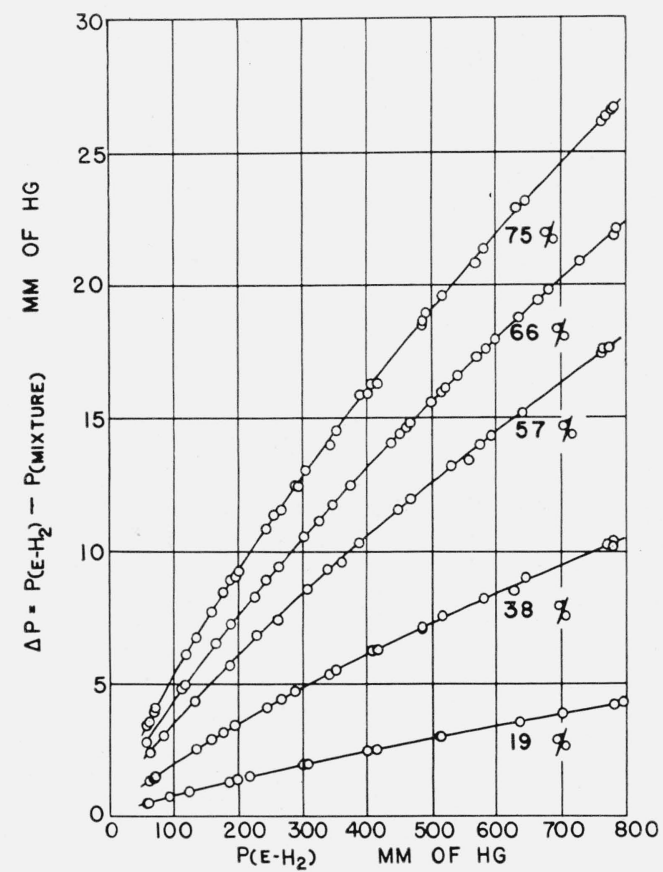

FIGURE 18. Vapor pressure differences for liquid ortho-para $\mathrm{H}_{2}$ mixtures. 


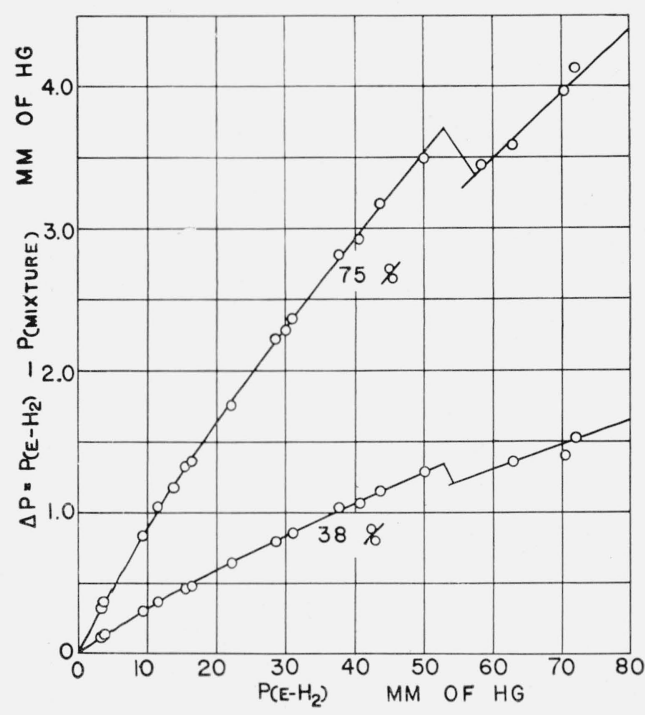

Figure 19. Vapor pressure differences for solid ortho-para $\mathrm{H}_{2}$ mixtures.

A comparison of the $\Delta P$ 's for different mixtures of 0 - and $p-\mathrm{H}_{2}$ in figures 18 and 19 shows that the $\Delta P$ 's are not proportional to their corresponding differences in composition.

For ideal solutions the ratio $\Delta P / \Delta x$, where $\Delta x$ is the difference in composition, is independent of the composition at constant temperature. In figure 20 this ratio is plotted for four temperatures, the circles representing the experimental vapor pressure data as given by points on the smooth curves of figure 18. Figure 20 shows that the vapor pressures of ortho-para mixtures differ greatly from ideal solution predictions.

The vapor pressure differences $\left(P_{e-H_{2}}-P_{m}\right)$ for mixtures of 0 - and $p-\mathrm{H}_{2}$ of any composition at $14.00^{\circ}, 16.00^{\circ}, 18.00^{\circ}$ and $20.39^{\circ} \mathrm{K}$ may be calcuated from the isotherms of figure 20. Other sotherms may be determined with the help of igures 18 and 19. By extending the isotherms of figure 20 to 100 percent $o-\mathrm{H}_{2}$, the vapor pressure of pure liquid $o-\mathrm{H}_{2}$ was determined. The following equation represents the vapor pressures of pure liquid $0-\mathrm{H}_{2}$ obtained in this way:

liquid: $\log _{10} P(\mathrm{~mm} \mathrm{Hg})=4.65009-\frac{45.0439}{T}+$

$$
0.021168 T
$$

The triple-point temperature and pressure of ) $-\mathrm{H}_{2}$ were determined by a quadratic extrapolation of the triple point temperatures and pressures of
$e-\mathrm{H}_{2}\left(20.4^{\circ} \mathrm{K}\right), m-\mathrm{H}_{2}\left(38\right.$ percent $\left.o-\mathrm{H}_{2}\right)$ and $n-\mathrm{H}_{2}$. The values thus obtamed for $0-\mathrm{H}_{2}$ were $14.05^{\circ}$ $\mathrm{K}$ and $55.1 \mathrm{~mm} \mathrm{Hg}$. These are in agreement with eq 7.6 for the vapor pressure of liquid $o-\mathrm{H}_{2}$.

If linear extrapolation is used, omitting the values for $m-\mathrm{H}_{2}$, one obtains $14.00^{\circ} \mathrm{K}$ and $54.4 \mathrm{~mm}$ $\mathrm{Hg}$ as lower limiting values of the triple point constants for $o-\mathrm{H}_{2}$. The triple point constants of $m-\mathrm{H}_{2}$ were obtained by reading the values $P\left(e-\mathrm{H}_{2}\right)$ and $\Delta P$ corresponding to the upper break in the 38 percent curve. The difference of these is the triple point pressure of $m-\mathrm{H}_{2}$. By substituting $P\left(\mathrm{e}-\mathrm{H}_{2}\right)$ into the vapor pressure equation (eq 7.4) for liquid $e-\mathrm{H}_{2}$, the triple point temperature of $m-\mathrm{H}_{2}$ is obtained. The uncertainties in these derived triple point constants of $m-\mathrm{H}_{2}$ and $o-\mathrm{H}_{2}$ are greater than for the experimentally determined values for $e-\mathrm{H}_{2}$ and $n-\mathrm{H}_{2}$.

The vapor pressure of a nonequilibrium mixture of $o-$ and $p-\mathrm{H}_{2}$ changes slowly with time because of the slow conversion of a nonequilibrium mixture, liquid or solid, to the equilibrium composition. At its normal boiling point, the vapor pressure of $n-\mathrm{H}_{2}$ changes at the rate of $0.23 \mathrm{~mm} \mathrm{Hg}$ per hour [148]. Paramagnetic substances increase the rate of conversion. The rate of increase of the vapor pressure at $20.4^{\circ} \mathrm{K}$ of a sample of hydrogen containing 0.01 percent oxygen was about three times that for pure hydrogen.

The interconversion of ortho and parahydrogen in the absence of molecular dissociation is the result of an intra-molecular rearrangement of pro-

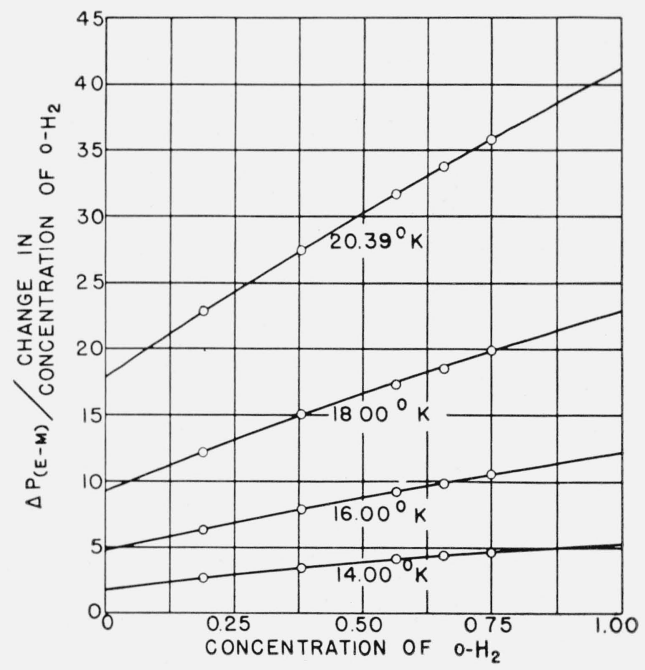

Figure 20. Deviations of vapor pressure of ortho-para $\mathrm{H}_{2}$ mixtures from law of ideal solutions. 
tons in the presence of a strong magnetic field, inhomogeneous on a scale of molecular dimensions.

As $p-\mathrm{H}_{2}$ has no net nuclear magnetic moment, the self conversion of nonequilibrium mixtures results only from the interaction of $0-\mathrm{H}_{2}$ molecules, which do have a nuclear magnetic moment, with each other and with $p-\mathrm{H}_{2}$ molecules. Hence, the ortho-para conversion in liquid and solid $\mathrm{H}_{2}$ is a bimolecular change.

$$
-d\left[o-H_{2}\right] / d t=k_{1}\left[o-\mathrm{H}_{2}\right]^{2}-k_{2}\left[o-\mathrm{H}_{2}\right]\left[p-\mathrm{H}_{2}\right]
$$

The velocity constant $k_{2}$ is much smaller than $k_{1}$ in accord with the small equilibrium proportion of $o-H_{2}$. At equilibrium, where $d\left[0-\mathrm{H}_{2}\right] / d t$ is zero, $k_{2} / k_{1}=\left[0-\mathrm{H}_{2}\right] /\left[p-\mathrm{H}_{2}\right]$. Values of equilibrium concentrations are given in table 12. For liquid hydrogen the velocity constant $k_{1}$ for conversion is 0.0114 per hour when concentrations are expressed in mole fractions. The value of $k_{1}$ for solid $\mathrm{H}_{2}$, $0.019 \mathrm{hr}^{-1}$ [147], is larger than for liquid $\mathrm{H}_{2}$ but decreases with time due to the immobility of molecules in the solid. The initial value of $k_{1}$ is restored however by melting and freezing.

\section{(b) $\mathrm{D}_{2}$}

The vapor pressures of normal and equilibrium deuterium were measured [149] relative to the vapor pressure of liquid $n-\mathrm{H}_{2}$ from $14^{\circ}$ to $20.4^{\circ} \mathrm{K}$. As these measurements are independent of a temperature scale their functional relations are given. Vapor pressures are expressed in terms of $\mathrm{mm}$ of $\mathrm{Hg}$ at standard conditions.

Normal deuterium (66.67 percent $0-\mathrm{D}_{2}, 33.33$ percent $\left.p-\mathrm{D}_{2}\right)$ :

Liquid: $\log _{10} P\left(n-\mathrm{D}_{2}\right)=-1.3376+1.3004 \log _{10}$

$$
P\left(n-\mathrm{H}_{2}\right) \text {. }
$$

Solid: $\log _{10} P\left(n-\mathrm{D}_{2}\right)=-1.9044+1.5143$

$$
\log _{10} P\left(n-\mathrm{H}_{2}\right) \text {. }
$$

20.4K Equilibrium deuterium (97.8 percent $o-\mathrm{D}_{2}, 2.2$ percent $p-\mathrm{D}_{2}$ ):

Liquid: $\log _{10} P\left(e-\mathrm{D}_{2}\right)=-1.3302+1.3000$

$$
\log _{10} P\left(n-\mathrm{H}_{2}\right) \text {. }
$$

Solid: $\log _{10} P\left(e-\mathrm{D}_{2}\right)=-1.8873+1.5106$

$$
\log _{10} P\left(n-\mathrm{H}_{2}\right) \text {. }
$$

Substituting for $\log _{10} P\left(n-\mathrm{H}_{2}\right)$ values given by eq. 7.2 for liquid $n-\mathrm{H}_{2}$ the following equations for $\log _{10} P\left(\mathrm{D}_{2}\right)$ are obtained:
Normal deuterium (66.67 percent $0-\mathrm{D}_{2}, 33.33$ percent $p-\mathrm{D}_{2}$ ):

Liquid: $\log _{10} P(\mathrm{~mm} \mathrm{Hg})=4.7312-\frac{58.4619}{T}$

$$
+0.02671 T \text {. }
$$

Solid: $\log _{10} P(\mathrm{~mm} \mathrm{Hg})=5.1626-\frac{68.0782}{T}$

$$
+0.03110 T \text {. }
$$

$20.4^{\circ} \mathrm{K}$ equilibrium deuterium (97.8 percent $o-\mathrm{D}_{2}, 2.2$ percent $p-\mathrm{D}_{2}$ )

Liquid: $\log _{10} P(\mathrm{~mm} \mathrm{Hg})=4.7367-\frac{58.4440}{T}$

$$
+0.02670 T \text {. }
$$

$$
\text { Solid: } \log _{10} P(\mathrm{~mm} \mathrm{Hg})=5.1625-\frac{67.9119}{T}
$$

$$
+0.03102 T \text {. }
$$

The triple-point temperatures and pressures for $\mathrm{D}_{2}$ given in tables 28 and 29 were obtained by simultaneous solution of the vapor pressure equations for solid and liquid.

The self conversion of nonequilibrium mixtures of $o$ - and $p-\mathrm{D}_{2}$ proceeds at a very much slower rate than for $\mathrm{H}_{2}$. Thus no increase in the vapor pressure of liquid $n-\mathrm{D}_{2}$ resulting from self conversion was observed at $20.4^{\circ} \mathrm{K}$ over a period of 100 hours [149]. The estimated probable error of two observations extending over 100 -hour periods was $\pm 0.27 \mathrm{~mm} \mathrm{Hg}$. The small rate of self conversion of $\mathrm{D}_{2}$, compared with $\mathrm{H}_{2}$, is a result of the smaller magnetic moment of the deuteron compared with the proton. The ratio of nuclear magnetic moments $\mathrm{D} / \mathrm{H}$ is 0.26 . The relative rate of self conversion for the same displacements of $\mathrm{D}_{2}$ and $\mathrm{H}_{2}$ from the equilibrium ortho-para composition is proportional, as to order of magnitude only, to the fourth power of their relative magnetic moments, that is to 0.005. Allowing for the smaller displacement of $n-\mathrm{D}_{2}$ from equilibrium composition and the smaller difference between the vapor pressures of the ortho and para varieties of $D_{2}$, the expected ratio of the rates of vapor pressure change, $n-\mathrm{D}_{2}$ to $n-\mathrm{H}_{2}$, is of the order of $10^{-3}$. For a more detailed discussion see reference [149].

$$
\text { (c) HD }
$$

As the two nuclei of the HD molecule are dissimilar, hydrogen deuteride does not have ortho and para varieties. Measurements of the vapor 
pressure of $\mathrm{HD}$ extend from $10.4^{\circ}$ to $20.4^{\circ} \mathrm{K}$ [150]. The following vapor-pressure equations were made to fit the triple-point temperature $16.60_{4}{ }^{\circ} \mathrm{K}$ measured with a platinum resistance thermometer in a calorimeter in which the solid and liquid phases were in equilibrium.

HD:

Liquid: $\log _{10} P(\mathrm{~mm} \mathrm{Hg})=5.04964-$

$$
\frac{55.2495}{T}+0.01479 T
$$

Solid: $\log _{10} P(\mathrm{~mm} \mathrm{Hg})=4.70260$

$$
\frac{56.7154}{T}+0.04101 T
$$

The triple-point pressure of HD given in tables 28 and 29 can be obtained from either of these equations.

\section{(d) HT and DT}

Tritium, $\mathrm{T}$, the hydrogen isotope of atomic weight 3 is radioactive and has a half-lifetime of $31 \pm 8$ years [151]. Its disintegration products are a negative $\beta$-particle and $\mathrm{He}^{3}$. Because of its comparatively short half-life, the natural abundance of $\mathrm{T}$ in hydrogen is extremely small. Libby and Barter [152] determined the vapor pressures of HT and DT using T made by the irradiation of a block of metallic Li with neutrons $\left(\mathrm{Li}^{6}+\mathrm{n} \rightarrow\right.$ $\left.\mathrm{He}^{4}+\mathrm{T}^{3}\right)$. The tritium held by the Li as LiT was liberated by the reaction of $\mathrm{H}_{2} \mathrm{O}$ or $\mathrm{D}_{2} \mathrm{O}$ with the Li block. Gaseous $\mathrm{H}_{2}$ or $\mathrm{D}_{2}$ with a trace of $\mathrm{HT}$ or D'T was obtained. The gas was liquefied and then evaporated, and the radioactivity of the evaporated vapor was measured as a function of the volume of the remaining unevaporated liquid. From a comparison of the radioactivity of the vapor leaving the liquid during different periods of the evaporation, Libby and Barter calculated the vapor pressures of H'T and DT, making use of ideal solution laws for this purpose. They obtained for the vapor pressures of HT and $\mathrm{DT}$, $254 \pm 16$ and $123 \pm 6 \mathrm{~mm} \mathrm{Hg}$, respectively, at the normal boiling temperature of hydrogen $\left(20.39^{\circ} \mathrm{K}\right)$. By extrapolation, they estimated that the vapor pressure of $\mathrm{T}_{2}$ at $20.39^{\circ} \mathrm{K}$ is $45 \pm 10 \mathrm{~mm} \mathrm{Hg}$.

\section{Pressure-Temperature Relations for Solid-Liquid Equilibrium}

The melting, or freezing pressures, of $n-\mathrm{H}_{2}$, $\mathrm{HD}$, and $n-\mathrm{D}_{2}$ given in table 30 are based on smooth curves drawn through the experimental data $\left(\mathrm{H}_{2},[153\right.$ to 157$] ; \mathrm{HD}$ [150]; $\mathrm{D}_{2}$ [174]) and cover the same ranges of pressure and temperature as the data. Figure 21 is a diagram of the deviations of the data for $n-\mathrm{H}_{2}$ from the table. The dashed line shows a 1-percent deviation from the table and the full-line curve represents the deviation from the table of the equation

$\log _{10}(237.1+P)=1.85904 \log _{10} T+0.24731, \quad(7.18)$, where $P$ is in $\mathrm{kg} \mathrm{cm}^{-2}$.

TABLE 30. Melting temperature-pressure relations for

\begin{tabular}{|c|c|c|c|}
\hline \multirow{2}{*}{$\mathrm{T}$} & \multicolumn{3}{|c|}{$\Gamma$} \\
\hline & $n-\mathrm{H}_{2}$ & HD & $n-\mathrm{D}_{2}$ \\
\hline${ }^{\circ} K$ & $\mathrm{~kg} \mathrm{~cm}-2$ & $\mathrm{~kg} \mathrm{cm-2}$ & $\mathrm{kg} \mathrm{cm}^{-2}$ \\
\hline 13.96 & 0.07 & - & - \\
\hline 14 & 1.4 & - & - \\
\hline $15 \ldots$ & 33.2 & - & - n..... \\
\hline 16 & 67.3 & - n & - n..... \\
\hline $16.60 \ldots$ & - & 0.13 & - \\
\hline $17 \ldots$ & 103.5 & 14.2 & - \\
\hline $18 \ldots$ & 142.3 & 52.6 & - \\
\hline $18.72 \ldots$ & - & - & 0.17 \\
\hline 19 & 183.6 & 92.9 & 13.9 \\
\hline $20 \ldots$ & 227.1 & - & 56. 0 \\
\hline $21 \ldots \ldots$ & 272.3 & - & 100. 0 \\
\hline 22 & 318.6 & (-non & - \\
\hline 23 & 366.0 & - & - \\
\hline 24 & 415.0 & - & - \\
\hline $25 \ldots$ & 465.6 & - & - \\
\hline $26 \ldots \ldots$ & 518 & - & - \\
\hline $27 \ldots \ldots \ldots-1$ & 572 & (n) & -...... \\
\hline 28 & 628 & - & (........ \\
\hline 29 & 685 & - & (n...... \\
\hline 30 & 744 & - & - \\
\hline 32 & 867 & - & - \\
\hline 34 & 996 & - & - \\
\hline 36 & 1,131 & - & - \\
\hline 38 & 1,274 & - & (n...... \\
\hline 40 & 1,422 & (n) & - \\
\hline 45 & 1,821 & - & - \\
\hline 50 & 2,258 & (-...... & - \\
\hline 55 & 2,735 & - & - \\
\hline 60 & 3,249 & (n) & - \\
\hline 65 & 3,801 & -...- & - \\
\hline $70 \ldots$ & 4,389 & - & - \\
\hline 75 & 5,014 & - & - \\
\hline $80 \ldots$ & 5,674 & (n) & - \\
\hline
\end{tabular}
$n-\mathrm{H}_{2}, \mathrm{HD}$, and $n-\mathrm{D}_{2}$

Figure 22 is intended to show the relation between the melting pressures of $n-\mathrm{H}_{2}, \mathrm{HD}$, and $n-\mathrm{D}_{2}$. The curve for $n-\mathrm{H}_{2}$ is a graph of table values. The curves through the experimental 


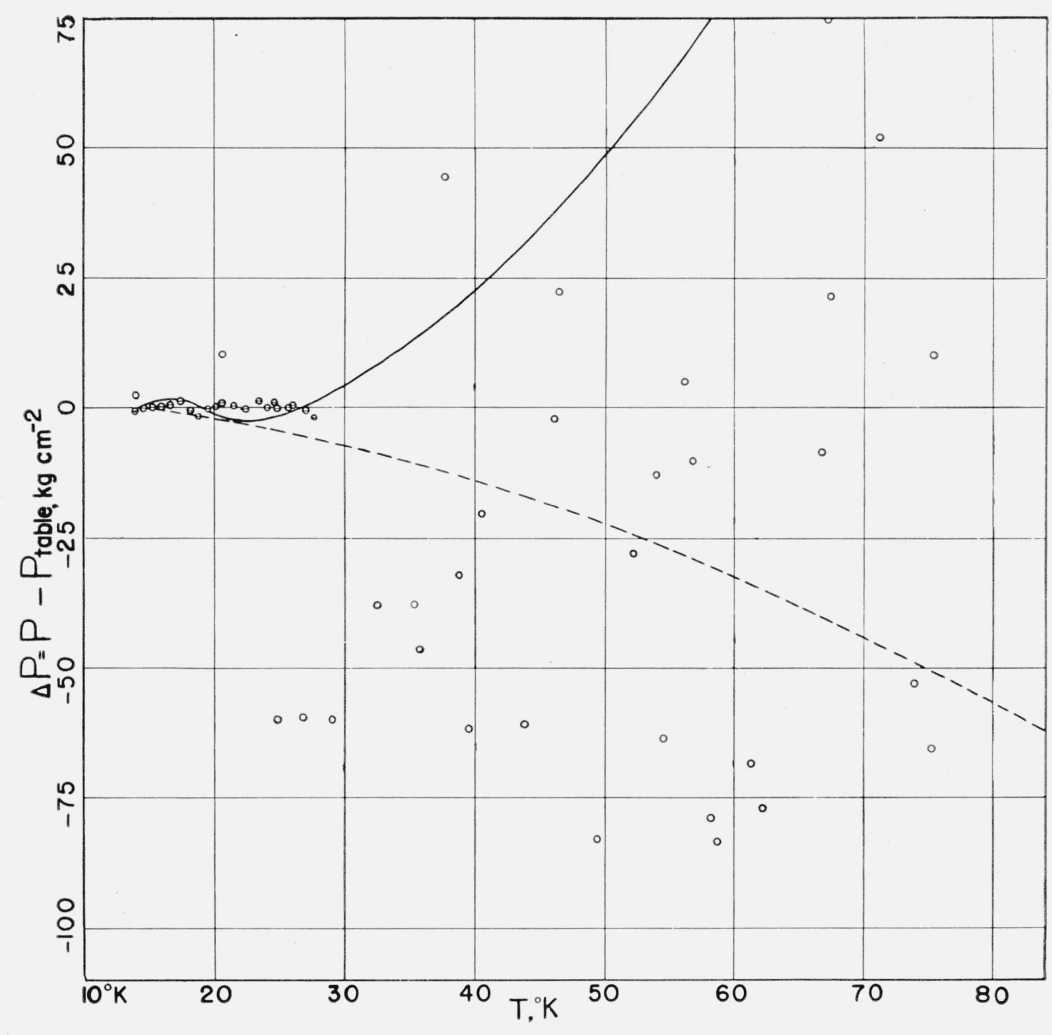

Figure 21. Melting pressure of $n-\mathrm{H}_{2}$ as a function of temperature.

data for $\mathrm{HD}$ and $\mathrm{D}_{2}$ were obtained by a simple vertical displacement of the $\mathrm{H}_{2}$ curve and show that the differences in melting pressures of the three isotopic varieties are only slightly dependent upon the temperature. These differences in pressure are $89.6 \mathrm{~kg} \mathrm{~cm}^{-2}$ for $\mathrm{H}_{2}$ and $\mathrm{HD}$ and $170.6 \mathrm{~kg} \mathrm{~cm}-2$ for $\mathrm{H}_{2}$ and $\mathrm{D}_{2}$. As the change of melting pressure with temperature, $d P / d T$, has nearly the same value for $\mathrm{H}_{2}, \mathrm{HD}$, and $\mathrm{D}_{2}$, if compared at the same temperature, it follows from the Clapeyron equations that $L_{f} / \delta V$, the ratio of the heat of fusion to the change in volume on melting, also has nearly the same value for the three isotopes when compared at the same value of T. A similar statement can be made for $S_{f} / \delta V$, the ratio of the entropy of fusion to the change in volume on melting.

The table values of melting pressure for $\mathrm{HD}$ and $\mathrm{D}_{2}$ were obtained from curves drawn through the experimental data and not from the curves of figure 22 .

\section{PVT Data for the Condensed States}

The available date of state for the condensed phases of $\mathrm{H}_{2}, \mathrm{HD}$, and $\mathrm{D}_{2}$ are meager [ 158 to 166 ] and in general not accurate enough for the calculation of reliable values of thermodynamical properties. The data on the liquid, however, were used in the construction of the liquid regions of the $\sigma$ versus $\rho$ diagrams, figure 6 , and the $T$ versus $S$ diagram, figures 31,32 , and 33 .

\section{Liquid $\mathrm{H}_{2}, \mathrm{HD}$, and $\mathrm{D}_{2}$}

In table 31 are given the molar volumes of liquid $n-\mathrm{H}_{2}, p-\mathrm{H}_{2}, \mathrm{HD}$, and $n-\mathrm{D}_{2}$ in equilibrium 


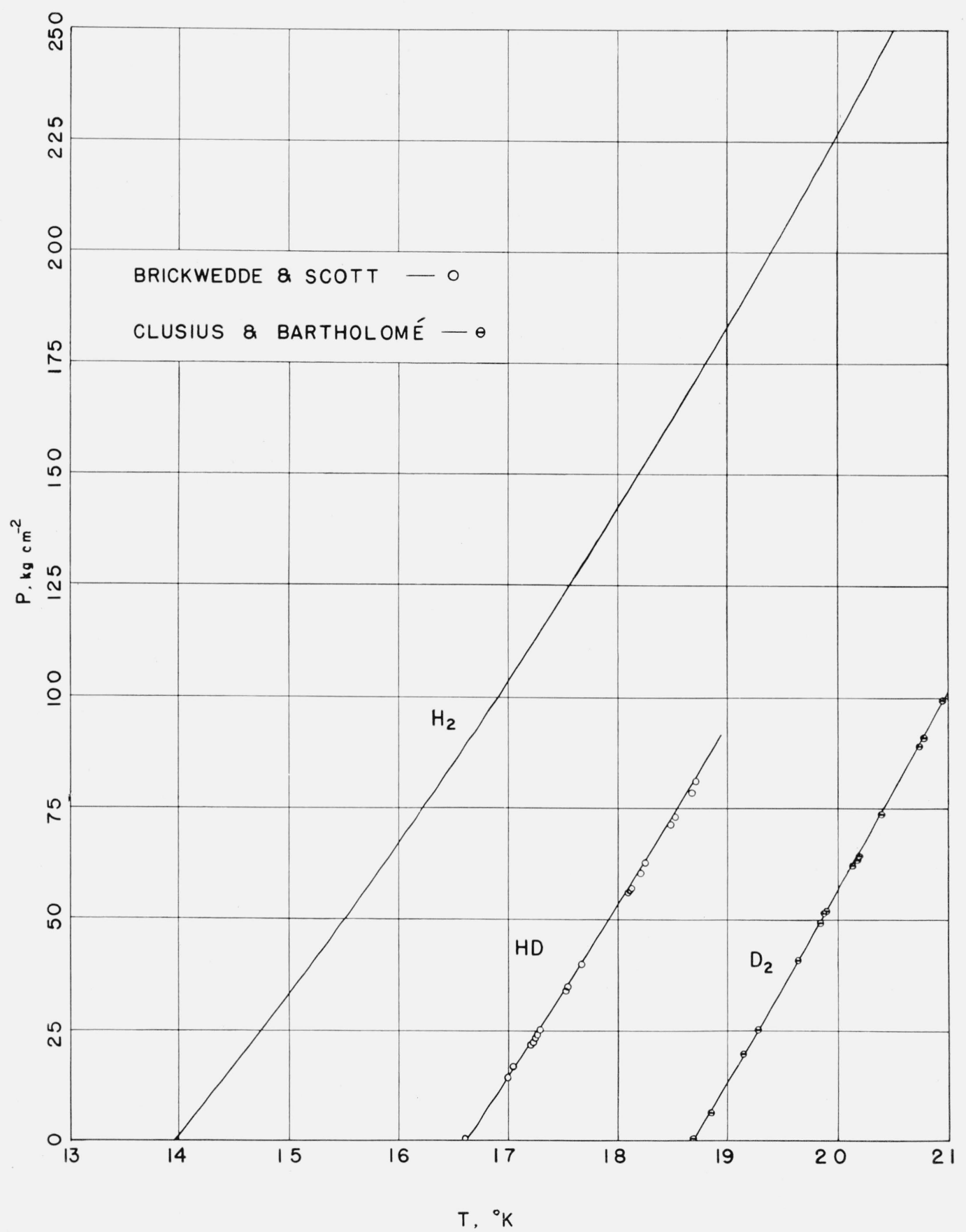

Figure 22. Melting pressures of $n-\mathrm{H}_{2}, \mathrm{HD}$, and $n-\mathrm{D}_{2}$. 
TABLE 31. Molar volumes of normal hydrogen, parahydrogen, normal deuterium, and hydrogen deuteride, in the liquid state

\begin{tabular}{|c|c|c|c|c|}
\hline \multirow{2}{*}{$T$} & \multicolumn{4}{|c|}{ Volume of liquid at saturation pressure } \\
\hline & $n-\mathrm{H}_{2}$ & $p-\mathrm{H}_{2}$ & $n-\mathrm{D}_{2}$ & HD \\
\hline${ }^{\circ} K$ & $\mathrm{~cm}^{3} \mathrm{~mole}^{-1}$ & $c m^{3} m^{2} e^{-1}$ & $c m^{3} m_{o l e}-1$ & $\mathrm{~cm}^{3} \mathrm{~mole}^{-1}$ \\
\hline $13.813 \ldots$ & -....... & 26. 176 & ........ & -....... \\
\hline 13. $96 \ldots \ldots$ & 26. 108 & - n..... & -....... & - n..... \\
\hline 14 & 26. 119 & 26.227 & - n-...- & (n) \\
\hline 15 & 26.407 & 26.518 & -.....-. & - . - n... \\
\hline 16 & 26. 721 & 26. 836 & - n-.... & - nne \\
\hline 16.604 & -....... & - n..... & - n...... & 24.487 \\
\hline 17 & 27.061 & 27.179 & - n. & 24.594 \\
\hline 18 & 27.426 & 27.549 & -........ & 24.885 \\
\hline 18.723 & - n...... & - & 23.162 & - \\
\hline 19 & 27.816 & 27.945 & 23. 237 & 25.211 \\
\hline $20 \ldots \ldots$ & 28. 232 & 28.368 & 23.525 & 25.572 \\
\hline 20.39 & 28.401 & - n & - n. & - n \\
\hline 22 & 29.233 & - & - n.... & - n..... \\
\hline 24 & 30.451 & - n & - & - \\
\hline 26 & 31.995 & -........ & -....... & -........ \\
\hline $28 \ldots \ldots$ & 34.059 & - & - n. & ....... \\
\hline 30 & 37.138 & - & - & - \\
\hline 32 & 43. 211 & - n & - & - \\
\hline 33.19 & 66.95 & - n & n...... & - \\
\hline
\end{tabular}

with vapor from the triple point to the highest temperature of measurement. From the triple point to $20.4^{\circ} \mathrm{K}$, these equilibrium molar volumes have been represented by the following equations, in which temperatures are on the Kelvin scale:

Normal hydrogen [163]:

$V\left(\mathrm{~cm}^{3} \mathrm{~mole}^{-1}\right)=24.747-0.08005 T+0.012716 T^{2}$.

Parahydrogen [163]:

$V\left(\mathrm{~cm}^{3} \mathrm{~mole}^{-1}\right)=24.902-0.0888 T+0.013104 T^{2}$.

Hydrogen deuteride [150]:

$V\left(\mathrm{~cm}^{3} \mathrm{~mole}^{-1}\right)=24.886-0.30911 T+0.01717 T^{2}$.

Normal deuterium [174]:

$V\left(\mathrm{~cm}^{3} \mathrm{~mole}^{-1}\right)=22.965-0.2460 T+0.0137 T^{2}$.

Table values at $20.39^{\circ} \mathrm{K}$ and lower were calculated from these equations. Values of the molar volume of liquid normal hydrogen above $20.4^{\circ} \mathrm{K}$ were obtained from the experimental data of Mathias, Crommelin, and Onnes [161] with the help of a sensitive interpolation method based upon the use of an empirical equation and a deviation graph. A change was made in the experimental data because the value used by Mathias, Crommelin, and Onnes for the density of gaseous hydrogen at standard conditions differs from that recommended in this paper on page 396 .

Bartbolomé [177] measured the molar volumes of liquid $n-\mathrm{H}_{2}$ and $n-\mathrm{D}_{2}$ as a function of pressure at three temperatures between $16^{\circ}$ and $21^{\circ}$ $\mathrm{K}$. The measurements extended from the vapor pressure to nearly the freezing pressure. Smoothed values of molar volumes are given in tables 32 and 33. Bartholomé showed that isothermal changes in volume to about 9 percent of the volume of "saturated" liquid can be represented to within the precision of his measurements, $\pm 0.05 \mathrm{~cm}^{3}$ mole $e^{-1}$ by Eucken's equation

$$
\frac{1}{V^{3}}=\frac{1}{2}\left[\frac{1}{v_{0}^{3}}+\sqrt{\frac{1}{v_{0}^{6}}+a P}\right],
$$

in which $\mathrm{V}$, the molar volume of the liquid, is expressed as a function of the pressure $P . v_{0}$ is the molar volume extrapolated to zero pressure, and $a$ is an empirical constant dependent upon the temperature. Tables 32 and 33 include values of the molar volumes of liquid $n-\mathrm{H}_{2}$ and $n-\mathrm{D}_{2}$ at freezing pressure for the three temperatures of Bartholomé's measurements.

TABLE 32. Molar volumes of liquid $n-\mathrm{H}_{2}$ for various temperatures and pressures.

\begin{tabular}{|c|c|c|c|}
\hline Pressure & $T=16.43^{\circ} \mathrm{K}$ & $T=18.24^{\circ} \mathrm{K}$ & $T=20.33^{\circ} \mathrm{K}$ \\
\hline $\mathrm{kg} \mathrm{cm-2}$ & $\mathrm{cm}^{3} \mathrm{~mole}^{-1}$ & $\mathrm{~cm}^{3} \mathrm{~mole}^{-1}$ & $c m^{3}$ mole $^{-1}$ \\
\hline $0^{a} \ldots \ldots$ & 26.87 & 27.54 & 28.43 \\
\hline $10 \ldots \ldots$ & 26.59 & 27.18 & 27.97 \\
\hline $25 \ldots \ldots$ & 26. 20 & 26.72 & 27.40 \\
\hline $50 \ldots \ldots$ & 25.66 & 26.10 & 26.62 \\
\hline $75 \ldots \ldots$ & 25.20 & 25.59 & 25.98 \\
\hline $82.6 \ldots$ & 25.08 & & \\
\hline $100 \ldots$ & - & 25.14 & 25. 42 \\
\hline $125 \ldots \ldots$ & - & 24.71 & 24.91 \\
\hline $150 \ldots$ & & 24.30 & 24.47 \\
\hline $151.98 \ldots$ & - & 24.27 & -...... \\
\hline $175_{\ldots} \ldots$ & & & 24.09 \\
\hline $200 \ldots$ & $\ldots$ & - & 23.76 \\
\hline $225 \ldots \ldots$ & - & $-\ldots-$ & 23.48 \\
\hline $241.83 \ldots$ & & - & 23.31 \\
\hline & $a=3.80 \times 10^{-11} \frac{\mathrm{mole}^{6}}{\mathrm{~cm}^{16} \mathrm{~kg}}$ & $a=3.93 \times 10^{-11} \frac{\mathrm{mole}^{6}}{\mathrm{~cm}^{16} \mathrm{~kg}}$ & $a=4.16 \times 10^{-11} \frac{\mathrm{mole}^{6}}{\mathrm{~cm}^{16} \mathrm{~kg}}$ \\
\hline
\end{tabular}

$a$ The values at zero pressure were obtained by extrapolation consistent with the molar volumes at saturation vapor pressure given by eq. 8.1. 
TABLE 33. Molar volumes of liquid $n$ - $\mathrm{D}_{2}$ for various temperatures and pressures

\begin{tabular}{|c|c|c|c|}
\hline Pressure & $T=19.70^{\circ} \mathrm{K}$ & $T=20.31^{\circ} \mathrm{K}$ & $T=20.97^{\circ} \mathrm{K}$ \\
\hline $\mathrm{kg} \mathrm{cm}^{-2}$ & $c m^{3}$ mole $e^{-1}$ & $\mathrm{~cm}^{3} \mathrm{~mole}^{-1}$ & $\mathrm{~cm}^{3}$ mole $^{-1}$ \\
\hline $0^{a} \ldots$ & 23.44 & 23.63 & 23.84 \\
\hline $10 \ldots \ldots$ & 23. 24 & 23.37 & 23.59 \\
\hline $20 \ldots \ldots$ & 23. 06 & 23.16 & 23.35 \\
\hline $30 \ldots \ldots$ & 22.89 & 22.97 & 23. 14 \\
\hline $40 \ldots \ldots$ & 22.74 & 22.79 & 22.95 \\
\hline 43.18 & 22. 70 & & \\
\hline $50 \ldots \ldots$ & - & 22.63 & 22. 77 \\
\hline $60 \ldots \ldots$ & .... & 22.49 & 22. 60 \\
\hline $69.46 \ldots$ & & 22.36 & \\
\hline $70 \ldots$ & & & 22.45 \\
\hline $80 \ldots$ & & & 22.30 \\
\hline \multirow{3}{*}{$90 \ldots}$. & & 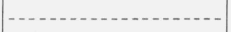 & 22.16 \\
\hline & & - & 22.05 \\
\hline & $a=6.75 \times 10^{-11} \frac{\mathrm{mole}}{\mathrm{cm}^{16} \mathrm{~kg}}$ & $a=7.20 \times 10^{-11} \frac{\mathrm{mole}}{\mathrm{cm}^{16} \mathrm{~kg}}$ & $a=7.37 \times 10^{-11} \frac{\mathrm{mole} 6}{\mathrm{~cm}^{16} \mathrm{~kg}}$ \\
\hline
\end{tabular}

$a$ The values at zero pressure were obtained by extrapolation consistent with the molar volumes at saturation vapor pressure given by eq 8.4.

\section{Solid $\mathrm{H}_{2}, \mathrm{HD}$, and $\mathrm{D}_{2}$}

The crystal structure of solid hydrogen is thought to be hexagonal close-packed, on the basis of an X-ray investigation of solid parahydrogen by the Debye-Scherrer method at the temperature of liquid helium, conducted by Keesom, de Smedt, and Mooy [162].

Tables 34 and 35 contain all the available experi- mental data of state on solid $\mathrm{H}_{2}, \mathrm{HD}$, and $\mathrm{D}_{2}$. Molar volumes at $0^{\circ} \mathrm{K}$ were obtained by calculation.

Molar volumes of the solid at the triple point given in table 34 were obtained by subtracting the volume changes on fusion from the triple point volumes of the liquid calculated from eq 8.1, 8.3, and 8.4. The volume changes on fusion, given in table 34, were calculated using the Clapeyron equation with the calorimetrically measured heats of fusion (section IX, 3), and $d P / d T$ for the solidliquid equilibrium at the triple point (section VII, 2).

Molar volumes of the solid in table 34 above the triple-point temperature were obtained from Bartholome's measurements of the change in volume on fusion at the temperatures given in table 34, and the volumes of the liquid at melting pressure given in tables 32 and 33 .

The molar volumes of solid $\mathrm{H}_{2}$ and $\mathrm{D}_{2}$ at $4.2^{\circ}$ $\mathrm{K}$ in table 34 were measured by Megaw [165] with a picnometer in which the solid $\mathrm{H}_{2}$ or $\mathrm{D}_{2}$ was surrounded with liquid helium, the volume of which had previously been measured as a function of pressure at this temperature. The compressibilities of solid $\mathrm{H}_{2}$ and $\mathrm{D}_{2}$ at $4.2^{\circ} \mathrm{K}$, given in table 35, were calculated by Miss Megaw from the results of these measurements.

TABLE 34. Molar volumes of solid $n-\mathrm{H}_{2}, \mathrm{HD}$ and $n-\mathrm{D}_{2}$ and volume changes upon fusion

\begin{tabular}{|c|c|c|c|c|c|c|c|c|}
\hline \multirow[b]{2}{*}{$T$} & \multirow[b]{2}{*}{$P$} & \multicolumn{2}{|c|}{$n-\mathrm{H}_{2}$} & \multicolumn{2}{|c|}{$\mathrm{HD}$} & \multicolumn{2}{|c|}{$n-\mathrm{D}_{2}$} & \multirow[b]{2}{*}{ Remarks } \\
\hline & & $\begin{array}{l}\text { Volume } \\
\text { of solid }\end{array}$ & $\begin{array}{l}\text { Volume } \\
\text { change on } \\
\text { fusion }\end{array}$ & $\begin{array}{l}\text { Volume } \\
\text { of solid }\end{array}$ & $\begin{array}{l}\text { Volume } \\
\text { change on } \\
\text { fusion }\end{array}$ & $\begin{array}{l}\text { Volume } \\
\text { of solid }\end{array}$ & $\begin{array}{l}\text { Volume } \\
\text { change on } \\
\text { fusion }\end{array}$ & \\
\hline${ }^{\circ} K$ & $\mathrm{~kg} / \mathrm{cm}^{2}$ & $\mathrm{~cm}^{3} /$ mole & $\mathrm{cm}^{3} / \mathrm{mole}$ & $\mathrm{cm}^{3} /$ mole & $\mathrm{cm}^{3} /$ mole & $\mathrm{cm}^{3} /$ mole & $\mathrm{cm}^{3} /$ mole & \\
\hline $20.97 \ldots$ & 98.7 & & & & & 20.07 & 1.98 & \\
\hline $20.31 \ldots$ & 69.5 & & & & & 20.20 & 2.16 & $T$ and $P$ for solid-liquid equilibrium. \\
\hline 18.72 & $0.17_{4}$ & & & & & 20.48 & 2. 66 & $n-\mathrm{D}_{2}$ triple point. \\
\hline 16.60 & .126 & & & 21.84 & 2.65 & & & $\mathrm{HD}$ triple point. \\
\hline $18.24 \ldots \ldots$ & 152.0 & 22.24 & 2. 03 & & & & & Tond $P$ for golialiouid couilibrium \\
\hline $16.43 \ldots \ldots$ & 82.6 & 22.78 & 2. 30 & & & & & $T$ and $P$ for solid-liquid equilibrium. \\
\hline $13.96 \ldots$ & 0.073 & 23. 25 & 2. 85 & & & & & $n-\mathrm{H}_{2}$ triple point. \\
\hline $4.2 \ldots \ldots$ & 50 & 22.03 & & & & 19. 28 & & determination. \\
\hline $4.2 \ldots \ldots$. & 75 & 21.80 & & & & 19.16 & & \\
\hline 4.2 & 100 & 21.60 & & & & 19. 06 & & \\
\hline $0 \ldots \ldots$ & 0 & 22.57 & & & & 19. 49 & & By calculation. \\
\hline
\end{tabular}


TABLE 35. Experimentally determined compressibilities, $\frac{1}{V}\left(\frac{d V}{d P}\right)_{T}$, of solid $\mathrm{H}_{2}$ and $\mathrm{D}_{2}$ at $4.2^{\circ} \mathrm{K}$

\begin{tabular}{|c|c|c|}
\hline Compressibility & $\begin{array}{c}\mathrm{H}_{2} \text { compressi- } \\
\text { bility }\end{array}$ & $\begin{array}{c}\mathrm{D}_{2} \text { compressi- } \\
\text { bility }\end{array}$ \\
\hline $\begin{array}{l}\text { At pressure } 0 \mathrm{~kg} \mathrm{~cm}^{-2} \\
\text { At pressure } 100 \mathrm{~kg} \mathrm{~cm}^{-2} \\
\text { A verage for range } 0 \text { to } 100 \mathrm{~kg} \mathrm{~cm}^{-2}\end{array}$ & $\begin{array}{c}\mathrm{kg}^{-1} \mathrm{~cm}^{2} \\
(6.8 \pm 1.5) \times 10^{-4} \\
3.2 \times 10^{-4} \\
(5.0 \pm 0.5) \times 10^{-4}\end{array}$ & $\begin{array}{c}k g^{-1} \mathrm{~cm}^{2} \\
(4.5 \pm 2) \times 10^{-4} \\
2.1 \times 10^{-4} \\
(3.3 \pm 0.7) \times 10^{-4}\end{array}$ \\
\hline
\end{tabular}

Miss Megaw calculated the expansivities of solid $\mathrm{H}_{2}$ and $\mathrm{D}_{2}$ at $4.2^{\circ}$ and $11^{\circ} \mathrm{K}$, given in table 36 , using the formula

$$
C_{p}-C_{v}=T V\left(\frac{d V}{d T}\right)_{P}^{2} /\left(\frac{d V}{d P}\right)_{T}
$$

with the calorimetrically determined specific heats at constant pressure and volume, and the compressibility measured at $4.2^{\circ} \mathrm{K}$ (table 35 ).

TABLE 36. Expansivities, $\frac{1}{V}\left(\frac{d V}{d T}\right)_{P}$, of solid $\mathrm{H}_{2}$ and $\mathrm{D}_{2}$ calculated from $C_{p}-C_{v}$

\begin{tabular}{|c|c|c|}
\hline$T$ & $\mathrm{H}_{2}$ & $\mathrm{D}_{2}$ \\
\cline { 1 - 3 }${ }^{\circ} K$ & ${ }^{\circ} K^{-1}$ & ${ }^{\circ} K^{-1}$ \\
4.2 & $0.24 \times 10^{-2}$ & $0.17 \times 10^{-2}$ \\
11 & $.51 \times 10^{-2}$ & $.37 \times 10^{-2}$ \\
\hline
\end{tabular}

The compressibilities and expansivities of solid $\mathrm{H}_{2}$ and $\mathrm{D}_{2}$ are large when compared with values of these properties for other substances. This is ascribed to the zero-point vibrational energy of the lattice which for hydrogen is an unusually large fraction of the negative potential energy of the lattice. This accounts also for an unusually large variation in the compressibilities of $\mathrm{H}_{2}$ and $\mathrm{D}_{2}$ with pressure (see table 35), and for the variation with $T$ and $V$ of $d \ln \theta / d \ln V$, which derivative of the Debye $\theta$ is usually regarded as a constant for other solids [165].

\section{The Thermal Properties of the Condensed Phases}

In this section are included the calorimetrically measured properties: specific heats and heats of fusion and vaporization.

\section{Specific Heats of the Solids and Liquids}

\section{(a) Hydrogen}

The specific heats at saturation pressure of solid and liquid hydrogen were measured (1923) by
Simon and Lange [171] between $10^{\circ}$ and $20^{\circ} \mathrm{K}$, before the discovery of parahydrogen. Clusius and Hiller [172] measured (1929) the specific heats of solid and liquid parahydrogen over the same range of temperatures and obtained the same values, within experimental error, for the specific heats of parahydrogen as had been obtained by Simon and Lange for supposedly normal hydrogen. Mendelsohn, Ruhemann, and Simon [173] measured (1931) the specific heats of several mixtures of ortho- and parahydrogen between $2.5^{\circ}$ and $11.5^{\circ} \mathrm{K}$. Their results on pure parahydrogen were in agreement with the earlier measurements of Clusius and Hiller, the data from $2.5^{\circ}$ to

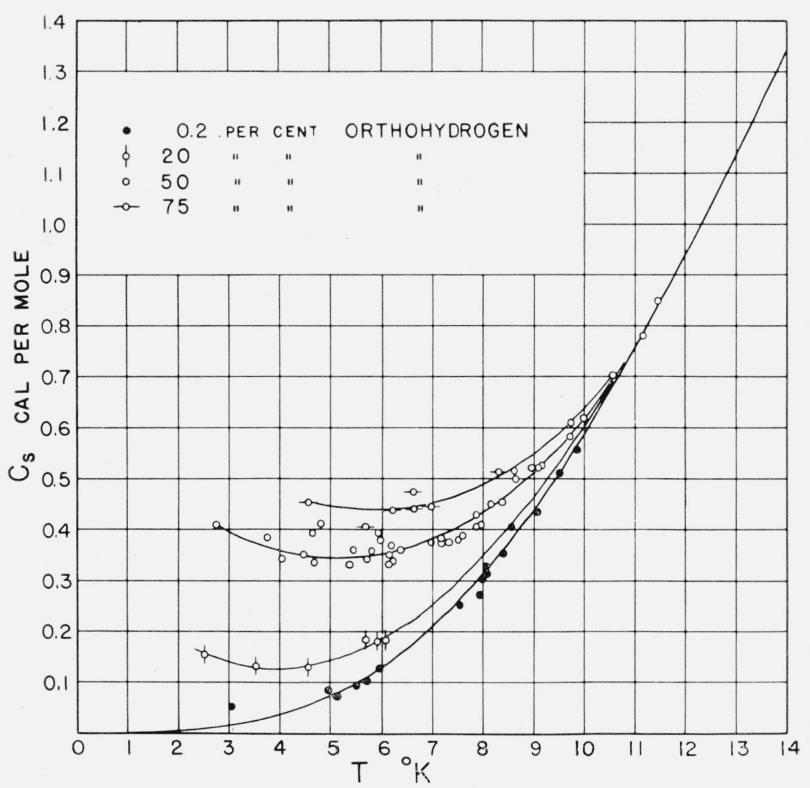

Figure 23. Specific heat, $C_{s}$, of solid $\mathrm{H}_{2}$ for various ortho-para compositions.

$14^{\circ} \mathrm{K}$ fitting rather closely a Debye function with $\theta=91^{\circ} \mathrm{K}$.

The data of Mendelsohn, Ruhemann, and Simon are shown in figure 23. It is seen that, at temperatures below $11^{\circ} \mathrm{K}$, the specific heats of mixtures containing orthohydrogen are larger than for pure parahydrogen. This difference in specific heats is connected with the multiplicity of states belonging to the lowest $0-\mathrm{H}_{2}$ rotational level, $J=1$. The different states, three in number, correspond to three different orientations of the angular momentum vector of an $0-\mathrm{H}_{2}$ molecule relative to the electric field in the hydrogen crystal. At $0^{\circ} \mathrm{K}$, all $0-\mathrm{H}_{2}$ molecules are in the orientation state of lowest energy. At tempera- 
tures of the order of $\Delta E / k$, where $\Delta E$ is the difference in the energy of the states, the distribution of $\mathrm{o}-\mathrm{H}_{2}$ molecules over the three states changes rapidly with change of temperature. Along with this there is an absorption of energy and an increase in specific heat. As temperatures are approached that are high compared with $\Delta E / k$, the distribution of $0-\mathrm{H}_{2}$ molecules becomes uniform over the three orientation states, and the specific heat of orientation approaches zero. It may be seen from figure 23 that $12^{\circ} \mathrm{K}$ is effectively a high temperature for this distribution, and that at temperatures above $12^{\circ} \mathrm{K}$ the distribution over the three $J=1$ states must be practically uniform.

The specific heats, $C_{s}$, of liquid and solid hydrogen along the saturated vapor lines are given in table 37 . The $C_{s}$ curves of figures 24 , 25 , and 26 for $n-\mathrm{H}_{2}$ at temperatures above $11^{\circ} \mathrm{K}$ represent this table.

In figures 25 and 26 the heat capacity, $C_{v}$, of

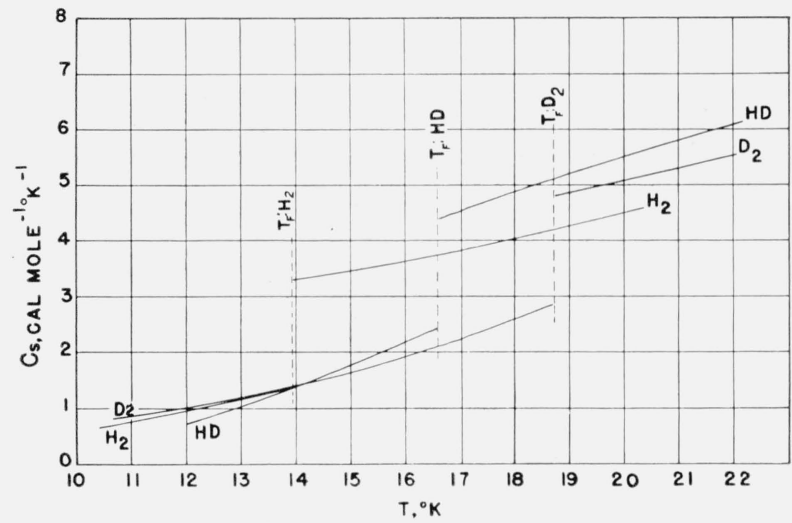

Figure 24. Specific heat, $C_{s}$, of solid and liquid $\mathrm{H}_{2}, \mathrm{HD}$, and $\mathrm{D}_{2}$.

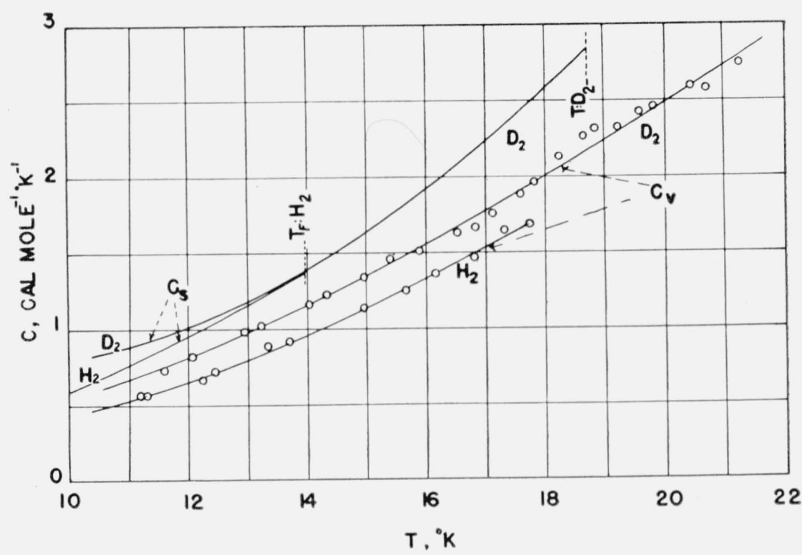

Figure 25. Specific heats, $C_{s}$ and $C_{v}$, of solid $\mathrm{H}_{2}$ and $\mathrm{D}_{2}$

solid and of liquid $n$ - $\mathrm{H}_{2}$ at constant specified values of the density are compared with the heat capacity, $C_{s}$, of solid and liquid $n-\mathrm{H}_{2}$ in equilibrium with saturated vapor. It is to be noted that the $C_{v}$ curves of these two figures are not for $C_{v}$ of solid and liquid $\mathrm{H}_{2}$ along a line of equilibrium of vapor and condensed phase. The $C_{v}$ measurements on the solid were made by Bartholomé and Eucken [176] at the density of solid $\mathrm{H}_{2}$ at a melting temperature of about $19^{\circ} \mathrm{K}$. The $C_{v}$ measurements for the liquid were made by Eucken [169] and by Bartholomé and Eucken at densities ranging from 0.034 to $0.077 \mathrm{~g} \mathrm{~cm}^{-3}$ (380 Amagats to 860 Amagats). The density of liquid $n-\mathrm{H}_{2}$ at its normal boiling point is $0.07097 \mathrm{~g} \mathrm{~cm}^{-3}$ (789.7 Amagats).

The difference between $C_{v}$ in figure 25 for the solid at constant density and $C_{v}$ at densities of the solid along the solid-vapor equilibrium line is small. The corresponding difference for the liquid is larger and, at the critical temperature $33.19^{\circ} \mathrm{K}$, is of the order of 1 or 2 cal mole ${ }^{-1}{ }^{\circ} \mathrm{K}^{-1}$

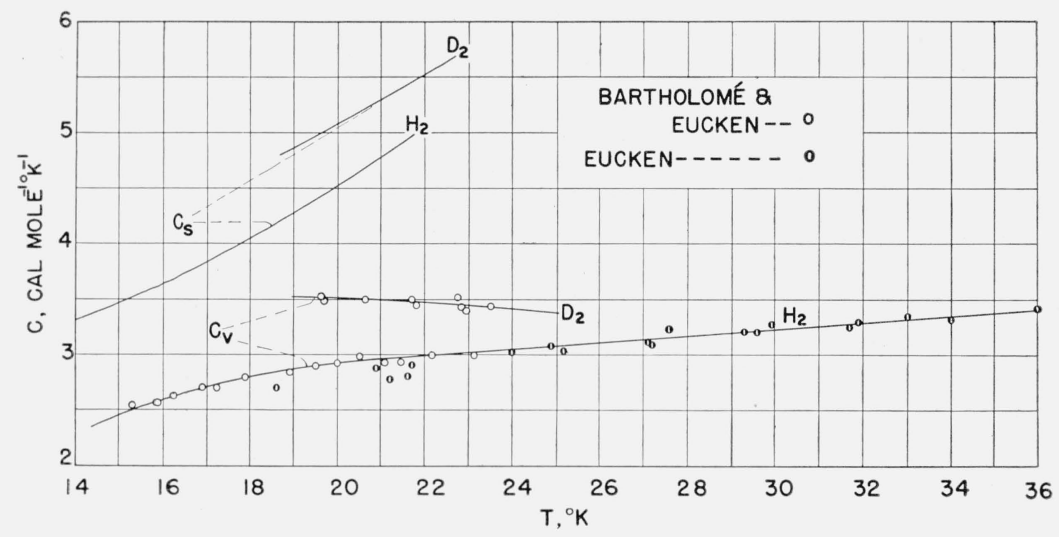

Figure 26. Specific heats, $C_{\mathbf{s}}$ and $C_{v}$, of liquid $\mathrm{H}_{2}$ and $\mathrm{D}_{2}$. 
TABLE 37. Specific heats at saturation pressure of normal hydrogen, normal deuterium, and hydrogen deuteride in the solid and liquid states

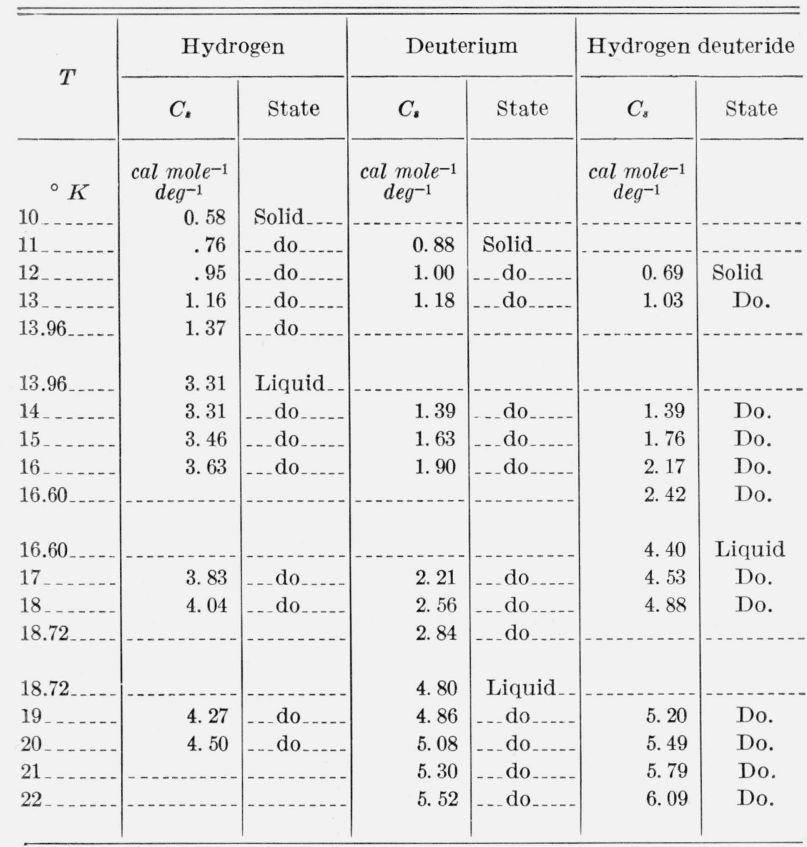

$C_{v}$ along the liquid-vapor line being greater [176].

The difference between $C_{s}$ and $C_{v}$ for hydrogen is large when compared with the differences for other substances having higher boiling temperatures. In general, $\left(C_{s}-C_{v}\right)$ is large for lowboiling substances because of their larger expansivities.

The Debye $\theta$ in the Debye specific heat function that fits the $C_{v}$ data on solid $\mathrm{H}_{2}$ is $105^{\circ} \mathrm{K}$. This may be compared with $91^{\circ} \mathrm{K}$ for $C_{s}$.

The specific heats at constant pressure of compressed liquid hydrogen and gaseous hydrogen were measured by Gutsche [178] for temperatures from $16^{\circ} \mathrm{K}$ to $38^{\circ} \mathrm{K}$ and for pressures of about $10,25,40,60,80$, and $100 \mathrm{~kg} \mathrm{~cm}^{-2}$, using a calorimeter so arranged that approximate constancy of pressure was maintained by manual operation of valves permitting fluid to pass from the calorimeter. As a result of this experimental procedure, the mass of hydrogen in the calorimeter was smaller at the higher temperatures, and consequently the accuracy of measurement is probably lower at the higher temperatures.

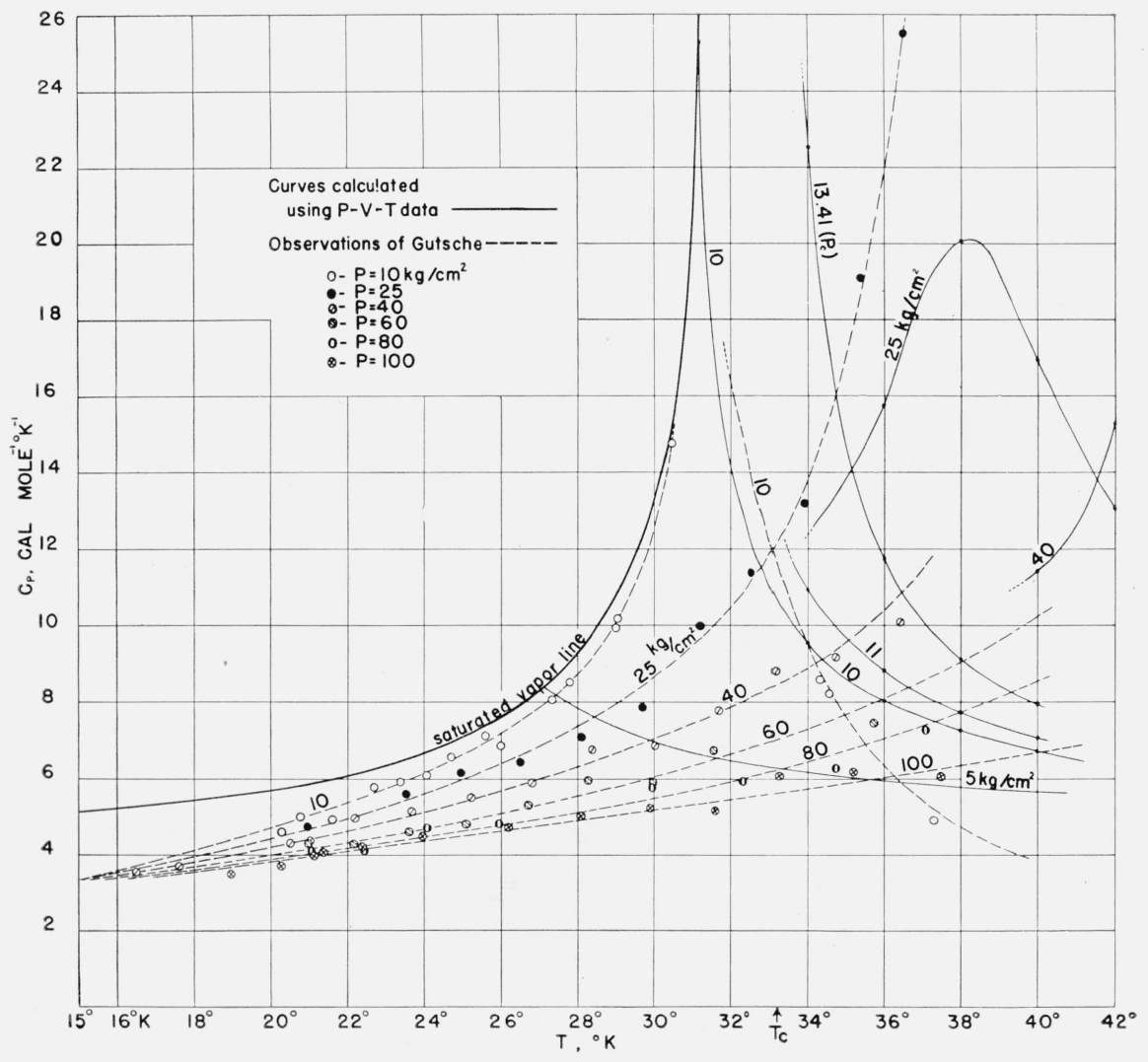

Figure 27. Specific heat, $C_{p}$, of compressed liquid and gaseous $\mathrm{H}_{2}$. 
In figure 27 are plotted Gutsche's experimental data with dotted curves as drawn by Gutsche in his paper to represent the experimental data. The full line curves apply only to the vapor and were obtained by calculation from the PV'T correlations of preceding sections of the paper and specific heats in the ideal gas state, table 8 . The heavy curve shows $C_{p}$ for saturated vapor. The full-line curves beginning on this heavy curve, or saturated vapor line, sloping downward toward the right represent the specific heats, $C_{p}$, for the vapor at pressures of 5 and $10 \mathrm{~kg} \mathrm{~cm} \mathrm{~cm}^{-2}$. Parts of similar curves also based on the PV'T data are shown for 11 and for $13.41 \mathrm{~kg} \mathrm{~cm}^{-2}$, the critical pressure.

For temperatures above the critical, the dashed curves of Gutsche for 10,25 , and $40 \mathrm{~kg} \mathrm{~cm}^{-2}$ are quite different from the full line curves based on PV'T data. The dashed curve for the gas at 10 $\mathrm{kg} \cdot \mathrm{cm}^{-2}$ is certainly incorrect at the highest temperatures, as the actual deviation from the ideal gas law for hydrogen is such as to increase $C_{p}$ above the approximately 5 cal $\mathrm{deg}^{-1}$ mole $^{-1}$ of the ideal gas at these temperatures.

It is seen in figure 27 that Gutsche's experimental values for the liquid scatter considerably. It is believed that Gutsche's recommended values of $C_{p}$ for liquid hydrogen, represented by the dashed lines in figure 27 , are too high. In figure 30 are shown two sets of isobars, $E$ and $E^{\prime}$, on a temperature-entropy diagram for liquid hydrogen. The full-line curves, $E$, were calculated from Gutsche's $C_{p}$ data; the dashed curves, $E^{\prime}$, are the best fit for all the thermal and state data on liquid hydrogen and are the ones used in the construction of the temperature-entropy diagram. As $(d S / d T)_{P}=C_{p} / T$, the two sets of isobars, $E$ and $E^{\prime}$, imply different $C_{p}$ 's and show that Gutsche's values of $C_{p}$ are too high to be consistent with the other data on liquid hydrogen. The differences are of the order of 15 percent in the $C_{p}$ 's of liquid hydrogen. The ratio $C_{p} / C_{v}$ for liquid hydrogen in equilibrium with vapor was calculated from the velocity of sound in liquid hydrogen, and $C_{p}$ was obtained by combining this calculated value of the ratio $\left(C_{p} / C_{v}\right)$, with $C_{v}$ from figure 26 . Pitt and Jackson [175] obtained the value 1,127 m $\mathrm{sec}^{-1}$ for the velocity of sound in liquid hydrogen at $20.46^{\circ} \mathrm{K}$. Using this with a value of $(d V / d P)$ extrapolated from Bartholomé's data (VIII), one

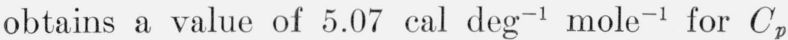

for liquid hydrogen in equilibrium with vapor $(\sim 1 \mathrm{~atm})$ at $20.46^{\circ} \mathrm{K}$.

This is slightly lower than would probably be obtained by extrapolating Gutsche's curves to $1 \mathrm{~atm}$.

\section{(b) $\mathrm{D}_{2}$ and $\mathrm{HD}$}

In figure 24 the specific heats $C_{s}$ at saturation pressure of liquid and solid $n-\mathrm{D}_{2}$ and $\mathrm{HD}$ are compared with $\boldsymbol{C}_{s}$ for $\mathrm{H}_{2}$. The $\mathrm{D}_{2}$ measurements were made by Clusius and Bartholomé [174] and the HD measurements by Brickwedde and Scott [150]. The solid $\mathrm{D}_{2}$ data are fitted, within experimental accuracy, by a Debye function with $\theta=$ $89^{\circ}$. The data on solid HD, however, can not be fitted over the range of measurement with a single value of $\theta$. Thus $\theta$ for $C_{s}$ of $H D$ at $16.3^{\circ} \mathrm{K}$ is $79^{\circ}$, whereas for $12.5^{\circ} \mathrm{K}, \Theta$ is $98^{\circ}$. As the Debye function is intended to represent $C_{v}$, this failure to fit the $C_{s}$ data is not surprising.

In figures 25 and 26 the specific heat $C_{v}$ at constant volume of solid and liquid $\mathrm{D}_{2}$ is compared with $C_{s}$ for $\mathrm{D}_{2}$ and $C_{v}$ for $\mathrm{H}_{2}$. A Debye function with $\theta=97^{\circ}$ fits within experimental accuracy the $C_{v}$ data for solid $\mathrm{D}_{2}$. This value of $\Theta$ for solid $\mathrm{D}_{2}$ may be compared with $105^{\circ}$ for solid $\mathrm{H}_{2}$. According to the simple theory of lattice vibrations, which assumes simple harmonic restoring forces in the lattice, $\theta$ would be proportional to $1 / \sqrt{M}$ and the $\theta$ 's for $H_{2}$ and $D_{2}$ would be in the ratio $\sqrt{4 / 2}=1.41$. The ratio of the experimental values however, is 1.08. This is evidence that the lattice restoring forces in solid $\mathrm{H}_{2}$ and $\mathrm{D}_{2}$ are strongly anharmonic.

\section{Latent Heats of Vaporization}

(a) Normal Hydrogen

Simon and Lange [171] measured the heat of vaporization of normal hydrogen at several temperatures between the triple point and the boiling point. They found that heat of vaporization, in calories per mole, was given by

$$
L_{v}=219.7-0.27(T-16.6)^{2},
$$

where $T$ is the Kelvin temperature.

(b) Mixtures of $o-\mathrm{H}_{2}$ and $p-\mathrm{H}_{2}$

As orthohydrogen and parahydrogen are very closely related, it might be expected that their mixtures would have properties related very simply to those of the pure components. Never- 
theless, the $\mathrm{H}_{2}$ vapor-pressure data of Brickwedde and Scott [146] given by the equations and graphs of Section 7 show that the ortho-para $\mathrm{H}_{2}$ mixtures do not follow Raoult's law for ideal solutions. A simple application of the Clapeyron equation in the form applying to a pure substance indicates that the latent heat of vaporization and the internal energy of the liquid and solid do not follow a linear, but rather an approximately quadratic dependence upon the composition. This same qualitative result is obtained when account is taken of change of composition by fractionation during vaporization. Functions approximately linear in $x$, the ortho mole fraction, are obtained when $L_{\mathrm{mix}}-L_{\mathrm{eq}}$, the difference in latent heats, and $E_{\mathrm{eq}}-E_{\mathrm{m} 1 \mathrm{x}}$, the difference in the internal energy, are divided by $x_{\mathrm{mix}}-x_{\mathrm{eq}}$, the corresponding difference in the ortho mole fraction. The subscript "eq" indicates the ortho-para mixture that is at equilibrium at $20.4^{\circ} \mathrm{K}$, containing 0.21 percent of ortho- and 99.79 percent of parahydrogen. The subscript "mix" refers to any other mixture for which data were obtained. When the line for $\Delta E / \Delta x$ is horizontal, it indicates that ideal solution laws apply. The line has a clear indication of slope, as shown by the continuous lines in figure 28 , indicating that ideal solution laws do not apply. In the graph for $\Delta E / \Delta x$, the points for the liquid include a contribution of about 7 percent related to change of composition due to fractionation. The lower dashed line shows the result when this correction is omitted. For the solid it was thought proper to omit the correction for this effect because departure from equilibrium due to slowness of diffusion in the solid would make it too uncertain. The upper dashed line shows the result for the solid when such a correction for fractionation is included.

The use of straight lines for $\Delta E / \Delta x$, the divided difference of the internal energy, has a theoretical justification apart from the fact that the scattering of individual values is so great as to obscure the exact shape of the curve for the liquid. If the internal energy of the liquid is a simple sum of independent energies of different molecular pairs, all of essentially equal probability of formation, then the energy has the form

$$
E=x^{2} E_{o o}+2 x(1-x) E_{o p}+(1-x)^{2} E_{p p} .
$$

In this case, the differences $E_{\mathrm{eq}}-E_{\mathrm{m} 1 \mathrm{x}}$ divided by the corresponding differences in $x$ for the mix- tures of different compositions will be linearly dependent on $x$. The slope of this line is 2 $E_{o p}-E_{o o}-E_{p p}$ and the value of the ordinate at $x=-x_{\mathrm{eq}}$ is $2\left(E_{p p}-E_{o p}\right)$. From the curves in figure 28, it will thus be found that $E_{p p}-E_{o p}$ is 0.7 cal mole ${ }^{-1}$ and $E_{p p}-E_{o o}$ is 4.2 cal mole ${ }^{-1}$ for the liquid. For the solid the corresponding values are

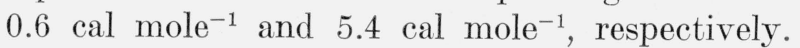
The relative size of $E_{p p}-E_{o o}$ as compared to $E_{p p}-E_{o p}$ suggests that most of the deviation from ideal solution laws is due to special effects between $o-\mathrm{H}_{2}$ molecules.

From the scattering of the points plotted, it appears that ordinates are uncertain to 0.2 or 0.3 cal $\mathrm{mole}^{-1}$ for the liquid and possibly to $1 \mathrm{cal}$ mole $^{-1}$ for the solid. The use of the straight line for $\Delta L / \Delta x$ in figure 29 is very nearly consistent with its use for $\Delta E / \Delta x$ and is allowed within the scattering of the data. Combining the results for the dependence upon composition with the results of Simon and Lange for normal hydrogen, the latent heat of vaporization of liquid hydrogen in calories per mole is approximately

$$
217.0-0.27(T-16.6)^{2}+1.4 x+2.9 x^{2}
$$

for any mixture of $o-\mathrm{H}_{2}$ and $p-\mathrm{H}_{2}$, where $x$ is the orthohydrogen mole fraction.

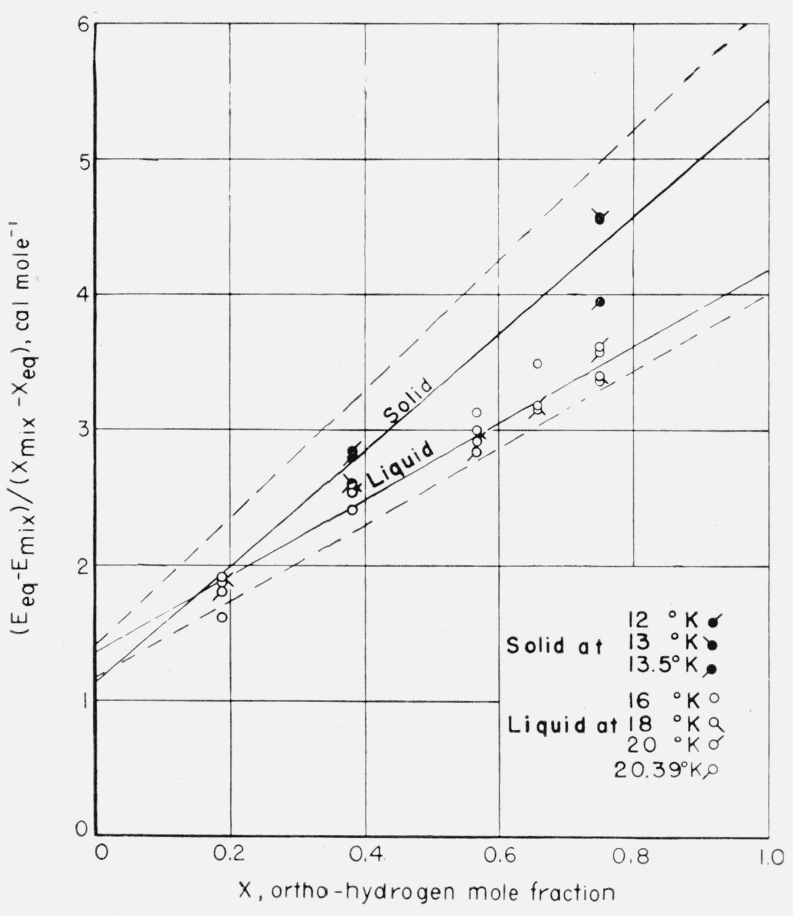

Figure 28. Dependence of internal energy of solid and liquid $\mathrm{H}_{2}$ upon the ortho-para composition. 


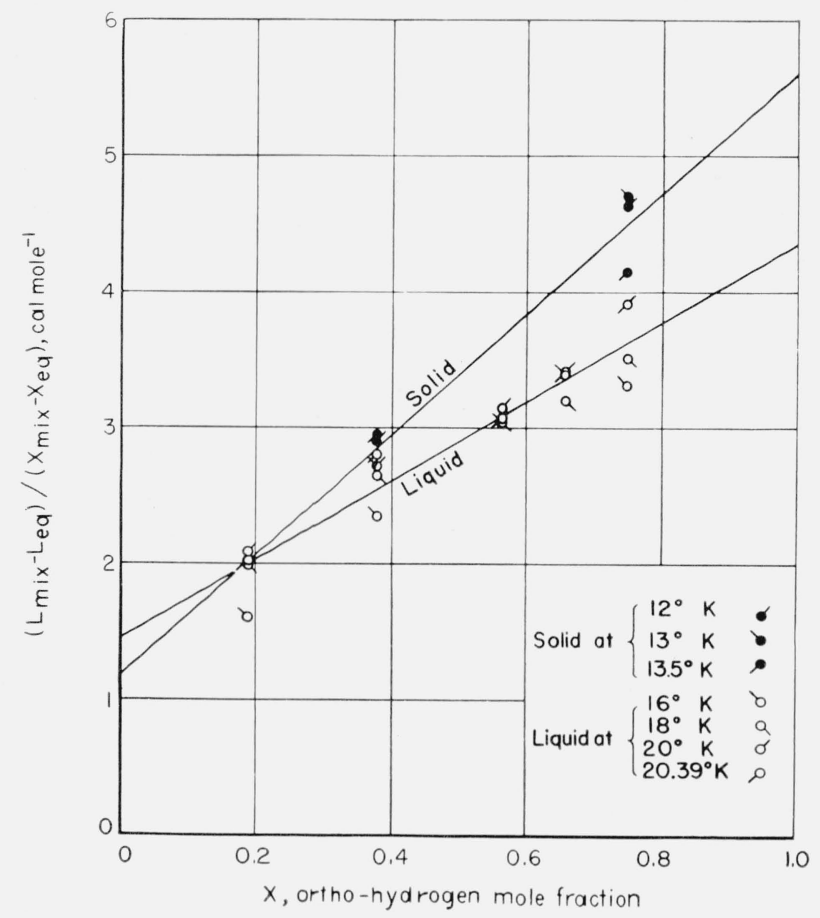

Figure 29. Dependence of latent heats of vaporization and sublimation of hydrogen upon the ortho-para composition.

The heats of fusion of para- and normal hydrogen are reported in table 38 as being equal within 0.03 cal mole $^{-1}$. On the basis of the two distinct straight lines for liquid and solid hydrogen in figure 29 , it would be expected that the difference would be about 0.7 cal mole ${ }^{-1}$. The reason for this discrepancy is not known, though it may suggest that the lines for the liquid and solid should be more nearly identical.

TABLE 38. Latent heats of fusion

\begin{tabular}{|c|c|c|c|}
\hline Substance & $\begin{array}{l}\text { Heat of } \\
\text { fusion }\end{array}$ & $T$ & $P$ \\
\hline Normal hydrogen & $\begin{array}{r}\text { cal mole-1 } \\
28.0\end{array}$ & $\begin{array}{l}{ }^{\circ} K \\
13.95_{7}\end{array}$ & $\begin{array}{r}m m \underset{54.0}{\mathrm{Hg}} \\
54\end{array}$ \\
\hline Parahydrogen & $28.0_{3}$ & 13. $81_{3}$ & 52.8 \\
\hline Normal deuterium & 47.0 & 18. $72_{3}$ & 128.5 \\
\hline Hydrogen deuteride..... & 38.1 & 16. $60_{4}$ & 92.8 \\
\hline
\end{tabular}

The manner in which the vapor pressures depend on composition and temperature has formed the basis for the treatment of latent heats of vaporization given in this section. Cohen and Urey [166] and Schäfer [164] have given theoretical discussions of the vapor pressures of ortho and para $\mathrm{H}_{2}$ and $\mathrm{D}_{2}$. Cohen and Urey did not expect deviations from the law of perfect solutions. Schäfer suggested that forces connected with rotation within the crystal lattice might account for vapor-pressure differences.

\section{(c) Normal Deuterium}

Clusius and Bartholomé [174] measured the heat of vaporization of normal deuterium, obtaining the value 302.3 cal mole ${ }^{-1}$ at $19.70^{\circ} \mathrm{K}$.

(d) Mixtures of $o-D_{2}$ and $p-D_{2}$

The difference in latent heats of vaporization and the approximate difference in internal energies have been calculated from the vapor pressures of the normal and the $20.4^{\circ} \mathrm{K}$ equilibrium mixtures of ortho- and paradeuterium measured by Brickwedde, Scott, and Taylor [149]. PVT data for deuterium as determined by Schäfer were also used in the calculation. As there are data for only two compositions, giving only one difference of composition, it is not possible either to correct for fractionation or to test for deviation from Raoult's Law. It seems improbable that the law holds for deuterium, as it does not hold for hydrogen. The indicated differences in latent heats of vaporization are smaller than for hydrogen. Thus, $L_{\mathrm{norm}}-L_{\mathrm{eq}}=0.3$ cal mole ${ }^{-1}$ for the liquid and $1.0 \mathrm{cal} \mathrm{mole}^{-1}$ for the solid. The same values are obtained for the differences in internal energies, $E_{\text {eq }}-E_{\text {norm. }}$. Cohen and Urey [166] on the basis of their theoretical calculations, concluded that differences in binding energy between corresponding forms should be half as great for $\mathrm{D}_{2}$ as for $\mathrm{H}_{2}$. Considering that the uncertainties in the data for $\mathrm{D}_{2}$ are comparable with the magnitudes themselves, the data can not be said to conflict with the theoretical preduction.

\section{(e) Hydrogen Deuteride}

Brickwedde and Scott [146] measured the heat of vaporization of hydrogen deuteride, obtaining the value $257 \mathrm{cal} \mathrm{mole}^{-1}$ at $22.54^{\circ} \mathrm{K}$.

\section{Latent Heats of Fusion}

The latent heats of fusion of hydrogen, parahydrogen, normal deuterium, and hydrogen deuteride were measured by Simon and Lange [171], Clusius and Hiller [172], Clusius and Bartholomé [174], and by Brickwedde and Scott [150], respec- 
tively, and are listed in table 38 with corresponding vapor pressures and temperatures.

\section{The Temperature-Entropy Diagram}

\section{Data}

Data of several different types were used in determining the temperature-entropy diagram. For the vapor, and for the gas below a density of 500 Amagats, values of the various quantities were obtained by interpolation from tables 14,22 , and 23. The particular difficulties encountered in treating the liquid region will be evident from the

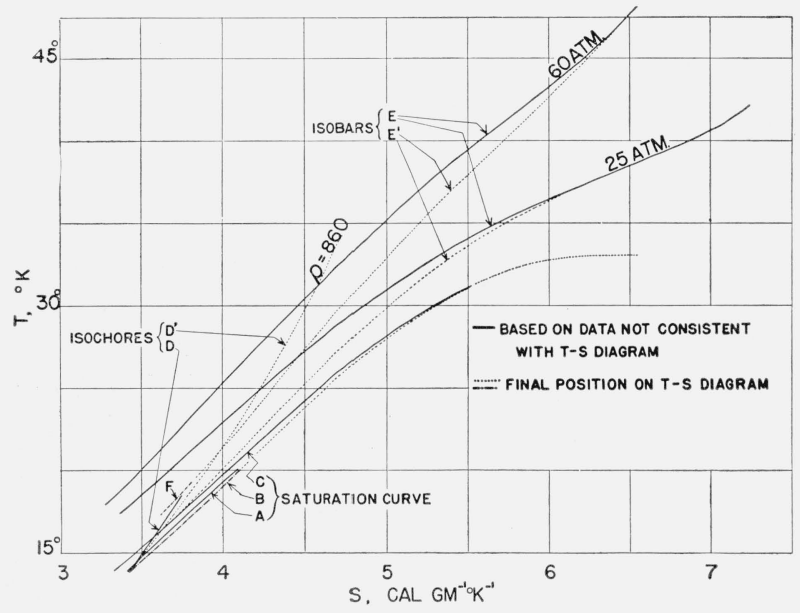

Figure 30. Discrepancies in the thermal data for $\mathrm{H}_{2}$ in the region of the liquid.

following discussion. Discrepancies between the various data for the liquid are shown in figure 30 .

Between the triple point and the boiling point, the entropy of liquid normal hydrogen at saturation pressure was obtained using calorimetric data for the solid and liquid and adding a theoretical value for the entropy of mixing. The result is shown as line $B$ in figure 30 . The entropy of the liquid was also calculated using the theoretical entropy of the ideal gas, correcting to the state of saturated vapor and subtracting the latent heat of vaporization. The latent heat of vaporization was determined in two ways - by direct calorimetric measurement and by using vapor pressures and other data with the Clapeyron equation. Line $A$ is based on calorimetric latent heats and line $C$ on latent heats from vapor pressures. At

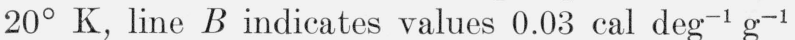
greater than line $A$ and $0.08 \mathrm{cal} \mathrm{deg}^{-1} \mathrm{~g}^{-1}$ greater than line $C$.
Lines of constant density could be obtained for the compressed liquid by integrating $C_{v} / T$, beginning at line $B$. Values of $C_{v}$ from figure 26 were used. The results indicate that these constant density lines are approximately parallel at a given temperature for densities less than 500 Amagats. Data of table 14 indicate that there is a similar parallelism for higher densities near the critical temperature.

Values of entropy of the liquid for various pressures along the $17.34^{\circ} \mathrm{K}$ and $19.28^{\circ} \mathrm{K}$ isotherms were obtained by integration of the equation

$$
(d S / d P)_{T}=-(d V / d T)_{P} .
$$

The values used for $(d V / d T)_{P}$ were based on smoothed values of volume for the liquid as given in table 32 for the temperatures $16.43^{\circ} \mathrm{K}, 18.24^{\circ} \mathrm{K}$, and $20.33^{\circ} \mathrm{K}$. The constant of integration was chosen to fit line $B$. From the results, a set of constant pressure lines, of which the segment $F$ is typical, was obtained for various pressures. In addition, a point that should have been on the 860 Amagat density line was obtained by interpolation and a line $D$ was drawn through it and through the 860 Amagat density point on line $B$ as determined by eq 8.1. The line marked $D^{\prime}$ represents the final correlation.

An unsatisfactory set of values of entropy for the liquid along constant pressure lines was obtained by integrating the $C_{p}$ data of Gutsche, figure 27. Curves $E$ are the results for 25 and 60 atm, while the final correlation gave curves $E^{\prime}$.

\section{Final Correlation}

In the final correlation, the saturation curve $B$ was accepted and the isochores were considered parallel. The isochores at high density were given by integration of $C_{v} / T$, beginning on line $B$. The isochores at intermediate density were obtained by interpolation between values at high density and values below 500 Amagats. The interpolation was made along the $35^{\circ} \mathrm{K}$ isotherm from an entropy-density plot extending from $\rho=860$ Amagats to $\rho=340$ Amagats.

The extension of curve $B$ to temperatures higher than were given by calorimetric data for the liquid was made from the lower parts of the interpolated isochores and the temperature-density relations for the liquid at saturation pressure given by eq 8.1 . 
The constant pressure lines were determined mainly from the vapor-pressure equation and the equation

$$
(d S / d V)_{T}=(d P / d T)_{V}
$$

At lower temperatures the lines were in fair agreement with Bartholome's PVT data, which served to locate them more closely.

The lines of constant enthalpy were determined from integrals of $T d S$ under the constant pressure lines and were checked by integration along the isochores based on the equation

$$
(d H / d T)_{v}=T(d S / d T)_{V}+V(d S / d V)_{T} .
$$

The location of the curves within the dome is quite straightforward, as the fractionation of the ortho-para mixture is too small to affect these curves significantly.

The resulting temperature-entropy diagram for normal hydrogen is presented in composite form in figures 31,32 , and 33 . The thermal units used are based on the calorie, the Kelvin degree, and the gram, with pressures in atmospheres and densities in Amagat units.

The diagram shows lines of constant enthalpy, pressure and density and, in the region of coexistance of liquid and vapor, lines of constant "quality." The painstaking construction of the curves pertaining to the liquid region, amounting to a correlation of the data for the liquid, has been made by Robert N. Schwartz, who has also drawn the remainder of the diagram on the basis of the tables of this paper. 


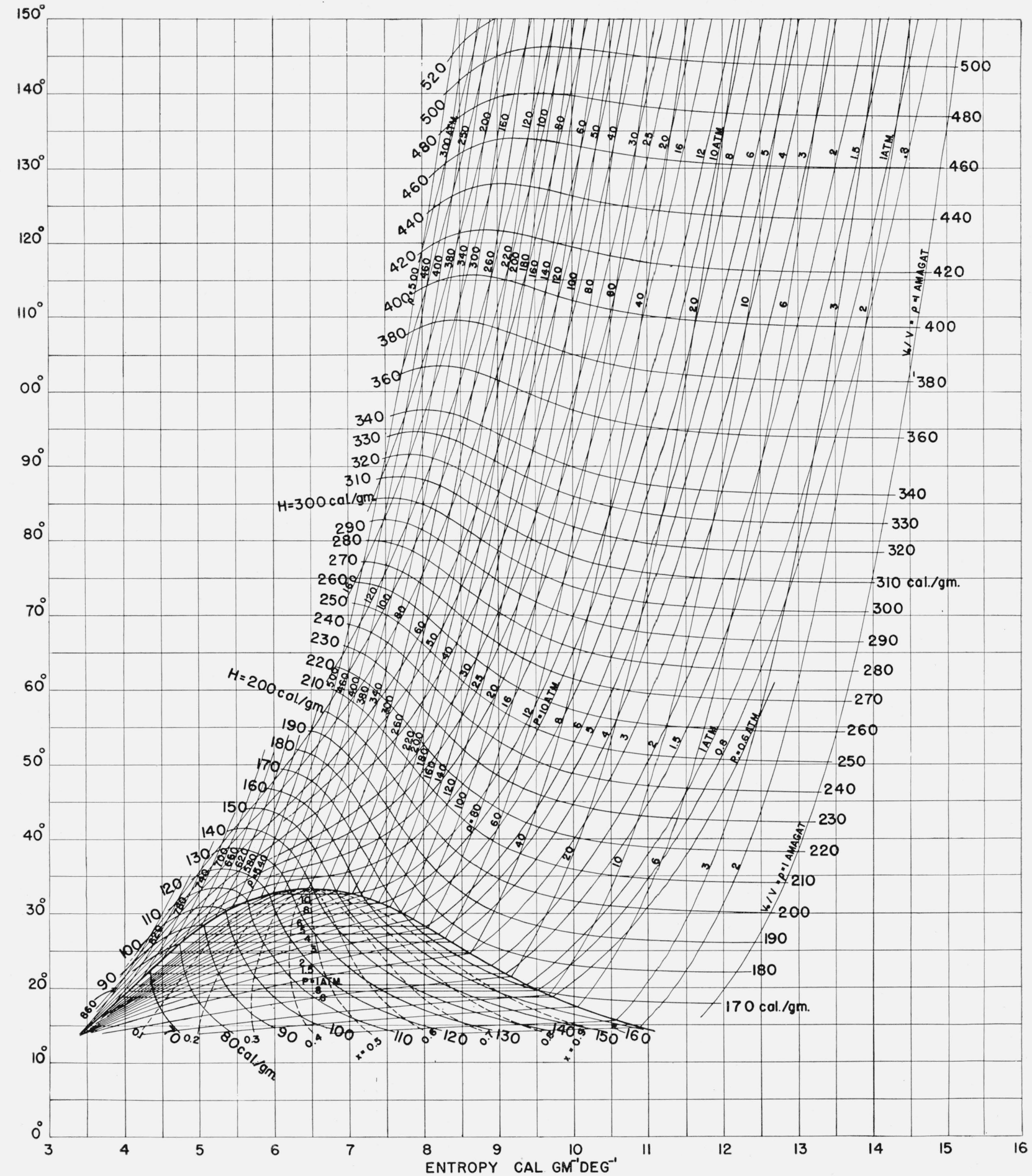

Figure 31. Temperature-entropy diagram for $\mathrm{H}_{2}$ in the region $0^{\circ}$ to $150^{\circ} \mathrm{K}$. 


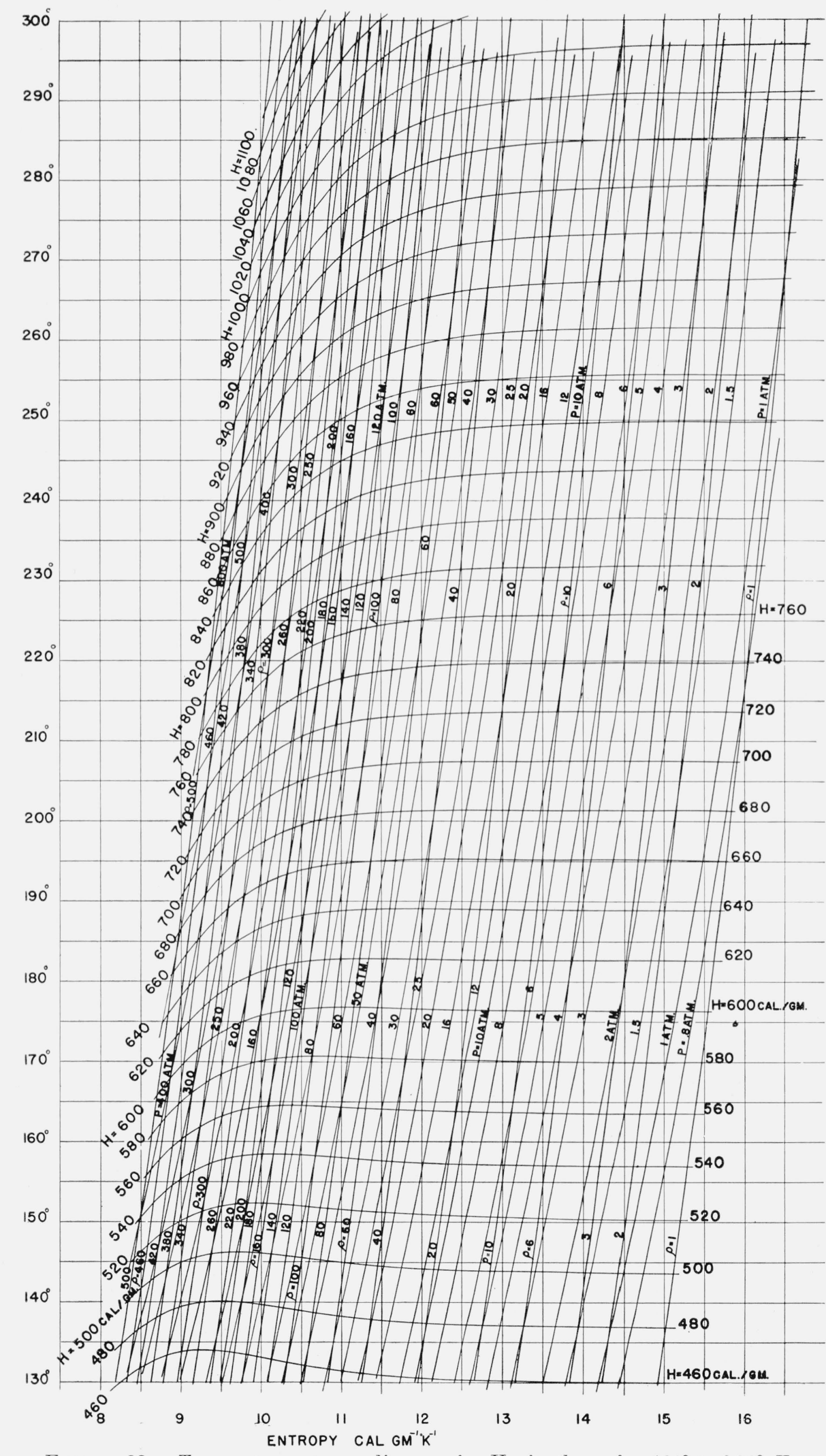

Figure 32. Temperature-entropy diagram for $\mathrm{H}_{2}$ in the region $130^{\circ}$ to $300^{\circ} \mathrm{K}$. 


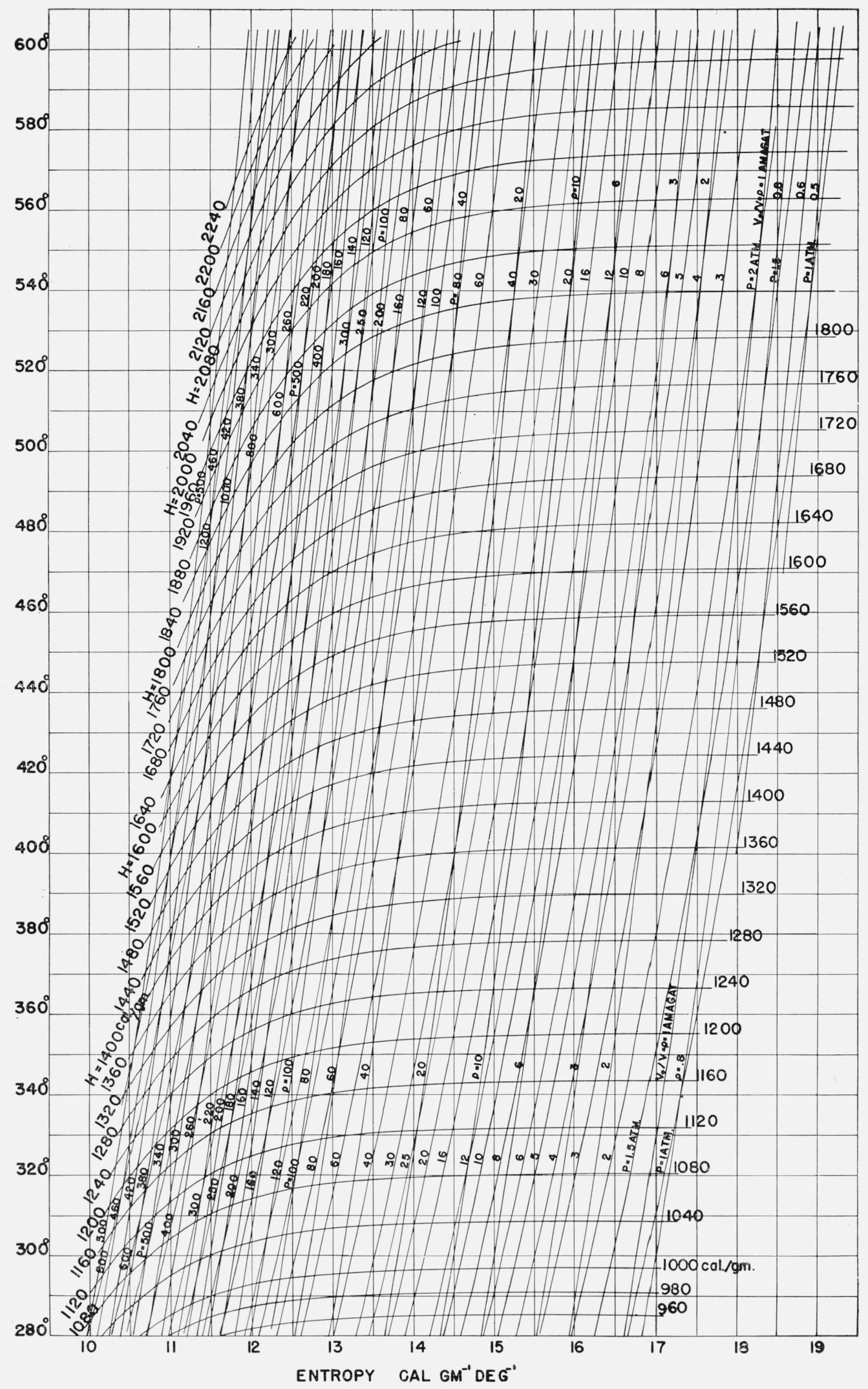

Figure 33. Temperature-entropy diagram for $\mathrm{H}_{2}$ in the region $280^{\circ}$ to $600^{\circ} \mathrm{K}$. 


\section{References}

\section{Thermodynamic Properties of the Hydrogens in the Ideal Gas State}

[1] D. M. Dennison, Proc. Roy. Soc. (London) 115, 483 (1927).

[2] F. Rasetti, Phys. Rev. 34, 367 (1929).

[3] W. F. Giauque, J. Am. Chem. Soc. 52, 4808 (1930).

[4] W. F. Giauque, J. Am. Chem. Soc. 52, 4816 (1930).

[5] H. H. Hyman, Phys. Rev. 36, 187 (1930).

[6] H. H. Hyman and C. R. Jeppesen, Nature 125, 462 (1930).

[7] R. T. Birge and C. R. Jeppesen, Nature 125, 463 (1930).

[8] R. Rydberg, Z. Physik 73, 376 (1931).

[9] O. Klein, Z. Physik $\boldsymbol{7 6 ,} 226$ (1932).

[10] J. L. Dunham, Phys. Rev. 41, 721 (1932).

[11] R. W. Harkness and W. E. Deming, J. Am. Chem. Soc. 54, 2850 (1932).

[12] C. R. Jeppesen, Phys. Rev. 44, 165 (1933).

[13] H. C. Urey and D. Rittenberg, J. Chem. Phys. 1, 137 (1933).

[14] G. N. Lewis and M. F. Ashley, Phys. Rev. 43, 837 (1933).

[15] C. R. Jeppesen, Phys. Rev. 45, 480 (1934).

[16] K. Mie, Z. Physik 91, 475 (1934).

[17] C. O. Davis and H. L. Johnston, J. Am. Chem. Soc. 56, 1045 (1934).

[18] H. L. Johnston and E. A. Long, J. Chem. Phys. 2, 389 (1934)

[19] R. Wildt, Z. Astrophysik 9, 176 (1934).

[20] H. Beutler, Z. physik. Chem. [B] 27, 287 (1934).

[21] H. Beutler, Z. physik. Chem. [B] 29, 315 (1935).

[22] G. K. Teal and G. E. MacWood, J. Chem. Phys. 3, 760 (1935)

[23] Y. Fujioka and T. Wada, Sci. Papers Inst. Phys. Chem. Research (Komagome, Hongo, Tokyo) $2 \boldsymbol{\gamma}$, 210 (1935)

[24] C. R. Jeppesen, Phys. Rev. 49, 797 (1936).

[25] I. Sandeman, Proc. Roy. Soc. Edinburgh 59, 130 (1938-39).

[26] R. T. Birge, Rev. Modern Phys. 13, 233 (1941).

[27] H. W. Woolley, J. Chem. Phys. 9, 470 (1941).

[28] D. D. Wagman, J. E. Kilpatrick, W. J. Taylor, K. S. Pitzer, and F. D. Rossini, J. Research NBS 34, 143 (1945) RP1634.

[29] G. N. Lewis and M. Randall, Thermodynamics and the free energy of chemical substances (McGrawHill Book Co., Inc., New York, N. Y., 1923).

[30] P. S. Epstein, Textbook on thermodynamics (John Wiley and Sons, Inc., New York, N. Y., 1937).

[31] F. H. Mac Dougall, Thermodynamics and chemistry (John Wiley and Sons, Inc., New York, N. Y., 1939).

[32] S. Glasstone, Textbook of physical chemistry (D. Van Nostrand Co., Inc., New York, N. Y., 1940).

\section{Thermal Measurements on Gaseous Hydrogen}

[33] O. Lummer and E. Pringsheim, Wied. Ann. 64, 555 (1898).
[34] M. Pier, Z. Elektrochem. 16, 897 (1910).

[35] N. Bjerrum, Z. Elektrochem. 18, 101 (1912).

[36] A. Eucken, Sitzber. preuss. Akad. Wiss. 1912, 14] (1912).

[37] K. Scheel and W. Heuse, Ann. Physik 40, 473 (1913).

[38] I. Langmuir and G. M. J. Mackay, J. Am. Chem. Soc. 36, 1708 (1914).

[39] I. Langmuir, J. Am. Chem. Soc. 37, 417 (1915).

[40] J. M. Crofts, J. Chem. Soc. 107, 290 (1915).

[41] M. C. Shields, Phys. Rev. 10, 525 (1917).

[42] M. Trautz and K. Hebbel, Ann. Physik 74, 285 (1924).

[43] J. H. Brinkworth, Proc. Roy. Soc. (London) 107, 510 (1925).

[44] J. H. Partington and A. B. Howe, Proc. Roy. Soc. (London) 109, 286 (1925).

[45] F. A. Giacomini, Phil. Mag. 50, 146 (1925).

[46] R. E. Cornish and E. D. Eastman, J. Am. Chem. Soc. 50, 627 (1928).

[47] F. R. Bichowsky and L. C. Copeland, J. Am. Chem. Soc. 50, 1315 (1928).

[48] A. Eucken and K. Hiller, Z. physik. Chem. [B] 4, 142 (1929).

[49] E. J. Workman, Phys. Rev. 3\%, 1345 (1931).

[50] A. Eucken and O. Mücke, Z. physik. Chem. 18, 167 (1932).

[51] K. Wohl and M. Magat, Z. physik. Chem. 19, 117 (1932).

[52] A. Farkas, L. Farkas, and P. Harteck, Proc. Roy. Soc. [A] 144, 481 (1934).

[53] W. T. David and A. S. Leah, Phil. Mag. 18, 307 (1934).

[54] D. Rittenberg, W. Bleakney, H. C. Urey, J. Chem. Phys. 2, 48 (1934).

[55] A. J. Gould, W. Bleakney, and H. S. Taylor, J. Chem. Phys. 2, 362 (1934).

[56] R. W. Fenning and A. C. Whiffen, Phil. Trans. Roy. Soc. (London), 238, 149 (1939).

[57] H. L. Johnston, I. I. Bezman, and C. B. Hood, J. Am. Chem. Soc. 68, 2367 (1946).

[58] H. L. Johnston, C. A. Swanson, and H. E. Wirth, J. Am. Chem. Soc. 68, 2373 (1946).

\section{PVT Relations for Gaseous Hydrogens}

[59] H. Kamerlingh Onnes and C. Braak, Commun. Phys. Lab. Univ. Leiden 97a (1906), 99a, 100a (1907).

[60] H. Kamerlingh Onnes and W. J. de Haas, Commun. Phys. Lab. Univ. Leiden 127e (1912).

[61] P. Kohnstamm and K. W. Walstra, Koninkl. Akad. Wetenschappen Amsterdam, Proc. 17, 203 (1914).

[62] H. Kamerlingh Onnes, C. A. Crommelin, and P. G. Cath, Commun. Phys. Lab. Univ. Leiden 151c (1917).

[63] L. Holborn, Ann. Physik 63, 674 (1920).

[64] J. Palacios Martinez and H. Kamerlingh Onnes, Commun. Phys. Lab. Univ. Leiden 164 (1923).

[65] H. Kamerlingh Onnes and F. M. Penning, Commun. Phys. Lab. Univ. Leiden 165b (1923).

[66] C. A. Crommelin and J. C. Swallow, Commun. Phys. Lab. Univ. Leiden 17\%a (1924).

[67] L. Holborn and J. Otto, Z. Physik 23, 77 (1924). 
[68] F. P. G. A. J. van Agt and H. Kamerlingh Onnes Commun. Phys. Lab. Univ. Leiden 176b (1925).

[69] F. P. G. A. J. van Agt, Commun. Phys. Lab. Univ. Leiden 176c (1925).

[70] L. Holburn and J. Otto, Z. Physik 33, 1 (1925).

[71] L. Holburn and J. Otto, Z. Physik 38, 359 (1926).

[72] G. P. Nijhoff and W. H. Keesom, Commun. Phys. Lab. Univ. Leiden 188d (1927).

[73] E. P. Bartlett, J. Am. Chem. Soc. 49, 687 (1927).

[74] E. P. Bartlett, H. L. Cupples, and T. H. Tremearne, J. Am. Chem. Soc. 50, 1275 (1928).

[75] G. P. Nijhoff and W. H. Keesom, Commun. Phys. Lab. Univ. Leiden 188e (1928).

[76] E. P. Bartlett, H. C. Hetherington, H. M. Kvalnes, and T. H. Tremearne, J. Am. Chem. Soc. 5\%, 1363 (1930).

[77] A. van Itterbeek and W. H. Keesom, Commun. Phys. Lab. Univ. Leiden, 216c (1931).

[78] A. van Itterbeek, Commun. Phys. Lab. Univ. Leiden, Supp. \%ob (1931).

[79] A. Michels, G. P. Nijhoff, and A. J. J. Gerver, Ann. Physik 12, 562 (1932).

[80] W. E. Deming and L. E. Shupe, Phys. Rev. 40, 848 (1932).

[81] A. Michels and A. J. J. Gerver, Ann. Physik 16, 745 (1933).

[82] W. E. Deming and L. S. Deming, Phys. Rev. 45, 109 (1934).

[83] J. B. M. Coppock, Trans. Faraday Soc. 31, 913 (1935).

[84] G. E. Uhlenbeck and E. Beth, Physica 3, 729 (1936).

[85] K. Schäfer, Z. physik. Chem. [B] 36, 85 (1937).

[86] K. Schäfer, Z. physik. Chem. [B] 38, 187 (1937).

[87] J. de Boer and A. Michels, Physica 5, 945 (1938).

[88] R. Wiebe and V. L. Gaddy, J. Am. Chem. Soc. 60, 2300 (1938).

[89] F. G. Keyes, Gas thermometer scale corrections based on an objective correlation of available data for hydrogen, helium, and nitrogen; from: Temperature, its measurement and control in science and industry, American Institute of Physics, (Reinhold Publishing Corporation, 1941).

[90] C. S. Cragoe, Slopes of the PV isotherms of some thermodynamic gases at pressures below two atmospheres; from: Temperature, its measurement and control in science and industry, American Institute of Physics (Reinhold Publishing Corporation, 1941); J. Research NBS 26, 495 (1941) RP1393.

[91] A. Michels and M. Goudeket, Physica 8, 347 (1941).

[92] A. Michels and M. Goudeket, Physica 8, 353 (1941).

[93] A. Michels and M. Goudeket, Physica 8, 387 (1941).

\section{Viscosity of Gaseous Hydrogen}

[94] M. Trautz and P. B. Baumann, Ann. Physik 2, 733 (1929).

[95] M. Trautz and F. W. Stauf, Ann. Physik 2, 737 (1929).

[96] M. Trautz and W. Ludewigs, Ann. Physik 3, 409 (1929).

[97] M. Trautz and H. E. Binkele, Ann. Physik 5, 561 (1930).
[98] M. Trautz and A. Melster, Ann. Physik y, 409 (1930). [99] M. Trautz and R. Zink, Ann. Physik y, 427 (1930). [100] M. Trautz and F. Kurz, Ann. Physik 9, 981 (1931). [101] M. Trautz and K. G. Sorg, Ann. Physik 10, 81 (1931).

[102] M. Trautz and R. Heberling, Ann. Physik 20, 118 (1934).

[103] B. P. Sutherland and O. Maass, Canadian J. Research 6, 428 (1932).

[104] H. Adzumi, Bul. Chem. Soc. (Japan) 12, 199 (1937).

[105] A. van Itterbeek and Miss A. Claes, Nature 142, 793 (1938) and Physica 5, 938 (1938).

[106] A. van Itterbeek and O. van Paemal, Physica 7, 265 (1940).

[107] A. van Itterbeek and O. van Paemal, Physica 7,273 (1940).

[108] W. H. Keesom and P. H. Keesom, Physica 7, 29 (1940).

[109] H. L. Johnston and K. E. MeCloskey, J. Phys. Chem. 44, 1038 (1940).

[110] R. Wobser and F. Müller, Kolloid-Beihefte 52, 165 (1941).

\section{Thermal Conductivity of Gaseous Hydrogen}

[111] A. Schleiermacher, Wied. Ann. 36, 346 (1889).

[112] A. Winkelmann, Wied. Ann. 44, 177 and 429 (1891).

[113] P. A. Eckerlein, Ann. Physik 3, 120 (1900).

[114] P. Gunther, Dissertation, Halle (1906).

[115] A. Eucken, Physik. Z. 12, 1101 (1911).

[116] A. Eucken, Physik Z. 14, 324 (1913).

[117] S. Weber, Ann. Physik 54, 437 (1917).

[118] E. Schneider, Ann. Physik 79, 177 (1926).

[119] E. Schneider, Ann. Physik 80, 215 (1926).

[120] H. Gregory and C. T. Archer, Proc. Roy. Soc. [A] 110, 91 (1926)

[121] K. F. Bonhoeffer and P. Harteck, Z. physik. Chem. [B] 4, 113 (1929).

[122] P. Harteck and H. W. Schmidt, Z. physik. Chem. [B] 21, 447 (1933).

[123] B. G. Dickens, Proc. Roy. Soc. (London) [A] 143, 517 (1934)

[124] W. G. Kannuluik and L. H. Martin, Proc. Roy. Soc. (London) [A] 144, 496 (1934).

[125] H. S. Gregory, Proc. Roy. Soc. (London) [A] 149, 35 (1935).

[126] W. Northdurft, Ann. Physik 28, 137 (1937).

[127] C. T. Archer, Proc. Roy. Soc. 165, 474 (1938).

[128] H. Spencer-Gregory and E. H. Dock, Phil. Mag. 25, 129 (1938).

[129] N. B. Vargaftik and I. D. Parfenov, J. Exptl. Theoret. Phys. (U. S. S. R.) 8, 189 (1938).

[130] W. G. Kannuluik, Proc. Roy. Soc. (London) [A] 175, 36 (1940).

[131] H. L. Johnston and E. R. Grilly, J. Chem. Phys. 14, 233 (1946).

\section{Viscosity and Thermal Conductivity of Gaseous Hydrogen at High Pressure}

[132] D. Enskog, Kungl. Svenska Vetenskaps Akademiens Handl. 63, No. 4 (1921).

[133] H. B. Phillips, J. Math. Phys. 1, 42 (1922). 
[134] J. H. Boyd, Jr., Phys. Rev. 35, 1284 (1930).

[135] R. O. Gibson, Dissertation, Amsterdam (1933).

[136] E. W. Comings and R. S. Egly, Ind. Eng. Chem. 32, 714 (1940).

137] S. Chapman and T. G. Cowling, The mathematical theory of nonuniform gases, (Cambridge at the University Press, 1939).

\section{Viscosity of Liquid Hydrogen}

[138] J. E. Verschaffelt and C. Nicaise, Commun. Phys. Lab. Leiden 151g (1917).

[139] W. H. Keesom and G. E. MacWood, Physica 5, 745 (1938)

[140] H. E. Johns, Can. J. Research 17 [A] 221 (1939).

\section{Vapor Pressures}

[141] H. Kamerlingh Onnes and W. H. Keesom, Commun. Phys. Lab. Univ. Leiden, 137d (1913).

[142] P. G. Cath and H. Kamerlingh Onnes, Commun. Phys. Lab. Univ. Leiden, 152a (1917).

[143] J. Palacios Martinez and H. Kamerlingh Onnes, Commun. Phys. Lab. Univ. Leiden, 156b (1922).

[144] F. Henning, Z. Physik 40, 775 (1926).

[145] W. H. Keesom, A. Bijl, and Miss H. Van der Horst, Commun. Phys. Lab. Univ. Leiden, 217a (1931).

[146] F. G. Brickwedde and R. B. Scott, The vapor pressures of mixtures of ortho and para hydrogen (Unpublished).

[147] E. Cremer and M. Polanyi, Z. physik. Chem. [B] 21, 459 (1933).

[148] R. B. Scott, F. G. Brickwedde, H. C. Urey, and M. H. Wahl, J. Chem. Phys. 2, 454 (1934).

[149] F. G. Brickwedde, R. B. Scott, and H. S. Taylor, J. Research NBS 15, 463 (1935) RP841; J. Chem. Phys. 3, 653 (1935).

[150] F. G. Brickwedde and R. B. Scott, Vapor pressures, specific heats, heats of transition and molecular volumes of liquid and solid hydrogen deuteride (Unpublished).

[151] R. D. O'Neal and M. Goldhaber, Phys. Rev. 58, 574 (1940)

[152] W. F. Libby and C. A. Barter, J. Chem. Phys. 10, 184 (1942).

\section{Melting Curves}

[153] H. Kamerlingh Onnes and W. van Gulik, Commun. Phys. Lab. Univ. Leiden 184a (1926).

[154] W. van Gulik and W. H. Keesom, Commun. Phys. Lab. Univ. Leiden 192b (1928),

[155] F. Simon, M. Ruhemann, and W. A. M. Edwards, Z. physik. Chem. [B] 6, 331 (1929).

[156] W. H. Keesom and J. H. C. Lisman, Commun. Phys. Lab. Univ. Leiden 213e (1931).
[157] W. H. Keesom and J. H. C. Lisman, Commun. Phys. Lab. Univ. Leiden R21a (1932).

\section{PVT Relations for Condensed Phases}

[158] J. Dewar, Proc. Roy. Soc. [A] 73, 251 (1904).

[159] H. Kamerlingh Onnes and C. A. Crommelin, Commun. Phys. Lab. Univ. Leiden 137a (1913).

[160] H. Augustin, Ann. Physik 46, 419 (1915).

[161] E. Mathias, C. A. Crommelin, and H. Kamerlingh Onnes, Commun. Phys. Lab. Univ. Leiden 154b (1921).

[162] W. H. Keesom, J. de Smedt, and H. H. Mooy, K. Akad. Wetensch. Amsterdam, Proc. 33, 8, 814 (1930); Commun. Phys. Lab. Univ. Leiden 209d (1930).

[163] R. B. Scott and F. G. Brickwedde, J. Research NBS 19, 237 (1937) RP1023.

[164] K. Schäfer, Naturwissenschaften $\mathbf{2 6 ,} 563$ (1938).

[165] Helen D. Megaw, Phil. Mag. 28, 129 (1939).

[166] K. Cohen and H. C. Urey, J. Chem. Phys. 7, 157 (1939).

\section{Thermal Properties of Condensed Hydrogen}

[167] W. H. Keesom, Commun. Phys. Lab. Univ. Leiden 137e (1911).

[168] A. Eucken, Verh. deut. Phys. Ges. 18, 18 (1916).

[169] A. Eucken, Verh. deut. Phys. Ges. 18, 4 (1916).

[170] W. H. Keesom and H. Kamerlingh Onnes, Commun. Phys. Lab. Univ. Leiden $\mathbf{1 5 3} a$ (1917).

[171] F. Simon and F. Lange, Z. Physik 15, 312 (1923).

[172] K. Clusius and K. Hiller, Z. physik. Chem. [B] 4, 158 (1929).

[173] K. Mendelssohn, M. Ruhemann, and F. Simon, Z. physik. Chem. [B] 15, 121 (1931).

[174] K. Clusius and E. Bartholomé, Z. physik. Chem. [B] 30, 237 (1935).

[175] A. Pitt and W. J. Jackson, Can. J. Research 1\%, 686 (1935).

[176] E. Bartholomé and A. Eucken, Z. Elektrochem. 42, 547 (1936)

[177] E. Bartholomé, Z. physik. Chem. [B] 33, 387 (1936).

[178] H. Gutsche, Z. physik. Chem. [A] 184, 45 (1939).

\section{Unclassified}

[179] G. Rutledge, Phys. Rev. 40, 262 (1932).

[180] H. J. Hoge and F. G. Brickwedde, J. Research NBS 22, 351 (1939) RP1188.

[181] Mathematical Tables Project, Tables of Lagrangian Interpolation Coefficients, (Columbia University Press, 1944).

Washington, August 7, 1947. 